Florida International University FIU Digital Commons

3-24-2011

\title{
Hybrid Approaches to Estimating Freeway Travel Times Using Point Traffic Detector Data
}

Yan Xiao

Florida International University, yxiao001@fiu.edu

DOI: $10.25148 /$ etd.FI1 1042503

Follow this and additional works at: https://digitalcommons.fiu.edu/etd

\section{Recommended Citation}

Xiao, Yan, "Hybrid Approaches to Estimating Freeway Travel Times Using Point Traffic Detector Data" (2011). FIU Electronic Theses and Dissertations. 356.

https://digitalcommons.fiu.edu/etd/356

This work is brought to you for free and open access by the University Graduate School at FIU Digital Commons. It has been accepted for inclusion in FIU Electronic Theses and Dissertations by an authorized administrator of FIU Digital Commons. For more information, please contact dcc@fiu.edu. 


\section{FLORIDA INTERNATIONAL UNIVERSITY}

Miami, Florida

\section{HYBRID APPROACHES TO ESTIMATING FREEWAY TRAVEL TIMES USING POINT TRAFFIC DETECTOR DATA}

A dissertation submitted in partial fulfillment of the

requirements for the degree of DOCTOR OF PHILOSOPHY

in

CIVIL ENGINEERING

by

Yan Xiao

2011 


\section{To: Dean Amir Mirmiran}

College of Engineering and Computing

This dissertation, written by Yan Xiao, and entitled Hybrid Approaches to Estimating Freeway Travel Times Using Point Traffic Detector Data, having been approved in respect to style and intellectual content, is referred to you for judgment.

We have read this dissertation and recommend that it be approved.

$\begin{array}{r}\hline \text { Albert Gan } \\ \hline \text { L. David Shen } \\ \hline \text { Zhenmin Chen } \\ \hline \text { Mohammed Hadi, Major Professor }\end{array}$

Date of Defense: March 24, 2011

The dissertation of Yan Xiao is approved.

\begin{tabular}{r} 
Dean Amir Mirmiran \\
College of Engineering and Computing \\
\hline Interim Dean Kevin O'Shea \\
University Graduate School
\end{tabular}

Florida International University, 2011 


\section{DEDICATION}

I dedicate this dissertation to my family for their love and support. 


\section{ACKNOWLEDGMENTS}

My sincere gratitude and appreciation goes first to my major advisor, Dr. Mohammed Hadi, for his continued guidance, encouragement, inspiration, and support during my doctoral study at Florida International University. His intelligence and diligence has inspired me and I am grateful for his mentorship. What I have learned from Dr. Hadi will be invaluable to my future career and the rest of my life.

I am also grateful to the members of my dissertation committee, Dr. Albert Gan, Dr. David Shen, and Dr. Zhenmin Chen for serving on my committee, and for their comments, suggestions, and interest in my research. Special thanks go to Dr. Zhao, who devoted her whole life to teaching and research, and has set a respectable example for me.

I would like to extend my deep appreciation to Dr. Halit Ozen for his invaluable advice. I would also like to thank Dr. Chengjun Zhan and Patricio Alvarez for their interesting technical discussions.

Furthermore, I would like to acknowledge financial support for this research from the Florida Department of Transportation Research Center and also from the FIU Graduate School Dissertation Year Fellowship. I am also grateful to Mr. Javier Rodriguez, Mr. Manuel Fontan, and Mr. Joseph Snyder of the Florida Department of Transportation District 6 SunGuide Traffic Management Center for their assistance in collecting the travel time data used in this research.

Finally, I would like to thank my family for their consistent encouragement and

love. Without their patience, understanding, and support, the completion of this work would not have been possible. 


\title{
ABSTRACT OF THE DISSERTATION \\ HYBRID APPROACHES TO ESTIMATING FREEWAY TRAVEL TIMES USING \\ POINT TRAFFIC DETECTOR DATA
}

\author{
by
}

Yan Xiao

Florida International University, 2011

Miami, Florida

\section{Professor Mohammed Hadi, Major Professor}

The accurate and reliable estimation of travel time based on point detector data is needed to support Intelligent Transportation System (ITS) applications. It has been found that the quality of travel time estimation is a function of the method used in the estimation and varies for different traffic conditions. In this study, two hybrid on-line travel time estimation models, and their corresponding off-line methods, were developed to achieve better estimation performance under various traffic conditions, including recurrent congestion and incidents. The first model combines the Mid-Point method, which is a speed-based method, with a traffic flow-based method. The second model integrates two speed-based methods: the Mid-Point method and the Minimum Speed method. In both models, the switch between travel time estimation methods is based on the congestion level and queue status automatically identified by clustering analysis. During incident conditions with rapidly changing queue lengths, shock wave analysis-based refinements are applied for on-line estimation to capture the fast queue propagation and recovery. 
Travel time estimates obtained from existing speed-based methods, traffic flow-based methods, and the models developed were tested using both simulation and real-world data. The results indicate that all tested methods performed at an acceptable level during periods of low congestion. However, their performances vary with an increase in congestion. Comparisons with other estimation methods also show that the developed hybrid models perform well in all cases. Further comparisons between the on-line and off-line travel time estimation methods reveal that off-line methods perform significantly better only during fast-changing congested conditions, such as during incidents.

The impacts of major influential factors on the performance of travel time estimation, including data preprocessing procedures, detector errors, detector spacing, frequency of travel time updates to traveler information devices, travel time link length, and posted travel time range, were investigated in this study. The results show that these factors have more significant impacts on the estimation accuracy and reliability under congested conditions than during uncongested conditions. For the incident conditions, the estimation quality improves with the use of a short rolling period for data smoothing, more accurate detector data, and frequent travel time updates. 


\section{TABLE OF CONTENTS}

CHAPTER

PAGE

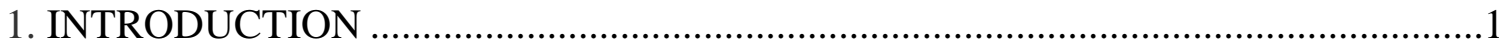

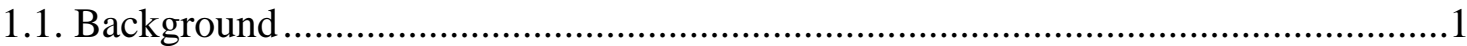

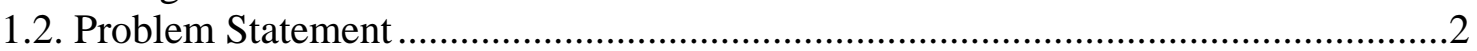

1.3. Research Goal and Objectives .....................................................................4

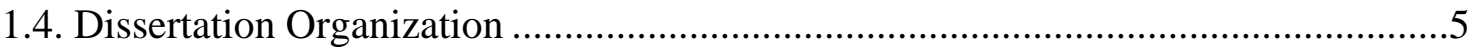

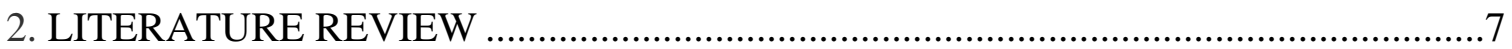

2.1. Travel Time Data Collection Techniques ............................................................... 7

2.1.1. Direct Measurement Techniques for Travel Time....................................... 7

2.1.2. Indirect Measurement Techniques for Travel Time ......................................9

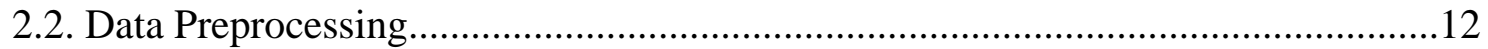

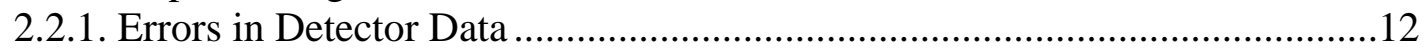

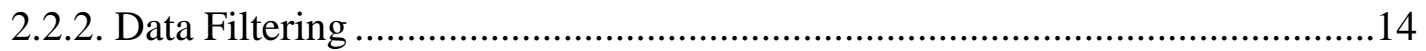

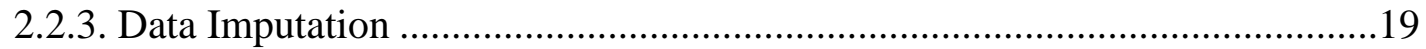

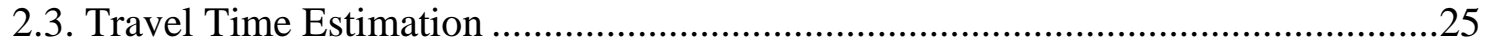

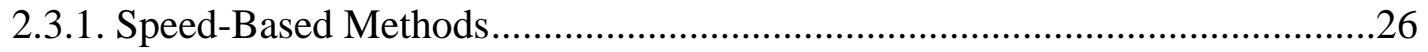

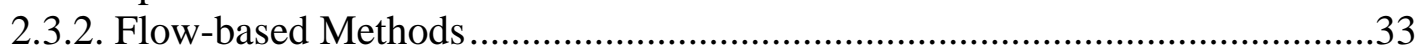

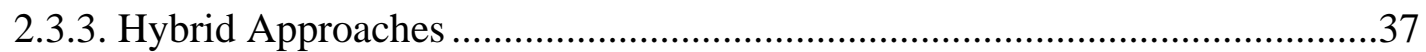

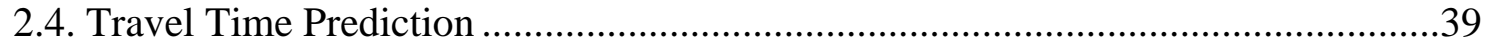

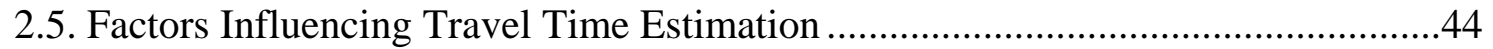

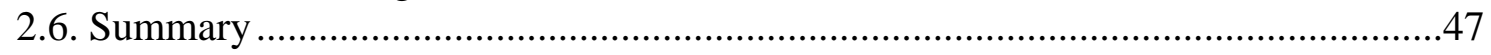

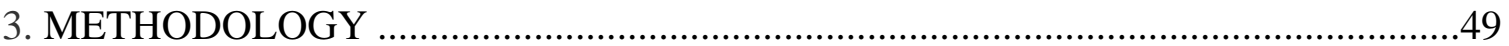

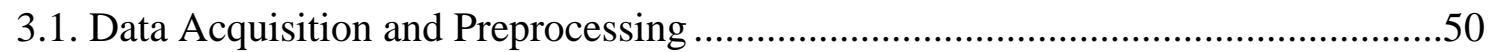

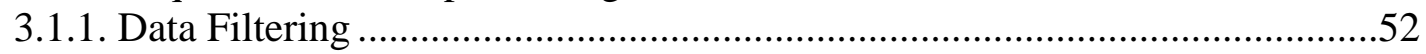

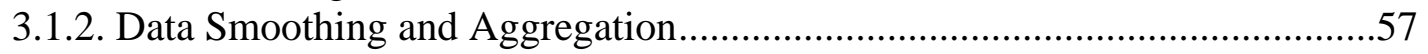

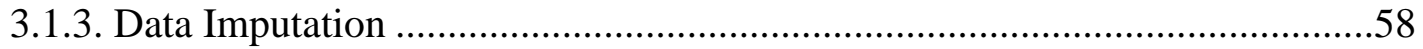

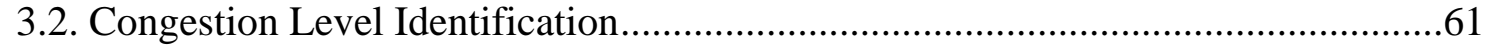

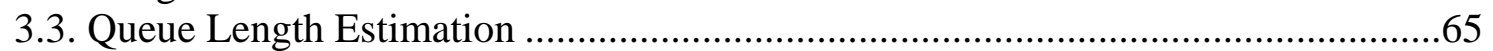

3.4. On-Line Travel Time Estimation....................................................................68

3.4.1. On-Line Hybrid Model 1 ...........................................................................68

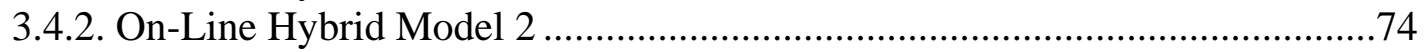

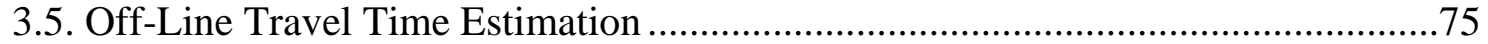

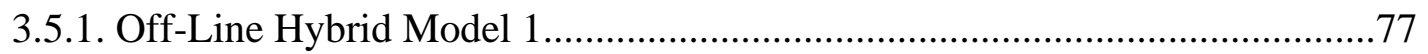

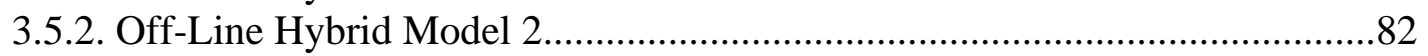

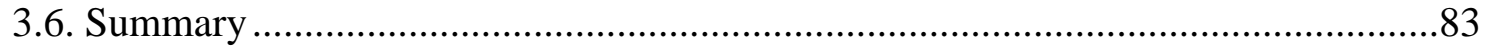

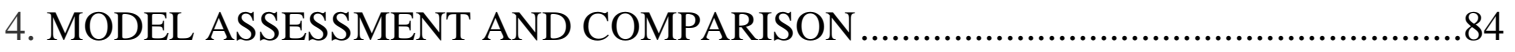

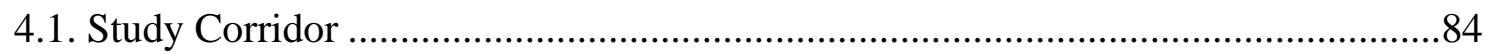

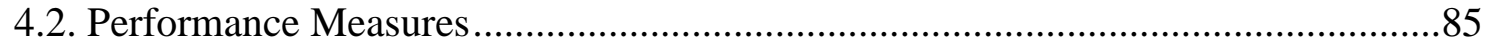

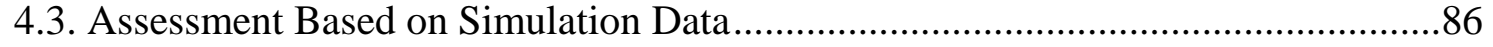

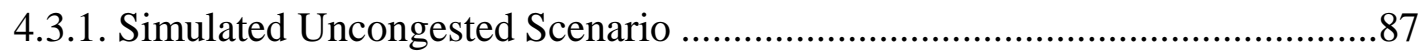

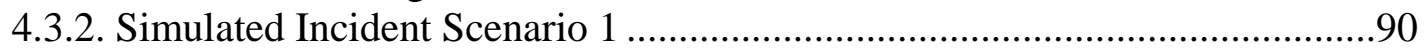


4.3.3. Simulated Incident Scenario 2 ..................................................................97

4.4. Comparison Based on Real-world Data...............................................................102

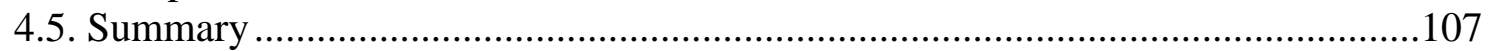

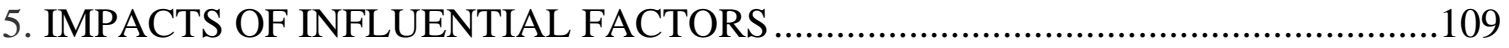

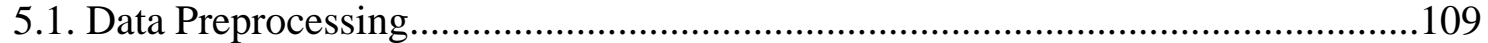

5.1.1. Data Smoothing ………………………................................................109

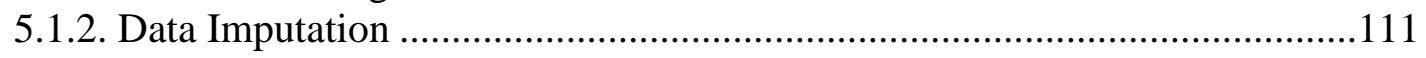

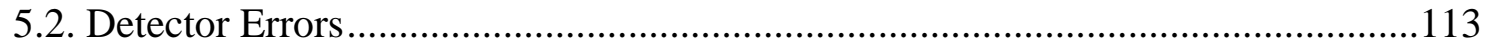

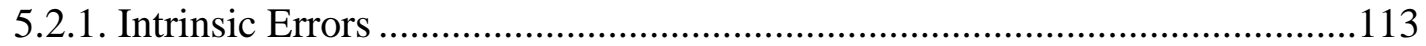

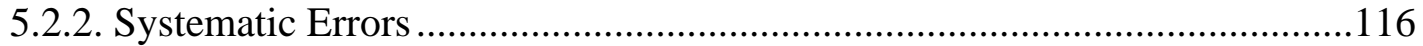

5.2.3. Incidental and Structural Failure.............................................................121

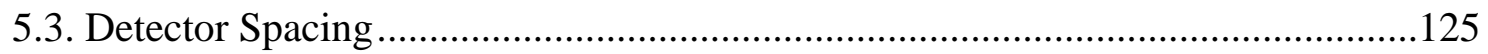

5.4. Travel Time Posting Configuration .......................................................................126

5.4.1. Travel Time Updating Frequency ………....................................................126

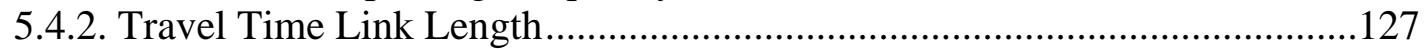

5.4.3. Posted Travel Time Range ........................................................................128

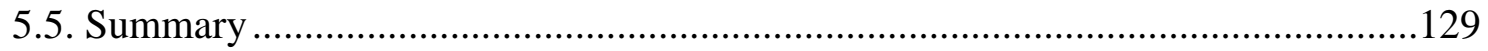

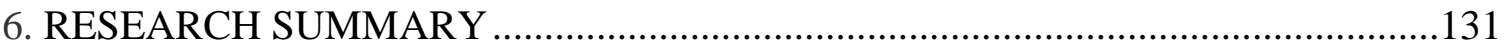

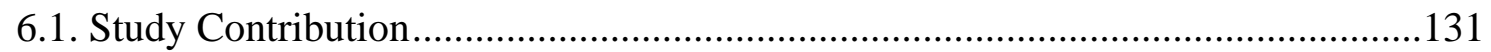

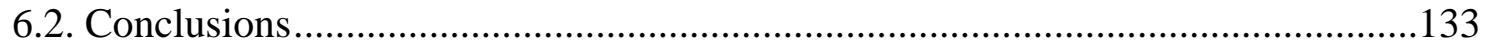

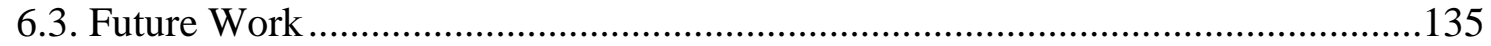

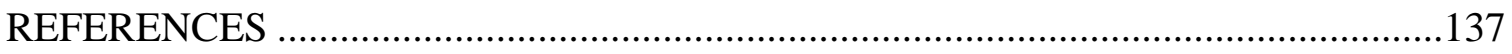

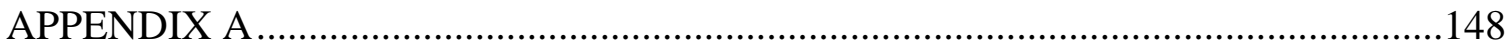

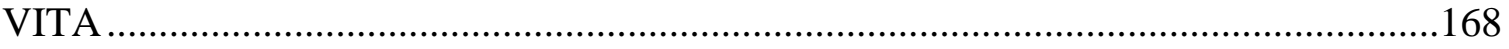




\section{LIST OF TABLES}

TABLE

PAGE

Table 3-1 Example of Detector Errors............................................................................ 52

Table 3-2 Rule-based Tests Used in the Data Filtering Step ……………………………... 56

Table 4-1 Accuracy and Reliability of Tested On-Line Travel Time Estimation

Methods for Simulated Uncongested Condition................................................................. 89

Table 4-2 Accuracy and Reliability of Tested Off-Line Travel Time Estimation Methods for Simulated Uncongested Condition. 90

Table 4-3a Accuracy and Reliability of Tested On-Line Travel Time Estimation Methods for Simulated Incident Scenario 1 between 7:00 A.M. and 9:00 A.M.

Table 4-3b Accuracy and Reliability of Tested On-Line Travel Time Estimation Methods for Simulated Incident Scenario 1 between 7:30 A.M. and 8:30 A.M.

Table 4-4a Accuracy and Reliability of Tested Off-Line Travel Time Estimation Methods for Simulated Incident Scenario 1 between 7:00 A.M. and 9:00 A.M. 95

Table 4-4b Accuracy and Reliability of Tested Off-Line Travel Time Estimation Methods for Simulated Incident Scenario 1 between 7:30 A.M. and 8:30 A.M. 96

Table 4-5 Accuracy and Reliability of Tested On-Line Travel Time Estimation Methods for Simulated Incident Scenario 2

Table 4-6 Accuracy and Reliability of Tested Off-Line Travel Time Estimation Methods for Simulated Incident Scenario 2.

Table 4-7 Accuracy of Tested On-Line Travel Time Estimation Methods for Real-world Cases 106

Table 4-8 Accuracy of Tested Off-Line Travel Time Estimation Methods for Real-world Cases

Table 5-1 Accuracy and Reliability of Travel Time Estimation Using Different Smoothing Methods....

Table 5-2 Results of Different Data Imputation Methods 112

Table 5-3 Impacts of Intrinsic Errors on Travel Time Estimation Performance ............. 116

Table 5-4 Tested Scenarios for Systematic Errors............................................................ 118 
Table 5-5a Impacts of Systematic Errors on Travel Time Estimation Performance for

Simulated Uncongested Conditions.

Table 5-5b Impacts of Systematic Errors on Travel Time Estimation Performance for

Simulated Incident Conditions.

Table 5-6 Impacts of Systematic Errors in Low Speed Measurements on Travel Time

Estimation Performance for Simulated Incident Conditions

Table 5-7 Impacts of Incidental and Structural Failures on Travel Time Estimation

Performance.

Table 5-8 Impacts of Detector Spacing on Travel Time Estimation Performance 126

Table 5-9 Travel Time Estimation Performances with Different Travel Time

Updating Frequencies

Table 5-10 Travel Time Estimation Performances with Different Travel Time Link

Lengths

Table 5-11 Travel Time Estimation Reliability with Different Posted Travel Time

Ranges.

Table A-1 Accuracy and Reliability of Travel Time Estimation Using Simple Moving Average

Table A-2 Accuracy and Reliability of Travel Time Estimation Using Exponential Moving Average 149

Table A-3 Results of Different Data Imputation Methods without Within-Station Imputation

Table A-4 Results of Different Data Imputation Methods with Within-station Imputation

Table A-5 Impacts of Intrinsic Errors on Travel Time Estimation Performance for Simulated Uncongested Conditions.

Table A-6 Impacts of Intrinsic Errors on Travel Time Estimation Performance for

Simulated Incident Case 1 between 7:30 A.M. and 8:30 A.M.

Table A-7a Impacts of Systematic Errors on Travel Time Estimation Performance for Simulated Uncongested Conditions without Data Filtering....

Table A-7b Impacts of Systematic Errors on Travel Time Estimation Performance for Simulated Uncongested Conditions with Data Filtering. 
Table A-8a Impacts of Systematic Errors on Travel Time Estimation Performance for Simulated Incident Conditions without Data Filtering

Table A-8b Impacts of Systematic Errors on Travel Time Estimation Performance for Simulated Incident Conditions with Data Filtering. 158

Table A-9 Impacts of Systematic Errors in Low Speed Measurements on Travel Time Estimation Performance for Simulated Incident Conditions 160

Table A-10 Impacts of Incidental and Structural Failures on Travel Time Estimation Performance for Simulated Uncongested Conditions 161

Table A-11 Impacts of Incidental and Structural Failures on Travel Time Estimation Performance for Simulated Incident Conditions.

Table A-12 Impacts of Detector Spacing on Travel Time Estimation Performance for Simulated Uncongested Conditions

Table A-13 Impacts of Detector Spacing on Travel Time Estimation Performance for Simulated Incident Conditions. 163

Table A-14 Travel Time Estimation Performances with Different Travel Time Updating Frequencies for Simulated Uncongested Conditions

Table A-15 Travel Time Estimation Performances with Different Travel Time Updating Frequencies for Simulated Incident Conditions. 164

Table A-16 Travel Time Estimation Performances with Different Travel Time Link Lengths for Simulated Uncongested Conditions 165

Table A-17 Travel Time Estimation Performances with Different Travel Time Link Lengths for Simulated Incident Conditions 165

Table A-18 Travel Time Estimation Reliability with Different Posted Travel Time Ranges for Simulated Uncongested Conditions 166

Table A-19 Travel Time Estimation Reliability with Different Posted Travel Time Ranges for Simulated Incident Conditions 167 


\section{LIST OF FIGURES}

FIGURE

PAGE

Figure 2-1 Schematic Diagram of Detector Configuration............................................ 26

Figure 2-2 Schematic Diagram of Space-Time Sub-region......................................... 30

Figure 2-3 Schematic Diagrams for Traffic Dynamics Approach................................. 34

Figure 3-1 Travel Time Estimation Framework ........................................................ 49

Figure 3-2 Example of Microwave Detectors......................................................... 50

Figure 3-3 Example of Detector Data File............................................................. 51

Figure 3-4 Sketch Diagram for Spatial Imputation.................................................. 60

Figure 3-5 Clustering Results for a Detection Station on SR-826................................ 65

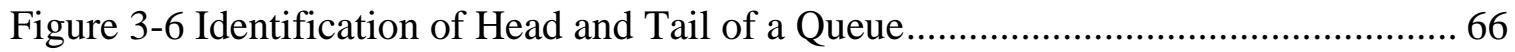

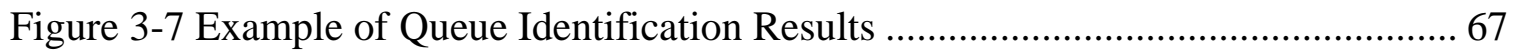

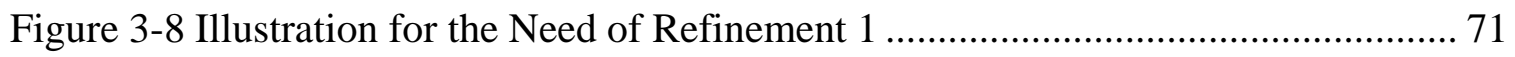

Figure 3-9 Illustration for the Need of Refinement 2 .............................................. 72

Figure 3-10 Schematic Diagram for Off-Line Travel Time Estimation ......................... 76

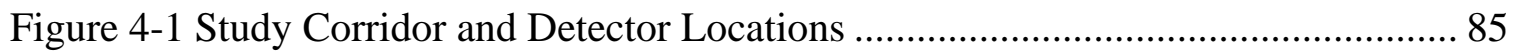

Figure 4-2 Estimated Travel Time for Simulated Uncongested Condition ...................... 88

Figure 4-3 Estimated Travel Time for Simulated Incident Scenario 1.......................... 92

Figure 4-4 Comparison of On-Line and Off-Line Estimation Methods ......................... 97

Figure 4-5 Estimated Travel Time for Simulated Incident Scenario 2........................ 100

Figure 4-6 Estimated Travel Time for Real-world Case ............................................ 105

Figure 5-1 Illustration of Normal Distribution for Intrinsic Errors ............................. 115

Figure 5-2 Estimated Free Flow Speed for Detection Station DS-1513E ..................... 117

Figure 5-3 Examples of Incidental and Structural Failures ....................................... 124 


\section{CHAPTER 1}

\section{INTRODUCTION}

\subsection{Background}

As a measure of traffic congestion levels, travel time information is important to travelers as well as to transportation planners and operational personnel. Travelers can make informed trip decisions regarding their departure time, route, and travel mode with the provided travel time information. Transportation planners can better estimate travel demand given the knowledge of accurate link travel time, resulting in more reasonable designs of roadway alternatives and Intelligent Transportation Systems (ITS) deployments. Traffic operation personnel can easily identify the incidents and traffic congestions based on the estimated travel times, and will also be able to effectively evaluate the performance of current and historical traffic operations.

Traditional travel time measurement methods, including test vehicles and license plate matching, can only provide limited travel time data at a high expense. With the advancement of ITS technologies, however, new probe vehicle techniques such as License Plate Reader, Automatic Vehicle Identification (AVI) and Automatic Vehicle Location (AVL) technologies are starting to be used for travel time measurement. Nevertheless, these techniques generally have high implementation costs and also greatly rely on the market penetration of ITS devices. In parallel, point traffic detectors, such as loop detectors and Remote Traffic Monitoring Sensor (RTMS), are widely deployed along the roadways. The traffic measurements reported by these detectors provide a cost-effective way to indirectly estimate travel time. According to the survey of Kothuri 
et al. (2007), a total of 25 metropolitan areas were providing travel time, and 17 other areas were planning to provide travel time information in 2007. Most of the posted travel time estimates were based on speed measurements by point detectors. How to estimate travel time accurately and reliably based on point detector measurements is thus an important subject that is receiving increasing interests from transportation engineering researchers and practitioners.

\subsection{Problem Statement}

Travel time studies based on point detectors can be categorized as travel time estimations and predictions. Travel time estimation aims at calculating the vehicle travel time along a route based on current traffic conditions, i.e., known vehicle speed, volume, and occupancy. On the other hand, travel time prediction focuses on the calculation of future travel time based on unknown but predicted traffic conditions. Although some studies suggested that predicted travel time values should be used for the Advanced Traveler Information System (ATIS) applications (Vanajakshi 2004; Shen 2008), in practice, most Traffic Management Centers (TMCs) are still disseminating estimated travel time on Dynamic Message Signs (DMS) and traveler information systems such as 511 phone services and web services. Also, the estimated travel time is usually used as the basic input for travel time prediction in time series analyses (Guin 2006) and for training artificial intelligence applications to the prediction problems such as Artificial Neural Networks and Support Vector Regression (Van Lint 2004; Vanajakshi 2004; Shen

2008). Thus, the accuracy of estimated travel time directly affects the performance of 
travel time prediction. This study only focuses on freeway travel time estimations, especially in real-time applications.

Existing point detector-based freeway travel time estimation methods can be generally classified into two types: speed-based methods (also referred to as the trajectory-based methods and extrapolation methods) and the flow-based methods. The speed-based methods construct the trajectory of speed along a roadway based on point measurements of speed by traffic detectors and use this information to estimate the travel times. The flow-based methods use volume and/or occupancy measurements as input variables instead of using speed measurements. Previous studies suggest that each type of methods may perform differently under different conditions. For example, speed-based methods produce acceptable results at lower levels of congestion, but there are questions regarding their ability to produce accurate and reliable estimates of travel times under recurrent and non-recurrent congested conditions. Flow-based methods perform well for congested conditions, but they may not be appropriate for uncongested conditions. The literature review shows that none of these methods can provide satisfactory travel time estimates under all traffic conditions. Thus, hybrid travel time estimation approaches using a combination of estimation methods have been proposed as means of estimating travel time under different congestion levels. However, previous hybrid approaches either specify the application scenario for each estimation method or predefine a constant threshold for selecting estimation methods. Further, these hybrid models were tested for very short links and limited traffic conditions. The hybrid approaches have not been adequately explored and compared with existing methods. 
Even more important, these approaches have not well addressed the travel time estimation during fast changing incident conditions, which is critical to TMC operations.

In addition, various factors may affect the performance of travel time estimation, such as data preprocessing, detector errors, detector spacing, travel time link length, travel time updating frequency, and so on. The impacts of these factors on the accuracy and reliability of travel time estimation have not been extensively studied and require further investigation.

\subsection{Research Goal and Objectives}

The goal of this research is to develop and assess models to estimate freeway travel time based on point traffic detectors during non-congested conditions, recurrent congestion, and incident conditions. The study will assess the developed models and examine their performances in terms of accuracy as well as reliability. The specific objectives of this research are as follows:

1) Review the existing travel time estimation and prediction methods.

2) Develop detector data preprocessing procedures, including data filtering, data smoothing and aggregation, and data imputation.

3) Develop on-line and off-line hybrid travel time estimation models.

4) Define performance measures for travel time estimation accuracy and reliability, and compare the developed estimation models to existing models by using simulation as well as real-world travel time data to assess performance. 
5) Identify the main influential factors on travel time estimation and quantify their impacts on estimation performance.

\subsection{Dissertation Organization}

This dissertation is organized into six chapters. Chapter 1 introduces the research background, describes the problems to be solved, and sets the goal and objectives to be achieved.

Chapter 2 presents an extensive literature review regarding travel time data collection techniques, point detector data preprocessing procedures, existing travel time estimation and prediction methods, and their influential factors. The main purpose of this review is to understand the state-of-the-art of travel time estimation, especially the advantages and disadvantages of existing estimation methods.

Chapter 3 describes the framework of the proposed hybrid approaches for freeway travel time estimation based on point traffic detector measurements. This includes data acquisition and preprocessing, traffic condition identification, and travel time estimation models. Two on-line hybrid travel time estimation models as well as two corresponding off-line hybrid estimation models developed in this study are described herein.

Chapter 4 defines accuracy and reliability measures to evaluate the performance of travel time estimation methods. Existing speed-based methods, flow-based methods, and developed hybrid models are assessed using simulated travel time data as well as real-world data for different traffic conditions. Further, off-line estimation models are compared with their on-line counterparts to determine the potential improvement in the estimation accuracy and reliability when using travel time prediction. 
Chapter 5 identifies the main influential factors on freeway travel time estimation and analyzes their impacts on the estimation accuracy and reliability based on sensitivity analyses.

Finally, Chapter 6 summarizes the main contributions, draws conclusions, and recommends issues for future research. 


\section{CHAPTER 2}

\section{LITERATURE REVIEW}

This chapter presents a detailed review of the current practices in travel time data collection, point detector data preprocessing, travel time estimation and prediction methods, and the study of associated influential factors.

\subsection{Travel Time Data Collection Techniques}

Travel time information can be either directly measured or indirectly calculated from distance, speed, flow and other measurements. Direct travel time measurement technologies include test vehicles, license plate matching, and Intelligent Transportation System (ITS) probe vehicles (Turner et al. 1998). Indirect methods vary with the type of point detectors and the algorithms used to estimate travel time.

\subsubsection{Direct Measurement Techniques for Travel Time}

The test vehicle method is a traditional technique for the collection of travel time data.

The test vehicles are driven along specific routes that allow the recording of the cumulative travel time at each predefined point, which is in turn used to obtain the travel time on each segment and along the entire route. The drivers are instructed to drive with one of three different strategies (Roess et al. 2004). With the floating-car strategy, the drivers pass as many vehicles as those that pass the test cars so that they can maintain their relative position in the traffic stream and approximate the behavior of an average vehicle. The drivers following the maximum-car technique are instructed to drive as fast as they can only if the speed is within the safe or reasonable design range. The 
average-car technique requires the test car drivers to drive at the average speed level. The test vehicles may be instrumented with stop watch, tape recorder, or personal computer for the manual recording of the elapsed time; equipped with an electronic Distance Measuring Instrument (DMI) that is connected to the transmission of vehicles; or utilize a Global Positioning System (GPS) (Turner et al. 1998). The test vehicle techniques have relatively low initial costs. However, these techniques are time-consuming and their limited sample sizes may cause uncertainty in the measured travel time. The individual test driver may also inadvertently introduce bias to the measurement results.

License plate matching techniques collect the license plate numbers and their corresponding arrival time at various locations, and then match them between two consecutive locations. This information is further used to deduce the average travel time on each roadway segment. The license plate numbers can be collected either manually using a pen or pencil, or automatically with the help of video image processing. Compared to the test vehicle techniques, the license plate matching method can collect travel time from a larger sample of drivers, but requires more effort to read and match the license plates, and the results are sensitive to license plate reading errors (Turner et al. 1998; Courage et al. 1998).

Vehicles equipped with Automatic Vehicle Location (AVL) or Automatic Vehicle Identification (AVI) equipment such as a Global Positioning System (GPS), Bluetooth, or electronic toll collection tags, can be considered as ITS probe vehicles for travel time collection (Turner et al. 1998). These vehicles can be personal cars, public transit, and commercial vehicles (Wunnava et al. 2007; Vanajakshi et al. 2008). The use of ITS 
probe vehicle techniques for data collection can cover a wide range of roadway segment and time horizons with no disruption to the traffic. However, these techniques generally have high implementation costs, and greatly depend on the market penetration of ITS equipment and the accuracy of the tracking technology. Privacy issues are also an important concern for this type of technique.

\subsubsection{Indirect Measurement Techniques for Travel Time}

Travel time can be indirectly measured by either developing estimation algorithms based on point traffic measurements such as speed, flow and occupancy, or matching vehicles based on their images or signatures captured at two specific locations.

Various point detector technologies have been applied to measure traffic flow parameters. Generally, these point detectors are classified as intrusive detectors and non-intrusive detectors. Intrusive detectors are detectors embedded in the pavement or attached to the surface of the roadway. Examples of intrusive detectors include the inductive loop detector, magnetic detector, and etc. Non-intrusive detectors are installed on the roadside or above the roadway surface with minimum disruptions to the traffic. Microwave radar, infrared, ultrasonic, acoustic detectors, and video image processing are examples of non-intrusive detectors. In this section, a brief review is given to only the most widely used detectors for indirect travel time measurement. The detailed travel time estimation algorithms for these detectors will be discussed in a later section.

The inductive loop detector is one of the most popular data sources for indirect travel time estimation. When a vehicle passes through the loop, its engine acts as a conductor and absorbs the electromagnetic energy generated by alternating currents 
through the wire loop. This in turn reduces the loop inductance and increases the frequency of oscillator (an energy source for alternating currents). By detecting the changes in oscillator frequency over the preset threshold, the presence or passage of a vehicle is signified. The activation time, starting from a vehicle entering the detection zone to the vehicle leaving the detection zone, is referred to as vehicle occupancy time. Two types of loop configurations are commonly used, namely, the single loop detector and the dual loop detector (i.e., speed trap). While a single loop detector configuration must rely on the measurement of traffic volume and occupancy to calculate speed, dual loop detectors not only directly measure traffic volume and occupancy, but directly measure speed as well. Moreover, inductive loop detectors have the advantages of low capital costs, mature technology, proven accuracy, and the ability to function under all weather conditions. However, they also have the disadvantage of unreliability due to detector failures and frequent maintenance.

Microwave radar sensors transmit the signals in the format of either continuous Doppler waves or continuous frequency modulated waves. As these transmitted waves encounter the vehicles, they are reflected back. Detecting the frequency changes in the transmitted and received signals allows for the measurement of vehicle and traffic information. The continuous frequency modulated microwave radar can detect speed, vehicle count, and presence, as well as the passage of vehicles. The Doppler microwave radar cannot detect the stopped vehicles as the Doppler wave frequency does not shift with zero speed. Thus, Doppler radars are used mainly to measure speed. Compared with inductive loop detectors, microwave radar detectors have a longer life, lower life 
cycle cost, and require less frequent maintenance, but they also have higher installation costs and special height requirements for the mounting location.

Video image detectors (VID) consist of cameras, processors and software. The images captured by cameras are digitized and analyzed by the processor. The changes in image background are identified and converted to traffic parameters by the software. The information gathered by video image detectors includes vehicle presence, passage, vehicle length for use in classification, speed, and in some products incident occurrence can be. However, the installation of cameras requires careful consideration of mounting height, location, and stability, as well as such issues as the periodic cleaning of lenses. Moreover, the performance of video image detectors is affected by inclement weathers such as rain, fog, and snow.

In addition to the above mentioned technologies, vehicle signature matching using point detectors is a newly emerging method for the measurement of travel time and speed. The matching is based on unique vehicle features, such as color image (Dubuisson et al. 1993), specific vehicle length (Coifman 1998; Coifman and Cassidy 2002; Coifman and Ergueta 2003), or features obtained from the detector frequency detuning curve including shape parameter, vehicle length, degree of symmetry, maximum magnitude, and number of high magnitude (Ritchie et al. 2005). By capturing these unique features and matching them at two consecutive locations, the travel time on the link can be measured. The capabilities of inductive loop detectors, laser sensors, weigh-in-motion (WIM) sensors, and video cameras to capture vehicle-unique features have been tested in previous research (Turner et al. 1998). Vehicle signature matching techniques generally perform well under both free-flow conditions and congested conditions, but the 
measurement error may increase during free-flow conditions due to reduced detection resolutions at low traffic flow levels (Coifman 1998; Coifman and Cassidy 2002; Coifman and Ergueta 2003; Jeng et al. 2007; Ndoye et al 2008). Another issue is that the existing vehicle signature matching techniques are usually based on lane-by-lane matching and therefore require a low lane changing rate and relatively constant vehicle position in platoon to achieve higher matching rate.

\subsection{Data Preprocessing}

The quality of detector data greatly influences the accuracy and reliability of travel time estimation. Without accurate data, even with significant improvement in travel time estimation algorithms, the estimated travel time is still not accurate. It is therefore necessary to preprocess the data before applying them to the travel time estimation

process. In this section, the type of errors in detector data are identified, followed by a discussion of the data filtering and data imputation procedures.

\subsubsection{Errors in Detector Data}

Payne et al. (1976) categorized loop detector malfunctions into five types: sensor stuck on/off; chattering; pulsing; hanging; and intermittent malfunctioning. A sensor stuck on/off situation may produce similar detector measurements as those during incidents or special events. When the detector is stuck at the "on” position, zero speed, zero volume, and an occupancy of $99 \%$ are consistently reported. On the other hand, zeros are reported for all three parameters when it is stuck "off”. During chattering, the detector produces random outputs reflecting short duration pulses, which result in the abnormally high or low values of traffic parameters. For example, incorrect volume count will 
occur when the detector pulsing produces more than one pulse for the presence of the same vehicle. Detector hanging occurs when the detector remains at the state of "on" or "off" for a long time. In this case, hanging "on" may produce the results of high occupancy with the same volume and decreasing speed, while hanging "off" generates the reverse results. Extremely low or high traffic volumes may indicate intermittent malfunctions. In addition to these errors identified by Payne et al. (1976), cross-talk from adjacent lanes is also a common error for inductive loop detectors. With cross-talk, vehicles in adjacent lane sometimes actuate a loop when there is no vehicle over that loop (Middleton and Parker 2002).

Three types of loop detection failures were identified by Van Lint (2004): incidental (or occasional), structural, and intrinsic. Although incidental failures happen randomly, various factors may contribute to their occurrence, such as a temporary transmission failure resulting from power outages. Structural errors are usually caused by detector malfunctions or mis-calibrations. With detector malfunctions, abnormal data may be collected, or a wide range of data may be missing. A consistent bias may exist in the measurement results if the detector is miscalibrated. Finally, intrinsic failure corresponds to measurement noise, which is inherent to the detector type under consideration.

Coifman (2004) assessed the performance of four models of loop sensors and the Remote Traffic Microwave Sensor (RTMS) deployed along I-80 in California. The study found that most of the errors occur due to lane changes over the detection zones. The identified error types were over-counting non-flicker (i.e., detection of a vehicle outside the detection zone), over-counting flicker (i.e., detector turns off and back on 
when detecting a vehicle), short on-time, under-count when not detecting vehicles, signal drop out in the middle of semi-trailer trucks, and missed motorcycle. This study also shows that the variations in RTMS detection zone size across different lanes causes the systematic errors in nearest lane measurement due to small detection zone and farthest lane measurement due to occlusion. Denning (2007) identified two major sources of errors in radar detector measurement for the Ohio Department of Transportation's (ODOT) Columbus Traffic Management Center: the cosine effect and the detection of occluded vehicles. The cosine effect emerges when the path of a measured vehicle is not in the same line as the axis of the radar's emitted wave. This effect results in the measured speed being only a projection of the actual speed in the direction that the radar antenna is pointed. When the view of a vehicle is blocked by a large vehicle in nearby lanes, the radar detector cannot detect the occluded vehicle and thus reports no measurement.

\subsubsection{Data Filtering}

Extensive research to eliminate faulty detector data and improve data quality has been reported in the literature. These studies can be classified into two levels: microscopic and macroscopic. Microscopic level screening diagnoses detector errors by processing raw loop detector signals or the sensor ON/OFF indications. In contrast, macroscopic level detection checks temporal aggregated data (Payne and Thompson 1997; Vanajakshi 2004; Lu et al. 2008).

Microscopic level screening is the most direct way to diagnose detector errors. This type of screening can differentiate the detector faults from other system problems, 
such as communication failures (Lu et al. 2008). Error detection at this level usually varies with the type of sensor and requires a certain modification or reprogramming of the devices (Vanajakshi 2004). Chen and May (1987) compared the on-time of vehicles passing through the detection zone with the average on-times for a detector or a station to identify a faulty single loop detector. Jacobson et al. (1990) developed an error detection algorithm based on the thresholds for volume-to-occupancy ratio. Coifman (1999) identified the detector with errors based on the fact that the same vehicle passing though each loop of dual loop detector should have an identical on-time under free flow conditions. Ametha et al. (2001) developed an algorithm for comparing the average vehicle length against the calibrated range of effective vehicle lengths to detect the errors. The average vehicle length estimation was based on the on- and off- times over paired loop detectors. May et al. (2005) proposed and assessed nine microscopic detector diagnostics based on 1/60s loop detector data, including detector activity tests, static and dynamic minimum and maximum on-time, mode on-time, dual detector on-time difference, minimum off-time, and dynamic maximum off-time.

Compared to the microscopic level detection, the macroscopic approach is more widely used due to the availability of aggregated data. Most of the studies at this level use thresholds to check the validity of detector data. Minimum and maximum thresholds are commonly applied for each measurement of speed, volume, and occupancy. If the data fail any one of these thresholds, the data is considered invalid. The commonly used minimum values are zeros for all three parameters. The used maximum threshold for volume is 5000 vphpl for 30-second volume, or 2400 or 3000 vphpl for 20 -second volume. The maximum occupancy values are $90 \%$, 95\%, or $100 \%$. 
The used maximum speed is 100 vph (Payne et al. 1976; Quiroga et al. 2005). Chen et al. (2003) also limited the number of samples with zero occupancy to be less than 1200 per day, and the number of samples with occupancy greater than $35 \%$ to be less than 200 per day, based on the idea that traffic cannot be congested for most of the day.

Even when individual speed, volume, and occupancy pass the threshold tests, the combination of these parameters may violate the basic traffic flow principles. Nihan et al. (1990) investigated single loop detector data and developed acceptable ranges of volume/occupancy ratio for a given occupancy measurement. However, the boundaries of the acceptable ranges have to be calibrated based on historical data. Vanajakshi (2004) and Turner (2007) examined detector data based on the fundamental traffic relationship among speed, volume, and density. The average effective vehicle length is also an important parameter that was checked in previous studies. Turner et al. (2004) limited the ranges of the average effective vehicle length to between 9 and $60 \mathrm{ft}$, while Al-Deek et al. (2004) used between 10 and $60 \mathrm{ft}$ as a reasonable range for this length.

In addition to data filtering at one location and one timestamp, temporal and spatial variations of detector data are also indicators of possible errors. Temporal tests can consist of either a temporal variability check or temporal consistency check (University of Florida Transportation Research Center 2005). Temporal variability thresholds are used to check whether identical data are reported for consecutive time periods. Turner (2007) set the maximum consecutive number of periods with constant values to be 30 minutes to indicate a detector failure. A study by the University of Florida Transportation Research Center reduced the threshold from 5 to 15 minutes depending on the time of day (2005). For a daily-based check, Chen (2003) proposed 
the usage of entropy to test if the occupancy data are constant for most of the time. If the entropy of occupancy is less than four, then the detector data is invalid. A similar entropy-based test was used by Al-Deek et al. (2004) when building the central Florida data warehouse. The temporal consistency check examines whether there exists an abrupt change in measured values for two consecutive time periods. The ranges of -15 to 15 veh, -40 to $30 \mathrm{mph}$, and $-40 \%$ to $50 \%$ were recommended for the change of volume, speed, and occupancy, respectively, between two consecutive 30-second detector data in a previous study (University of Florida 2005).

Spatial consistency test checks the changes in measurements between neighboring lanes and between detectors at upstream and downstream stations. Lane deviation tests have been conducted by Payne et al. (1976) to identify the lane with too high or too low occupancy. According to this study, if the lane occupancy at the five-minute aggregation level is more than twice the average value for all lanes or less than three times the average value, the detector is declared to malfunction. A study by the University of Florida (2005) suggested a volume count change of -10 to 10 veh, a speed change of -20 to $20 \mathrm{vph}$, and an occupancy change of $-20 \%$ to $20 \%$ between measurements of lane detectors at the same location. For detectors at neighboring stations, these values plus 20d for count change, and 40d for speed and occupancy change, where $d$ is the distance between neighboring stations in units of miles, are allowed.

The above suggested threshold-based tests are either performed at the raw 20- or 30-second level, or at an aggregated level. For example, the tests conducted by Turner (2007) are all based on raw detector data. However, other studies, such as those by 
Payne et al. (1976) and Al-Deek et al. (2004), stated that the relationship to estimate the average effective vehicle length is not linear and that the data which satisfy this criterion at the raw data level may fail the test at the aggregated levels. Therefore, they separated the data filtering tests into two levels: one is the 20- or 30-second level mainly focusing on the individual traffic parameters or a simple combination of these parameters, the other is at the aggregated levels (for example, 5-minute aggregation) for more complicated tests.

Instead of using threshold methods for the data validity check as discussed above, other studies applied statistical or data mining methods to eliminate invalid data. Kondagari (2006) constructed probability distributions of each individual traffic parameter and their combinations based on historical data. These distributions were used in real-time to obtain the probability of each measurement or each combination, which were further compared against predefined thresholds to identify erroneous data. Since the developed probability distributions are not exclusive, this method may lead to conflicting conclusions based on different distributions. Peeta and Anastassopoulos (2002) used Fourier transformation to detect erroneous detector data and correct them. However, this method still needs criteria to separate incidents from other faulty data since the Fourier transformation treats both as abnormal data. Oh and Park (2008) employed Wavelet transformation to eliminate noisy data. Similar to the Fourier transformation, this method may have difficulty in identifying incidents from the true erroneous data. 


\subsubsection{Data Imputation}

The 1992 Guidelines for Traffic Data Programs of the American Association of State Highway and Transportation Officials (AASHTO) did not recommend the imputation of missing data or erroneous data since this may introduce unquantifiable errors (AASHTO 1992). However, several studies have recommended that AASHTO should revise these guidelines and allow data imputation since imputation methods have been greatly improved. These improvements allow the original relationship among raw data to be preserved, the impacts of imputation to be measured, and reasonable accuracy to be achieved (Conklin and Scherer 2003; Nguyen and Scherer 2003; Ni et al. 2005; Zhong et al. 2006; Fernández-Moctezuma et al. 2009). Depending on the number of times that missing data are imputed, the imputation methods can be classified as either a single imputation or multiple imputation approach. Below is a brief discussion of these two types of data imputation.

The single imputation approach only imputes the missing data once. Since traffic data are time-dependent and vary from one location to another, the temporal relationship and/or spatial information can be employed to handle the missing data.

Archived historical data provides a good source for estimating this missing data. The historical mean method employs archived information to estimate and use the average value of a parameter at the same timestamp to fill in the missing value (Conklin and Scherer 2003; Nguyen and Scherer 2003). The optimal number of days for which the average is estimated has to be determined either by experience or sensitivity analysis. The historical mean method may be further improved by limiting the average to only the same weekdays or weekends. This approach is suitable for a long period of missing 
data and is easy to implement, especially for on-line applications. However, one disadvantage is that it reduces the variance of parameter values as more and more data are imputed. Moreover, it may not account for current abnormal conditions, thus resulting in large errors.

Instead of using historical values, some approaches focus on the temporally neighboring relations. For example, the "Roll-forward" approach replaces the missing data with the immediate preceding value (Fernández-Moctezuma et al. 2009). This technique is simple but inappropriate for cases in which a large number of data is missing. An improvement to this approach is to estimate the missing value by either averaging the data from surrounding time periods or by linear interpolation between these data (Conklin and Scherer 2003; Van Lint 2004). For off-line applications, both the past and future values can be applied, but for on-line implementation, only the preceding data are available for averaging. More elegant time series analyses were also applied for data imputation. Van Lint (2004) proposed the application of exponential moving average (EMA) for temporal imputation. Differing from the simple moving average, the EMA method is a weighted moving average, with more weights for recent data than older data. Additionally, more complicated time series models are the autoregressive integrated moving average (ARIMA) and its variations such as the seasonal ARIMA method (Williams and Hoel 2003; Nguyen and Scherer 2003). By differencing or seasonal differencing, a non-stationary time series is transformed to a stationary one that can be fitted. However, the EMA and ARIMA models have difficulty in recovering missing values when a high percentage of data is corrupted. Therefore, Wen et al. (2005) proposed the application of a grey time series model to recover temporal missing 
data. The core of this method is to define a new time series with the difference between two consecutive terms equal to the value in the original time series, and then to construct a group of differential equations for the new time series. The solution to these equations is used to forecast the missing value. As mentioned by the authors, this method does not require the knowledge of data distribution and needs only a minimum of four data points in a grey time series. In addition to the above traditional data imputation methods, Zhong et al. (2004) employed the genetic algorithm-based time delay neural network and local weighted regression, which showed better performance than the aforementioned methods.

The measurements from neighboring detectors can also provide inputs to the estimates of the corrupted values. Van Lint (2004) applied linear interpolation between upstream and downstream detector data to find the missing values. Linear interpolation is a simple method, but one drawback is that it makes a naïve assumption about the data (Chen et al. 2003). Therefore, based on historical data, the linear regression method was used to derive the relationship between the data of neighboring pairs. Al-Deek et al. (2004) argued that the relationship is not linear, and thus developed a pair wise quadratic model to estimate the missing values. However, this approach requires considerable effort, especially when the number of detectors is large, as the regression expression has to be established between the current detector and each neighboring detector, including the adjacent lanes at the same station, lanes at one station upstream, and one station downstream. Wen et al. (2005) applied a grey relational-based pseudo-nearest-neighbor method to handle the missing spatial values. More recently, a regression tree method was used by Logendran and Wang (2008) to predict the missing speed data, which took 
the flow, incident, weather, and time-of-day related variables into consideration. One issue with this multiple regression method is that it may not work when one of the independent variables is missing.

Instead of considering the temporal and spatial characteristics separately, most researches combine both temporal and spatial data imputations. For this purpose, Nguyen and Scherer (2003) developed the space-time autoregressive moving average models STARMA and C-STARMA. Compared to the traditional ARIMA method, the values forecasted by these two models are not only a function of past observations and errors at the same location, but also a function of the values at neighboring locations. The difference between the STARMA and C-STARMA models is that STARMA only uses neighboring detectors' data up to the previous time interval, but C-STARMA also includes the available spatial data at the same time interval. Imputation results from different methods were further combined using the linear regression model. Thus, more computational work is required for this modeling approach. Van Lint (2004) selected the minimum of temporal and spatial imputation as the final imputed value of speed which corresponds to the maximum travel time. Geng and Wu (2008) applied a time-series linear interpolation method to impute data with an assigned interval of less than five minutes and a weighted average method for those having an interval of greater than five minutes. The weighted average method was based on the correlation coefficients of temporal and spatial neighboring data. Specifically, the study used the correlation coefficient between two neighboring lanes at the same station, the coefficient between the same lane at upstream and downstream stations, or the correlation coefficient 
between temporally neighboring days at the same location (that is, the same weekday in different weeks) to find the weights and determine the imputed value.

Since there are various methods that can be used for data imputation, the question is which approach is more accurate or more appropriate. Conklin and Scherer (2003) examined five types of data imputation strategies by artificially removing certain traffic data points. These five types are historical average, average of neighboring time periods, average of neighboring stations plus the historical lane distribution, the expectation-maximization (EM) algorithm, and data augmentation. Comparing the imputed volume with the actual data, the study showed that the EM method performs better than the other four strategies. However, for those applications that do not require higher accuracy, the study concluded that the simple approach of using the historical average is also adequate. For the off-line travel time estimation, three approaches were tested by Van Lint (2004). The three approaches are the null replacement (leave the data as it is except at the spatial boundary where the data were replaced by the last known value), simple imputation through spatial and temporal interpolation, and a first order local weighted regression (LWR) model combined with an extended Kalman Filter. The comparison among these approaches indicated that the first two approaches outperform the more sophisticated model-based data imputation method. This was explained by the fact that the LWR/Kalman Filter method cannot track the onset of congestion due to the missing data, and that only one fundamental diagram was used for detectors positioned at different locations. For on-line travel time prediction, the null replacement, simple interpolation, exponential moving average, and a combination of the simple interpolation and exponential moving average were tested for incidental failure 
(Van Lint 2004). The results revealed that the last three methods are better than the null replacement method. For the structural failure, the null replacement was compared to the simple interpolation and it was found that simple interpolation can produce surprisingly good results (Van Lint 2004). Chen et al. (2006) tested the methods of historical average, temporal interpolation, spatial interpolation, hybrid time series and historical average, and artificial neural network for imputing missing volume data. The comparison showed that temporal interpolation is more accurate than other methods in all tested cases. The performances of the remaining methods vary with time-of-day, day-of-week, and missing percentages. Based on these results, this study developed a hybrid algorithm to impute the missing data by applying different methods for different time-of-day and day-of-week.

To account for uncertainty in the missing data, the multiple imputation schemes estimate the same missing value using different approaches and then calculate the mean and variance of the estimates. Generally, the multiple imputation approach has been found to perform better than single imputation (Wang et al. 2008). As one example of multiple imputation, Ni et al. (2005) applied the EM/DA (Expectation Maximization and Data Augmentation) approach for multiple times to the missing ITS data in the Georgia NaviGAtor system. The EM/DA method uses the EM results as an initial estimate for missing values. It then performs the regression over all the data. Next, the missing value is estimated by using the regression equation plus one random value from the residual distribution of the regression. These steps are repeated until the mean and covariance matrix converge. The results of this method showed a high imputation quality and low mean absolute percentage error with multiple imputation scheme. The 
approach used in this paper, however, may not work for situations in which the detectors fail to report data for a long period of time, since the basic assumption of the EM/DA approach is that the data is missing at random (i.e., the probability that a data is missing is independent of the absence of other data). Wang et al. (2008) also proposed two multiple imputation models: one directly imputes the missing travel time based on the information collected by the available detectors and travel time, while the other model estimates only the missing detector data. The key idea of these two models is to search the most similar historical cases using the k-nearest neighbor methods, which are in turn used to determine the distribution of missing variables. Once the missing values are replaced with the mean of this distribution, the distribution is reexamined and compared with the previous one. These steps are repeated until the mean and variance of the variable distribution converge. Compared to the work of Ni et al. (2005), these two models do not make the assumption that data is missing at random, but do require an extensive calibration of thresholds to categorize the traffic patterns and identify the similar cases.

\subsection{Travel Time Estimation}

The accurate and reliable estimation of freeway travel time based on point detector measurements is needed to support advanced traveler information systems (ATIS) and advanced traffic management systems (ATMS). Transportation agencies are increasingly updating travel time information on their dynamic message signs (DMS), traveler information telephone services (511), web sites, and other ATIS devices. Transportation agencies also use this information to support better traffic management. 
Existing travel time estimation methods based on point traffic detectors can generally be classified into speed-based methods, flow-based methods, and hybrid approaches. Below is a detailed review of these three types of methods.

\subsubsection{Speed-Based Methods}

Speed-based methods (also referred to as extrapolation methods or trajectory-based methods) construct the trajectory of speed along a roadway to estimate the travel times based on the point measurements of speed by traffic detectors. Different assumptions regarding the speed trajectory will therefore lead to different speed-based methods. To facilitate the discussion, Figure 2-1 presents a schematic diagram for detector configuration. As an example, two roadway segments and three detectors are included in this diagram. These detectors have point speed measurements of $S_{1}, S_{2}$, and $S_{3}$, respectively. The distances between the neighboring detectors are $L_{1-2}$ and $L_{2-3}$. Below is a brief description of existing speed-based travel time estimation methods.

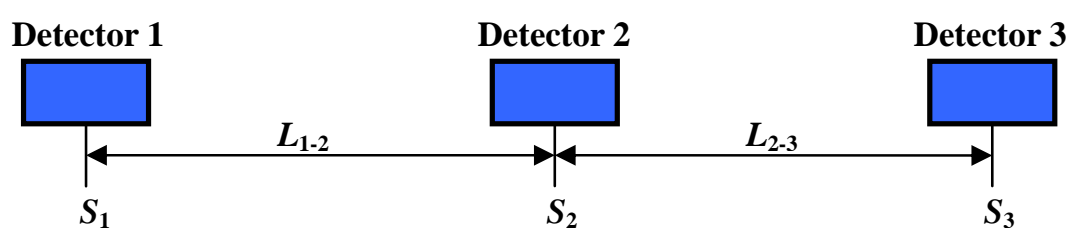

Figure 2-1 Schematic Diagram of Detector Configuration

\subsubsection{Point-to-Point Method}

A travel time link is composed of roadway segments. The segment travel times can therefore be used to estimate the total travel time along the link. The Point-to-Point method approximates the average segment speed by the speed detected at the upstream 
detector; therefore, the travel time $T T_{1-2}$ along the segment $L_{1-2}$ can be calculated as follows:

$$
T T_{1-2}=\frac{L_{1-2}}{S_{1}}
$$

The SunSuide software used in Florida allows the users to specify the length of influential area for each detector station associated with the travel time link. Thus, the user can configure the links to correspond with either the Point-to-Point method or the Mid-Point method described in the next section.

\subsubsection{Mid-Point Method}

The Mid-Point method assumes that each detector speed measurement represents the speeds of half distances to the next detector on both sides. The segment travel time is thus calculated as follows:

$$
T T_{1-2}=\frac{L_{1-2} / 2}{S_{1}}+\frac{L_{1-2} / 2}{S_{2}}
$$

The Mid-Point method is the most widely used travel time estimation algorithm in real-world systems, such as in Portland, Oregon (Kothuri et al. 2007), and in Florida DOT traffic management centers.

\subsubsection{Average Speed Method}

In the Average Speed method, the segment speed is approximated by the average of speed measurements at both ends of the segment. The corresponding expression for travel time estimation is listed as follows:

$$
T T_{1-2}=\frac{L_{1-2}}{\left(S_{1}+S_{2}\right) / 2}
$$




\subsubsection{Minimum Speed Method}

The speed used in the Minimum Speed method is the lowest value of speed measurements at either end of the roadway segment, resulting in the following expression for travel time estimation:

$$
T T_{1-2}=\frac{L_{1-2}}{\min \left(S_{1}, S_{2}\right)}
$$

This method was implemented in San Antonio, Texas (Kothuri et al. 2007; Kury 2008).

\subsubsection{Minnesota Algorithm}

A modified Mid-Point travel time estimation algorithm was developed by the Minnesota Department of Transportation (Mn/DOT) and applied to the Twin Cities. The speed is first estimated based on the volume and occupancy measurements from single loop detectors installed every half-mile, and then used for the estimation of travel time. Each roadway segment is divided into three regions. For each side region, the speed of the detector within that region is used. For the central region, the average speed of two adjacent detectors is applied. This results in the following expression:

$$
T T_{1-2}=\frac{L_{1-2} / 3}{S_{1}}+\frac{L_{1-2} / 3}{\left(S_{1}+S_{2}\right) / 2}+\frac{L_{1-2} / 3}{S_{2}}
$$

\subsubsection{Time Slice Model}

The Time Slice model considers the variation of speed with respect to time as vehicles traverse the roadway segment. For example, when a vehicle arrives at the second segment, the speed at the time $t_{0}+T T_{1-2}$ should be used if the entry time to the route is 
assumed to be $t_{0}$ ( $\mathrm{Li}$ et al. 2006). The corresponding mathematical expressions for travel times are the following

$$
\begin{aligned}
& T T_{1-2}=\frac{L_{1-2} / 2}{S_{1}\left(t_{0}\right)}+\frac{L_{1-2} / 2}{S_{2}\left(t_{0}\right)} \\
& T T_{2-3}=\frac{L_{2-3} / 2}{S_{2}\left(t_{0}+T T_{1-2}\right)}+\frac{L_{2-3} / 2}{S_{3}\left(t_{0}+T T_{1-2}\right)}
\end{aligned}
$$

This approach can be further improved by taking the travel time along the segment into consideration when applying the downstream speed, as follows:

$$
T T_{2-3}=\frac{L_{2-3} / 2}{S_{2}\left(t_{0}+T T_{1-2}\right)}+\frac{L_{2-3} / 2}{S_{3}\left(t_{0}+T T_{1-2}+T T_{2-3}\right)}
$$

As shown in Equation 2-8, the travel time for segment 2-3 has to be solved recursively.

\subsubsection{Piece-wise Linear Speed Model}

Figure 2-2 shows the space and time of a vehicle path divided into sub-regions for off-line travel time estimations. With the above mentioned speed-based methods, the speed is assumed to be constant in each sub-region but can have abrupt changes across the regions. To make such a transition smoother, Van Lint (2004) proposed the Piece-wise Linear Speed Based Model (PLSB). The basic idea behind this model is that the speed is assumed to be a linear function of space instead of a constant in each sub-region, that is, for a very small region $[x, x+\mathrm{d} x]$, the average speed would be calculated as follows:

$$
S(x)=S_{u}(k, p)+\frac{x-x_{u}}{x_{d}-x_{u}}\left[S_{d}(k, p)-S_{u}(k, p)\right]
$$


where $x_{u}$ and $x_{d}$ are the upstream and downstream detector locations of segment $k$, and $S_{u}(k, p)$ and $S_{d}(k, p)$ are the corresponding speeds during the time period $p$.

Based on this assumption, the relationship between time and space can be derived

as

$$
t(x)=t^{0}+\int_{x^{0}}^{x} \frac{1}{S(x)} d x=t^{0}+\frac{x_{d}-x_{u}}{S_{d}-S_{u}} \ln \frac{S_{u}+\frac{x-x_{u}}{x_{d}-x_{u}}\left(S_{d}-S_{u}\right)}{S_{u}+\frac{x^{0}-x_{u}}{x_{d}-x_{u}}\left(S_{d}-S_{u}\right)}
$$

and

$$
x(t)=x^{0}+\left[\frac{\left(x_{d}-x_{u}\right) S_{u}}{S_{d}-S_{u}}+x^{0}-x_{u}\right]\left[\exp \left(\frac{S_{d}-S_{u}}{x_{d}-x_{u}}\left(t-t^{0}\right)\right)-1\right]
$$

Note that the parameters $S_{u}, S_{d}, x^{0}$, and $t 0$ in Equations 2-10 and 2-11 are a function of space interval $k$ and time period $p$.

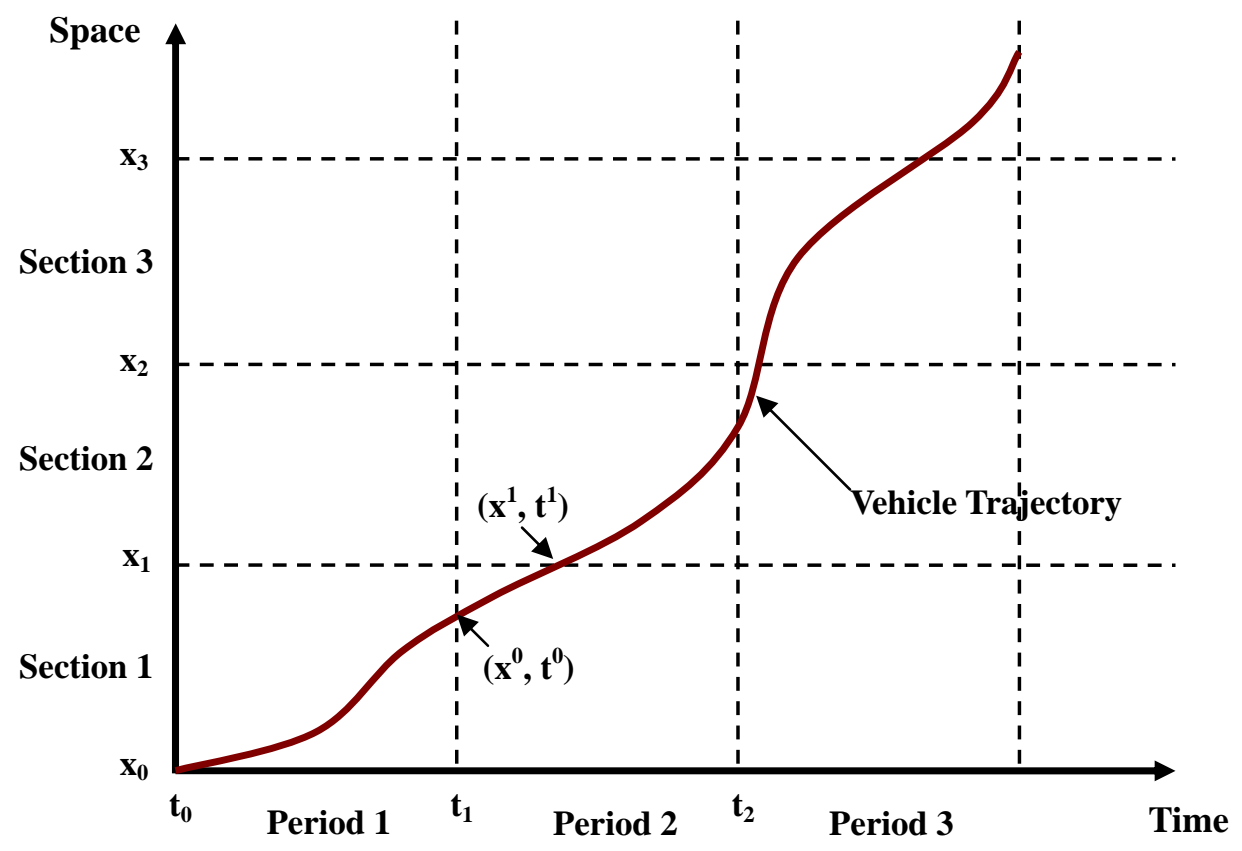

Figure 2-2 Schematic Diagram of Space-Time Sub-region 
To estimate the route travel time, a "Dynamic Network Level Travel Time Estimator” was used by Van Lint (2004). When a vehicle exits the subregion $(k, p)$, it may enter the region of $(k+1, p),(k, p+1)$, or $(k+1, p+1)$ depending on the exit point. This means that instead of using all of the speeds at the same time interval for route travel time estimation as for on-line applications, the speeds during the latest time periods may be applied. However, this dynamic route travel time estimation approach is only appropriate for off-line estimation; for on-line application, the speeds in the next time interval cannot be known in advance.

\subsubsection{Piece-wise Constant Acceleration Model}

The Piece-wise Constant Acceleration Based Model (PCAB) was proposed by Shen (2008) as an improvement to the PLSB model. The PCAB method assumes that the speed is a linear function of time (i.e., constant acceleration or deceleration rate) rather than a linear function of the distance, as is assumed by the PLSB model. The resulting average speed at time $t$ is,

$$
S(t)=S_{u}(k, p)+a\left(t-t^{0}\right)=S_{u}(k, p)+\frac{S_{d}{ }^{2}(k, p)-S_{u}{ }^{2}(k, p)}{2\left(x_{d}-x_{u}\right)}\left(t-t^{0}\right)
$$

and the corresponding location $x(t)$ is given as

$$
x(t)=x^{0}+\int_{t^{0}}^{t} S(t) d t=x^{0}+S_{u}\left(t-t^{0}\right)+\frac{S_{d}{ }^{2}-S_{u}{ }^{2}}{4\left(x_{d}-x_{u}\right)}\left(t-t^{0}\right)^{2}
$$

Again, the speeds, $S_{u}$ and $S_{d}$, are specific to each space interval $k$ and time period $p$. 


\subsubsection{Comparison of Speed-based Methods}

A number of studies have been conducted to evaluate the speed-based methods reviewed earlier in this section. Li et al. (2006) compared the performances of Mid-Point, time slice and PLSB models using AVI data based on toll tag and license-plate matching on two motorways in Melbourne, Australia. The comparison showed little difference between model performances, with all methods underestimating the actual travel time. In 2008, Shen evaluated the Mid-Point, Minimum Speed, Average Speed, PLSB, and PCAB models using the simulation. The results showed that both the PCAB model and Average Speed method achieve better accuracy than the PLSB model, and are much more accurate than the Mid-Point and Minimum Speed algorithms. Kury (2008) collected the travel time data on the I-195 corridor in Florida using the floating-car technique. The collected travel time was then compared to those estimated by methods including the Point-to-Point, Mid-Point, Minimum Speed, Minnesota algorithm, and those given by the SunGuide software. The comparison indicated that the average estimated travel time by the Point-to-Point method has a slightly lower percentage difference from the actual value compared to other algorithms, and that no statistically significant difference could be found between these algorithms under free-flow conditions. However, this study did not have enough data to compare these algorithms for congested traffic conditions. Similar to the results of Kury (2008), Kothuri et al. (2008) revealed that the San Antonio algorithm overestimates the travel time, while the other three algorithms achieve comparable performance. However, this paper did not mention under what conditions the testing was conducted, but stated that the higher error is associated with the transition 
period, such as the transition from congested to uncongested conditions, and suggested using the historical data for such a transitional region.

\subsubsection{Flow-based Methods}

The flow-based methods usually use volume and/or occupancy measurements as input variables to estimate travel time instead of using speed measurements. Examples of these methods include the traffic dynamics approach, shock wave method, and statistical-based method, as discussed below.

\subsubsection{Traffic Dynamics Approach}

The traffic dynamics approach (also referred to as the Cumulative Curve method and the N-D method) was first proposed by Nam and Drew (1996, 1999). This approach is based on characteristics of the stochastic vehicle-counting process and the principle of conservation of vehicles. Depending on how many vehicles can enter and exit the segment in a given time interval, i.e., the variable $m$, the travel time estimation can be separated into two models: one for normal conditions and one for congested conditions. The corresponding cumulative flow plots for these two conditions are illustrated in Figure 2-3. Note that the symbol $Q$ in this figure represents the cumulative volume count. For normal conditions, assuming first-in first-out, the total travel time for those vehicles that enter and exit the segment in the same interval can be estimated by the area between upstream and downstream cumulative curves. Dividing this total travel time by the corresponding number of vehicles yields the average segment travel time. A similar approach is applied for travel time estimation under congested conditions except that the 
total travel time calculation is for all the vehicles that enter the segment during time interval $\left(t_{n-1}, t_{n}\right)$.

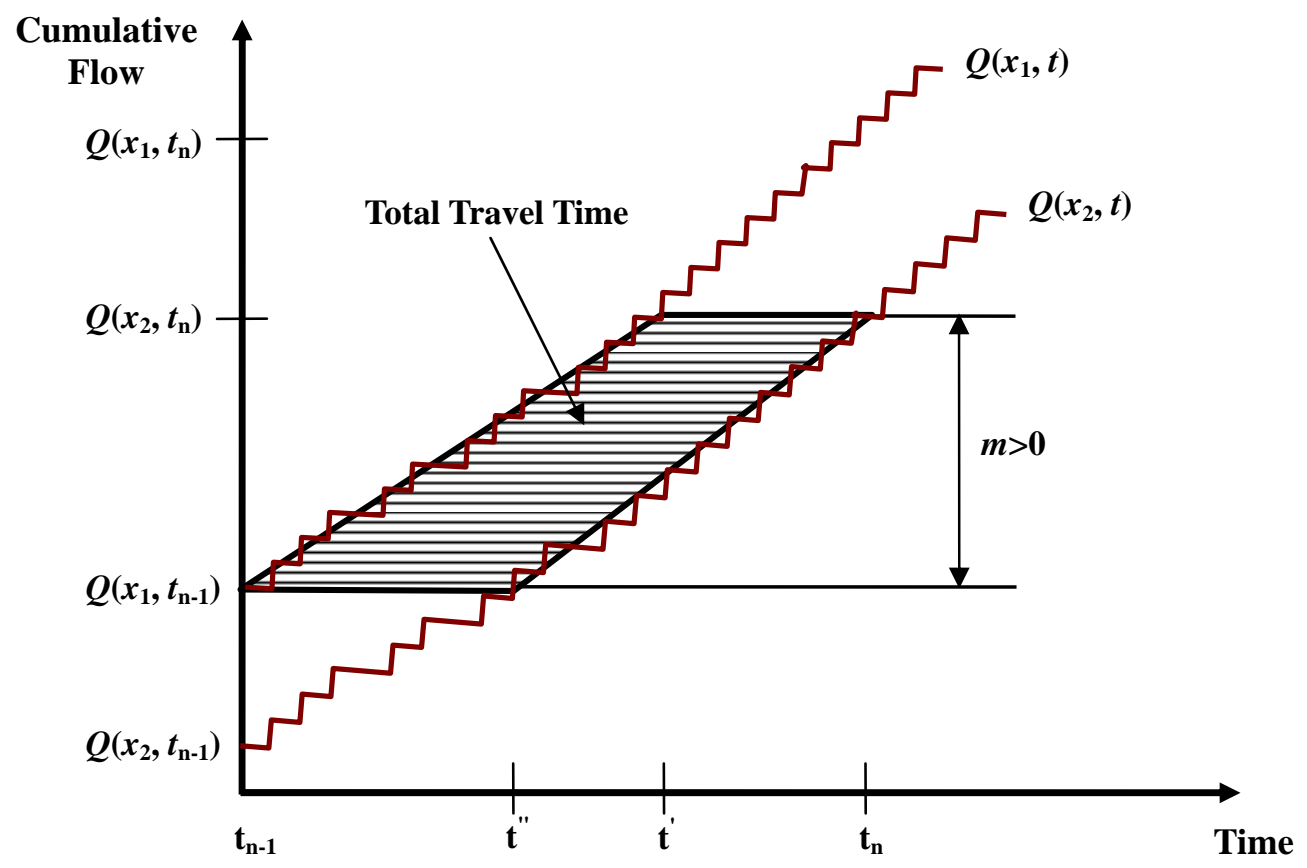

(a) Normal Conditions

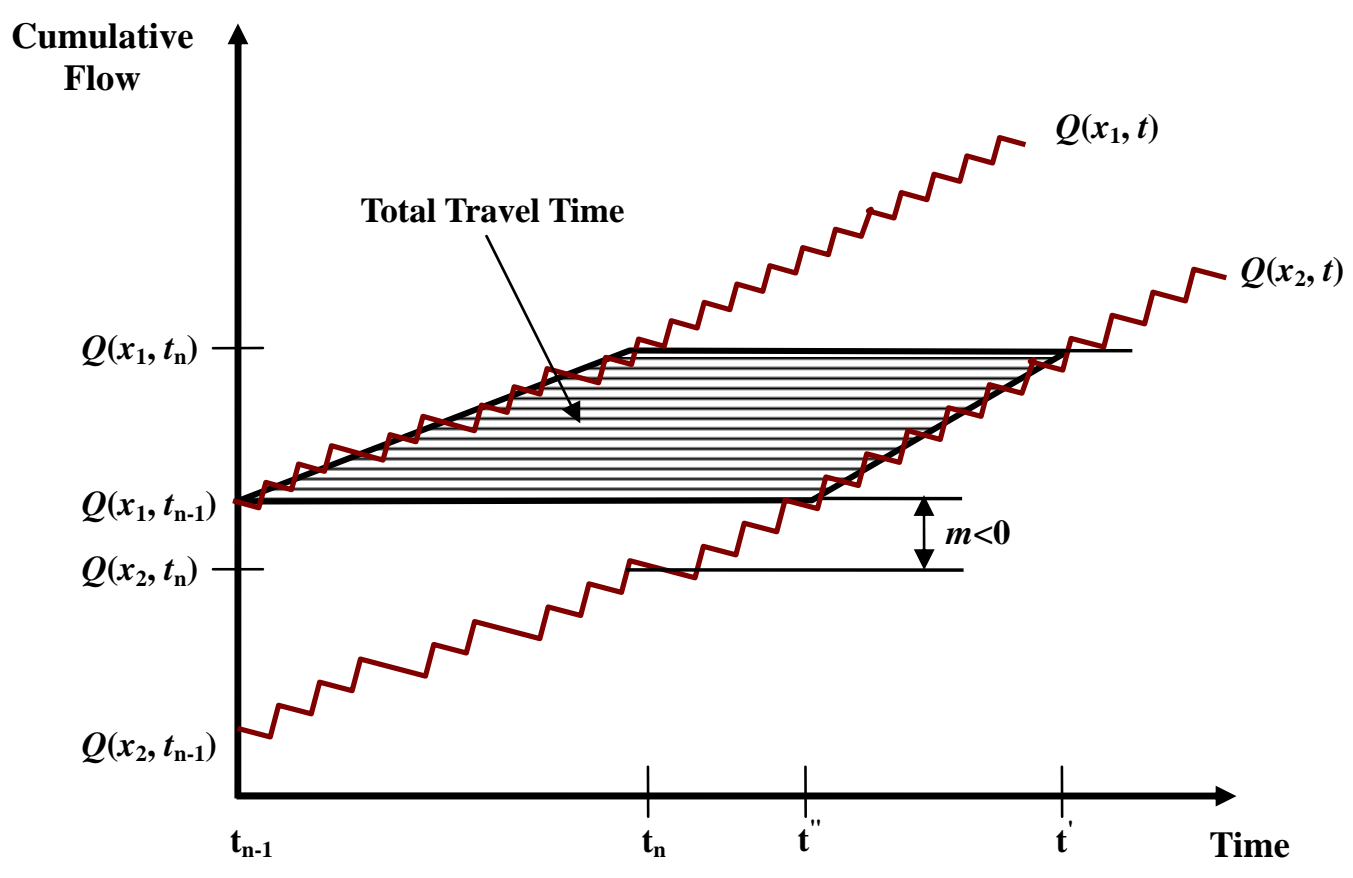

(b) Congested Conditions

Figure 2-3 Schematic Diagrams for Traffic Dynamics Approach 
Various improvements have been made to the original N-D method. Vanajakshi (2004) proposed the following four modifications:

- Use the Generalized Reduced Gradient optimization method to enforce the conservation of total number of vehicles;

- Reformulate the expression of travel time under normal conditions by taking all the vehicles into consideration, instead of only the vehicles that can enter and exit the segment in the same time interval;

- Use occupancy to calculate the density instead of using traffic counts; and

- Use the extrapolation method to estimate travel time during low volume conditions.

Zhang (2006) corrected the travel time estimation equation for congested conditions by considering the total travel time for those vehicles that exit the link during the time interval $\left(t_{n-1}, t_{n}\right)$ to account for the fact that under congested conditions, vehicles may spend more time on segments than the length of the time interval. This study also proposed the use of density, instead of variable $m$, as the threshold to determine whether the traffic conditions are normal or congested. Vanajakshi (2009) further proved that the two equations for normal and congested conditions can be unified under one expression, as follows:

$$
T T_{1-2}\left(t_{n}\right)=\frac{L_{1-2}}{2}\left(\frac{k\left(t_{n-1}\right)+k\left(t_{n}\right)}{q\left(x_{2}, t_{n}\right)}\right)
$$

where $T T_{1-2}\left(t_{n}\right)$ denotes travel time along a segment with a distance of $L_{1-2}, k\left(t_{n}\right)$ is the segment density at time $t_{n}$, calculated from occupancy based on a fixed average vehicle 
length, and $q\left(x_{2}, t_{n}\right)$ is the flow at segment downstream location during the time interval $\left(t_{n-1}, t_{n}\right)$.

\subsubsection{Shock Wave Method}

The shock wave method provides estimates of the shock wave speed and thus allows for the estimation of queue lengths during lane closure and incident conditions. When applying the shock wave method to travel time estimation, the travel time of vehicles within the queue depends on the queue discharge rate at the bottleneck location, while the travel time for vehicles outside the queue is estimated based on the measured flow and occupancy (Dhulipala 2002). Yeon and Ko (2007) compared the travel time estimated by both queuing theory and shock wave analysis with field data. The results showed that both methods underestimated the actual travel time for one freeway section, but produced results similar to the field data for another.

\subsubsection{Statistical Approach}

Statistics-based methods attempt to relate freeway travel times to traffic counts at upstream and downstream detector locations or to accumulated flows within the segments. The methods range from simple regression analyses (Liu and Chang 2006) and cross correlation analyses (Dailey 2003), to more complicated probabilistic regression models (Petty et al. 1998; Zhang et al. 1999; Guo and Jin 2006). Liu and Chang (2006) developed two regression models to estimate travel time: one for freeways with a permanent bottleneck, and another for freeways with a temporary downstream bottleneck. These two regression models relate the segment travel time to the accumulative flows at upstream and downstream detectors. The results seem promising 
even when the detectors are sparsely distributed. Dailey (1993) considered the average travel time as a deterministic variable, whose value was determined by maximizing the cross covariance function. Petty et al. (1998) proposed a probabilistic regression model that considers the average travel time along a link to be a random variable whose probability density function is the same as the arrivals at the upstream detector location. A regression analysis over the upstream and downstream flow measured during a given estimation window was used to determine this probability density function. The case study showed that this method can estimate travel time even during very congested conditions. Later, extensions of this method include using the B-splines, exponential moving (Zhang et al. 1999), and correlation analysis (Guo and Jin 2006) to improve the estimation of the probability density function.

\subsubsection{Hybrid Approaches}

Speed-based methods are widely used at Traffic Management Centers (TMCs) due to their simplicity. These speed-based methods may produce acceptable results at lower levels of congestion, however, there have been questions regarding their ability to produce accurate and reliable estimates of travel times under recurrent and non-recurrent congested conditions ( $\mathrm{Li}$ et al. 2006; Kury 2008; Kothuri et al. 2008). This is because many detection technologies are not able to measure speed accurately under congested traffic conditions. In addition, the speed measurements made at point locations may not reflect the speeds along the segment that they are supposed to represent. On the other hand, some flow-based methods rely on assumed average effective vehicle lengths for density calculations and/or assumed constant queue discharge rates at the bottleneck 
locations, which reduce the accuracy of the estimation (Dhulipala 2002). In addition, using traffic volumes and occupancies to estimate speeds may not be appropriate for uncongested conditions since the speed is not sensitive to traffic demand under these conditions (Vanajakshi 2004). Statistics-based methods such as the probabilistic regression are proven to be robust during congested conditions (Guo 2006). However, such methods usually use a data sampling interval of 1-second, which is not commonly available in practice, where traffic detector data are commonly polled at 20-second or 30-second frequencies in current traffic management applications.

Since different methods perform differently under various traffic conditions, instead of using one simple method, researchers are exploring the development of hybrid approaches to estimate freeway travel time. Vanajakshi (2004, 2009) applied an extrapolation method for very low volume conditions and a traffic flow approach for the remaining situations. The cut-off threshold used to switch between these two methods is $500 \mathrm{veh} / \mathrm{hr} / \mathrm{lane}$, which is selected based on the testing results and seems to be a very low value. Further, using the volume as the threshold may not be appropriate as a given flow rate may correspond to two different conditions: one is an uncongested condition and the other is a congested condition. In addition, this study only investigated the travel time estimation along a very short distance (about 1.9 miles).

Dhulipala (2002) used the shock wave method to estimate travel times under lane-closure and incident conditions, and a Mid-Point method based on flow and density (instead of speed) for non-incident and non-closure conditions. When using the Mid-Point method, the estimates were increased by $20 \%$ when the density at the downstream detector was greater than $60 \mathrm{veh} / \mathrm{mile} / \mathrm{lane}$ and the density at the upstream 
detector was less than 60 veh/mile/lane, for the purpose of capturing the queue propagation. Similarly, a factor of $40 \%$ was applied to the results of the Mid-Point method when both the densities at the upstream and downstream detectors were greater than $60 \mathrm{veh} / \mathrm{mile} / \mathrm{lane}$. These two factors were approximated from the relationship between speed and density measurements. In another study, Xia and Chen (2007) first estimated travel time based on traffic parameters such as flow rate and occupancy measurements, and then adjusted the segment travel time using the shock wave method for incidents with significant impacts. However, this method requires incident starting time and estimates of the initial queue length based on detector measurements.

\subsection{Travel Time Prediction}

Although this study mainly focuses on travel time estimation, it is helpful to review the prior research on travel time prediction. As mentioned in the previous section, travel time prediction forecasts the future travel times. Depending on whether the prediction horizon is less than or greater than 60 minutes, travel time prediction can be classified as either short-term or long-term predictions (Van Lint 2004). Short-term travel time prediction is usually performed based on the near-past or current traffic conditions. In comparison, long-term prediction relies more heavily on assumptions about future traffic conditions. These assumptions are commonly made based on the statistics of historical data. It is logical to expect that the accuracy of travel time prediction decreases as the prediction horizon increases. The review presented below only pertains to short-term freeway travel time prediction as it is more closely related to the travel time estimation investigated in this study. 
Travel time prediction methods include regression analyses, time-series, Kalman Filtering, artificial intelligence, simulations, and more. In regression analysis, some studies assumed the predicted travel time to be a linear function of current and/or past estimated travel time values (Sun et al. 2003; Rice and Zwet 2004), or directly as a function of flow and occupancy measurements from loop detector data (Kwon et al. 2000). The coefficients in these regression expressions were assumed to vary with the departure times in studies by Zhang and Rice (2001) and Kwon and Petty (2005). It was pointed out that these regression models may not perform well under incidents and other abnormal conditions (Waller et al. 2007).

In parallel, research efforts have also been devoted to time series analyses for travel time prediction. D’Angelo et al. (1999) proposed a non-linear time series method for travel time forecasting. Ishak and Al-Deek (2002) applied the same method for travel time prediction on Interstate-4 in Orlando, Florida. The results showed high prediction errors during congested conditions. Guin (2006) applied the Seasonal Autoregressive Integrated Moving Average (SARIMA) Time Series Model to predict travel time. Waller et al. (2007) first used the ARIMA method to forecast traffic counts, which in turn was incorporated in the cumulative method to calculate travel time.

When either the measured travel time data or historical data is available, Kalman filtering is a promising method for travel time forecasting. Chien et al. (2003) considered the current travel time as the state variable, which is a linear function of travel time in previous time intervals. This state variable was updated based on the new observations of travel time obtained from the simulation. Similarly, Vanajakshi et al. (2008) assumed that the subsection travel time is a function of the upstream subsection 
travel time, which is further combined with real time data from GPS-equipped buses to predict the travel time in India.

More recently, various data-based artificial intelligence methods were widely used for travel time prediction. The studies of You and Kim (2000), Sun et al. (2003), and Bajwa et al. (2005) used the nearest neighbor search method to obtain the predicted travel time by finding the closest matching traffic conditions based on the historical data. Wu et al. (2004) predicted the travel time by using the Support Vector Regression (SVR) method. A similar method was also used by Lam and Toan (2008), which improves upon the earlier approach by using an empirical nearest neighbor method to retrieve the closest pattern and therefore substantially reduce the size of the training set of SVR. The results indicated that the SVR method has a better performance than both the historical mean predictor and the time-varying coefficient linear regression model.

Also, among the different kinds of artificial intelligence methods, neural network is quickly becoming one of the most attractive approaches to travel time prediction. The structure of neural networks evolves from simple multilayer feed-forward structures (Park and Rilett 1999; Kisgyörgy and Rilett 2002; Huisken and van Berkum 2003; Innamaa 2005; Tao et al. 2006), and spectral basis (Rilett and Park 1999; Park et al. 1999) to more sophisticated state-space (Van Lint et al. 2002; Van Lint et al. 2005; Van Lint 2006), counter propagation neural (CPN) (Dharia and Adeli 2003), multilayer feedback (Savran 2007), and genetically-optimized time-delay models (Abdulhai et al. 1999). The neural network approach can be further improved by incorporating hybrid preprocessing methods to identify traffic patterns and simplify the inputs, such as the use of Kohonen Self-Organizing Feature Maps (SOFM) ((Park and Rilett 1998; Kisgyörgy 
and Rilett 2002), fuzzy c-means clustering (Park and Rilett 1998), Empirical Mode Decomposition Method (Hamad and Faghri 2005), and grey time series model (Wen et al. 2005). Since there is no single type of neural network that can outperform the others under all prediction horizons, three types of dynamic neural networks were combined together by Ishak et al. (2003; 2004), and the specific type of neural work used for prediction under certain traffic conditions was selected such that the best performance can be achieved. The neural network method acts as a black box, which does not require the knowledge of how the traffic process evolves and is easy to implement. It does require, however, the presence of future traffic conditions in the training network to obtain better performance. Additionally, the trained neural network is not transferable because the neural network input and model selection are always location-specific (Van Lint et al. 2005).

In addition to the above mentioned methods, simulation is also a useful tool for travel time forecasting. Wunderlich et al. (2000) proposed the application of the mesoscopic INTEGRATION simulation model to predict the link travel times for decentralized route guidance. Ben-Akiva et al. (2001) applied the DynaMIT, a dynamic traffic assignment/simulation tool, to estimate network status and predict travel time. Miska et al. (2005) presented a cellular automata model-based real-time microscopic simulation system to predict travel time. Under this model, the prediction horizon can be as large as 30 minutes. To overcome the limitation of sparsely distributed detector data, Liu et al. (2006) developed an on-line travel time prediction framework based on the microscopic simulation software, CORSIM. Waller et al. (2007) later developed an integrated simulation and statistical model based travel time prediction framework. The 
framework uses the time-series method to predict the future traffic count and in turn input these volume counts to the Cell Transmission Model to simulate travel time. The testing results indicated that travel time prediction errors range between $10 \%$ and $23 \%$ for a short three-mile segment with different levels of demand load. The simulation-based travel time prediction approach takes speed limit, geometry conditions, different facility type, traffic signals, and other control strategies into consideration, however, it requires a lot of effort to calibrate this model.

Since each method has its strengths and weaknesses, recently, several studies proposed to combine the different travel time prediction methods to improve overall performance. Li (2002) used wavelet neural network to nonlinearly combine the forecasting results of the artificial neural network and Kalman Filtering models. Kuchipudi and Chien (2003) developed path-based and link-based travel time prediction models by using the Kalman Filtering method, and selected the best results for a specified time based on the prediction errors of each model. Zheng et al. (2003) linearly combined the predictions of back propagation neural network and radial basis function neural network by assigning certain weights, determined by Bayes's rule according to the cumulative performance of each single neural network model. Similarly, Van Hinsbergen and Van Lint (2008) proposed the application of the Bayesian method to combine the prediction models based on their errors under similar conditions during the calibration process. In all of these studies, the results indicated that the hybrid models outperform the single models. Instead of using a single source of data, the data from different sources are also fused together to improve the prediction accuracy. Sun et al. 
(2008) employed the fuzzy logic method to fuse the travel time data obtained from the floating car technique as well as vehicle detector data.

\subsection{Factors Influencing Travel Time Estimation}

Accuracy has been widely used as an important performance measure for travel time estimation, determining how close the estimated travel time is to the actual travel time. Many factors can contribute to the accuracy of travel time estimation, such as detector failures, detector spacing, segment lengths, data aggregation level, travel time estimation algorithms, incidents, weather, and population characteristics. The first four factors in the above list determine the quality of the input data for travel time estimation, which in turn affects the estimation accuracy. The remaining factors directly affect the performance of the estimation process. Many researchers focus on the improvements in travel time estimation algorithms to increase the travel time estimation accuracy. However, few studies have investigated the impacts of other factors on the accuracy of travel time estimates.

Ishak and Al-Deek (2002) developed a general linear model (GLM) to explore the relationship between a congestion index, prediction horizon, rolling step, interaction terms and prediction errors, which showed that these factors have significant impacts on the prediction accuracy. Oh and Park (2008) applied the entropy concept to quantify the impacts of traffic characteristics and found a linear relationship between these characteristics and travel time prediction accuracy. Shen (2008) investigated the impacts of the rolling step on the accuracy of travel time estimation. The study also 
found that the estimation results do not show a significant difference between different data aggregation levels.

Several projects studied the optimal placement of point detectors. More detectors may increase the accuracy of travel time estimation. However, it also results in higher maintenance requirements and thus higher costs. Waller et al. (2007) enumerated the possible detector deployment patterns, and used a cell transmission model-based travel time prediction framework to evaluate the detector deployments. The results highlighted the importance of deploying the detectors close to the merging and diverging sections. Edara et al. (2008) developed a Genetic Algorithm (GA)-based optimization method to determine the optimal locations of detectors, where the objective function is to minimize the errors between the estimated travel time and the actual travel time collected by probe vehicles equipped with GPS. The study results indicated that the existing detector configuration (with a spacing of 0.5 miles) can be reduced by $45 \%$ and still satisfy the minimum travel time error rate. A similar optimization method was also applied by Viti et al. (2008) to determine the optimal sensor locations. Bertini and Lovell (2008) proposed another approach to find the optimal detector spacing, in which they compared the estimated vehicle-hour traveled during the transition condition based on the Mid-Point algorithm with the actual vehicle-hour traveled obtained by using shock wave theory, and determined the optimal detector spacing by minimizing such deviations. Another study was conducted by Kothuri et al. (2008) to understand and reduce real-time travel time estimation errors. In their study, the estimated travel time in Portland, Oregon, was compared with the actual travel time, which showed that the estimated travel time has an error rate of below $20 \%$ for $85 \%$ of the time. Three sources of errors 
were further identified: transition traffic conditions, detector failure, and detector spacing. In addition to the above work, simulation was also performed by these researchers to investigate the impact of additional detectors. The simulation results revealed that the high-density of detectors can improve the accuracy of travel time estimates for most cases but the effects of detector spacing still vary depending on specific locations. Although possible errors are identified by Kothuri et al. (2008), the impacts of these factors on the estimation accuracy have not been extensively investigated.

In addition to accuracy, reliability is also an important performance measure for travel time estimation. For real-world applications, instead of posting one travel time estimate, travel time range is usually displayed on DMS. The reliability of travel time estimation, considering the uncertainty of travel time estimates has not been widely investigated. Van Lint (2004) identified three sources of uncertainty in predicted travel time. These factors are the distribution of individual vehicle travel times, the application of off-line travel time estimation, and the errors in the parameters of State-space neural network (SSNN). This study assumed a lognormal distribution for simulated vehicle travel time and normal distribution for real-world vehicle travel time, estimated travel time, and errors associated with neural network prediction. The resulted Prediction Interval Coverage Percentage (PI_CP), defined as the number of observations that fall in the confidence interval, was employed to represent the performance measures for the uncertainty of travel time prediction.

$\mathrm{Li}$, Rose, and Sarvi (2007) proposed a simple approach to generate the bounds for estimated travel time. The approach is to use the minimum lane speeds at both upstream and downstream detector stations to get the upper bound of travel time estimation, and 
similarly use the maximum lane speeds at both locations for the lower bound. The assumption behind this approach is that fast drivers always tend to drive fast. Gaudio (2008) investigated the effects of segment length and traffic congestion level on the reliability of travel time estimation by comparing the dynamic estimated travel time with those posted on DMS. Regression analysis was then conducted to relate traffic volume and segment length to the reliability of travel time estimation. However, this study defined the reliability as the difference between the posted travel time and dynamic estimated travel time.

\subsection{Summary}

In this chapter, a comprehensive review was performed to investigate topics related to travel time measurement, detector data filtering and imputation, existing travel time estimation and prediction methods, and factors influencing estimation accuracy and reliability. Below, a summary of the findings based on the literature review is given.

Previous research has demonstrated the necessity of data preprocessing for the accurate and reliable estimation of travel time. To identify the erroneous detector data, the threshold-based filtering method has been widely used in the literature. Methods

with various degrees of complexity have been applied to replace the missing data. However, the simplest among the existing methods, linear interpolation, also performs well.

Existing speed-based or flow-based travel time estimation methods can only perform well under certain conditions. The hybrid travel time estimation approach has 
been proposed as an effective method to estimate travel time at different congestion levels, but has not been adequately explored and compared with the existing methods.

Although recent studies focused on developing travel time prediction algorithms, when the ground-truth data are not available, the prediction has to rely on estimated travel time inputs, which are usually based on the simple speed-based travel time estimation. An improvement in travel time estimation may thus further improve the travel time prediction.

Travel time estimation performance can be quantified in terms of accuracy and reliability. Although some studies have been conducted to investigate the impacts of certain influential factors on estimation accuracy, studies on estimation reliability and its associated influential factors have been limited. 


\section{CHAPTER 3}

\section{METHODOLOGY}

This research aims at developing improved hybrid models to estimate freeway travel times under different congestion levels based on point detectors. Figure 3-1 presents the framework of the developed methodology. As shown in this figure, the methodology developed in this study includes data acquisition and preprocessing, traffic condition identification, travel time estimation, and the assessment of the estimation quality.

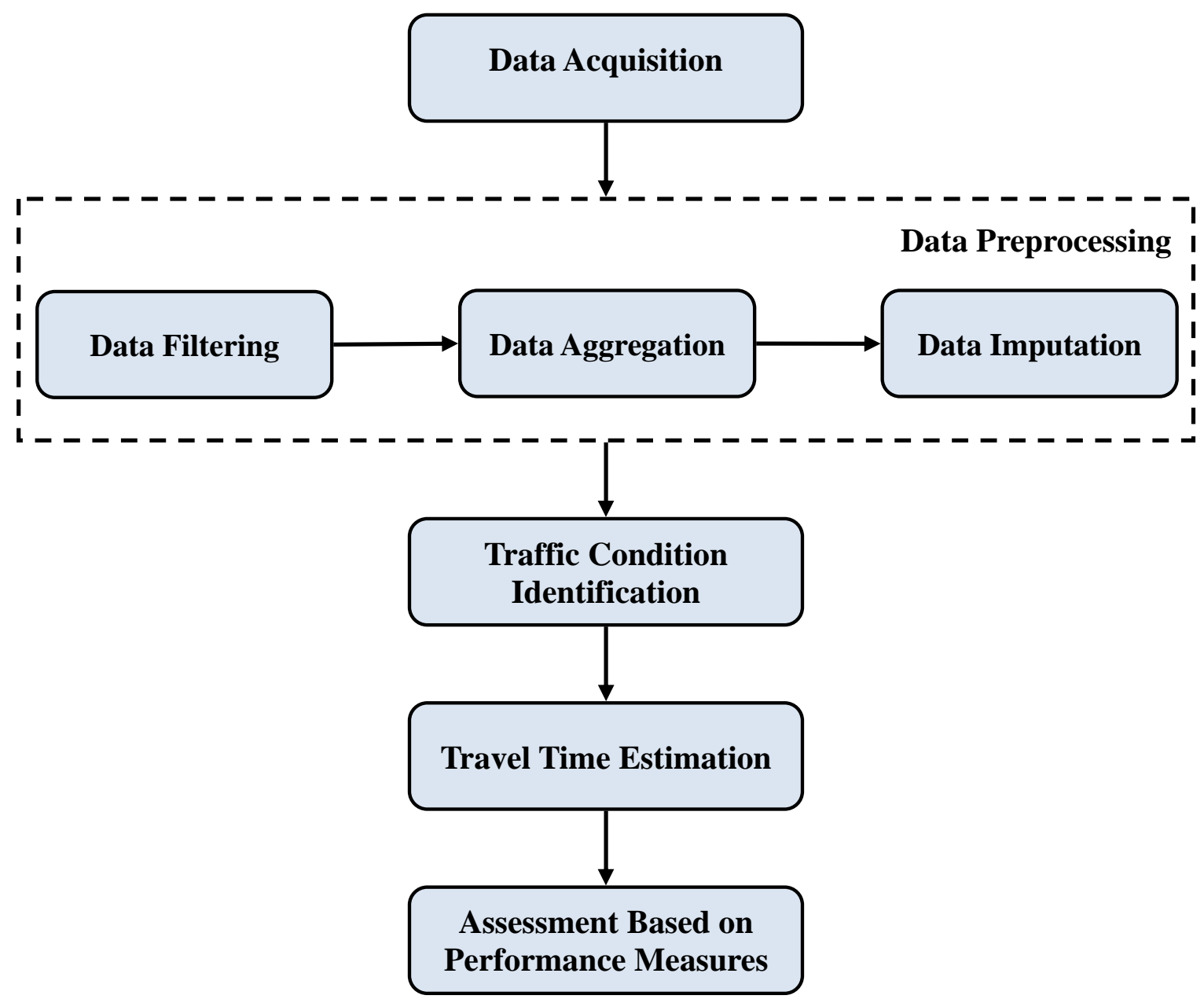

Figure 3-1 Travel Time Estimation Framework 


\subsection{Data Acquisition and Preprocessing}

The traffic data used in this study are collected using point detectors (true-presence microwave detectors) that are deployed along the major freeways in Miami, Florida, as shown in Figure 3-2. These point detectors measure the values of speed, volume, and occupancy at small time intervals (e.g., every 20 seconds). The detector data is then archived by the freeway traffic management software, which in Florida is the SunGuide software, and used by the traffic management centers (TMCs) of the Florida Department of Transportation (FDOT). This data is stored in text files referred to as TSS files that contain one record for each lane of each detection station for every 20-second polling interval, as shown in Figure 3-3.

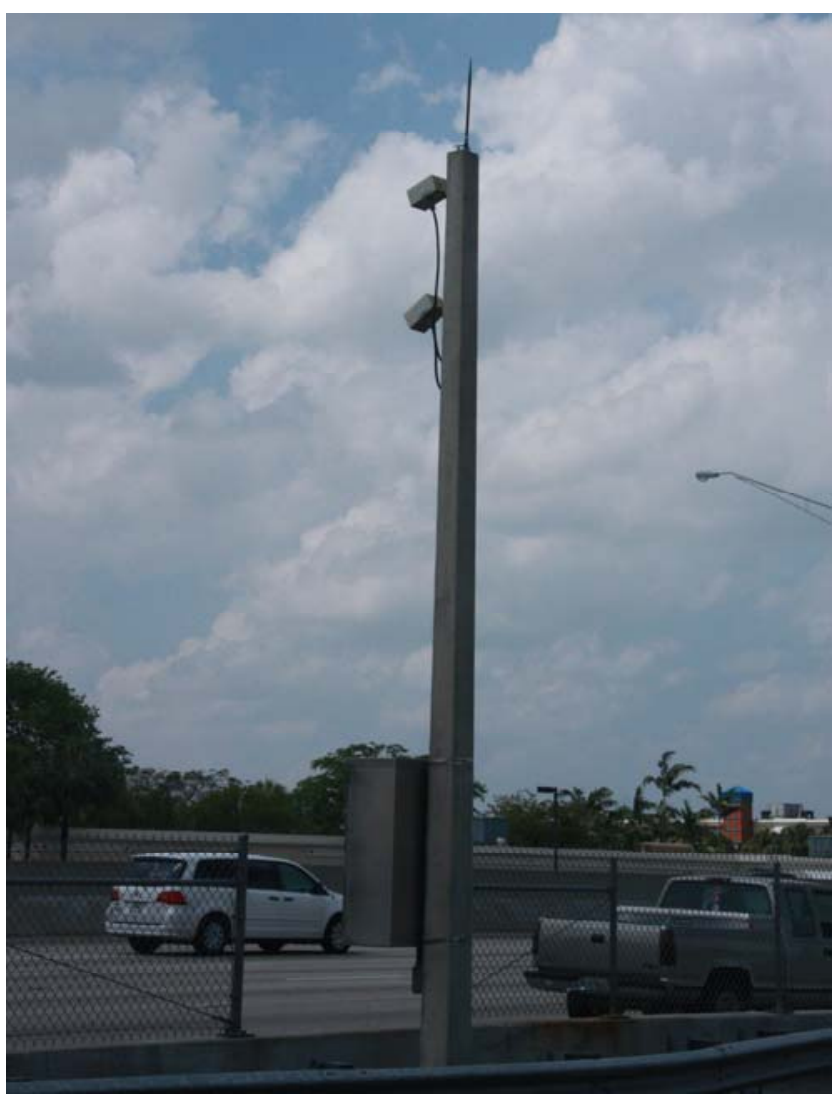

Figure 3-2 Example of Microwave Detectors 


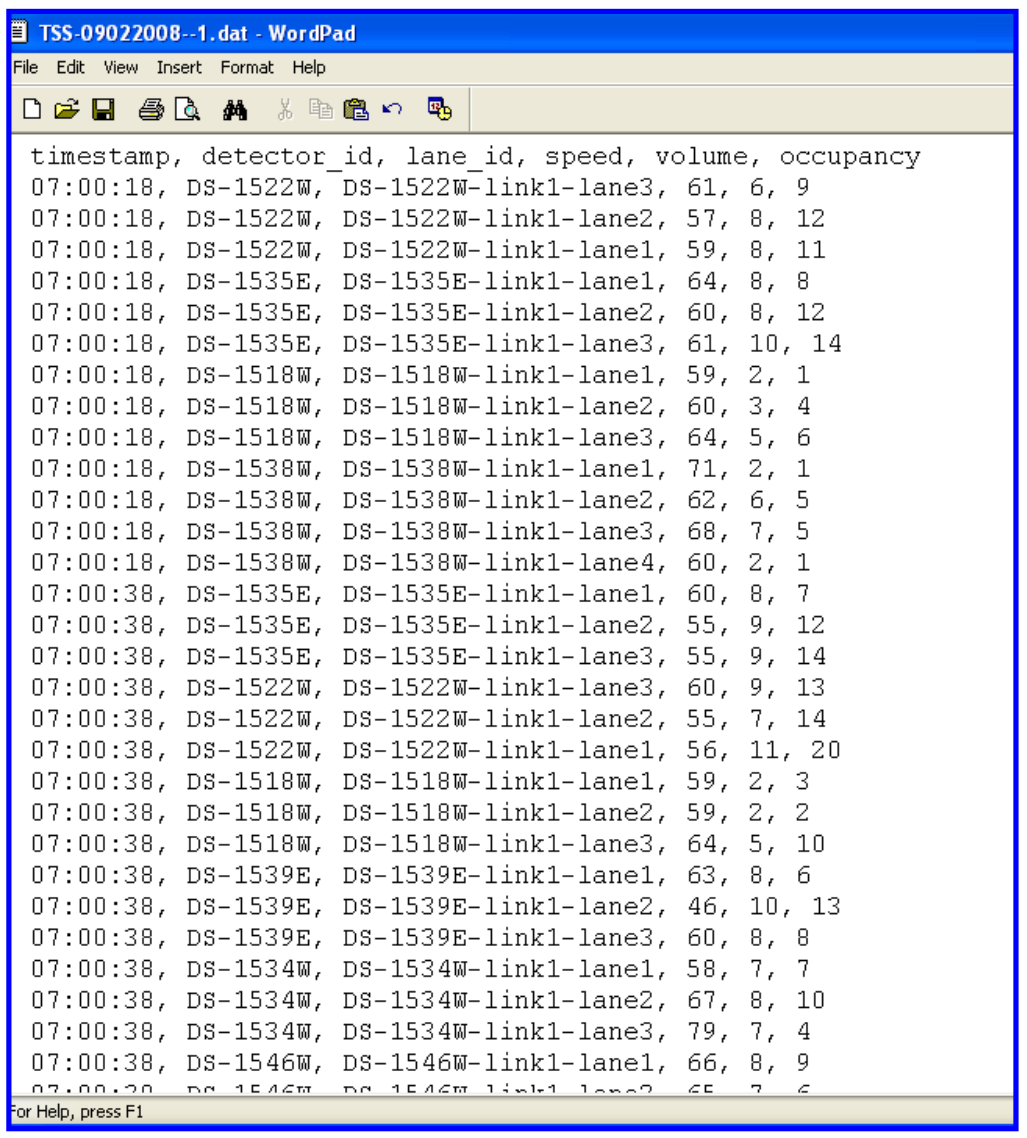

Figure 3-3 Example of Detector Data File

Detector data of a good quality is prerequisite for accurate and reliable travel time estimation. However, current detector data usually include erroneous or missing measurements. As one example, Table 3-1 presents some obvious errors in detector data along the SR-826 East-West section on December 2, 2008. As listed in this table, some errors may exist in the data: for example, duplication of the same records, records with the same timestamp and same detector but with different values, or records with the same values but an elapsed time of less than 20 seconds. In addition, there is a need to optimize the aggregating and smoothing of the measurements for different applications; 
data preprocessing procedures are therefore required. These procedures include data filtering, temporal and spatial aggregation, and data imputation as described below.

Table 3-1 Example of Detector Errors

\begin{tabular}{|c|c|c|c|c|c|}
\hline \multicolumn{6}{|c|}{ Detector Data $(12 / 2 / 2008)$} \\
\hline \multicolumn{6}{|c|}{ Type 1 Error: Number of exactly duplicate records } \\
\hline timestamp & detector_id & lane_id & speed & volume & occupancy \\
\hline 01:06:40 & DS-1533E & DS-1533E-link1-lane2 & 0 & 0 & 0 \\
\hline 01:06:40 & DS-1533E & DS-1533E-link1-lane2 & 0 & 0 & 0 \\
\hline \multicolumn{6}{|c|}{$\begin{array}{l}\text { Type } 2 \text { Error: Number of same timestamp, same detector, but with different } \\
\text { traffic data }\end{array}$} \\
\hline timestamp & detector_id & lane_id & speed & volume & occupancy \\
\hline 11:58:00 & DS-1515E & DS-1515E-link1-lane1 & 48 & 6 & 9 \\
\hline 11:58:00 & DS-1515E & DS-1515E-link1-lane1 & 49 & 1 & 1 \\
\hline \multicolumn{6}{|c|}{ Type 3 Error: Number of same values, but with time interval less than 20 seconds } \\
\hline timestamp & detector_id & lane_id & speed & volume & occupancy \\
\hline 03:42:54 & DS-1523E & DS-1523E-link1-lane2 & 0 & 0 & 0 \\
\hline 03:42:57 & DS-1523E & DS-1523E-link1-lane2 & 0 & 0 & 0 \\
\hline \multicolumn{6}{|c|}{ Type 4 Error: Number of missing data } \\
\hline timestamp & detector_id & lane_id & speed & volume & occupancy \\
\hline 01:30:34 & DS-1523E & DS-1523E-link1-lane2 & 0 & 0 & 0 \\
\hline 03:42:54 & DS-1523E & DS-1523E-link1-lane2 & 0 & 0 & 0 \\
\hline
\end{tabular}

\subsubsection{Data Filtering}

Rule-based tests are applied first to identify erroneous detector data. These rules are set based on an examination of the types of errors commonly found in the detector data archived by the SunGuide software. The rule-based tests include the following steps:

- Identify duplicate data records such as the same records repeated more than once; two records with the same timestamp and lane identification (ID) but with different measurements (speed, volume count, and occupancy values); or data with the same lane ID and same measurements but with a polling interval of less than 20 seconds. 
- A univariate test of data measurements aggregated at the 20-second level to check whether the values of individual traffic parameters exceed predefined minimum or maximum thresholds. As shown in Table 3-2, the minimum and maximum thresholds for speed measurements are zero and $30 \mathrm{mph}$ more than the speed limit; zero and 17 for volume counts, and zero and $100 \%$ for occupancy measurements. These thresholds are based on recommendations by Payne et al. (1976) and Quiroga et al. (2005).

- A multivariate test of data measurements aggregated at the 20-second level to check for unreasonable combinations of traffic parameter values such as a combination of zero speed, zero occupancy, and non-zero volume values. Note that the detector data with zero speed, volume count of one, and occupancy greater than $60 \%$ are considered to be valid for representing extremely congested conditions, as the zero speed may be caused by a rounding error. Similar reasoning is applied for detector data with non-zero speed, zero occupancy, and a volume count of greater than $52.8 \times S /\left(180 \times L_{\text {eff }}\right)$.

This volume threshold is based on the relationship between occupancy and speed when the occupancy is equal to one (Turner 2007). The average effective vehicle length, $L_{\text {eff, }}$ defined as the summation of average vehicle length and detector data, is assumed to be $25 \mathrm{ft}$ in this step. The coefficient of 180 in the above expression is used to convert the hourly volume to the data at the 20-second level to be consistent with the data reported by the detector. Detector data with zero speed, zero volume count, and an 
occupancy that is less than $3 \%$ is considered to be acceptable since this may occur when a vehicle positioned at the start of the new 20-second period is above a detector (Jain and Coifman 2005). Detector data of zero speed, zero volume count, and $100 \%$ occupancy is also valid as vehicles stop on the detector when there is an incident or traffic is extremely congested.

- A temporal variability check to test for constant values of speed, volume, and occupancy for a long period of time, including all zeros. The threshold for number of periods beyond which having constant values should be flagged for errors varies with the time-of-day and locations, as discussed by Turner (2007) and University of Florida Transportation Research Center (2005). Since detector data of zero speed, zero volume count, and zero occupancy can correspond to no traffic conditions, this type of data is not filtered out. However, cases in which all zero values are consistently reported are flagged for error in this step.

- Multivariate tests for the average effective vehicle length and maximum density at the temporal aggregation level under consideration. Based on traffic flow theory, density can be calculated from flow and speed, which in turn can be combined with occupancy to estimate the average effective vehicle length. Since the average effective vehicle length and maximum density are not linear functions of speed, volume, and occupancy, the combination of these three parameters may yield the reasonable average effective vehicle length and density values at the 20-second level, but may still produce invalid values when detector data is aggregated to the level of 
1-minute or more. Therefore, this test is conducted at the aggregated data level instead of the 20-second raw data level. In this study, the reasonable range of average effective vehicle length is assumed to be between 10 and 60 $\mathrm{ft}$, and the maximum allowable density is $250 \mathrm{vphpl}$.

It should be pointed out that a temporal consistency check, which is a check for the degree of change in the measured values for two consecutive time periods, is not conducted because it may produce incorrect conclusions. As one example, detector may detect vehicles during the first 20 seconds, but no vehicle for the next 20 seconds due to light traffic. Also, the corresponding speed can be reduced from free-flow speed to zero. Another example is when a vehicle at the end of a queue stops on the detector for the first 20 seconds and moves away from the detector at the start of the next 20 seconds. The possible ranges of measurement changes between two consecutive periods are -100 100 mph for speed, $-17 \sim 17$ for volume count, and $-60 \sim 60$ for occupancy measurements for two consecutive 20-second periods, which are very large and almost the same as the thresholds set for individual values. Therefore, the temporal consistency check is not conducted since it is not expected to contribute to detecting errors.

A spatial check for the relative differences in traffic parameters between detectors at neighboring stations is also not conducted, as big differences between lanes at the same location and detectors at neighboring stations may exist during lane closures, off-ramp queue backups, and incident conditions. Further, the difference between two neighboring stations may be due to on-ramp and/or off-ramp volumes. Similar to the temporal consistency check, this criterion can cause legitimate data to be filtered out. Detailed tests based on the above criteria are listed in Table 3-2. 
Table 3-2 Rule-based Tests Used in the Data Filtering Step

\begin{tabular}{|c|c|c|}
\hline $\begin{array}{l}\text { Aggregation } \\
\text { Level }\end{array}$ & \multicolumn{2}{|l|}{ Tests } \\
\hline \multirow[t]{4}{*}{$\begin{array}{l}\text { 20-second } \\
\text { Detector Data }\end{array}$} & $\begin{array}{l}\text { Eliminate } \\
\text { Duplicate data }\end{array}$ & $\begin{array}{l}\text { - Eliminate exactly same records } \\
\text { - Identify data with same timestamp and lane } \\
\text { ID but different speed, volume count, and } \\
\text { occupancy measurements } \\
\text { - Identify data with the same lane ID, speed, } \\
\text { volume count, and occupancy measurements } \\
\text { but with time interval less than } 20 \text { second }\end{array}$ \\
\hline & Univariate Test & $\begin{array}{l}\text { - } S<0 \text { or } S>\text { Speed limit }+30 \text { mph } \\
\text { - } V<0 \text { or } V>17 \text { for } 20 \text {-second data } \\
\text { - } O<0 \text { or } O>100\end{array}$ \\
\hline & $\begin{array}{l}\text { Multivariate } \\
\text { Test }\end{array}$ & $\begin{array}{l}\text { - } S=0, V>0 \text {, and } O>0 \quad \text { (except that } S=0 \text {, } \\
V=1 \text { and } O>=60) \\
\text { - } S>0, V=0 \text {, and } O>0 \\
\text { - } S>0, V>52.8 \times S /\left(180 \times L_{e f f}\right) \text {, and } O=0 \\
\text { - } S=0, V=0 \text {, and } 3<O<100 \\
\text { - } S=0, V>0 \text {, and } O=0 \\
\text { - } S>0, V=0 \text {, and } O=0\end{array}$ \\
\hline & $\begin{array}{l}\text { Temporal } \\
\text { Variability Test }\end{array}$ & $\begin{array}{l}\text { - Check the maximum consecutive periods of } \\
\text { constant speed, volume count, and } \\
\text { occupancy including all zeros } \\
\text { - Maximum of } 30 \text { periods (i.e., } 10 \text { minutes) } \\
\text { for 6:00 am - 10:00 pm } \\
\text { - Maximum of } 45 \text { periods (i.e., } 20 \text { minutes) } \\
\text { for 10:00 pm - 12:00 am } \\
\text { - Maximum of } 90 \text { periods (i.e., } 30 \text { minutes) } \\
\text { for 12:00 am - 6:00 pm }\end{array}$ \\
\hline \multirow[t]{2}{*}{$\begin{array}{l}\text { Temporal } \\
\text { Aggregated } \\
\text { Detector Data }\end{array}$} & $\begin{array}{l}\text { Average } \\
\text { Effective } \\
\text { Vehicle Length }\end{array}$ & $\begin{array}{l}\text { - } L_{\text {eff }}=52.8 \times O \times S / V \\
\text { - } L_{\text {eff }}<10 \mathrm{ft} \text { or } L_{\text {eff }}>60 \mathrm{ft}\end{array}$ \\
\hline & $\begin{array}{l}\text { Maximum } \\
\text { Density }\end{array}$ & $\begin{array}{l}\text { - } k=V / S \\
\text { - } k>250 \text { vphpl }\end{array}$ \\
\hline
\end{tabular}

Note: $S, V, O$ represent speed, volume counts, and occupancy measurements, respectively. 


\subsubsection{Data Smoothing and Aggregation}

The filtered 20-second lane-by-lane detector data must be smoothed to reduce the impacts of noise in the detection data. Two smoothing methods are tested in this study: the simple moving average method used in the SunGuide software and the exponential moving average method. The simple moving average is the average of previous $m$ data points, where $m$ is specified by the rolling period. The mathematical expression for the simple moving average method is the following:

$$
Y_{t}=\frac{1}{m} \sum_{i=1}^{m} X_{i}
$$

where $X_{i}$ represents the raw $i^{\text {th }}$ measurement while $Y_{t}$ is the smoothed traffic parameter at the $t$ timestamp.

The second type of smoothing, the exponential moving average method, is described in Equation 3-2.

$$
Y_{t}=\alpha X_{t}+(1-\alpha) Y_{t-1}
$$

The symbol $\alpha$ in Equation 3-2 refers to a smoothing factor selected by the user. Since detectors may have incidental or structural failures, the time interval between two acceptable neighboring records may not be consistent, and therefore a time-dependent smoothing factor is used instead of a constant value. The expression for $\alpha$ is shown in Equation 3-3 below:

$$
\alpha=1-e^{-\Delta t / \tau}
$$

where $\Delta \tau$ is the time interval between two consecutive records. $\tau$ is a time constant, estimated based on a time interval of 20 seconds between detector data measurements and the commonly used value of 0.4 for $\alpha$ (Shen 2008). 
It should be mentioned that zero speed measurements are not included in the smoothing when both volume count and occupancy measurements are zeros, since this corresponds to a no vehicle condition. Depending on the frequency of updating travel time estimation, the most recent smoothed lane data is further aggregated to the station level for use in a later step. Below is the expression for spatial aggregation:

$$
\left\{\begin{array}{l}
S_{s}=\frac{S_{l, 1}+S_{l, 2}+. .+S_{l, N}}{N} \\
V_{s}=V_{l, 1}+V_{l, 2}+\ldots+V_{l, N} \\
O_{s}=\frac{O_{l, 1}+O_{l, 2}+\ldots+O_{l, N}}{N}
\end{array}\right.
$$

where the speed $S_{s}$ and occupancy $O_{s}$ are the averages of lane speeds and lane occupancies, respectively, at station $s$, and the volume count at the station, $V_{s}$, is the summation of all lane counts. The parameter $N$ denotes the total number of lanes. Similar to data smoothing, when the lane-based detector data are all zeroes, zero speed is not considered in the calculation of station speed, since detector data for all zeroes indicates that no vehicle passes the detector during the aggregated period of time.

\subsubsection{Data Imputation}

In the SunGuide software, the measured lane-based speed data is smoothed by a simple moving average method, then capped by the speed limit to avoid the reporting of short travel times which may encourage speeding. The lane-based data is further aggregated to the station-level and used for travel time estimation. In cases of detector failures, when a speed measurement is not reported by the detection system, the missing speed data is replaced by neighboring data (Dellenback and Heller 2006). This method first 
attempts to use a neighboring lane measurement and, if this is not available, uses a neighboring station measurement.

In this study, additional spatial and temporal data imputation procedures are investigated to substitute for the missing or erroneous data identified in the data filtering step. The spatial imputation employs neighboring detector information to impute the missing values while the temporal imputation uses the past information from the same detector to replace the missing values. In this study, the spatial data imputation procedure is conducted at two levels: within the same detector station and between stations. For the same detector station, if only part of the lanes have missing or erroneous speed measurements, the missing values are filled with the average of the speeds measured by detectors on adjacent lanes with the available speed measurements. When the whole station data is missing, between stations the imputation approach is used. Three types of methods for between-station imputations were considered in this study: simple average, linear interpolation, and the factor method.

The simple average uses the average of measurements from neighboring stations to estimate the missing values, as shown in Equation 3-5:

$$
Y_{2, t}=\frac{1}{2}\left(Y_{1, t}+Y_{3, t}\right)
$$

where $Y$ can be speed, volume count, or occupancy. $Y_{2, t}$ is the estimated value for missing data at timestamp t. $\quad Y_{1, t}$ and $Y_{3, t}$ are the measurements at neighboring stations, as shown in Figure 3-4. 


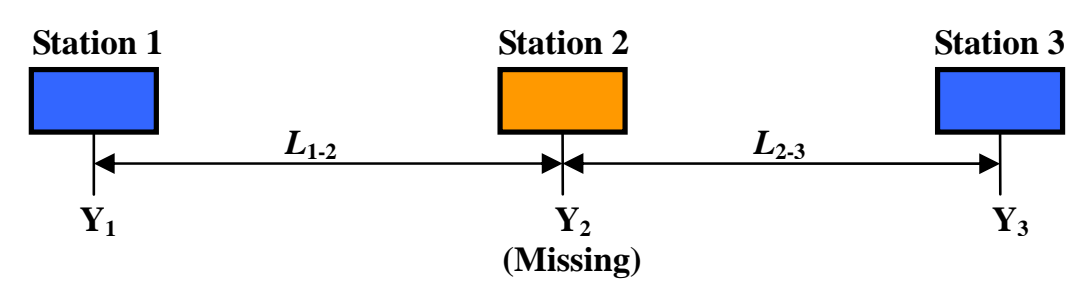

Figure 3-4 Sketch Diagram for Spatial Imputation

The linear interpolation method assumes linear spatial variation of traffic parameters. Thus, the missing data can be estimated as:

$$
Y_{2, t}=\frac{L_{2-3}}{L_{1-2}+L_{2-3}} Y_{1, t}+\frac{L_{1-2}}{L_{1-2}+L_{2-3}} Y_{3, t}
$$

where $L$ denotes the distance between neighboring stations. For the factor method, the factor is defined as the ratio of current station traffic data to the upstream or downstream values, as described below:

$$
F_{i, j, t}=\frac{1}{p} \sum_{p} \frac{Y_{i, t}}{Y_{j, t}}
$$

where $F_{i, j, t}$ represents a factor between station $i$ data and its neighboring station $j$ at time of day $t$. The station $j$ can be either the upstream station or downstream station. $\quad p$ is the total number of days available. The missing value is then estimated as:

$$
Y_{2, t}=\frac{F_{2,1, t} Y_{1, t}+F_{2,3, t} Y_{3, t}}{2}
$$

In this study, the exponential moving average method is applied for temporal imputation. Interpolation could not be used as the future detector data are not available for on-line applications. The missing value is replaced by the forecasted value based on the exponential moving average. The corresponding expression is as follows:

$$
Y_{2, t}=f_{2, t}=\alpha Y_{2, t-1}+(1-\alpha) f_{2, t-1}
$$


where $f$ represents the forecasted values. Note that the formulation in Equation 3-9 is similar to that in Equation 3-2. However, the values in the previous time period are used to impute the missing data at the current time period. Based on previous studies by Van Lint (2005) and Shen (2008), the value of the smoothing factor $\alpha$ is set to 0.4.

In addition, if some required data for the estimation is not measured by the detector system, such as the ramp volume, the historical average at the same time period on same day of the week or short term counts are applied to replace the missing value.

\subsection{Congestion Level Identification}

The hybrid approaches investigated in this study to estimate travel time use combinations of methods and switch between these methods depending on the congestion levels. A key point for the success of these approaches is to identify the roadway conditions and determine the time at which traffic congestion occurs. Furthermore, in the case of traffic congestion that involves queuing, the methodology requires the determination of whether the traffic queue is forming or dissipating. In addition to its use in travel time analysis, the identification of congestion level is important for situation analysis and performance measurements.

Previous studies used predefined speed thresholds (for example, $35 \mathrm{mph}$ or 50 mph) or predefined occupancy thresholds to identify the congestion (Chan 2003; Ban and Benouar 2007; Yeon and Ko 2007; Zhang and Levinson 2004). Kaneko et al. (1995) recommended using all three traffic parameters: speed, volume, and occupancy to identify traffic status. In this study, the k-means clustering algorithm is used to classify the traffic states at each detection station into different clusters based on the historical 
measurements of all three traffic parameters. The reason to select k-means clustering method instead of hierarchical clustering methods is due to the simplicity and computational efficiency of the k-means method. In the clustering analysis, the measurements are normalized using the z-score method to account for the different scales in three traffic parameters, as shown in Equation 3-10.

$$
Y^{\prime}=\frac{Y-Y_{\text {mean }}}{\sigma_{Y}}
$$

where $Y$ refers to any measurement of speed, flow rate, and occupancy, and $Y$ ' is the normalized value. $Y_{\text {mean }}$ is the mean of each type of measurements, while $\sigma_{Y}$ is the corresponding standard deviation. The Euclidean Distance is used to quantify the dissimilarity between two data points:

$$
d_{i j}=\sqrt{\left(S_{i}^{\prime}-S^{\prime}{ }_{j}\right)^{2}+\left(q_{i}^{\prime}-q^{\prime}{ }_{j}\right)^{2}+\left(O_{i}^{\prime}-O^{\prime}{ }_{j}\right)^{2}}
$$

where $d_{i j}$ is the Euclidean Distance between data point $i$ and $j$. S', q,' $O^{\prime}$ are the normalized speed, flow rate, and occupancy, respectively.

The selection of number of clusters $k$ is critical to the success of $k$-means clustering analysis. The classic traffic flow theory divides all the traffic states into two phases: uncongested and congested. However, the study of Hall et al. (1992) argued that the uncongested conditions can be divided into two subphases. The speed within the first subphase is almost constant while the speed in the second subphase decreases. Kerner and Rehborn (1996) further categorized the congested freeway into synchronized flow and wide-moving jams, and thus developed a traffic theory that distinguishes between three phases: free-flow, synchronized flow, and wide-moving jams. Under the free-flow conditions, drivers can drive at their own speed. However, drivers within the 
synchronized flow drive at reduced speeds in this phase, and the flow is still high, while both speed and flow within the wide-moving jams are low. Based on these reviews, the number of clusters used in this study is four. These four clusters are associated with different congestion levels in the fundamental traffic flow theory diagram. When applying the proposed method to estimate travel times in real-time, traffic measures at each detection station are associated with one of the predetermined clusters (congestion levels) for the detection station based on its distances from each cluster centroid, as reflected by the Euclidean Distance. To reduce the impacts of the initial centroid position selections, the clustering analysis is replicated 10 times, and the one with the lowest within-cluster sums of point-to-centroid distances is used as the final result.

Figure 3-5 presents an example of the clustering results for a detector station, DS-1507E, located on SR-826 eastbound in Miami-Dade County, Florida, based on the traffic detector data from December 1, 2008 to December 31, 2008. The four cross symbols in these figures denotes the locations of cluster centroids. As shown in Figure 3-5, the traffic states are separated into four clusters. Cluster I corresponds to close to free-flow conditions and the average speed is almost constant at free flow speed regardless of the demand. Traffic Cluster II is still uncongested but with a reduced speed. Cluster III is a more congested region, where the speed drops but to a lesser degree than the points in Cluster IV. Cluster IV corresponds to extremely congested conditions, with low speed and low constrained flows. Four clusters were used instead of just two corresponding to the congested and uncongested regions, to allow more flexibility in combining or separating any two of the clusters based on the results of the analysis. 


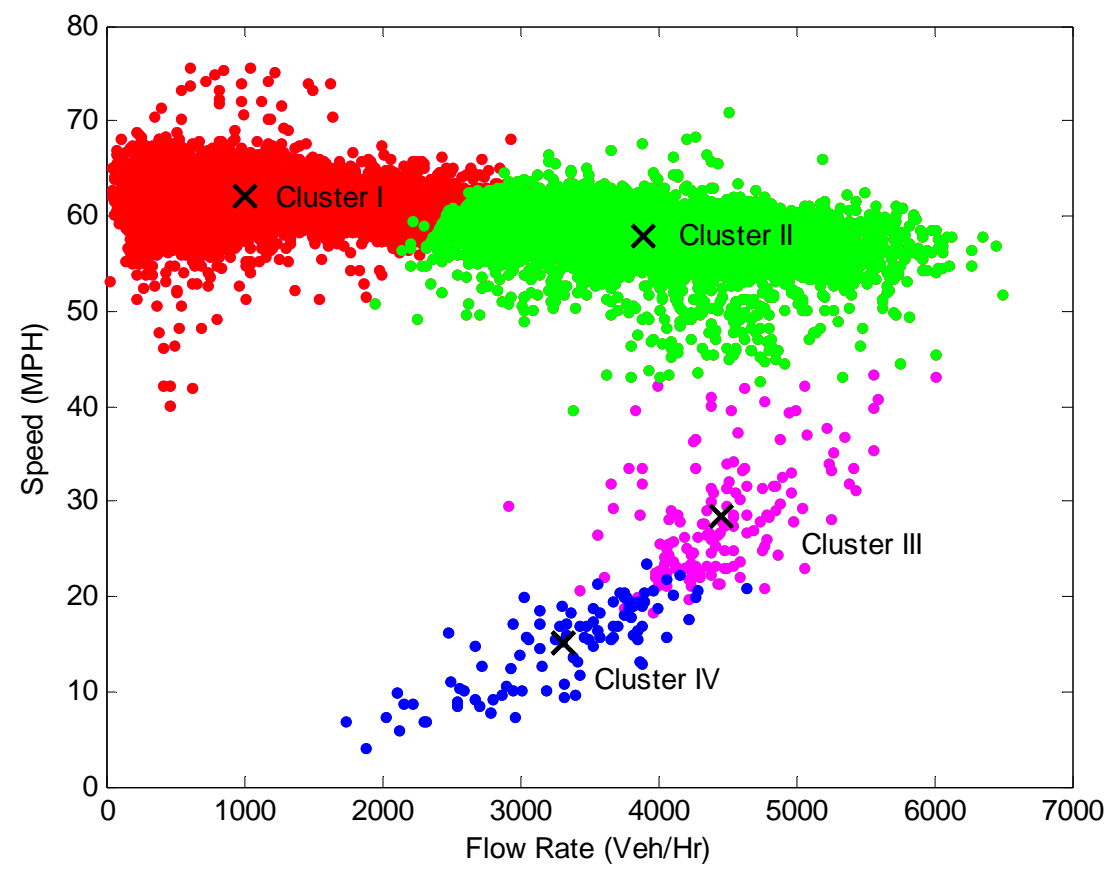

(a) Flow Rate vs. Speed

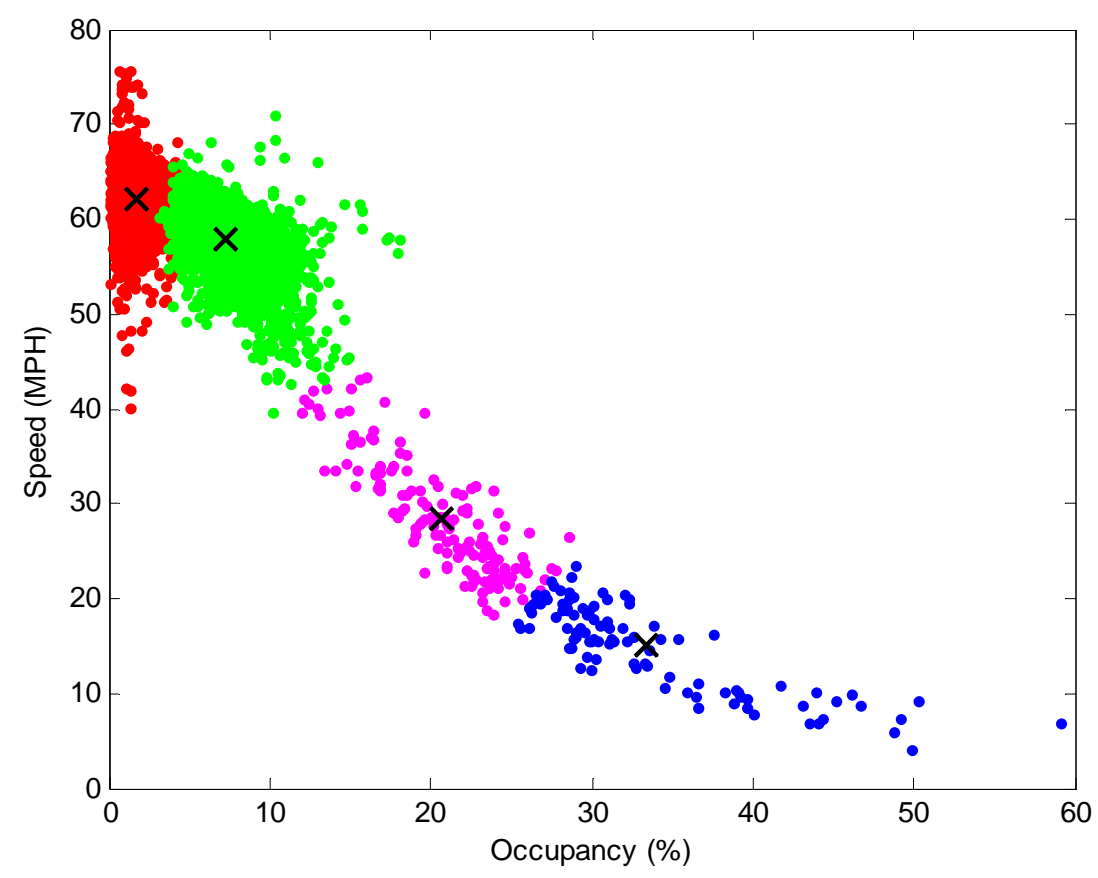

(b) Occupancy vs. Speed

Figure 3-5 Clustering Results for a Detection Station on SR-826

(continued on next page) 


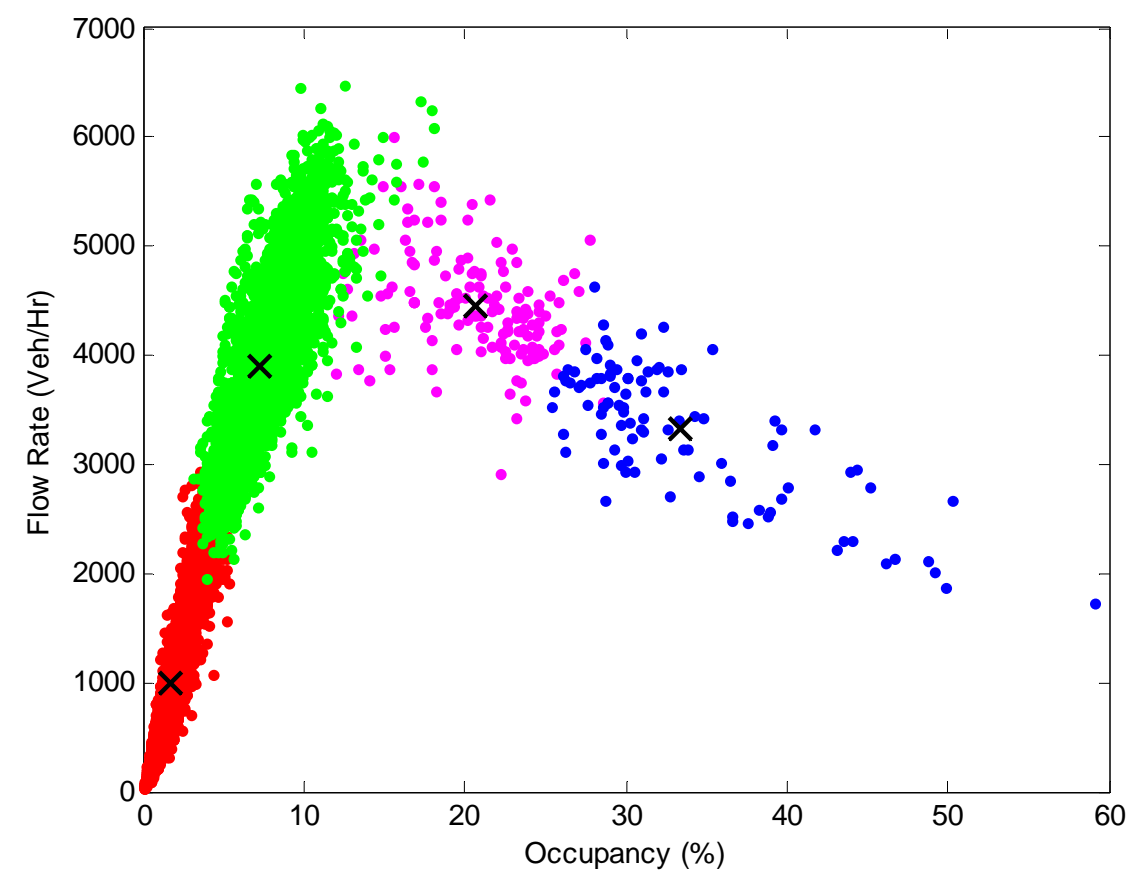

(C) Occupancy vs. Flow Rate

Figure 3-5 Clustering Results for a Detection Station on SR-826

\subsection{Queue Length Estimation}

This study requires the estimation of queue length when using the travel time estimation method based on traffic flow theory. This section discusses how the queue length is estimated in this study based on detector data.

Based on the identified traffic congestion states at the upstream and downstream detector stations in the previous step, each roadway link can be identified as one of four states: head of a queue, tail of a queue, in queue, or outside a queue. For an identified head of a queue link, the traffic state at the upstream station is determined to be congested, that is, in Cluster III or IV, and the traffic states at the first and second downstream stations are in Cluster I or II, as shown in Figure 3-6(a). The consideration of the second downstream station in addition to the first is to avoid a situation where a 
temporary traffic state change in the first downstream station can result in a sudden change in the calculated queue length value. On the other hand, if both the two upstream stations are uncongested, that is, in the region of Cluster I or II, and the downstream station is in the Cluster III or IV, the link is categorized as a tail of a queue, as shown in Figure 3-6(b). The links between a head of a queue link and a tail of a queue link are identified as in-queue links, and the remaining links are identified as outside of queue links.

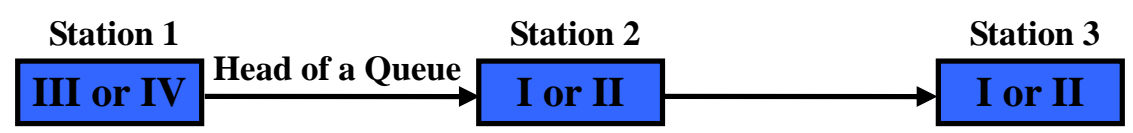

(a) Head of a Queue

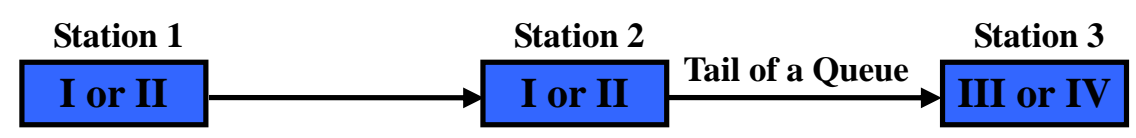

(b) Tail of a Queue

\section{Figure 3-6 Identification of Head and Tail of a Queue}

(Note: the number shown in the box represents the state)

Once the head and the tail of a given queue are identified, the queue length can be estimated. In addition, as another important parameter to the methodology, the queue status (growing, dissipating, or stationary), can be determined by comparing the current locations of the head and tail of the queue with those at previous timestamps. Figure 3-7 presents one example of queue identification for the SR-826 limited access facility in the eastbound direction on January 13, 2009. As shown in this figure, the queue starts from the link located between station DS-1533E and DS-1535E at time 7:08 A.M. and extends to the first upstream link due to an incident at DS-1533E. The queue length starts to decrease at time 8:16 A.M. and completely dissipates at 8:48 A.M. 

$0 \quad$ Uncogested
1 Head of queue
2 Tail of queue

3 Minutes)

Figure 3-7 Example of Queue Identification Results 


\subsection{On-Line Travel Time Estimation}

Travel time is estimated in this study using different methods for on-line (based on real-time data) and off-line (based on historical data) applications. As clarified later in this chapter, additional information is available in the case of off-line applications, allowing for the more accurate estimation of travel time. This section describes the on-line travel time estimation methods investigated in this study.

As mentioned earlier, the hybrid approaches developed in this study apply different methods to estimate travel time based on the identified congestion level and queue location. Two on-line hybrid travel time estimation models are developed herein: the first, referred to as On-Line Hybrid Model 1, combines a speed-based method with a traffic flow theory-based method; the second, referred to as On-Line Hybrid Model 2, combines two different speed-based methods. Below is a description of these hybrid models.

\subsubsection{On-Line Hybrid Model 1}

The rationale behind Model 1 is that previous studies have reported that speed-based travel time estimation methods work well under free-flow conditions, while travel time estimation methods based on traffic flow theory work well under congested conditions. Thus, the combination of these two estimation methods has the potential of producing a better performance. Model 1 uses a speed-based method to estimate travel times for non-congested segments and a traffic flow theory-based method for congested segments.

The speed-based method selected for use is the Mid-Point method, since it is widely used in practice and was shown in this study to perform well for uncongested 
conditions, as described later. This method estimates the travel time along uncongested links as follows:

$$
T T_{i, t}=\frac{L_{i}}{2 S_{i, 1, t}}+\frac{L_{i}}{2 S_{i, 2, t}}
$$

where $T T_{i, t}$ is the estimated travel time for link $i$ at time $t . \quad L_{i}$ denotes the length of the link that connects upstream and downstream detector station, and $S_{i, 1, t}$ and $S_{i, 2, t}$ are the speeds at the detection stations upstream and downstream of the link, respectively.

The travel time for a congested (fully queued) link is estimated using an improved traffic dynamics approach developed by Vanajakshi (2009), as follows:

$$
T T_{i, t}=\frac{L_{i} k_{i, t}}{q_{i, 2, t}}=\frac{L_{i}\left(k_{i, 1, t}+k_{i, 2, t}\right)}{2 q_{i, 2, t}}
$$

where $q_{i, 2, t}$ is the flow rate at downstream station of the link $i$ at time $t$. The $k_{i}$ in this equation is the link density, which is calculated as the average of the densities at the immediate upstream and downstream stations. It should be mentioned that the density $k$ in the above equation is estimated from the measured occupancy and the average effective vehicle length estimated by using the measured values of speed, volume count, and occupancy when the traffic is free-flow, that is,

$$
\begin{aligned}
& L_{e f f, j}=\frac{1}{m} \sum_{t} \frac{52.8 \times O_{i, j, t} \times N_{j} \times S_{i, j, t}}{q_{i, j, t}} \\
& k_{i, j, t}=\frac{52.8 \times O_{i, j, t}}{L_{e f f, j}} \times N_{j}
\end{aligned}
$$

where $L_{e f f, j}$ is the average effective vehicle length at detector station $j, N$ is the total number of lanes at the detector station, and $m$ is the total number of detector records with free-flow traffic conditions within the same peak period. 
When the link is located at the head of a queue, it may be identified as partially queued (when the queue is identified upstream of the link but not downstream of the link). Therefore, the travel time estimation for a head of queue link consists of two parts: one for the queued section and one for the unqueued section, as shown below.

$$
T T_{i, t}=\frac{L_{i, 1} k_{i, 1, t}}{q_{i, 1, t}}+\frac{L_{i, 2}}{S_{i, 2, t}}
$$

where $L_{i, 1}$ is the length of queue section within the link, while $L_{i, 2}$ is the remaining uncongested section. Similar expression can be used for the travel time estimation of a tail of a queue link since such a link can be partially queued also.

While testing the above model, it was determined that the fast change in the queuing status during lane blockage incident conditions requires two refinements to the above model. The refined model is referred to as the Refined On-Line Model 1. The first refinement is to predict the queue length during the queue forming stage to account for the lag between the times the vehicles receive the information (e.g., at a Dynamic Message Sign location) and the times they arrive at the tail of the queue, as shown in Figure 3-8.

The predicted queue length is calculated based on the current location of the vehicle, current ending location of the queue and the propagation of the backward forming shock wave. This propagation speed is calculated as follows:

$$
\omega_{b}=\frac{q_{2}-q_{1}}{k_{2}-k_{1}}
$$


where $\omega_{b}$ denotes the shock wave speed, $q_{2}$ and $q_{1}$ are the flow rates within and upstream of the queue, respectively, and $k_{2}$ and $k_{1}$ are the corresponding densities. In this study, $q_{2}$ and $k_{2}$ are approximated by the flow and density at the downstream station of the tail of the queue, while $q_{1}$ and $k_{1}$ are the traffic parameters at the upstream station of this link. Note that when the downstream station of the tail of the queue is within the transition region, the parameters at the next downstream station will be used as $q_{2}$ and $k_{2}$. The speed within the queued section is estimated as the average speed of all of the detector stations within the queue. This speed is calculated as a function of flow and density.

time $t$

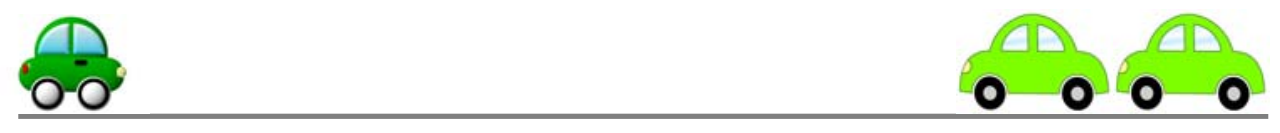

time $t+\Delta t$ :

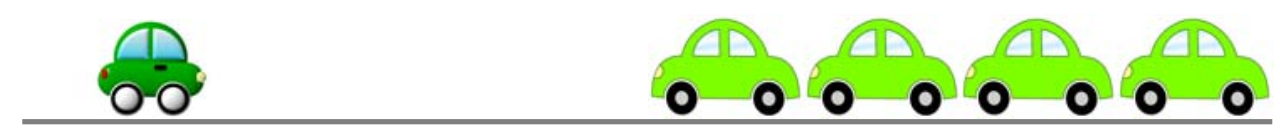

time $t+n \Delta t:$

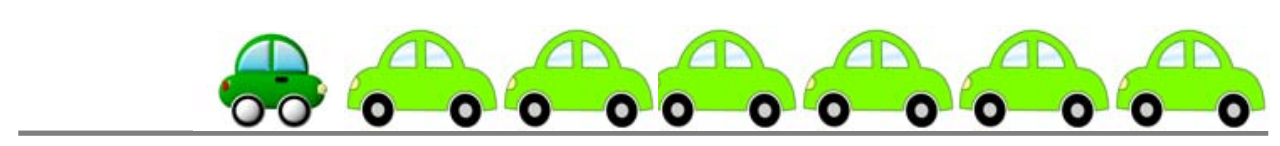

Figure 3-8 Illustration for the Need of Refinement 1

The second refinement to the model considers a front recovery shock wave a few minutes before the time at which the incident is forecast to be cleared. This refinement can be applied only when such forecasting is possible, for example, by an operator who is monitoring a real-time video display of the incident scene and communicating with the 
incident management team ${ }^{1}$. This refinement is to account for vehicles that receive travel time information at the DMS locations, with the travel time calculated under the assumption that the front of the queue due to the incident at a given location is fixed at that location, but in fact, because the traffic is in the recovery stage after the lane blockage is cleared, the queue length is decreasing with the head of the queue moving upstream due to the fast moving backward recovery shock wave. If this reduction in queue is not considered then the travel time received by the affected vehicles at the DMS location will be an overestimated travel time. Figure 3-9 presents an illustration of this situation.

time $t$ :

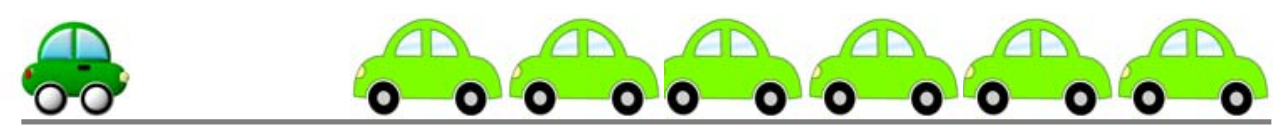

time $t+\Delta t$ :

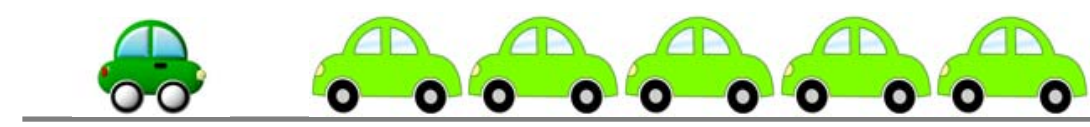

time $t+n \Delta t:$

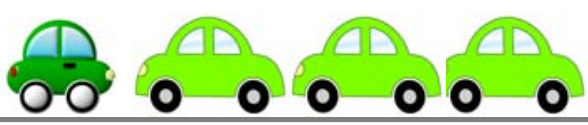

Figure 3-9 Illustration for the Need of Refinement 2

The refinement applied to address this issue includes the estimation of the recovery shock wave speed as follows:

$$
\omega_{f}=\frac{c_{2}-c_{1}}{k_{2}-k_{1}}
$$

${ }^{1}$ The utilization of an incident duration prediction model was also considered but eliminated from further consideration due to the expected variation in the durations of incidents with similar attributes. 
where $\omega_{f}$ represents the speed of the recovery shock wave, $c_{1}$ is the capacity during the incident, and $c_{2}$ is the queue discharge rate during incident clearance. These two parameters can be estimated from the normal roadway capacity and the capacity reduction factor during the incidents. The capacity and capacity reduction can be estimated based on the Highway Capacity Manual (HCM) procedures and parameters, or estimated based on detector station data, if this is possible. Parameters $k_{1}$ and $k_{2}$ are the corresponding densities. The reduction in queue length due to the recovery shock wave is then calculated based on the vehicle starting location, the time that incident starts to clear, and the speed of recovery shock wave. The average speed, determined from capacity $c_{2}$ and density $k_{2}$, is used for those recovered roadway segments.

It should be pointed out that at the final stage of this study, it was found that similar to this study, Yi (2009) developed a travel time estimation framework by combining a speed-based method with a traffic-flow theory based method and a statistics-based method. However, Yi's study is different from this study in that it requires 1-second detector data and the knowledge of the timestamps when each vehicle enters and exits the detection zone. This study requires 20-second detector data for speed, volume count and occupancy, which is the typical aggregation level of detector data in traffic management systems. Further, Yi (2009) only tested the algorithms for very short links with a length of up to 3,300 feet, which may not capture the impacts of queue propagation, and also did not test the algorithm during incident conditions. Finally, for on-line application, Yi's study did not take the dynamics of queue into consideration. 


\subsubsection{On-Line Hybrid Model 2}

Instead of combining a traffic flow method and a speed-based method as is done in On-Line Hybrid Model 1, Hybrid Model 2 combines two different speed-based methods: the Mid-Point method and the Minimum Speed method. The rational is that the literature review shows that the Mid-Point method underestimates travel time during congested conditions. Thus, using the minimum of the speeds measured at upstream and downstream detectors, as is done in the Minimum Speed method, may produce better results.

The existence of the queue and its status, identified by clustering analysis as explained above, can be used with the On-Line Hybrid Model 2 to select the appropriate method for travel time estimation, as follows:

- If there is no queue identified along a path, the traffic is under non-congested conditions and the Mid-Point method is applied to estimate the travel time for these conditions.

- When the queue exists and it is growing backward, the Minimum Speed method is selected for the travel time estimation to capture the dynamic growth of the queue. Since the Minimum Speed method uses the lower value of the upstream and downstream station speeds to represent the average link speed, it implicitly considers the queue propagation to the upstream station.

- If the queue is dissipating with a forward recovery shock wave, which occurs at the time when the recurrent congestion starts to dissipate at the bottleneck due to the reduction of upstream demands, the Minimum Speed method is also 
applied to account for the congestion considering that the recovery shock waves in this case is slow and not as fast as the recovery shock wave in case of incident clearance, which is described next.

- If the queue is dissipating with a backward recovery shock wave, such as in the case of incident clearance, the travel time estimation switches back to the Mid-Point method. This is because the fast moving recovery shock wave will result in a fast reduction in queue length and it is expected that there will be an overestimation if the Minimum Speed method is used in this case.

Similar to Hybrid Model 1, a refinement is applied to Hybrid Model 2 by including in the calculation a front recovery shock wave that is used to account for the incident recovery conditions. The procedures to estimate the impact of the recovery shock wave speed on the length of the congested region is the same as that used in Model 1. However, Model 2 does not need to account for the near-term growth of the queue length during the queue forming stage under incident conditions, as was done in the Refined Hybrid Model 1, since the Minimum Speed method already calculates the link travel time within the queue if the downstream detector station indicates the presence of a queue.

\subsection{Off-Line Travel Time Estimation}

Although this study mainly focuses on the real-time (on-line) estimation of travel time, for comparison purposes and for potential use for off-line applications, corresponding hybrid off-line estimation models that utilize historical data are also developed. This is also useful since off-line estimates have been used in the training process of real-time 
short-term travel time prediction methods such as Neural Network, Time Series, Regression, and Nearest Neighbor.

The difference between on-line and off-line estimation methods is that for on-line applications, future traffic conditions along the paths of the vehicle are not available and only the instantaneous travel time (based on the traffic conditions at the time of the estimation) can be used in the estimation. For off-line estimation, the traffic conditions at later time periods are determined based on historical data. Thus, the actual travel time experienced by the vehicles can be estimated based on traffic conditions, as the vehicle progresses in its route from one link to the next.

The method used in this study divides the whole time duration into small time periods that are critical to the temporal aggregation level of the detector data, as shown in Figure 3-10.

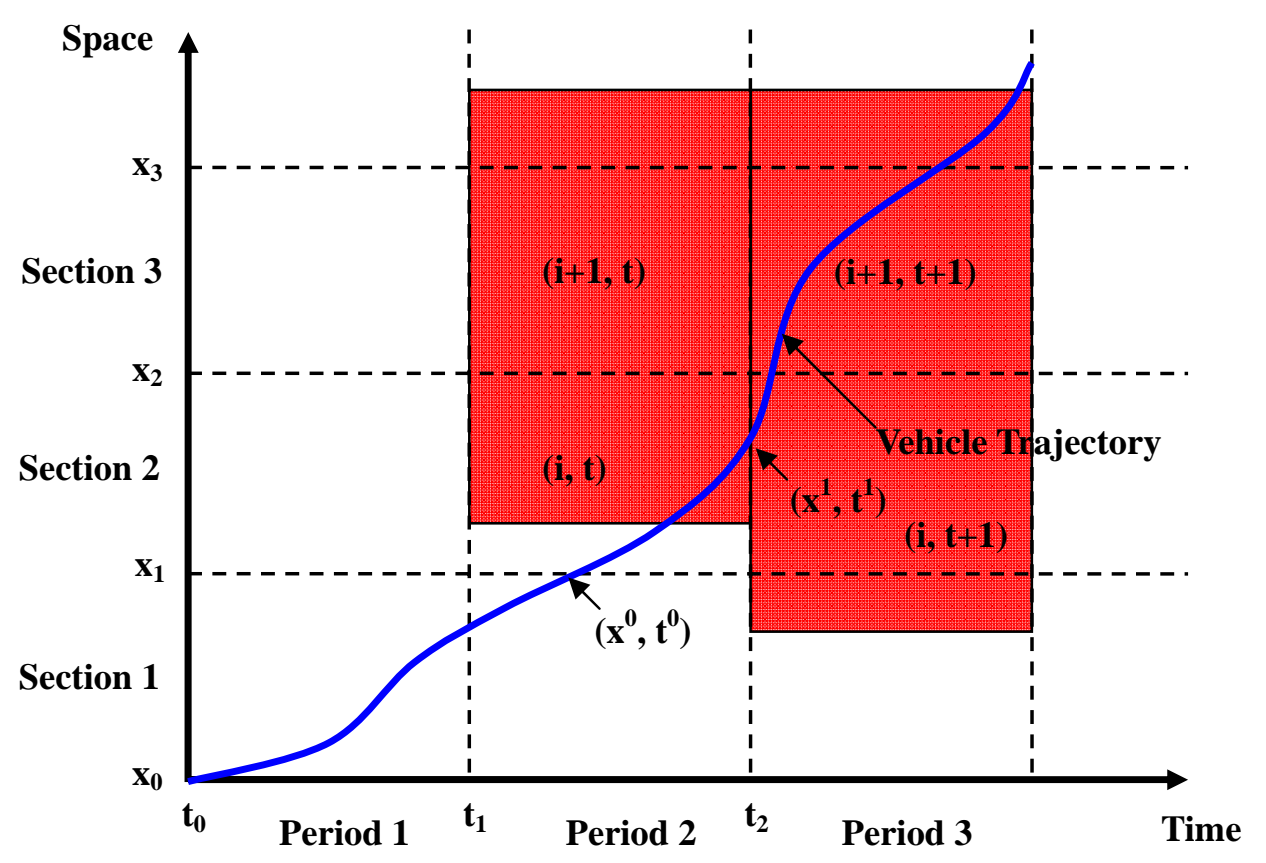

Figure 3-10 Schematic Diagram for Off-Line Travel Time Estimation 
As shown in this figure, a vehicle enters cell $i$ at location $x^{0}$ and time $t^{0}$. The remaining time in this time period is compared to the time that is required to reach the downstream station, and the minimum value of these two values is used in the travel time estimation for this cell. Depending on the location of the exit point $\left(x^{1}, t^{1}\right)$, the vehicle can either enter the next link during the same period, which is cell $(i+1, t)$; stay on the same link but experience different traffic conditions at time $t+1$, which is cell $(i, t+1)$; or enter the downstream link at the next period of time, which is cell $(i+1, t+1)$. The resulting route travel time of a vehicle is the time that the vehicle arrives at the destination (last detection station on the path for which the travel time is estimated) minus the time that the vehicle departs from the origin (first detection station on the path for which the travel time is estimated). This concept is similar to the concept presented by Van Lint (2004). However, instead of using the Piece-wise Linear Speed method as was done in the study by Van Lint (2004), the hybrid methods developed in this study are used to calculate the travel time within each cell. In parallel to the two on-line hybrid models mentioned above, two hybrid off-line models are also developed, which are explained below.

\subsubsection{Off-Line Hybrid Model 1}

Similar to the On-Line Hybrid Model 1, the travel time within each cell for the Off-Line Hybrid Model 1 are either estimated by the Mid-Point method or Flow-based method, depending on the congestion level identified by the clustering analysis, as described above. Given the entry location and time $\left(x^{0}, t^{0}\right)$ at each cell, the exit location and time $\left(x^{1}, t^{1}\right)$ can be written as follows: 


$$
\left(x^{1}, t^{1}\right)= \begin{cases}\left(x_{d}, t\left(x_{d}\right)\right) & t\left(x_{d}\right) \leq t_{p} \\ \left(x\left(t_{p}\right), t_{p}\right) & t\left(x_{d}\right)>t_{p}\end{cases}
$$

where $x_{d}$ indicates the downstream detector location for each cell, $t_{p}$ refers to the ending timestamp for the cell, which corresponds to the time of the next travel time update, $t\left(x_{d}\right)$ is the timestamp when reaching the downstream detector location, and $x\left(t_{p}\right)$ is the location that can be reached by the vehicles at timestamp $t_{p}$. The expressions for these two parameters vary with the congestion level in the cell. Below is a detailed discussion of how to calculate $t\left(x_{d}\right)$ and $x\left(t_{p}\right)$ under different conditions.

\section{Case 1: The cell is free of congestion.}

In this case, the Mid-Point method is used for travel time estimation as it performs well under uncongested conditions. Since the Mid-Point method assumes that each detector speed measurement represents the speeds of half the distance to the next detector on both sides, the travel time estimation in this case is divided into two parts, depending on whether the entry point of vehicles $x^{0}$ is within the first half of the cell or the second half of the cell.

If the entry point of vehicles $x^{0}$ is within the second half of the cell, that is,

$$
x^{0} \geq x_{u}+\frac{1}{2}\left(x_{d}-x_{u}\right)
$$

where $x_{u}$ indicates the upstream detector locations; then the corresponding timestamp when reaching the downstream detector location $t\left(x_{d}\right)$ is expressed as:

$$
t\left(x_{d}\right)=t^{0}+\frac{x_{d}-x^{0}}{S_{d}}
$$

The location $x\left(t_{p}\right)$ that can be reached by the vehicles at the timestamp $t_{p}$ is 


$$
x\left(t_{p}\right)=x_{0}+\left(t_{p}-t^{0}\right) \times S_{d}
$$

In the case that the entry point of vehicles is within the first half of the cell, as expressed below,

$$
x^{0}<x_{u}+\frac{1}{2}\left(x_{d}-x_{u}\right)
$$

The expression for $t\left(x_{d}\right)$ is

$$
t\left(x_{d}\right)=t^{0}+\frac{x_{u}+\frac{1}{2}\left(x_{d}-x_{u}\right)-x^{0}}{S_{u}}+\frac{\frac{1}{2}\left(x_{d}-x_{u}\right)}{S_{d}}
$$

The location $x\left(t_{p}\right)$ at timestamp $t_{p}$ is then written as:

$$
x\left(t_{p}\right)= \begin{cases}x^{0}+S_{u} \times\left(t\left(x_{m}\right)-t^{0}\right)+S_{d} \times\left(t_{p}-t\left(x_{m}\right)\right) & t\left(x_{m}\right) \leq t_{p} \\ x^{0}+S_{u} \times\left(t_{p}-t^{0}\right) & t\left(x_{m}\right)>t_{p}\end{cases}
$$

where $t\left(x_{m}\right)$ is the timestamp when the vehicles reach the mid-point of the cell, which is calculated by the following:

$$
t\left(x_{m}\right)=t^{0}+\frac{x_{u}+\frac{1}{2}\left(x_{d}-x_{u}\right)-x^{0}}{S_{u}}
$$

\section{Case 2: The cell is in-queue.}

When the status of the cell is in-queue, the flow-based method is applied to calculate $t\left(x_{d}\right)$ and $x\left(t_{p}\right)$ as follows:

$$
\begin{aligned}
& t\left(x_{d}\right)=t^{0}+\left(x_{d}-x^{0}\right) \times \frac{\left(k_{u}+k_{d}\right)}{2 q_{d}} \\
& x_{d}\left(t_{p}\right)=x^{0}+\frac{2 q_{d}}{\left(k_{u}+k_{d}\right)} \times\left(t_{p}-t^{0}\right)
\end{aligned}
$$


where $k_{u}$ and $k_{d}$ are the densities at the upstream and downstream detector locations, respectively. $q_{d}$ represents the flow rate at the downstream detector location of this cell.

Case 3: The cell is the head of a queue.

Similar to the On-Line Hybrid Model 1, the head of queue cell consists of two parts, one within the queue and one outside the queue, that is, an uncongested part downstream of the congestion. If the vehicles enter the cell in the uncongested part, the values of $t\left(x_{d}\right)$ and $x\left(t_{p}\right)$ are obtained from the downstream detector speed, that is,

$$
\begin{aligned}
& t\left(x_{d}\right)=t^{0}+\frac{x_{d}-x^{0}}{S_{d}} \\
& x\left(t_{p}\right)=x_{0}+\left(t_{p}-t^{0}\right) \times S_{d}
\end{aligned}
$$

However, if the vehicles enter the cell within the congested part, the corresponding expressions for $t\left(x_{d}\right)$ and $x\left(t_{p}\right)$ are,

$$
\begin{gathered}
t\left(x_{d}\right)=t^{0}+\left(x_{q}-x^{0}\right) \times \frac{k_{u}}{q_{u}}+\frac{x_{d}-x_{q}}{S_{d}} \\
x\left(t_{p}\right)= \begin{cases}x^{0}+\frac{q_{u}}{k_{u}} \times\left(t\left(x_{q}\right)-t^{0}\right)+S_{d} \times\left(t_{p}-t\left(x_{q}\right)\right) & t\left(x_{q}\right) \leq t_{p} \\
x^{0}+\frac{q_{u}}{k_{u}} \times\left(t_{p}-t^{0}\right) & t\left(x_{q}\right)>t_{p}\end{cases}
\end{gathered}
$$

where $x_{q}$ is the ending location of the queue within the cell and $t\left(x_{q}\right)$ is the timestamp when the vehicles reach the location $x_{q}$. The vehicles may exit the cell from either the congested or uncongested region, and thus two different expressions are formulated for $x\left(t_{p}\right)$ in Equation 3-32. 


\section{Case 4: The cell is the tail of a queue.}

Contrary to the head of a queue case described above, the first part of a tail of a queue cell is uncongested while the second part of this cell is within the queue. The method used for the estimation of travel time for a tail of queue cell follows the same idea as the head of queue cell.

If the entering location $x^{0}$ of a vehicle is within a congested region, the expressions for $t\left(x_{d}\right)$ and $x\left(t_{p}\right)$ are written as

$$
\begin{aligned}
& t\left(x_{d}\right)=t^{0}+\left(x_{d}-x^{0}\right) \times \frac{k_{d}}{q_{d}} \\
& x_{d}\left(t_{p}\right)=x^{0}+\frac{q_{d}}{k_{d}} \times\left(t_{p}-t^{0}\right)
\end{aligned}
$$

Otherwise, the corresponding expressions are

$$
\begin{gathered}
t\left(x_{d}\right)=t^{0}+\frac{x_{q}-x^{0}}{S_{u}}+\left(x_{d}-x_{q}\right) \times \frac{k_{d}}{q_{d}} \\
x\left(t_{p}\right)= \begin{cases}x^{0}+S_{u} \times\left(t\left(x_{q}\right)-t^{0}\right)+\frac{q_{d}}{k_{d}} \times\left(t_{p}-t\left(x_{q}\right)\right) & t\left(x_{q}\right) \leq t_{p} \\
x^{0}+S_{u} \times\left(t_{p}-t^{0}\right) & t\left(x_{q}\right)>t_{p}\end{cases}
\end{gathered}
$$

where $x_{q}$ is the starting location of the queue within the cell and $t\left(x_{q}\right)$ is the timestamp when the vehicles reach the location of $x_{q}$.

As mentioned above, the resulting route travel time of a vehicle is the time that the vehicle arrives at the destination (last detection station on the path for which the travel time is estimated) minus the time that the vehicle departs the origin (first detection station on the path for which the travel time is estimated). 


\subsubsection{Off-Line Hybrid Model 2}

The Off-Line Hybrid Model 2 utilizes the Mid-Point method for uncongested cells and the Minimum Speed method for the fully or partially congested cells. The combination of these two speed-based methods aims at utilizing the advantages of each individual estimation methods and thus improving the overall estimation performance.

With the Off-Line Hybrid Model 2, the exit location and exit time $\left(x^{1}, t^{1}\right)$ can be expressed as the function of entry location and time $\left(x^{0}, t^{0}\right)$ as in Equation 3-19. For the uncongested cells, the expressions for $t\left(x_{d}\right)$ and $x\left(t_{p}\right)$ in this equation are exactly the same as those described in Model 1 and are omitted here for brevity.

The expressions of $t\left(x_{d}\right)$ and $x\left(t_{p}\right)$ for fully congested or partially congested cells are as follows:

$$
\begin{aligned}
& t\left(x_{d}\right)=t^{0}+\frac{x_{d}-x^{0}}{\min \left(S_{u}, S_{d}\right)} \\
& x\left(t_{p}\right)=x_{0}+\left(t_{p}-t^{0}\right) \times \min \left(S_{u}, S_{d}\right)
\end{aligned}
$$

Note that since the traffic parameters at later time periods are known, the off-line hybrid models do not need the refinements to account for fast moving shock waves as the on-line hybrid models. Compared to the previous travel time estimation studies (Dhulipala 2002; Xia and Chen 2007), the hybrid off-line models developed in this study do not need additional information about incidents, such as the incident occurrence time and duration, since the clustering analysis described above will automatically detect the occurrence and disappearance of queue. 


\subsection{Summary}

In this chapter, two on-line as well as two off-line hybrid freeway travel time estimation models were developed based on point detector measurements. Before exercising the model, detector data is checked, smoothed, aggregated, and imputed. One of the important components of the developed models is incorporating clustering analysis in the travel time estimation model to identify traffic conditions. This is a new contribution from this study. Based on congestion level and queue status identified by the clustering analysis, different travel time estimation methods are selected in the developed models to take advantage of each individual estimation method. Hybrid Model 1 uses the Mid-Point speed-based method for estimating travel time along uncongested links, and a traffic flow-based method for travel time on congested links. Hybrid Model 2 combines two speed-based methods: the Mid-Point method for the uncongested condition and the Minimum Speed method for congested conditions. Variations of these models are applied to real-time and off-line conditions, with the off-line versions taking advantage of the travel time at future time steps that are known for off-line applications, but not real-time applications. For real-time applications, refinements to the hybrid models are developed based on shock wave analysis to capture the dynamics of queue propagation. 


\section{CHAPTER 4}

\section{MODEL ASSESSMENT AND COMPARISON}

The accuracy and reliability of travel time estimates obtained using various existing speed-based methods including the SunGuide algorithm, a simple traffic flow-based method based on flow and occupancy, the modified N-D method (a traffic flow method), and the hybrid models described in Chapter 3, are evaluated and compared in this study. The comparisons are made using simulation models as well as real-world travel time data. The description of existing methods included in the comparison of this study can be found in the literature review section in Chapter 2.

\subsection{Study Corridor}

Figure 4-1 shows the corridor used as a case study in the comparison. The study corridor is the eastbound section of SR-826, located in Miami-Dade County, Florida, starting from the location of detector DS-1509E to the location of detector DS-1549E. It includes six interchanges with a total length of about 6.4 miles. As shown in Figure 4-1, there are 21 true presence microwave detector stations deployed along this section with a spacing that ranges from between 0.3 to 0.5 miles. However, it is found that detector station DS-1513E reported erroneous data during the period of the study, and therefore this station is excluded from the analysis. 


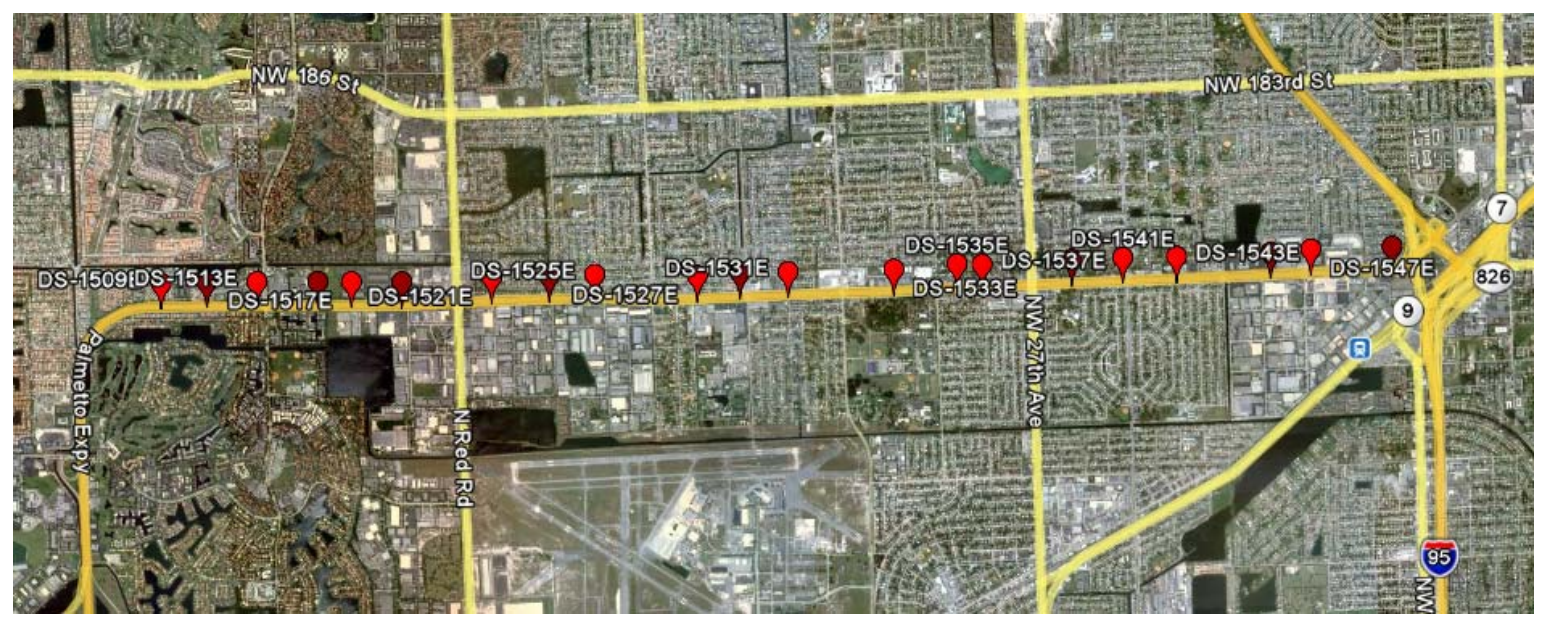

Figure 4-1 Study Corridor and Detector Locations

\subsection{Performance Measures}

Two performance measures are used to quantify the accuracy of the estimated travel times. These performance measures are the mean absolute error (MAE) and mean absolute percentage error (MAPE). These two performance measures are defined as follows:

$$
\begin{aligned}
& M A E=\frac{1}{N} \sum_{i=1}^{N}\left|T T_{i}-T T_{i, a}\right| \\
& M A P E=100 \frac{1}{N} \sum_{i=1}^{N} \frac{\left|T T_{i}-T T_{i, a}\right|}{T T_{i, a}}
\end{aligned}
$$

where $T T_{i}$ is the $i^{\text {th }}$ estimated travel time, and $T T_{i, a}$ is the corresponding real-world or simulated travel time (depending on the source of the ground truth data). $N$ is the total number of estimates.

Because FDOT districts generally post ranges of travel time values rather than fixed travel time values on their traveler information devices, it is necessary to quantify the reliability of the estimated travel times, as well. In this study, the reliability of travel 
time estimates is defined as the percentage of vehicles with travel times that are within the range of travel time posted on the traveler information devices. In addition, the percentage of vehicles with travel times that are less than the posted minimum travel time (referred to as percentage early in this study) as well as the percentage of vehicles with travel time greater than the posted maximum travel time (referred to as percentage late) is also reported to differentiate between these two conditions. This study calculates the travel time ranges that are displayed to travelers using the same method used by the traffic management centers (TMCs) in South Florida in their real-world operations (Florida Department of Transportation District 6, 2010). With this calculation, if the estimated travel time is less than five minutes, the traveler information message to travelers is "Under 5 Minutes". If the travel time is more than 35 minutes, the message is "Over 35 Minutes". A 3-minute range is used when the estimated travel time is between 5 minutes and 10 minutes, and a 5-minute range is used for travel times between 10 minutes and 35 minutes.

\subsection{Assessment Based on Simulation Data}

First, the different travel time estimation methods were tested using a simulation model that is calibrated for incident and no-incident conditions. The utilized simulation tool is the CORSIM microscopic simulation tool. The development and calibration of simulation models using data collected from ITS have been addressed as part of a separate FDOT research project conducted by Hadi et al. (2010). The details of these procedures can be found in the final report of the research project mentioned above and will not be repeated here. 
Three scenarios were used in the comparison. The first scenario represents uncongested conditions and the other two represent non-recurrent congestions caused by one-lane blockage incidents with different attributes. It should be noted that the comparison based on simulation presented in this section assumes that all detector measurements have $100 \%$ accuracy. This assumption is relaxed in the next chapter to determine the impacts of detector errors on the results. The temporal aggregation level of travel time used in the comparison is two minutes, which is a common aggregation level used in travel time estimation. The SunGuide system can be configured to estimate travel time based on the Mid-Point and Point-to-Point methods. The measured speeds are capped by the speed limit (for SR-826, the speed limit is $55 \mathrm{mph}$ ) in the SunGuide system before being used in the travel time calculation. The comparison with the SunGuide estimation is done with and without the capping of the speed based on the Point-to-Point method.

\subsubsection{Simulated Uncongested Scenario}

Figure 4-2 presents the travel time results for uncongested conditions and Table 4-1 shows the accuracy and reliability of various on-line travel time estimation methods for this scenario. The improved N-D method in Table 4-1 refers to the method developed by Vanajakshi (2009), which was selected as an example of the latest traffic flow theory-based methods that can be found in the literature. The flow-based method in this table is a method that uses the volume count and occupancy to estimate the travel time as described in Equation 3-13. The purpose of including this method is to examine the 
performance of the flow-based estimation method when not combined with speed-based methods.

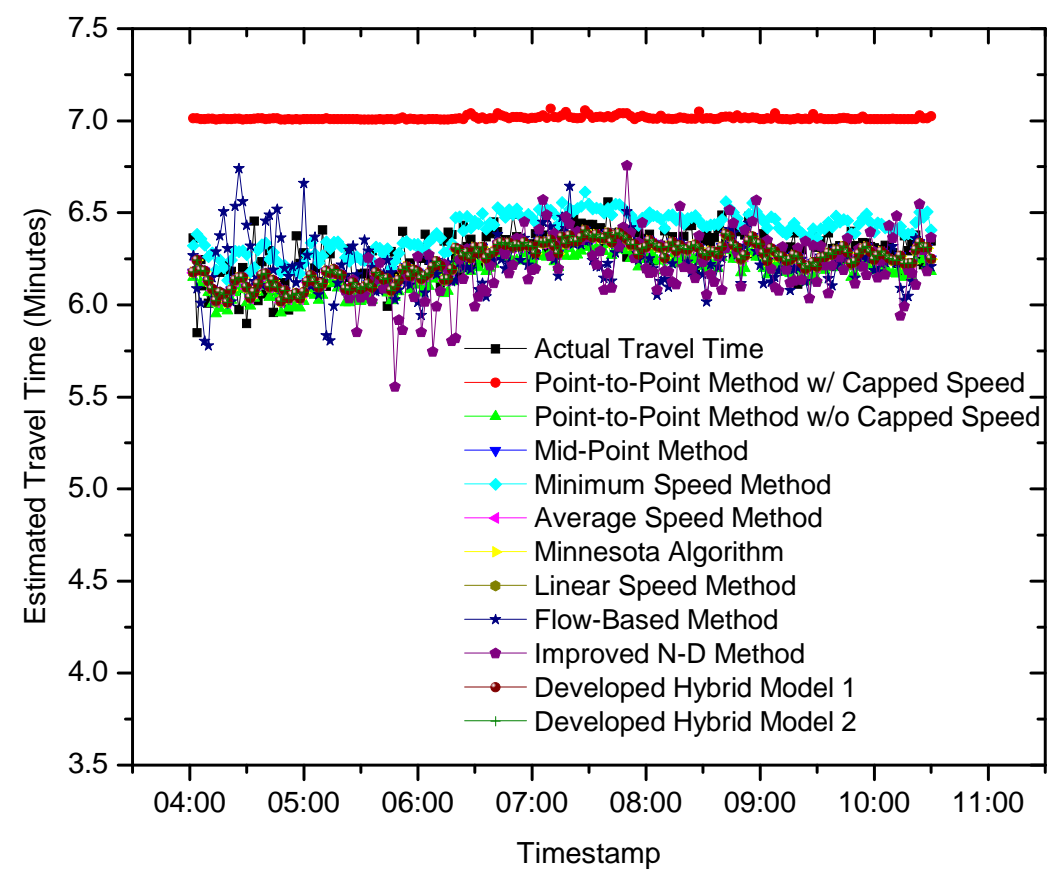

(a) On-Line Estimation Results

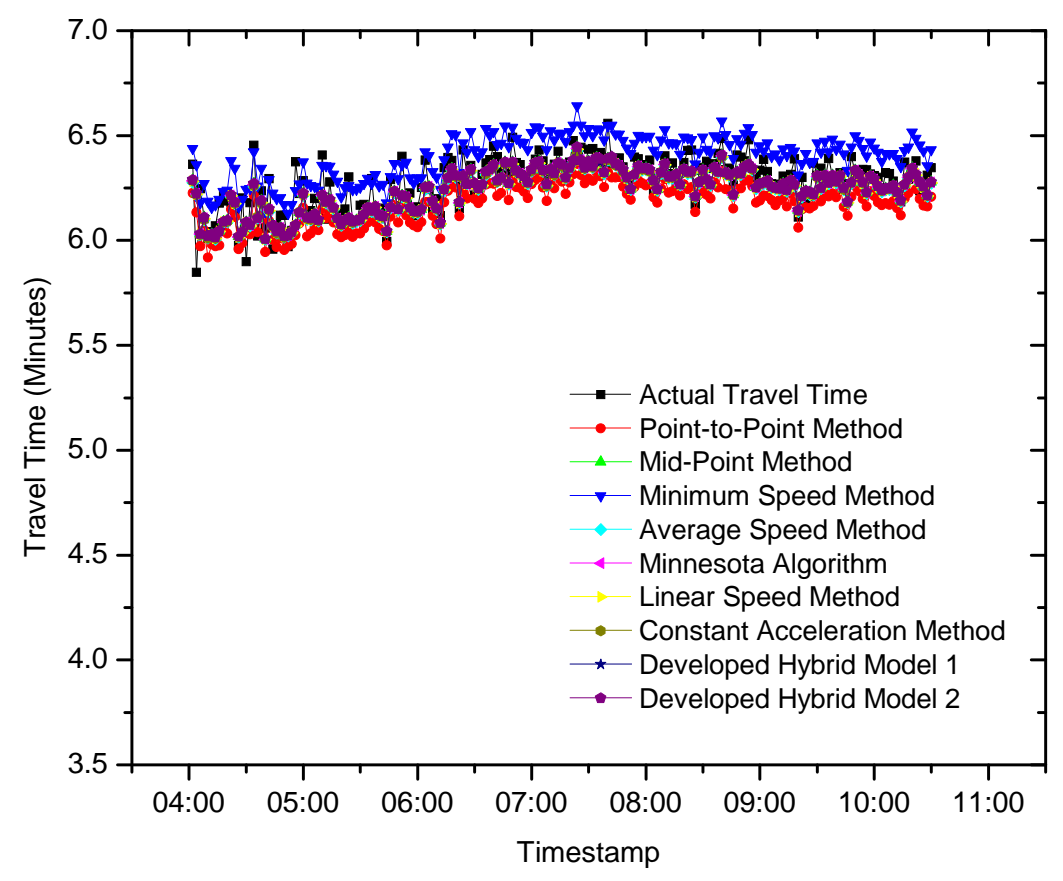

(b) Off-Line Estimation Results

Figure 4-2 Estimated Travel Time for Simulated Uncongested Condition 
As show in Table 4-1, almost all of the on-line travel time methods can achieve good accuracy and reliability during uncongested conditions except the Point-to-Point method with capped speed, which overestimates the travel time due to the capped speed. The comparison among the different speed-based methods shows that the Minimum Speed method has slightly higher errors and lower reliability relative to the other speed-based methods, although it still performs well. It also can be seen in Table 4-1 that the developed models perform well in this case. Compared to the on-line estimation methods, the off-line methods can achieve slightly better estimation performance, as shown in Table 4-2. However, this improvement is not significant.

Table 4-1 Accuracy and Reliability of Tested On-Line Travel Time Estimation Methods for Simulated Uncongested Condition

\begin{tabular}{|l|c|c|c|c|c|}
\hline Method & $\begin{array}{c}\text { MAE } \\
\text { (Min.) }\end{array}$ & $\begin{array}{c}\text { MAPE } \\
\mathbf{( \% )}\end{array}$ & $\begin{array}{c}\text { Reliability } \\
\mathbf{( \% )}\end{array}$ & $\begin{array}{c}\text { \% } \\
\text { Early }\end{array}$ & \% Late \\
\hline Point-to-Point Method w/ Capped Speed & 0.74 & 11.77 & 80.59 & 19.41 & 0 \\
\hline Point-to-Point Method w/o Capped Speed & 0.12 & 1.92 & 100 & 0 & 0 \\
\hline Mid-Point Method & 0.08 & 1.31 & 100 & 0 & 0 \\
\hline Minimum Speed Method & 0.14 & 2.18 & 97.58 & 2.42 & 0 \\
\hline Average Speed Method & 0.08 & 1.34 & 100 & 0 & 0 \\
\hline Minnesota Method & 0.08 & 1.32 & 100 & 0 & 0 \\
\hline Linear Speed Method & 0.08 & 1.33 & 100 & 0 & 0 \\
\hline Flow-Based Method & 0.15 & 2.38 & 99.5 & 0.44 & 0 \\
\hline Improved N-D Method & 0.15 & 2.38 & 99.29 & 0.71 & 0 \\
\hline Developed Hybrid Model 1 & 0.08 & 1.31 & 100 & 0 & 0 \\
\hline Developed Hybrid Model 2 & 0.08 & 1.31 & 100 & 0 & 0 \\
\hline
\end{tabular}


Table 4-2 Accuracy and Reliability of Tested Off-Line Travel Time Estimation Methods for Simulated Uncongested Condition

\begin{tabular}{|l|c|c|c|c|c|}
\hline Method & $\begin{array}{c}\text { MAE } \\
\text { (Min.) }\end{array}$ & $\begin{array}{c}\text { MAPE } \\
(\mathbf{\%})\end{array}$ & $\begin{array}{c}\text { Reliability } \\
(\mathbf{\%})\end{array}$ & \% Early & \% Late \\
\hline Point-to-Point Method & 0.11 & 1.76 & 100 & 0 & 0 \\
\hline Mid-Point Method & 0.06 & 0.94 & 100 & 0 & 0 \\
\hline Minimum Speed Method & 0.12 & 1.99 & 97.83 & 2.17 & 0 \\
\hline Average Speed Method & 0.06 & 0.99 & 100 & 0 & 0 \\
\hline Minnesota Method & 0.06 & 0.95 & 100 & 0 & 0 \\
\hline Linear Speed Method & 0.06 & 0.97 & 100 & 0 & 0 \\
\hline Constant Acceleration Method & 0.06 & 0.96 & 100 & 0 & 0 \\
\hline Developed Hybrid Model 1 & 0.06 & 0.94 & 100 & 0 & 0 \\
\hline Developed Hybrid Model 2 & 0.06 & 0.94 & 100 & 0 & 0 \\
\hline
\end{tabular}

\subsubsection{Simulated Incident Scenario 1}

Figure 4-3(a) presents the results of the on-line travel time estimation for one of the incident scenarios used as a case study and referred to as simulated incident scenario 1 in the discussion. In this simulation case, a one-lane blockage incident occurs at 7:35 A.M. and lasts for 25 minutes. For clarity, Figure 4-3(b) presents the same results presented in Figure 4-3(a) but only for the Point-to-Point method with capped speeds (i.e., SunGuide method), the on-line Minimum Speed method, and the on-line hybrid models developed in this study. Figure 4-3(c) shows the corresponding off-line travel time estimation results. Figure 4-3(a) shows that, unlike the uncongested case, the results obtained from different travel time estimation methods vary significantly. It appears that the Minimum Speed method is comparable to the other speed-based methods for this incident scenario. The flow-based method and the improved N-D method can also produce relatively good results. However, these three methods (Minimum Speed, Flow-based method and the improved N-D method) overestimate the travel time at the later stage of lane blockage due to the effect of the front recovery shock wave, described 
earlier. Figure 4-3(a) also includes the results obtained from the hybrid models developed in this study without refinements, which are slightly to moderately better than those of the flow-based method and the Minimum Speed method but also suffers from the front recovery shock wave effect. The developed refined on-line models that consider the front recovery shock wave performed better than the other methods, as shown in Figure 4-3(b). Figure 4-3(b) also shows that the SunGuide method does not perform well and significantly underestimates the travel time under this incident scenario. Please note that in all the figures presented in this chapter, the developed Hybrid Models 1 and 2 referred to the models with the refinement described in the previous chapter.

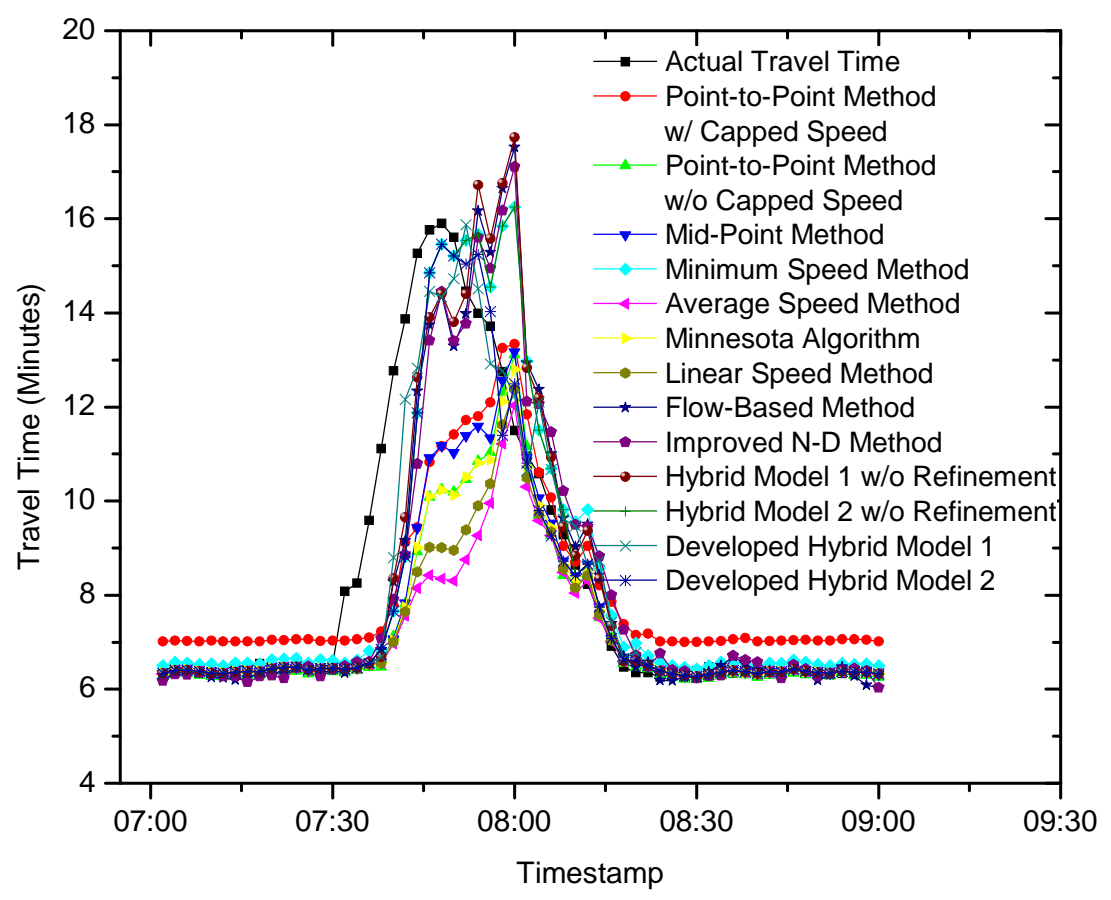

(a) On-Line Estimation Results

Figure 4-3 Estimated Travel Time for Simulated Incident Scenario 1

(continued on next page) 


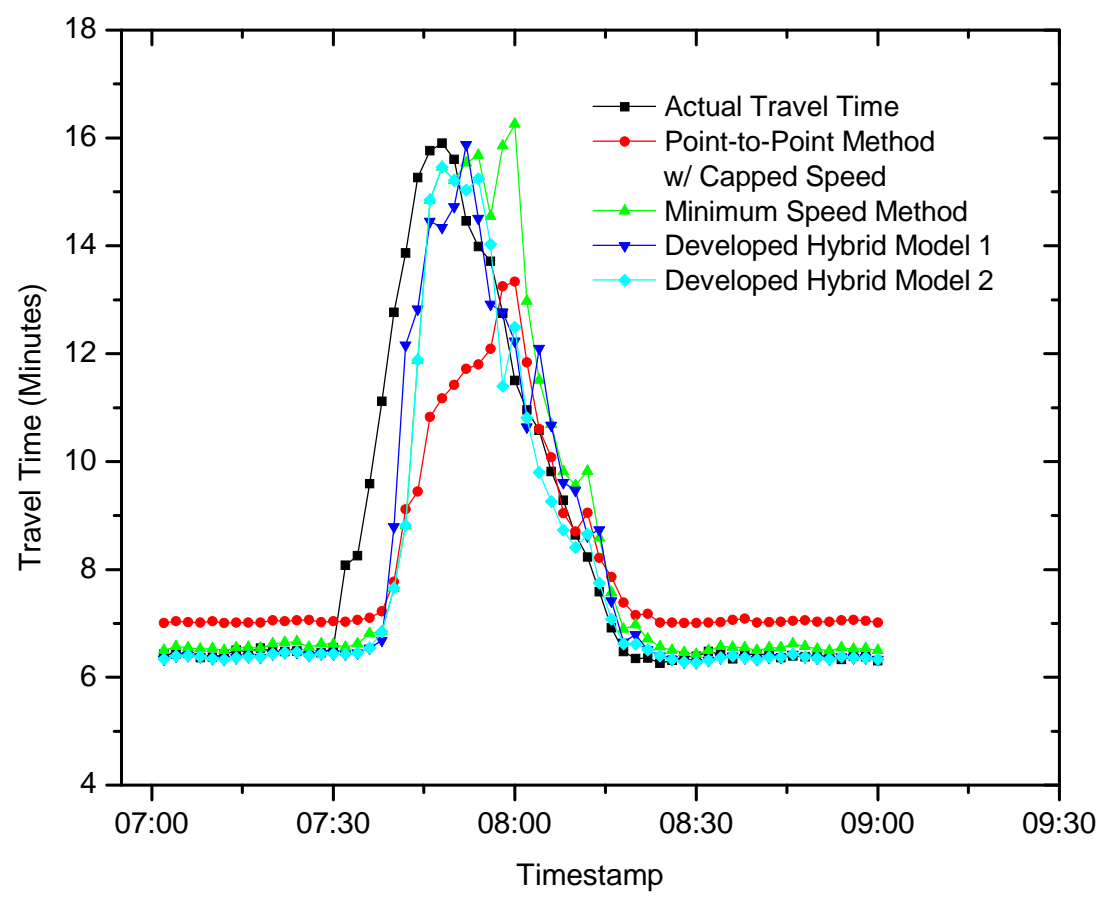

(b) On-Line Estimation Results

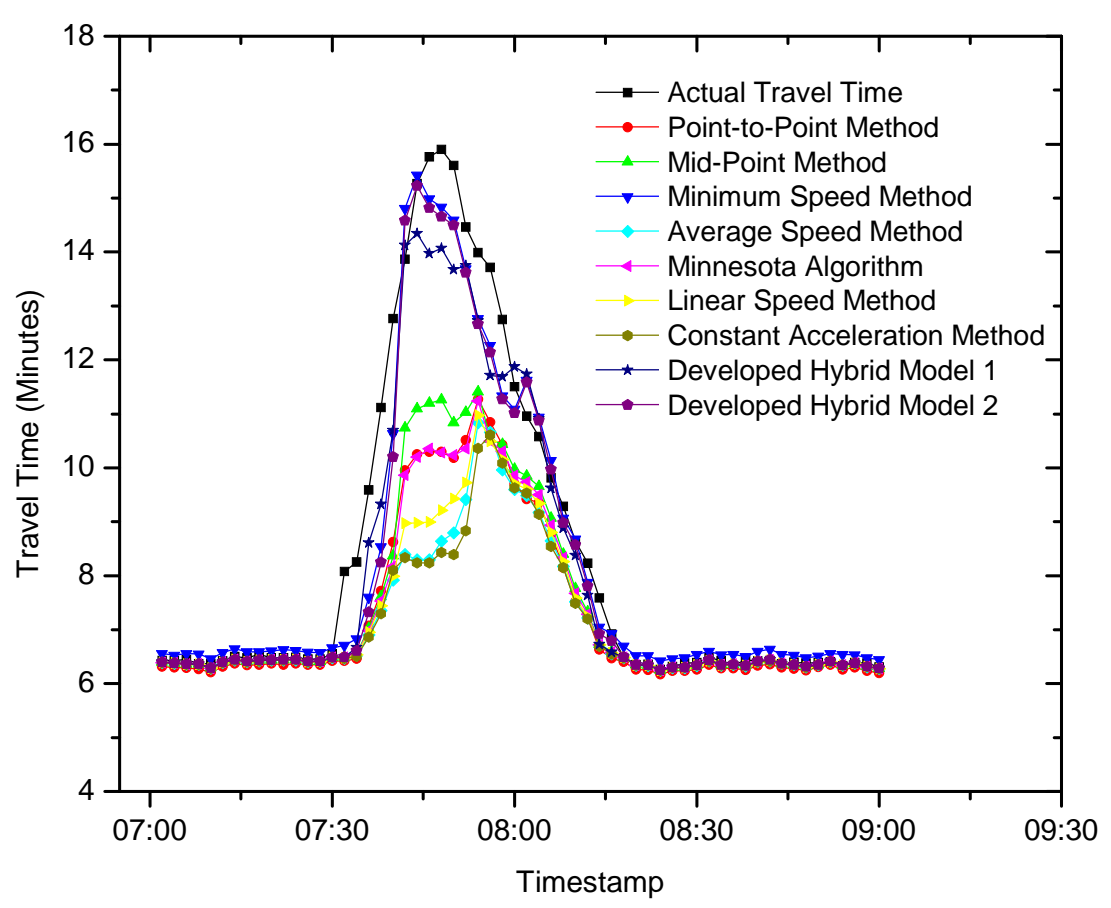

(c) Off-Line Estimation Results

Figure 4-3 Estimated Travel Time for Simulated Incident Scenario 1 
Table 4-3a lists the accuracy and reliability of each on-line travel time estimation method for this simulated incident case between 7:00 A.M. and 9:00 A.M. As stated previously, the lane blockage incident occurs at 7:35 A.M. and lasts for 25 minutes. It can be seen from this table that in general the accuracy and reliability of the estimated travel time during the incident conditions are not as good as those for uncongested conditions. The MAPE of travel time estimated by the SunGuide method with capped speed is about $12.79 \%$ and the corresponding reliability is $76.54 \%$ with $7.72 \%$ vehicles arriving early and $15.74 \%$ vehicles arriving late. Table 4 -3a also shows that the performances of the compared speed-based methods and flow-based methods are close. Compared to the other methods, the two developed hybrid models have slightly less errors and higher reliability even without the refinements. With the refinement, the accuracy and reliability of the hybrid models improved even further. A comparison of the results of the developed Hybrid Model 1 and Hybrid Model 2 shows that Model 2 performs slightly better than Model 1 .

Table 4-3b presents the performances of the on-line travel time estimation methods only between 7:30 A.M. and 8:30 A.M., as the time period from 7:00 A.M. to 9:00 A.M. includes partly uncongested conditions, while the traffic during the period between 7:30 A.M. and 8:30 A.M. is completely congested. Compared to the results for the time period 7:00 A.M. to 9:00 A.M., the errors during the time period 7:30 A.M. to 8:30 A.M. are higher and the reliabilities are lower. Table 4-3b indicates that the selection of the study period for comparison has a great impact on the evaluation of travel time estimation performance. 
Table 4-3a Accuracy and Reliability of Tested On-Line Travel Time Estimation Methods for Simulated Incident Scenario 1 between 7:00 A.M. and 9:00 A.M.

\begin{tabular}{|l|c|c|c|c|c|}
\hline Method & $\begin{array}{c}\text { MAE } \\
(\text { Min.) }\end{array}$ & $\begin{array}{c}\text { MAPE } \\
(\mathbf{\%})\end{array}$ & $\begin{array}{c}\text { Reliability } \\
(\%)\end{array}$ & $\begin{array}{c}\text { \% } \\
\text { Early }\end{array}$ & \% Late \\
\hline Point-to-Point Method w/ Capped Speed & 1.24 & 12.79 & 76.54 & 7.72 & 15.74 \\
\hline Point-to-Point Method w/o Capped Speed & 1.10 & 9.07 & 79.66 & 0.85 & 19.49 \\
\hline Mid-Point Method & 0.96 & 7.83 & 80.48 & 2.00 & 17.52 \\
\hline Minimum Speed Method & 0.86 & 8.23 & 75.53 & 15.55 & 8.92 \\
\hline Average Speed Method & 1.26 & 9.88 & 78.24 & 0.58 & 21.19 \\
\hline Minnesota Method & 1.06 & 8.50 & 80.18 & 1.64 & 18.18 \\
\hline Linear Speed Method & 1.18 & 9.32 & 78.70 & 0.58 & 20.72 \\
\hline Flow-Based Method & 0.94 & 8.64 & 80.21 & 8.76 & 11.03 \\
\hline Improved N-D Method & 0.99 & 9.30 & 79.93 & 9.20 & 10.87 \\
\hline Hybrid Model 1 w/o Refinement & 0.87 & 7.76 & 82.26 & 7.45 & 10.29 \\
\hline Hybrid Model 2 w/o Refinement & 0.76 & 6.80 & 82.23 & 7.88 & 9.88 \\
\hline Developed Hybrid Model 1 & 0.58 & 5.55 & 85.98 & 4.93 & 9.09 \\
\hline Developed Hybrid Model 2 & 0.60 & 5.50 & 87.85 & 1.59 & 10.57 \\
\hline
\end{tabular}

Table 4-3b Accuracy and Reliability of Tested On-Line Travel Time Estimation Methods for Simulated Incident Scenario 1 between 7:30 A.M. and 8:30 A.M.

\begin{tabular}{|l|c|c|c|c|c|}
\hline Method & $\begin{array}{c}\text { MAE } \\
\text { (Min.) }\end{array}$ & $\begin{array}{c}\text { MAPE } \\
(\mathbf{\%})\end{array}$ & $\begin{array}{c}\text { Reliability } \\
(\%)\end{array}$ & $\begin{array}{c}\text { \% } \\
\text { Early }\end{array}$ & \% Late \\
\hline Point-to-Point Method w/ Capped Speed & 1.87 & 16.08 & 57.90 & 9.08 & 33.03 \\
\hline Point-to-Point Method w/o Capped Speed & 2.07 & 16.28 & 57.32 & 1.78 & 40.90 \\
\hline Mid-Point Method & 1.86 & 14.78 & 59.05 & 4.19 & 36.76 \\
\hline Minimum Speed Method & 1.58 & 14.43 & 55.20 & 26.08 & 18.73 \\
\hline Average Speed Method & 2.45 & 18.81 & 54.34 & 1.21 & 44.46 \\
\hline Minnesota Method & 2.05 & 16.10 & 58.42 & 3.45 & 38.14 \\
\hline Linear Speed Method & 2.29 & 17.72 & 55.31 & 1.21 & 43.48 \\
\hline Flow-Based Method & 1.74 & 15.21 & 59.33 & 17.52 & 23.15 \\
\hline Improved N-D Method & 1.83 & 16.34 & 58.82 & 18.38 & 22.80 \\
\hline Hybrid Model 1 w/o Refinement & 1.68 & 14.65 & 62.78 & 15.62 & 21.60 \\
\hline Hybrid Model 2 w/o Refinement & 1.46 & 12.73 & 62.72 & 16.54 & 20.74 \\
\hline Developed Hybrid Model 1 & 1.11 & 10.24 & 70.59 & 10.34 & 19.07 \\
\hline Developed Hybrid Model 2 & 1.15 & 10.12 & 74.50 & 3.33 & 22.17 \\
\hline
\end{tabular}

Tables 4-4a and 4-4b present the accuracy and reliability of various off-line estimation methods for simulated incident scenario 1 from 7:00 A.M. to 9:00 A.M. and from 7:30 A.M. to 8:30 A.M., respectively. As stated before, the off-line estimation can 
be used as a basis for training travel time prediction algorithms. Thus, the examination of off-line estimation results can give an indication of whether travel time prediction has the potential to improve the accuracy and reliability of the calculated travel time. In addition, off-line travel time estimation is important for planning studies. As shown in both tables, for off-line applications, the Minimum Speed method has a relatively better performance than other speed-based methods. Again, the developed hybrid models have higher accuracy and reliability than the other methods. The comparison between the results in Table 4-3b and Table 4-4b shows that both the on-line methods and off-line methods have similar performance except that the off-line Minimum Speed method and the developed off-line hybrid models perform better than their on-line versions, as illustrated in Figure 4-4.

Table 4-4a Accuracy and Reliability of Tested Off-Line Travel Time Estimation Methods for Simulated Incident Scenario 1 between 7:00 A.M. and 9:00 A.M.

\begin{tabular}{|l|c|c|c|c|c|}
\hline Method & $\begin{array}{c}\text { MAE } \\
\text { (Min.) }\end{array}$ & $\begin{array}{c}\text { MAPE } \\
\mathbf{( \% )}\end{array}$ & $\begin{array}{c}\text { Reliability } \\
\mathbf{( \% )}\end{array}$ & \% Early & \% Late \\
\hline Point-to-Point Method & 1.09 & 9.28 & 80.65 & 0 & 19.35 \\
\hline Mid-Point Method & 0.94 & 7.74 & 83.63 & 0 & 16.37 \\
\hline Minimum Speed Method & 0.44 & 4.45 & 91.30 & 4.76 & 3.94 \\
\hline Average Speed Method & 1.25 & 9.99 & 80.07 & 0 & 19.93 \\
\hline Minnesota Method & 1.04 & 8.51 & 79.99 & 0 & 20.01 \\
\hline Linear Speed Method & 1.17 & 9.41 & 79.39 & 0 & 20.61 \\
\hline Constant Acceleration Method & 1.28 & 10.19 & 79.39 & 0 & 20.61 \\
\hline Developed Hybrid Model 1 & 0.42 & 3.84 & 94.17 & 1.18 & 4.65 \\
\hline Developed Hybrid Model 2 & 0.42 & 3.86 & 91.65 & 1.59 & 6.76 \\
\hline
\end{tabular}


Table 4-4b Accuracy and Reliability of Tested Off-Line Travel Time Estimation Methods for Simulated Incident Scenario 1 between 7:30 A.M. and 8:30 A.M.

\begin{tabular}{|l|c|c|c|c|c|}
\hline Method & $\begin{array}{c}\text { MAE } \\
\text { (Min.) }\end{array}$ & $\begin{array}{c}\text { MAPE } \\
(\mathbf{\%})\end{array}$ & $\begin{array}{c}\text { Reliability } \\
(\mathbf{\%})\end{array}$ & \% Early & \% Late \\
\hline Point-to-Point Method & 2.06 & 16.76 & 59.39 & 0 & 40.61 \\
\hline Mid-Point Method & 1.83 & 14.82 & 65.65 & 0 & 34.35 \\
\hline Minimum Speed Method & 0.75 & 6.82 & 87.02 & 4.71 & 8.27 \\
\hline Average Speed Method & 2.45 & 19.24 & 58.19 & 0 & 41.82 \\
\hline Minnesota Method & 2.04 & 16.32 & 58.01 & 0 & 41.99 \\
\hline Linear Speed Method & 2.29 & 18.10 & 56.75 & 0 & 43.25 \\
\hline Constant Acceleration Method & 2.50 & 19.65 & 56.75 & 0 & 43.25 \\
\hline Developed Hybrid Model 1 & 0.80 & 7.02 & 87.77 & 2.47 & 9.76 \\
\hline Developed Hybrid Model 2 & 0.79 & 7.05 & 82.48 & 3.33 & 14.19 \\
\hline
\end{tabular}

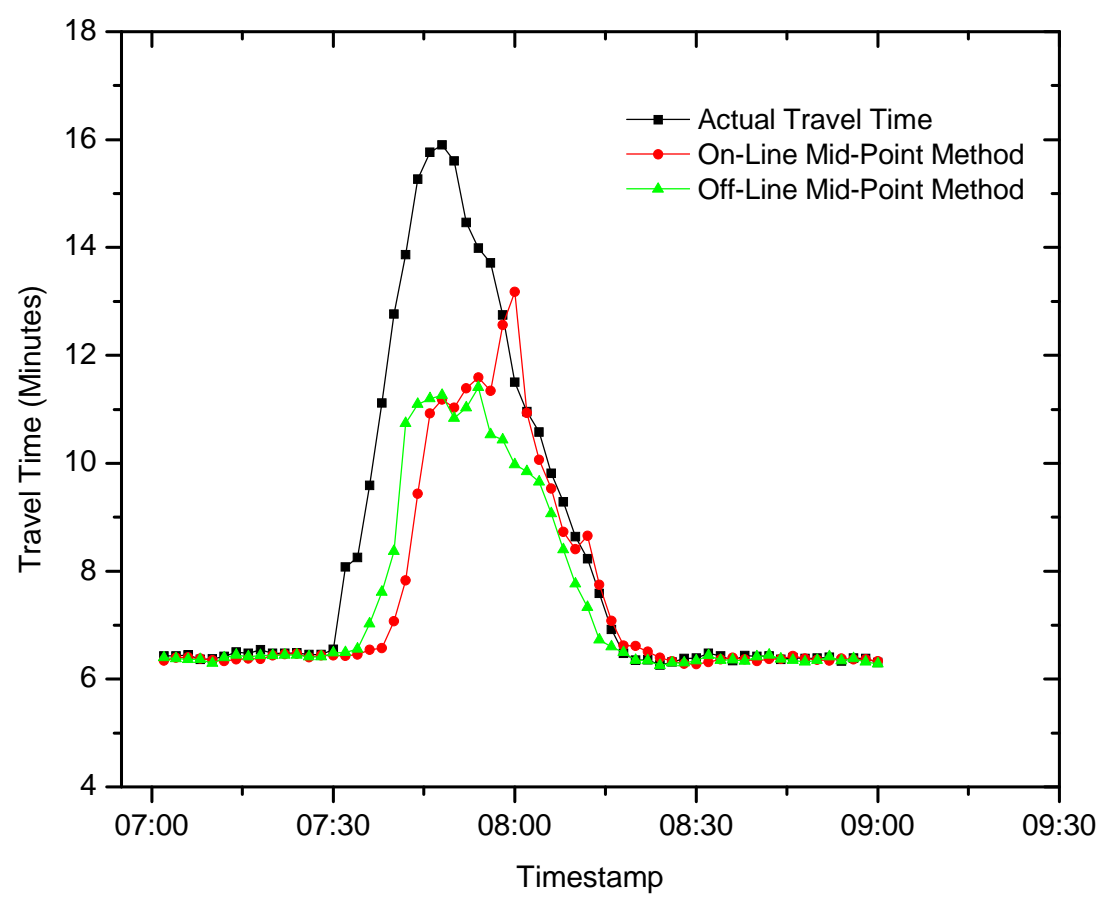

(a) Mid-Point Method

Figure 4-4 Comparison of On-Line and Off-Line Estimation Methods

(continued on next page) 


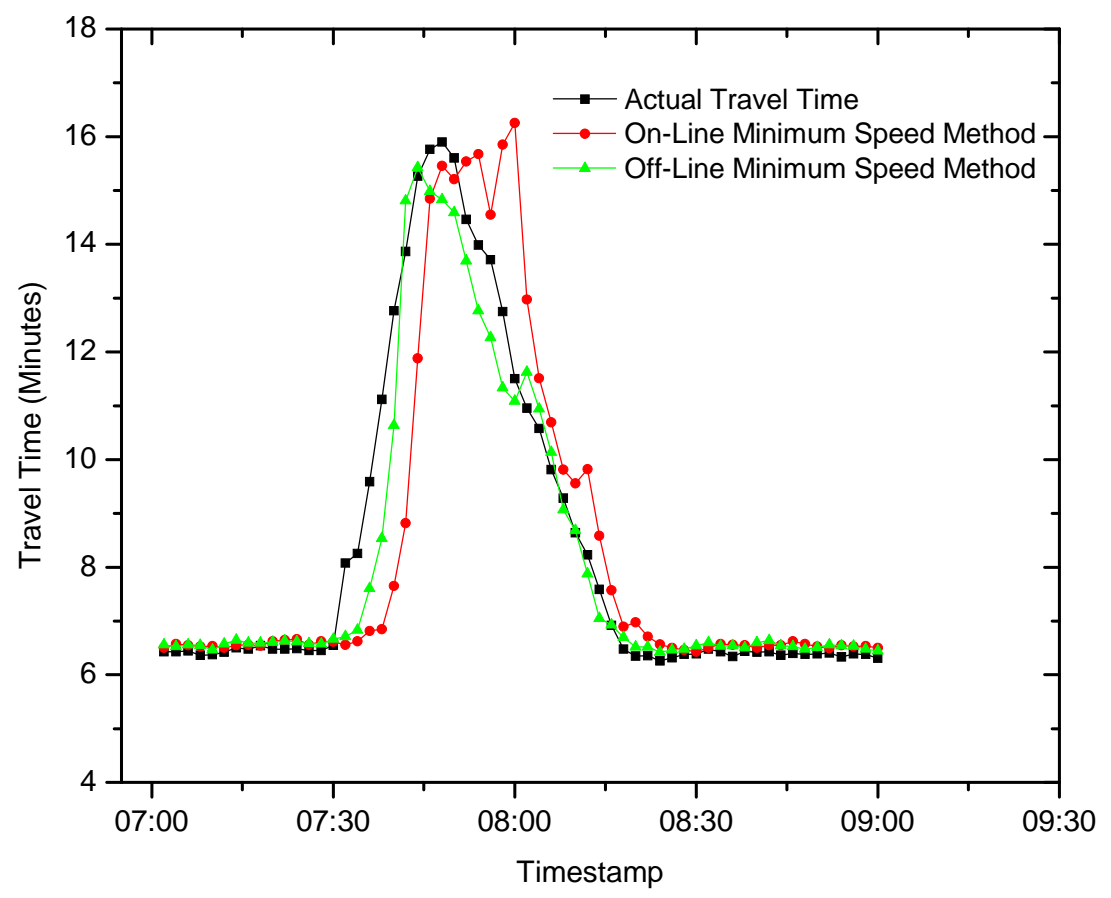

(b) Minimum Speed Method

Figure 4-4 Comparison of On-Line and Off-Line Estimation Methods

\subsubsection{Simulated Incident Scenario 2}

Figure 4-5 presents the results for on-line travel time estimation for Simulated Incident Scenario 2. Similar to the Simulated Incident Scenario 1, the incident attributes in Scenario 2 correspond to a real-world incident from the FDOT District 6 SunGuide incident management database. This one-lane blockage incident occurred at 7:23 A.M. After 35 minutes, the incident was moved to the shoulder and the blocked lane was open. The incident was completely cleared at 8:45 A.M., 82 minutes after it started. This incident is more severe than Scenario 1, as it has a longer duration and the capacity drop due to the incident is higher by about 35\%. The arrival of fire trucks at this incident location appears to have a higher impact on the capacity compared to Scenario 1. Figure 4-5(a) shows that the estimation results from various on-line methods. To show 
the results more clearly, Figure 4-5(b) presents the same results but only for the SunGuide method, Minimum Speed method, and on-line Hybrid Models. As shown in these two figures, the travel time estimated using various on-line methods are not satisfactory in this incident scenario unless a refinement is applied to the hybrid models to account for the front shock wave recovery. The SunGuide method (i.e., Point-to-Point method with capped speed) underestimate the travel time during the queue forming stage, and overestimate the travel time at the end of lane blockage. A similar trend can be found for other methods. One reason for this is that these methods estimate the travel time based on the current traffic conditions, without capturing the dynamic changes in the queue length as the vehicles progress from the departure location to the destination. As shown in Figure 4-5(b), the refined on-line hybrid models overcome this shortcoming with the consideration of a front recovery shock wave. Figure 4-5(c) presents the corresponding off-line travel time estimation results. It is seen from this figure that the off-line estimation methods can avoid the unrealistic estimated peaking in travel time observed in the on-line methods at the later lane-blockage stage when not considering the front recovery shock wave. 


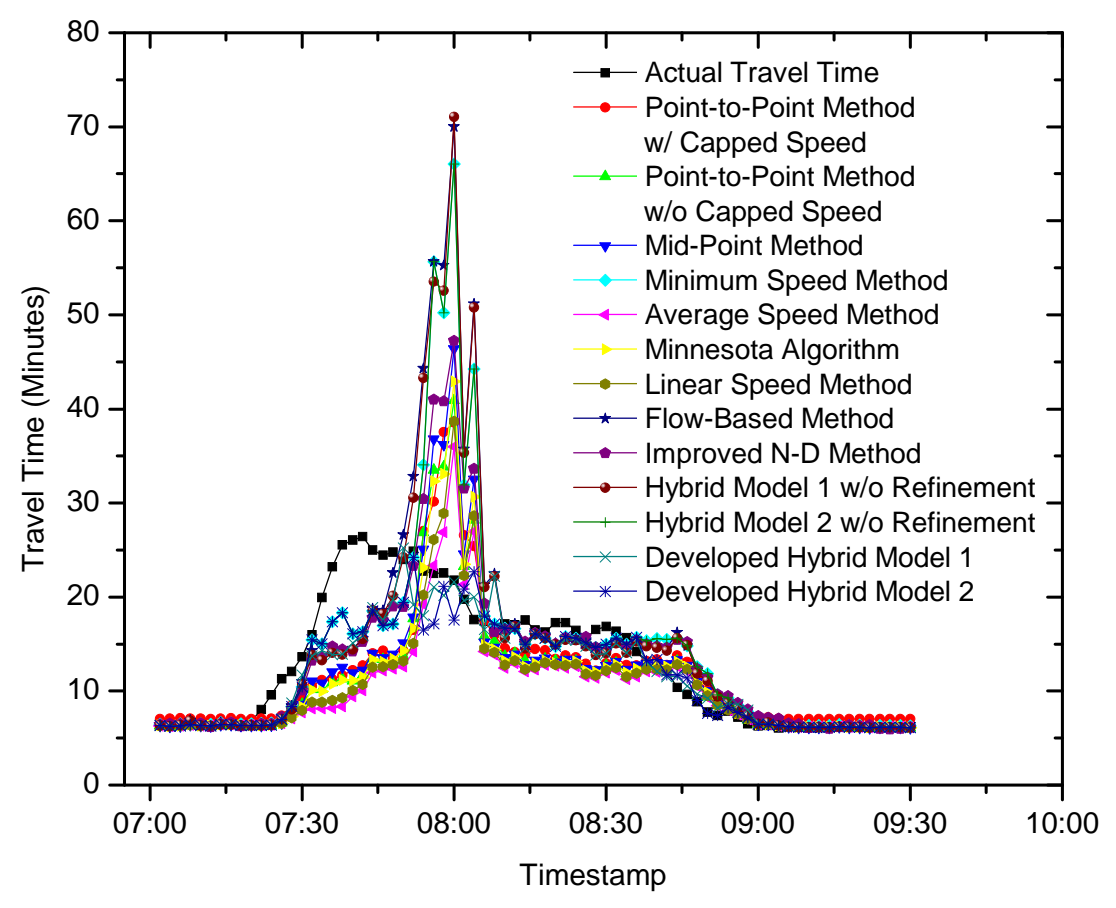

(a) On-Line Estimation Results

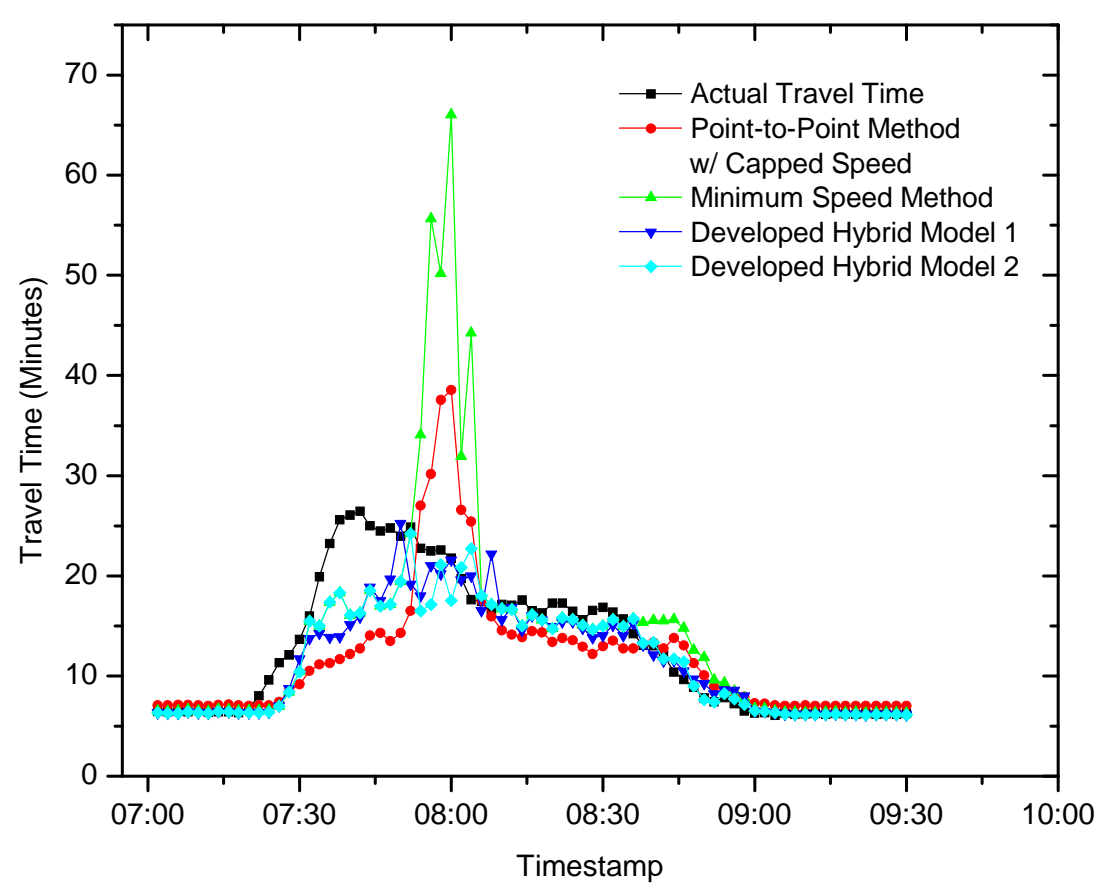

(b) On-Line Estimation Results

Figure 4-5 Estimated Travel Time for Simulated Incident Scenario 2

(continued on next page) 


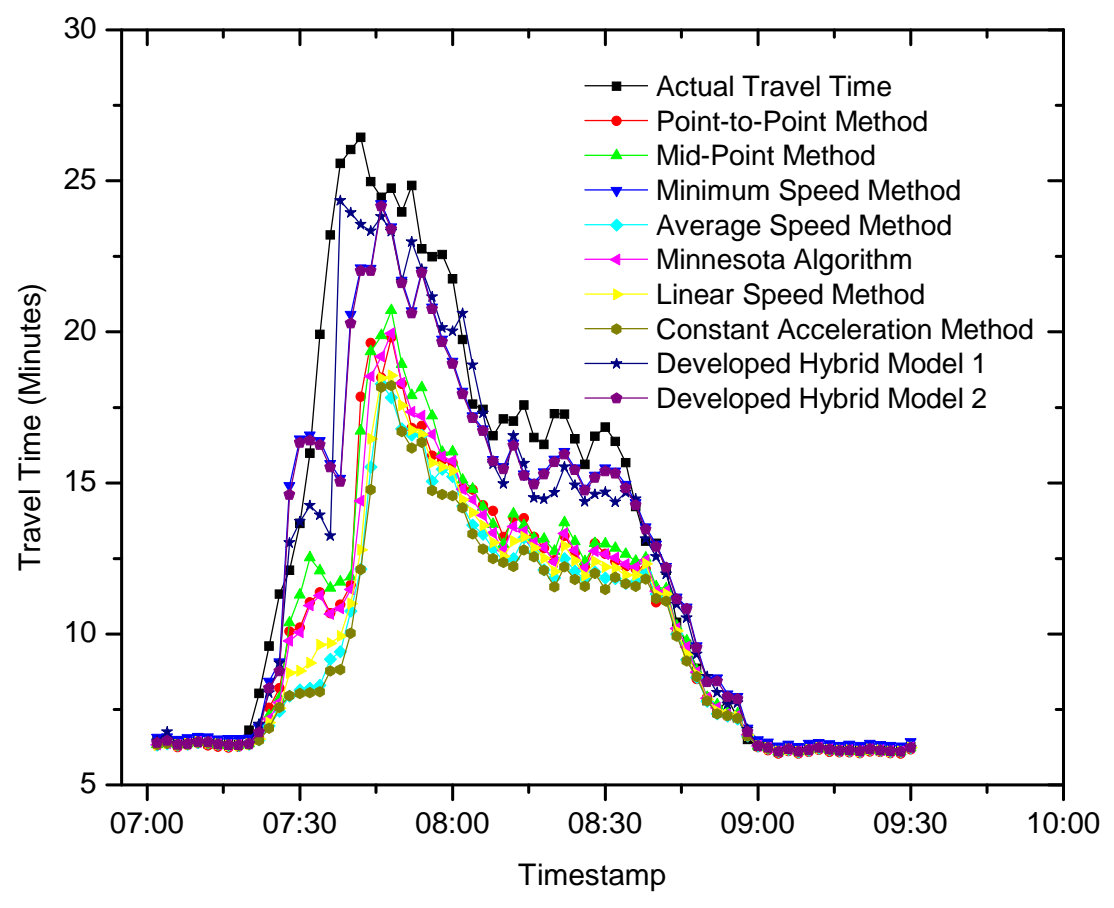

(c) Off-Line Estimation Results

\section{Figure 4-5 Estimated Travel Time for Simulated Incident Scenario 2}

Table 4-5 presents the performance of on-line and off-line travel time estimation methods for Simulated Incident Scenario 2. As shown in Table 4-5, the travel time produced by the SunGuide method with capped speed has a MAE of 3.61 minutes, a MAPE of $22 \%$, and a reliability of $55 \%$. About $18 \%$ of the vehicles arrive at the destination earlier than the posted travel time range and $26 \%$ of vehicles arrive late. Without capping the speed, the results are a little bit better, but still are not good. Table 4-5 also reveals that the on-line hybrid models do not perform well without the refinements. However, with the refinements, the developed on-line hybrid models produce much better results when compared to the other methods. The comparison between these two on-line hybrid models shows that Model 2 has lower errors and higher 
reliability than Model 1 under this scenario. The reason for the difference in performance of the travel time estimation methods between Incidents 1 and 2 is that Incident 2 has a more severe capacity constraint. Removing this constraint during the incident clearance stage resulted in a higher and faster impact on the experienced travel time. Thus, not accounting for the recovery shock wave has a higher impact in Incident 2 compared to Incident 1.

Table 4-5 Accuracy and Reliability of Tested On-Line Travel Time Estimation Methods for Simulated Incident Scenario 2

\begin{tabular}{|l|c|c|c|c|c|}
\hline Method & $\begin{array}{c}\text { MAE } \\
\text { (Min.) }\end{array}$ & $\begin{array}{c}\text { MAPE } \\
(\mathbf{\%})\end{array}$ & $\begin{array}{c}\text { Reliability } \\
(\%)\end{array}$ & $\begin{array}{c}\text { \% } \\
\text { Early }\end{array}$ & \% Late \\
\hline Point-to-Point Method w/ Capped Speed & 3.61 & 22.27 & 55.36 & 18.37 & 26.27 \\
\hline Point-to-Point Method w/o Capped Speed & 3.56 & 19.57 & 61.27 & 8.66 & 30.07 \\
\hline Mid-point Method & 3.71 & 20.31 & 61.11 & 9.61 & 29.28 \\
\hline Minimum Speed Method & 3.88 & 22.28 & 59.53 & 21.59 & 18.88 \\
\hline Average Speed Method & 3.74 & 20.36 & 59.97 & 6.60 & 33.43 \\
\hline Minnesota Method & 3.68 & 20.17 & 60.22 & 9.45 & 30.33 \\
\hline Linear Speed Method & 3.70 & 20.23 & 60.10 & 7.63 & 32.27 \\
\hline Flow-Based Method & 4.49 & 25.04 & 60.46 & 20.32 & 19.22 \\
\hline Improved N-D Method & 3.32 & 19.92 & 59.61 & 20.52 & 19.87 \\
\hline Hybrid Model 1 w/o Refinement & 4.42 & 24.47 & 62.87 & 17.34 & 19.79 \\
\hline Hybrid Model 2 w/o Refinement & 3.84 & 21.66 & 60.87 & 18.94 & 20.19 \\
\hline Developed Hybrid Model 1 & 1.89 & 11.08 & 67.46 & 10.50 & 22.04 \\
\hline Developed Hybrid Model 2 & 1.75 & 9.82 & 70.03 & 7.71 & 22.26 \\
\hline
\end{tabular}

Table 4-6 lists the performances of the off-line estimation methods for the Simulated Incident Scenario 2. Compared to the results of Table 4-5, the off-line estimation methods perform much better than the on-line estimations. For example, the MAE, MAPE, and reliability are 3.71 minutes, 20\%, and 61\%, respectively for the on-line Mid-Point method; and 2.51 minutes, 13\%, and 72\%, respectively for the off-line counterpart. Again, the off-line Minimum Speed and the hybrid models produce satisfactory results under this scenario as shown in Table 4-6. The results in Table 4-6 
indicate that travel time prediction could be beneficial for incident conditions, particularly more severe incidents with long incident durations.

Table 4-6 Accuracy and Reliability of Tested Off-Line Travel Time Estimation Methods for Simulated Incident Scenario 2

\begin{tabular}{|l|c|c|c|c|c|}
\hline Method & $\begin{array}{c}\text { MAE } \\
\text { (Min.) }\end{array}$ & $\begin{array}{c}\text { MAPE } \\
(\mathbf{\%})\end{array}$ & $\begin{array}{c}\text { Reliability } \\
(\mathbf{\%})\end{array}$ & \% Early & \% Late \\
\hline Point-to-Point Method & 2.73 & 14.75 & 70.60 & 1.67 & 27.72 \\
\hline Mid-Point Method & 2.51 & 13.37 & 72.32 & 2.15 & 25.54 \\
\hline Minimum Speed Method & 1.21 & 7.35 & 73.68 & 11.49 & 14.83 \\
\hline Average Speed Method & 3.31 & 17.62 & 65.16 & 0.99 & 33.85 \\
\hline Minnesota Method & 2.77 & 14.74 & 69.41 & 2.04 & 28.55 \\
\hline Linear Speed Method & 3.10 & 16.47 & 67.22 & 0.99 & 31.79 \\
\hline Constant Acceleration Method & 3.43 & 18.23 & 64.08 & 0.99 & 34.93 \\
\hline Developed Hybrid Model 1 & 1.03 & 6.28 & 76.69 & 8.90 & 14.41 \\
\hline Developed Hybrid Model 2 & 1.21 & 7.07 & 75.60 & 9.05 & 15.36 \\
\hline
\end{tabular}

\subsection{Comparison Based on Real-world Data}

In addition to the use of simulation, further assessment of travel time estimation methods was made using real-world data. The actual travel time was collected using videos from CCTV cameras deployed along the study corridor by matching the vehicles passing the field of view of one CCTV camera location to those passing the field of view of another CCTV camera location. The results for one congested case and one uncongested case are presented in this section. Both investigated cases represent recurrent conditions (no incident conditions). For the uncongested case, the travel times between two detector stations, DS-1515E and DS-1545E (a distance of about 5.02 miles), were collected for the midday period on December 2, 2008. For the congested case, the travel times between detector stations DS-1519E and DS-1545E (a distance of about 4.58 miles) were collected for the morning peak period on March 10, 2010. 
Figure 4-6 presents the travel time estimation results for the two cases mentioned above. It should be mentioned that the congestion level in the congested case is significantly lower than the congestion level resulting from the incident scenarios explored in the simulation models and discussed in Section 4.3. Figures 4-6(a) and Figure 4-6(b) show the estimation results for uncongested conditions during the Midday period. It is seen from these figures that although the travel times produced by the Point-to-Point method with capped speed overestimates the travel time due to the capped speed, the difference between the estimated travel times by the SunGuide method and the actual travel time is small. The travel time estimates obtained using the speed-based methods as well as the developed models were also very close when the traffic is not congested. Compared to the other methods, the Minimum Speed method slightly overestimated the travel time, and the results from the flow-based method and improved N-D method experienced more fluctuations.

For the congested case, the results shown in Figures 4-6(c) and 4-6(d) indicate that except for the Minimum Speed method, all of the other speed-based methods, including the Point-to-Point method with capped speed, underestimated the travel time under congested traffic conditions. This was true for both the on-line and off-line applications. The flow-based method and the improved N-D method performed better. The developed hybrid models also produced travel times that were also close to the actual travel times. 


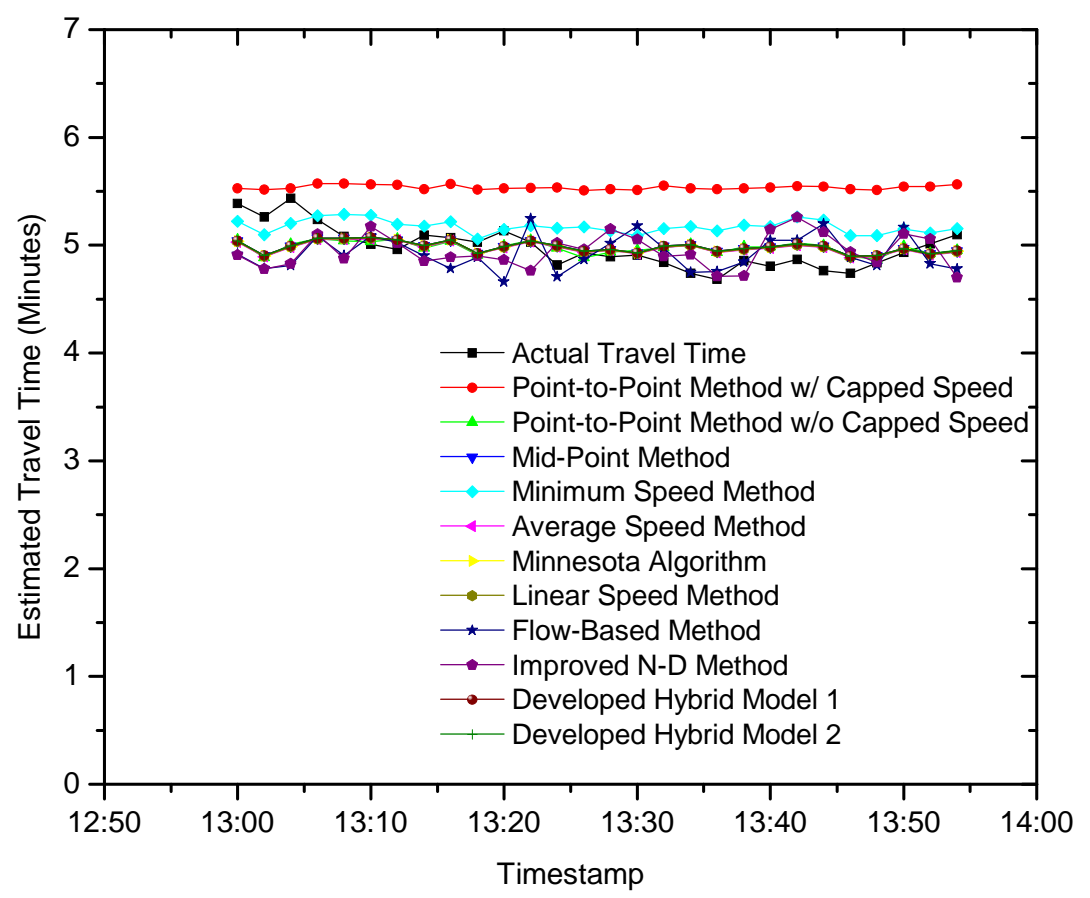

(a) On-Line Estimation Results during Midday on Dec. 2, 2008

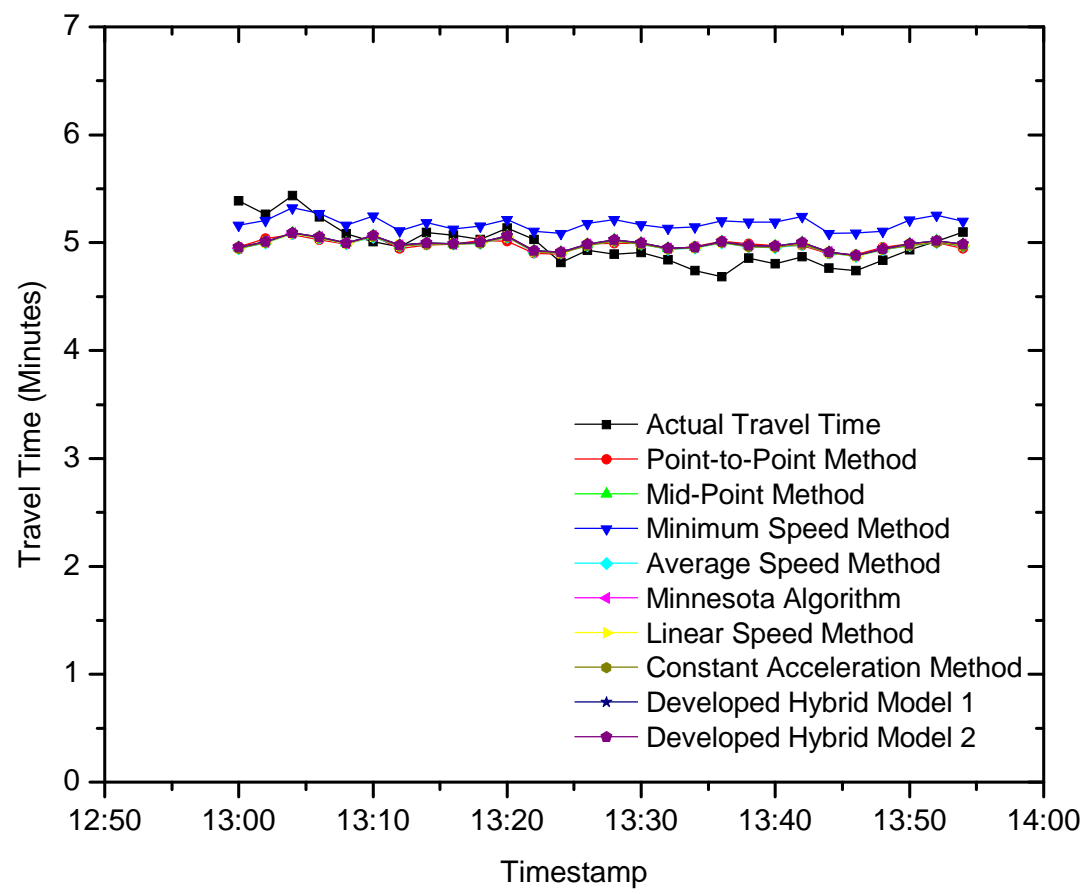

(b) Off-Line Estimation Results during Midday on Dec. 2, 2008

Figure 4-6 Estimated Travel Time for Real-world Case

(continued on next page) 


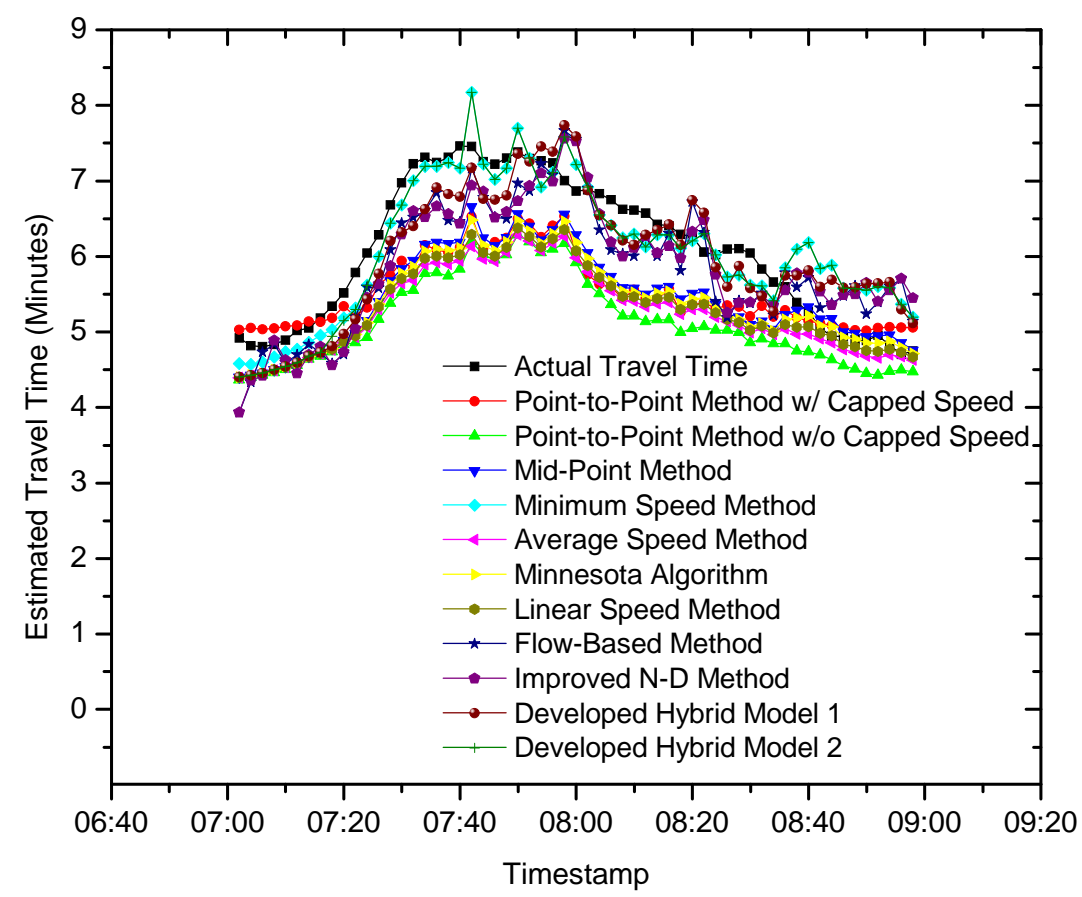

(c) On-Line Estimation Results during Morning Peak Period on Mar. 10, 2010

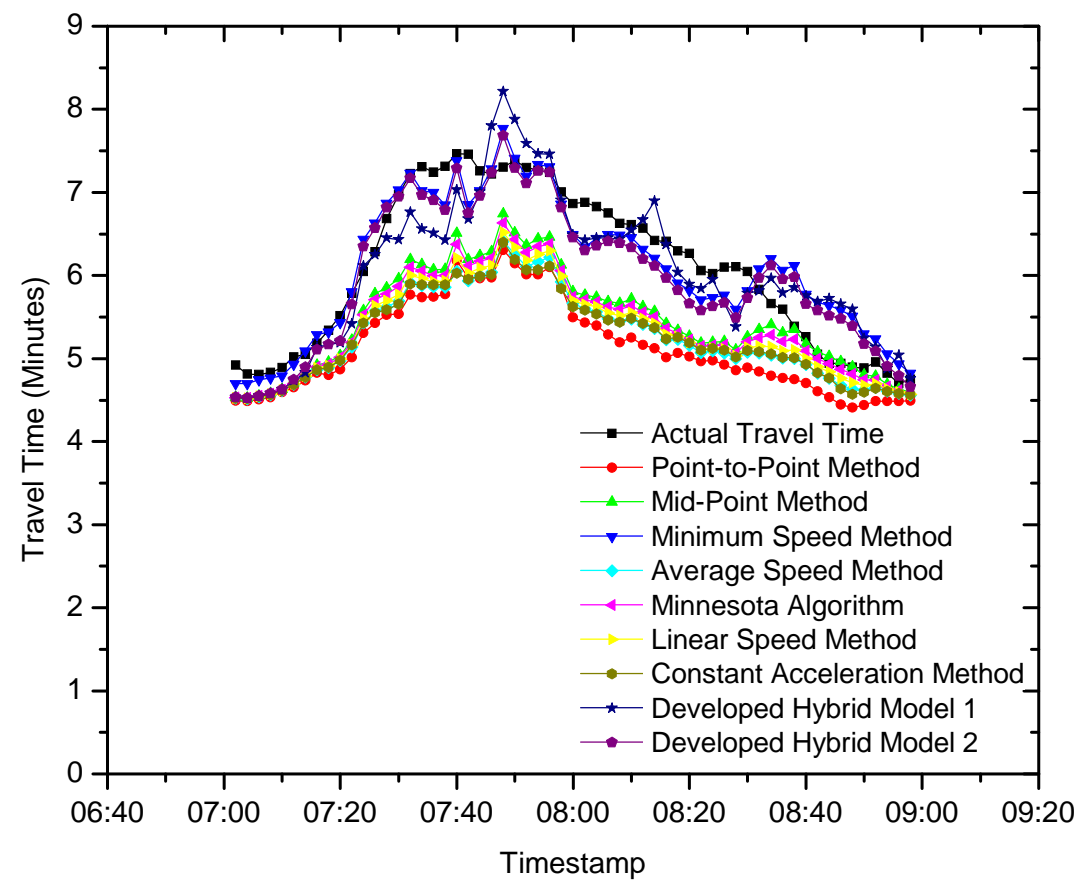

(d) Off-Line Estimation Results during Morning Peak Period on Mar. 10, 2010

Figure 4-6 Estimated Travel Time for Real-world Case 
Since not all individual vehicle travel times can be collected and the actual travel time distribution is unknown when using real-world point traffic detector data in the analysis, the reliability of the estimated travel time cannot be calculated using data that was produced based on simulation, as discussed in previous sections. Thus, only the accuracy performance measures are presented in Tables 4-7 and 4-8. As shown in Table 4-7, the performance of various travel time estimation methods during uncongested conditions (Case 1) are similar to those obtained from simulation. The Minimum Speed method, flow-based method, and the Improved N-D method were slightly less accurate than other methods for uncongested conditions. For congested conditions (Cases 2), the Minimum Speed method and Hybrid Model 2 perform the best among the tested methods. The traffic flow method and Hybrid Model 1 also perform relatively well compared to other methods. A comparison of the results from the off-line methods with those from the on-line methods indicates that the off-line estimation improved the travel time estimation slightly.

Table 4-7 Accuracy of Tested On-Line Travel Time Estimation Methods for Real-world Cases

\begin{tabular}{|l|c|c|c|c|}
\hline \multirow{2}{*}{ Method } & \multicolumn{3}{|l|}{ Case 1 (Uncongested) } & \multicolumn{2}{l|}{ Case 2 (Congested) } \\
\cline { 2 - 5 } & $\begin{array}{c}\text { MAE } \\
\text { (Min.) }\end{array}$ & $\begin{array}{c}\text { MAPE } \\
\mathbf{( \% )}\end{array}$ & $\begin{array}{c}\text { MAE } \\
\text { (Min.) }\end{array}$ & $\begin{array}{c}\text { MAPE } \\
\mathbf{( \% )}\end{array}$ \\
\hline Point-to-Point Method w/ Capped Speed & 0.56 & 11.32 & 0.65 & 9.95 \\
\hline Point-to-Point Method w/o Capped Speed & 0.14 & 2.81 & 0.94 & 14.78 \\
\hline Mid-point Method & 0.14 & 2.87 & 0.66 & 10.20 \\
\hline Minimum Speed Method & 0.23 & 4.71 & 0.37 & 6.31 \\
\hline Average Speed Method & 0.14 & 2.83 & 0.81 & 12.66 \\
\hline Minnesota Method & 0.14 & 2.86 & 0.70 & 10.86 \\
\hline Linear Speed Method & 0.14 & 2.84 & 0.76 & 11.76 \\
\hline Flow-Based Method & 0.18 & 3.56 & 0.53 & 8.79 \\
\hline Improved N-D Method & 0.20 & 3.90 & 0.54 & 9.03 \\
\hline Developed Hybrid Model 1 & 0.14 & 2.87 & 0.46 & 7.75 \\
\hline Developed Hybrid Model 2 & 0.14 & 2.87 & 0.39 & 6.80 \\
\hline
\end{tabular}


Table 4-8 Accuracy of Tested Off-Line Travel Time Estimation Methods for Real-world Cases

\begin{tabular}{|l|c|c|c|c|}
\hline \multirow{2}{*}{ Method } & \multicolumn{2}{|c|}{ Case 1 (Uncongested) } & \multicolumn{2}{c|}{ Case 2 (Congested) } \\
\cline { 2 - 5 } & $\begin{array}{c}\text { MAE } \\
\text { (Min.) }\end{array}$ & $\begin{array}{c}\text { MAPE } \\
\mathbf{( \% )}\end{array}$ & $\begin{array}{c}\text { MAE } \\
\text { (Min.) }\end{array}$ & $\begin{array}{c}\text { MAPE } \\
\mathbf{( \% )}\end{array}$ \\
\hline Point-to-Point Method & 0.14 & 2.71 & 0.94 & 14.75 \\
\hline Mid-point Method & 0.14 & 2.70 & 0.63 & 9.68 \\
\hline Minimum Speed Method & 0.23 & 4.67 & 0.33 & 5.40 \\
\hline Average Speed Method & 0.13 & 2.66 & 0.81 & 12.55 \\
\hline Minnesota Method & 0.14 & 2.68 & 0.69 & 10.61 \\
\hline Linear Speed Method & 0.13 & 2.67 & 0.75 & 11.65 \\
\hline Constant Acceleration Method & 0.13 & 2.66 & 0.82 & 12.63 \\
\hline Developed Hybrid Model 1 & 0.14 & 2.70 & 0.38 & 6.20 \\
\hline Developed Hybrid Model 2 & 0.10 & 2.70 & 0.35 & 5.74 \\
\hline
\end{tabular}

\subsection{Summary}

The developed hybrid travel time estimation models, as well as various speed-based methods and flow-based methods were evaluated in this chapter using both simulated and real-world travel time data. The performance measures in terms of accuracy and reliability were quantified. The results indicate that all tested methods perform reasonably well under uncongested traffic conditions. However, their performances vary with the increase in congestion level. The comparison with other estimation methods shows that the developed hybrid models perform well in all cases, but need refinements for on-line applications in the case of incident conditions particularly during incidents with a sharp drop in capacity. The refinement considers the dynamics of queue propagation and clearance, and requires the prediction of when the incident will be cleared. Further comparison between the on-line and off-line travel time estimation results reveals that off-line methods perform significantly better only during fast 
changing congested conditions such as during incidents. For uncongested conditions, the off-line methods produce results that are almost as good as their on-line counterparts. 


\section{CHAPTER 5}

\section{IMPACTS OF INFLUENTIAL FACTORS}

In addition to the impacts of the estimation algorithms on the quality of the estimations, factors such as the impacts of data preprocessing procedures, detector errors, detector spacing, and travel time posting strategies are also expected to affect the accuracy and reliability of travel time estimation. Therefore, the impacts of these factors on the on-line travel time estimation are investigated in this study. The results presented in this chapter are based on using the Hybrid Model 2 as an example, since the impacts of these factors on all other methods generally show the same trend as this hybrid model. The analysis results of the other estimation methods can be found in Appendix A. Note that Hybrid Models 1 and 2 in these tables refer to the refined on-line hybrid models developed in this study.

\subsection{Data Preprocessing}

Data preprocessing includes data filtering, data smoothing, data aggregation, and data imputation. This study investigates and compares the impacts of different methods to perform these steps on travel time estimation, as described below.

\subsubsection{Data Smoothing}

In this section, two different smoothing methods are compared: the simple moving average (which is the method used in SunGuide) and the exponential moving average. The Simulated Incident Scenario 1, described in Section 4.3.2, is used to test the impacts of data smoothing methods on travel time estimation. In this scenario, the estimated 
travel time is updated every 2 minutes, and the estimation performance is calculated for the time period 7:30 A.M. - 8:30 A.M. Table 5-1 presents the estimation results with these two types of smoothing methods for the on-line Hybrid Model 2. As shown in Table 5-1, for the simple moving average method, the estimation errors generally increase with the increase in the value of rolling period. As mentioned in the previous section, the rolling period is a parameter that controls the moving window size for the determination of number of data points to be used in the moving average. When a large rolling period is used, more historical information is included in the travel time estimation, which can dilute changes in traffic conditions such as queue length changes, resulting in higher estimation errors in fast changing situations like incident scenarios. It can also be seen from this table that a smaller rolling period will achieve better reliability in travel time estimation for incident conditions.

The travel time estimation results obtained using the exponential moving average are also presented in Table 5-1. The smoothing factor in the exponential moving average method can have any value between 0 and 1 . A higher value of this smoothing factor reduces the effects of older observations faster. Comparing the results with those calculated from the simple moving average indicates that for Hybrid Model 2 and the investigated incident scenario, the exponential moving average method produces significantly more accurate and reliable results than the simple moving average method, since the exponential moving average method can give more weight to the latest data in the smoothing. A smoothing factor of 0.4 is sufficient to produce good results. It should be mentioned that these results are based on a simulation analysis that assumes $100 \%$ detector measurement accuracy. 
Table 5-1 Accuracy and Reliability of Travel Time Estimation Using Different Smoothing Methods

\begin{tabular}{|c|c|c|c|c|c|c|c|}
\hline $\begin{array}{l}\text { Hybrid } \\
\text { Model } 2\end{array}$ & \multicolumn{2}{|c|}{ Factor } & $\begin{array}{l}\text { MAE } \\
\text { (Min.) }\end{array}$ & $\begin{array}{c}\text { MAPE } \\
(\%)\end{array}$ & $\begin{array}{c}\text { Reliability } \\
\text { (\%) }\end{array}$ & $\begin{array}{c}\% \\
\text { Early }\end{array}$ & $\%$ Late \\
\hline \multirow{5}{*}{$\begin{array}{l}\text { Simple } \\
\text { Moving } \\
\text { Average }\end{array}$} & \multirow{5}{*}{$\begin{array}{c}\text { Rolling } \\
\text { Period }\end{array}$} & 1-minute & 1.44 & 12.72 & 63.70 & 17.17 & 19.13 \\
\hline & & 2-minute & 1.59 & 13.86 & 61.45 & 16.72 & 21.83 \\
\hline & & 3-minute & 1.73 & 15.00 & 58.93 & 16.72 & 24.35 \\
\hline & & 4-minute & 1.89 & 16.29 & 56.23 & 19.01 & 24.76 \\
\hline & & 5-minute & 2.04 & 17.72 & 48.65 & 24.81 & 26.54 \\
\hline \multirow{5}{*}{$\begin{array}{l}\text { Exponential } \\
\text { Moving } \\
\text { Average }\end{array}$} & \multirow{5}{*}{$\begin{array}{c}\text { Smoothing } \\
\text { Factor }\end{array}$} & 0.2 & 1.49 & 12.51 & 69.21 & 3.33 & 27.46 \\
\hline & & 0.4 & 1.15 & 10.12 & 74.50 & 3.33 & 22.17 \\
\hline & & 0.6 & 1.03 & 9.28 & 73.92 & 4.36 & 21.71 \\
\hline & & 0.8 & 0.99 & 8.98 & 74.73 & 4.54 & 20.74 \\
\hline & & 1.0 & 1.09 & 9.60 & 73.23 & 4.48 & 22.29 \\
\hline
\end{tabular}

\subsubsection{Data Imputation}

To test the effects of different data imputation methods, $50 \%$ of the detector measurements were randomly removed for the Simulated Incident Scenario 1. As mentioned earlier in this chapter, data imputation can be conducted spatially and/or temporally, and the spatial imputation can be performed within a station or between stations. Therefore, different combinations of these imputation types were tested in this study including with or without within-station imputation for speed, with or without temporal imputation, and four different types of between-station imputations that are commonly used in practice. These four types are the following:

- Simple average,

- Linear interpolation,

- Linear interpolation for speed and occupancy but factor method for volume, and 
- Factor method for all traffic parameters, as mentioned in the data preprocessing section.

For the factor method, the factors are estimated based on data for all workdays in December, 2008. The results are presented in Table 5-2.

Table 5-2 Results of Different Data Imputation Methods

\begin{tabular}{|c|c|c|c|c|c|c|c|}
\hline $\begin{array}{l}\text { Hybrid } \\
\text { Model } 2 \\
\end{array}$ & \begin{tabular}{|l|} 
Temporal \\
Imputation
\end{tabular} & $\begin{array}{l}\text { Between-Station } \\
\text { Imputation }\end{array}$ & $\begin{array}{l}\text { MAE } \\
\text { (Min.) }\end{array}$ & $\begin{array}{c}\text { MAPE } \\
(\%)\end{array}$ & \begin{tabular}{|c|} 
Reliability \\
$(\%)$
\end{tabular} & $\begin{array}{c}\% \\
\text { Early }\end{array}$ & $\begin{array}{c}\% \\
\text { Late }\end{array}$ \\
\hline \multicolumn{3}{|c|}{ Hybrid Model 2 w/o missing data } & 1.15 & 10.12 & 74.50 & 3.33 & 22.17 \\
\hline \multirow{8}{*}{$\begin{array}{l}\text { w/o } \\
\text { Within- } \\
\text { Station } \\
\text { Imputation }\end{array}$} & \multirow{4}{*}{$\begin{array}{l}\text { w/o } \\
\text { Temporal } \\
\text { Imputation }\end{array}$} & Simple Average & 1.23 & 10.72 & 72.20 & 4.77 & 23.03 \\
\hline & & Linear Interpolation & 1.23 & 10.70 & 72.20 & 4.77 & 23.03 \\
\hline & & $\begin{array}{l}\text { Linear Interpolation } \\
\text { for S and } \mathrm{O} \text {, and } \\
\text { Factor for } \mathrm{V}\end{array}$ & 1.23 & 10.70 & 72.20 & 4.77 & 23.03 \\
\hline & & Factor Method & 1.24 & 10.77 & 71.97 & 4.77 & 23.26 \\
\hline & \multirow{4}{*}{\begin{tabular}{|l} 
\\
Average of \\
Temporal \\
and Spatial \\
Imputations
\end{tabular}} & Simple Average & 1.23 & 10.64 & 72.20 & 4.77 & 23.03 \\
\hline & & Linear Interpolation & 1.22 & 10.61 & 72.20 & 4.77 & 23.03 \\
\hline & & \begin{tabular}{|l|} 
Linear Interpolation \\
for S and $\mathrm{O}$, and \\
Factor for $\mathrm{V}$ \\
\end{tabular} & 1.22 & 10.61 & 72.20 & 4.77 & 23.03 \\
\hline & & Factor Method & 1.23 & 10.64 & 72.20 & 4.77 & 23.03 \\
\hline \multirow{8}{*}{$\begin{array}{l}\text { W/ } \\
\text { Within- } \\
\text { Station } \\
\text { Imputation }\end{array}$} & \multirow{4}{*}{$\begin{array}{l}\text { w/o } \\
\text { Temporal } \\
\text { Imputation }\end{array}$} & Simple Average & 1.20 & 10.48 & 73.58 & 4.77 & 21.65 \\
\hline & & Linear Interpolation & 1.20 & 10.48 & 73.58 & 4.77 & 21.65 \\
\hline & & $\begin{array}{l}\text { Linear Interpolation } \\
\text { for S and } \mathrm{O} \text {, and } \\
\text { Factor for } \mathrm{V} \\
\end{array}$ & 1.20 & 10.48 & 73.58 & 4.77 & 21.65 \\
\hline & & Factor Method & 1.20 & 10.48 & 73.58 & 4.77 & 21.65 \\
\hline & \multirow{4}{*}{$\begin{array}{l}\text { Average of } \\
\text { Temporal } \\
\text { and Spatial } \\
\text { Imputations }\end{array}$} & Simple Average & 1.20 & 10.48 & 73.58 & 4.77 & 21.65 \\
\hline & & Linear Interpolation & 1.20 & 10.48 & 73.58 & 4.77 & 21.65 \\
\hline & & $\begin{array}{l}\text { Linear Interpolation } \\
\text { for S and } \mathrm{O} \text {, and } \\
\text { Factor for } \mathrm{V} \\
\end{array}$ & 1.20 & 10.48 & 73.58 & 4.77 & 21.65 \\
\hline & & Factor Method & 1.20 & 10.48 & 73.58 & 4.77 & 21.65 \\
\hline
\end{tabular}

Note: $\mathrm{S}$ represents speed, $\mathrm{V}$ dictates volume count, and $\mathrm{O}$ is occupancy.

Table 5-2 shows the impacts of the data imputation methods on the performance of travel time estimation results. It should be mentioned that the performance 
calculation is based on the time period between 7:30 A.M. and 8:30 A.M, which is heavily impacted by the incident conditions. The results in this table show that the impact of randomly missing data when properly imputed is not high. As mentioned above $50 \%$ of the detector measurements were removed randomly. Slight differences in the accuracy of the resulting travel times can be observed from Table 5-2 for different imputation methods. Note that the rows with within-station imputation, simple average between stations, and without temporal imputation corresponds to the imputation method used in SunGuide software. Since travel time cannot be estimated when some of detector data are missing, the results presented in Table 5-2 cannot be compared to those without data imputation.

\subsection{Detector Errors}

Traffic data measured by point detectors include errors of different types:

- Intrinsic errors due to measurement noise that reflects detector inaccuracy,

- Systematic errors (for example, due to inadequate calibration or device inaccuracy), and

- Data missing due to incidental and/or structural failure resulting from temporary power outages or detector malfunctions.

The impacts of these three types of errors on travel time estimation performance are investigated in this study and discussed below.

\subsubsection{Intrinsic Errors}

Intrinsic errors are inherent to detectors and reflect their measurement inaccuracies. The magnitude of the intrinsic error in measuring a given variables depends on the detector 
type under consideration. For example, Electronic Integrated Systems Inc. (EIS), the vendor of the RTMS detectors, reported that for RTMS detectors from a side fire location; the errors are expected to be $10 \%$ in speed, $5 \%$ in volumes, $10 \%$ in long vehicle volumes, and 5\% in occupancy (EIS 2010). To investigate the impacts of such errors on travel time estimation, the detector data resulting from CORSIM were modified to emulate these types of errors. Similar to the study by Byon et al. (2009), a normal distribution was used to introduce the intrinsic errors in the simulated detector data of this study. The used normal distribution has a mean of zero, and a standard deviation determined by device measurement accuracy. As shown in Figure 5-1, 99.7\% of measurements are within 6 standard deviations of the mean detector error (zero) for a normal distribution. This assumption leads to the following equation:

$$
6 \sigma=2 X_{\text {typical }} \times E r r
$$

where $\sigma$ denotes the standard deviation, $X_{\text {typical }}$ is selected to be a common measurement value at the high end of each of the three basic variables (speed, volume, or occupancy), and Err is the corresponding measurement error. Measurement at the high end is selected such that the worst case standard deviation is accounted for. As an example, this investigation assumes that Err for speed measurement is 10\%; Err for volume measurement is 5\%, and Err for occupancy measurement is also 5\%. With the assumptions of $65 \mathrm{mph}, 1,700 \mathrm{veh} / \mathrm{hr}$, and 100\%, as respective typical speed, volume, and occupancy measurements, Equation 5-1 yields the following standard deviations values: $2.2 \mathrm{mph}$ for speed, $28.3 \mathrm{veh} / \mathrm{hr}$ for volume, and 1.7 for occupancy. Note that this is only one example. Depending on the local situation and specific devices used, the values of Err and $X_{\text {typical }}$ may vary but the same methodology can still be applied. 


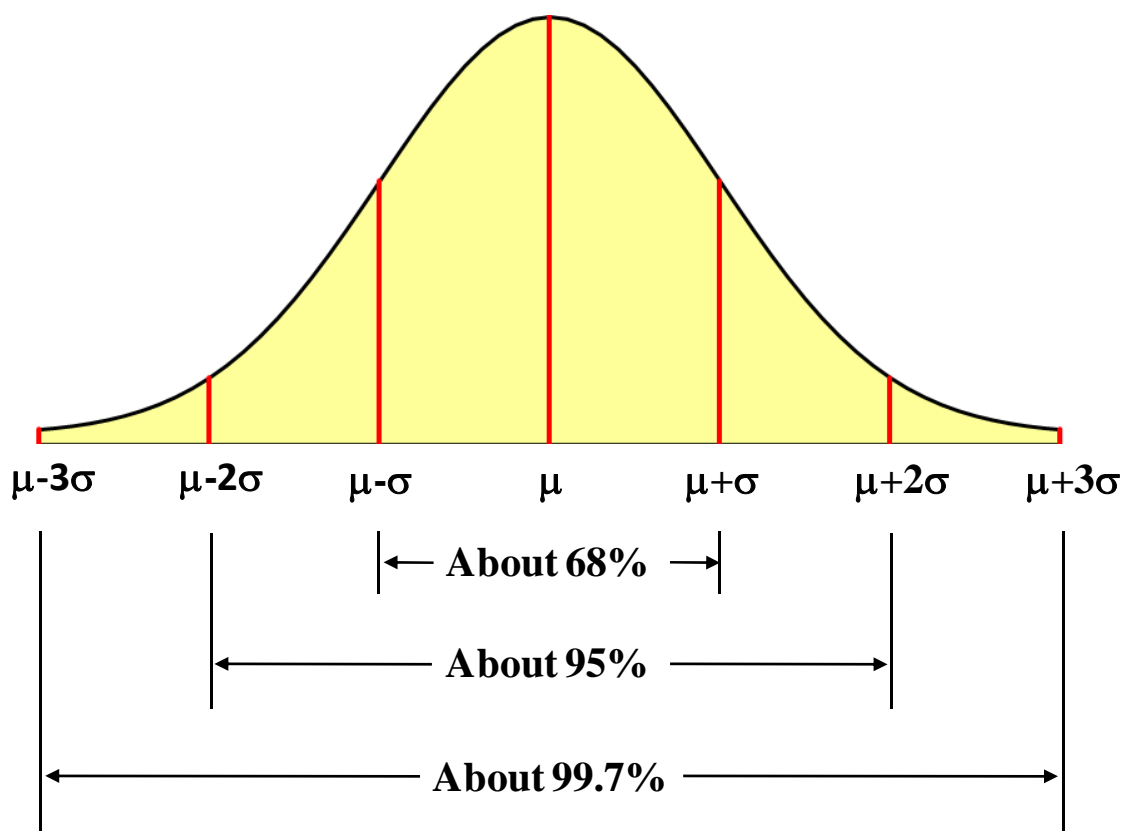

Figure 5-1 Illustration of Normal Distribution for Intrinsic Errors

In this study, the intrinsic errors were introduced in two simulated scenarios: an uncongested scenario and Incident Scenario 1. For each scenario, 10 random cases were generated and the results are presented in Table 5-3 for Hybrid Model 2 and in Tables A-5 and A-6 (in Appendix A) for other methods. Note that a number of 10 random cases satisfies the minimum sample size required at a 95\% confidence interval for normal distribution. As shown in Table 5-3, the performance of Hybrid Model 2 does not change much with the intrinsic errors introduced for the uncongested scenario. However, the performance is affected by the error for the incident scenario. It should be noted that the results presented in Table 5-3 are with data filtering and imputation. The impacts of intrinsic errors are expected to be higher without proper filtering and imputation and also with higher detector error. 
Table 5-3 Impacts of Intrinsic Errors on Travel Time Estimation Performance

\begin{tabular}{|c|c|c|c|c|c|c|c|}
\hline Hybrid Model 2 & \multicolumn{2}{|c|}{ Cases } & $\begin{array}{l}\text { MAE } \\
\text { (Min.) }\end{array}$ & $\begin{array}{c}\text { MAPE } \\
(\%)\end{array}$ & \begin{tabular}{|c|} 
Reliability \\
$(\%)$
\end{tabular} & $\begin{array}{c}\% \\
\text { Early }\end{array}$ & \% Late \\
\hline \multirow{4}{*}{$\begin{array}{l}\text { Simulated } \\
\text { Uncongested } \\
\text { Conditions }\end{array}$} & \multicolumn{2}{|c|}{ w/o Intrinsic Errors } & 0.082 & 1.31 & 100 & 0 & 0 \\
\hline & \multirow{3}{*}{$\begin{array}{c}\text { w/ } \\
\text { Intrinsic } \\
\text { Errors }\end{array}$} & Average & 0.083 & 1.33 & 100 & 0 & 0 \\
\hline & & Minimum & 0.081 & 1.30 & 100 & 0 & 0 \\
\hline & & Maximum & 0.085 & 1.36 & 100 & 0 & 0 \\
\hline \multirow{4}{*}{$\begin{array}{l}\text { Simulated } \\
\text { Incident } \\
\text { Conditions }\end{array}$} & \multicolumn{2}{|c|}{ w/o Intrinsic Errors } & 1.15 & 10.12 & 74.50 & 3.33 & 22.17 \\
\hline & \multirow{3}{*}{$\begin{array}{c}\text { w/ } \\
\text { Intrinsic } \\
\text { Errors }\end{array}$} & Average & 1.58 & 12.87 & 64.59 & 4.24 & 31.17 \\
\hline & & Minimum & 1.23 & 10.66 & 61.17 & 2.59 & 22.34 \\
\hline & & Maximum & 1.75 & 13.97 & 71.17 & 6.49 & 34.75 \\
\hline
\end{tabular}

\subsubsection{Systematic Errors}

Point detectors are not always well calibrated or have systematic underestimation or overestimation problems in measurements. These are referred to as systematic errors in the reported measurements. Examination of the detector measurements in this study showed that some detector stations always reported consistently high or consistently low values compared to the expected values based on adjacent detector measurements and traffic flow theory concepts. Figure 5-2 shows the average free flow speed based on the detector data reported at station, DS-1513E, for two time periods. As mentioned in Highway Capacity Manual (HCM) 2000, the free flow speed of a freeway segment is expected to prevail at flow rates between $0 \mathrm{pc} / \mathrm{h} / \mathrm{ln}$ and 1,200 pc/h/ln (HCM 2000). In this study, this definition was used to estimate the average free flow speed for the detector station with potential problems based on 5-minute aggregated detector data between 12:00 A.M. and 5:00 A.M. from December 1, 2008 to February 28, 2009. As shown in Figure 5-2, the data set is divided into two time periods. Time Period 1 is from December 1, 2008 to January 15, 2009 and Time Period 2 is from January 16, 2009 to February 28, 2009. It is seen from this figure that for Time Period 1, the resulting free 
flow speed for Lane 1 is close to $90 \mathrm{mph}$ and the free flow speed for Lane 2 and Lane 3 are higher than $90 \mathrm{mph}$. However, in Time Period 2, the average free flow speed for Lane 1 drops to $50 \mathrm{mph}$, while those for Lanes 2 and 3 are about $65 \mathrm{mph} .65 \mathrm{mph}$ seems to be a reasonable free flow speed based on the free flow speeds at an adjacent detection station. The $90 \mathrm{mph}$ and $50 \mathrm{mph}$ speed measurements seem to be due to systematic errors of about $+26.87 \mathrm{mph}$ and $-14.6 \mathrm{mph}$, respectively.

To investigate the impacts of systematic errors on travel time estimation results, different scenarios were created in this study by introducing systematic errors to the error-free simulated detector data for uncongested scenario and Incident Scenario 1, as shown below in Table 5-4. Note that in each scenario having more than one detection station with a failure, the distances between the stations with the problem are selected to be equal.

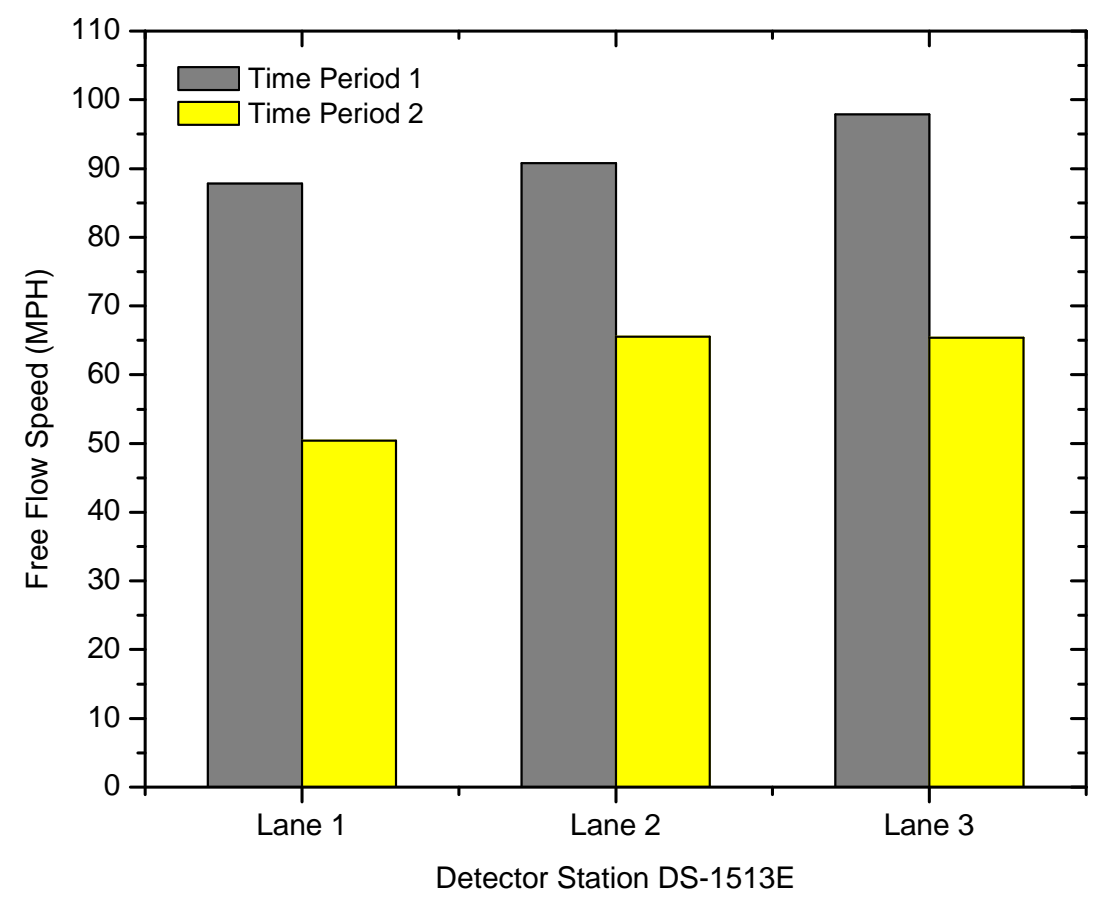

Figure 5-2 Estimated Free Flow Speed for Detection Station DS-1513E 
Table 5-4 Tested Scenarios for Systematic Errors

\begin{tabular}{|c|l|l|c|}
\hline Case No. & Detector Station & $\begin{array}{l}\text { Lanes with } \\
\text { Systematic Errors }\end{array}$ & $\begin{array}{c}\text { Systematic Error in Speed } \\
\text { (“+”: increase; } \\
\text { “_”: decrease) }\end{array}$ \\
\hline 1 & DS-1529E & All Lanes & $+26.87 \mathrm{mph}$ \\
\hline 2 & DS-1509E and DS-1529E & All Lanes & $+26.87 \mathrm{mph}$ \\
\hline 3 & $\begin{array}{l}\text { DS-1509E, DS-1523E and } \\
\text { DS-1535E }\end{array}$ & All Lanes & $+26.87 \mathrm{mph}$ \\
\hline 4 & $\begin{array}{l}\text { DS-1509E, DS-1521E, } \\
\text { DS-1529E and DS-1539E }\end{array}$ & Lane 1 & $+26.87 \mathrm{mph}$ \\
\hline 5 & $\begin{array}{l}\text { DS-1509E, DS-1521E, } \\
\text { DS-1529E and DS-1539E }\end{array}$ & Lane 1 and Lane 2 & $+26.87 \mathrm{mph}$ \\
\hline 6 & $\begin{array}{l}\text { DS-1509E, DS-1521E, } \\
\text { DS-1529E and DS-1539E }\end{array}$ & All Lanes & $+26.87 \mathrm{mph}$ \\
\hline 7 & DS-1529E & All Lanes & $-14.60 \mathrm{mph}$ \\
\hline 8 & DS-1509E and DS-1529E & All Lanes & $-14.60 \mathrm{mph}$ \\
\hline 9 & $\begin{array}{l}\text { DS-1509E, DS-1523E and } \\
\text { DS-1535E }\end{array}$ & All Lanes & $-14.60 \mathrm{mph}$ \\
\hline 10 & $\begin{array}{l}\text { DS-1509E, DS-1521E, } \\
\text { DS-1529E and DS-1539E }\end{array}$ & Lane 1 & $-14.60 \mathrm{mph}$ \\
\hline 11 & $\begin{array}{l}\text { DS-1509E, DS-1521E, } \\
\text { DS-1529E and DS-1539E }\end{array}$ & Lane 1 and Lane 2 & $-14.60 \mathrm{mph}$ \\
\hline 12 & $\begin{array}{l}\text { DS-1509E, DS-1521E, } \\
\text { DS-1529E and DS-1539E }\end{array}$ & All Lanes & $-14.60 \mathrm{mph}$ \\
\hline
\end{tabular}

Table 5-5 presents the performance of Hybrid Model 2 for the simulated uncongested and incident conditions when systematic errors are introduced. It is shown in Table 5-5a that with larger systematic errors, both accuracy and reliability are reduced. Comparing the results with and without data filtering indicates that the developed data filtering procedures can slightly improve the estimation performance for systematic high speed errors, but not for systematic low speed errors, since it is difficult to differentiate the systematic low speed from the drops in speeds due to congestion. Tables 5-5b presents similar results but for incident conditions. Again, the results in this table show that the data filtering procedures may help to improve the performance of the estimation method. 
Table 5-5a Impacts of Systematic Errors on Travel Time Estimation Performance for Simulated Uncongested Conditions

\begin{tabular}{|c|c|c|c|c|c|c|c|c|}
\hline \multirow[b]{2}{*}{$\begin{array}{l}\text { Hybrid } \\
\text { Model } 2\end{array}$} & \multicolumn{3}{|c|}{ Cases } & \multirow[b]{2}{*}{$\begin{array}{l}\text { MAE } \\
\text { (Min.) }\end{array}$} & \multirow[b]{2}{*}{$\begin{array}{c}\text { MAPE } \\
(\%)\end{array}$} & \multirow[b]{2}{*}{$\begin{array}{c}\text { Reliability } \\
\text { (\%) }\end{array}$} & \multirow[b]{2}{*}{$\begin{array}{c}\% \\
\text { Early }\end{array}$} & \multirow[b]{2}{*}{$\begin{array}{c}\% \\
\text { Late }\end{array}$} \\
\hline & $\begin{array}{c}\text { Systematic } \\
\text { High/Low } \\
\text { Speed }\end{array}$ & $\begin{array}{c}\text { Stations w/ } \\
\text { Errors }\end{array}$ & $\begin{array}{c}\text { Lanes at } \\
\text { Stations w/ } \\
\text { Errors }\end{array}$ & & & & & \\
\hline \multirow{13}{*}{$\begin{array}{l}\text { w/o Data } \\
\text { Filtering }\end{array}$} & \multicolumn{3}{|c|}{ w/o Errors } & 0.08 & 1.31 & 100 & 0 & 0 \\
\hline & \multirow{6}{*}{$\begin{array}{l}\text { Systematic } \\
\text { High Speed }\end{array}$} & 1 Station & All Lanes & 0.12 & 1.91 & 100 & 0 & 0 \\
\hline & & 2 Stations & All Lanes & 0.15 & 2.30 & 100 & 0 & 0 \\
\hline & & 3 Stations & All Lanes & 0.21 & 3.24 & 100 & 0 & 0 \\
\hline & & 4 Stations & 1 Lane & 0.18 & 2.88 & 100 & 0 & 0 \\
\hline & & 4 Stations & 2 Lanes & 0.28 & 4.51 & 100 & 0 & 0 \\
\hline & & 4 Stations & All Lanes & 0.37 & 5.85 & 100 & 0 & 0 \\
\hline & \multirow{6}{*}{$\begin{array}{l}\text { Systematic } \\
\text { Low Speed }\end{array}$} & 1 Station & All Lanes & 0.21 & 3.40 & 86.59 & 13.41 & 0 \\
\hline & & 2 Stations & All Lanes & 0.27 & 4.30 & 86.37 & 13.63 & 0 \\
\hline & & 3 Stations & All Lanes & 0.38 & 5.96 & 87.06 & 12.95 & 0 \\
\hline & & 4 Stations & 1 Lane & 0.10 & 1.67 & 99.34 & 0.66 & 0 \\
\hline & & 4 Stations & 2 Lanes & 0.32 & 5.13 & 90.77 & 9.23 & 0 \\
\hline & & 4 Stations & All Lanes & 0.71 & 11.29 & 83.58 & 16.42 & 0 \\
\hline \multirow{13}{*}{$\begin{array}{l}\text { W/ Data } \\
\text { Filtering }\end{array}$} & \multicolumn{3}{|c|}{ w/o Errors } & 0.08 & 1.31 & 100 & 0 & 0 \\
\hline & \multirow{6}{*}{$\begin{array}{l}\text { Systematic } \\
\text { High Speed }\end{array}$} & 1 Station & All Lanes & 0.11 & 1.72 & 100 & 0 & 0 \\
\hline & & 2 Stations & All Lanes & 0.11 & 1.77 & 100 & 0 & 0 \\
\hline & & 3 Stations & All Lanes & 0.17 & 2.68 & 100 & 0 & 0 \\
\hline & & 4 Stations & 1 Lane & 0.11 & 1.80 & 100 & 0 & 0 \\
\hline & & 4 Stations & 2 Lanes & 0.17 & 2.69 & 100 & 0 & 0 \\
\hline & & 4 Stations & All Lanes & 0.33 & 5.15 & 100 & 0 & 0 \\
\hline & \multirow{6}{*}{$\begin{array}{l}\text { Systematic } \\
\text { Low Speed }\end{array}$} & 1 Station & All Lanes & 0.21 & 3.40 & 86.59 & 13.41 & 0 \\
\hline & & 2 Stations & All Lanes & 0.27 & 4.30 & 86.37 & 13.63 & 0 \\
\hline & & 3 Stations & All Lanes & 0.38 & 5.95 & 87.06 & 12.95 & 0 \\
\hline & & 4 4 Stations & 1 Lane & 0.10 & 1.67 & 99.34 & 0.66 & 0 \\
\hline & & \begin{tabular}{|l}
4 Stations \\
\end{tabular} & 2 Lanes & 0.32 & 5.13 & 90.77 & 9.23 & 0 \\
\hline & & 4 Stations & All Lanes & 0.71 & 11.29 & 83.58 & \begin{tabular}{|l|}
16.42 \\
\end{tabular} & 0 \\
\hline
\end{tabular}


Table 5-5b Impacts of Systematic Errors on Travel Time Estimation Performance for Simulated Incident Conditions

\begin{tabular}{|c|c|c|c|c|c|c|c|c|}
\hline \multirow[b]{2}{*}{$\begin{array}{l}\text { Hybrid } \\
\text { Model } 2\end{array}$} & \multicolumn{3}{|c|}{ Cases } & \multirow[b]{2}{*}{$\begin{array}{l}\text { MAE } \\
\text { (Min.) }\end{array}$} & \multirow[b]{2}{*}{$\begin{array}{c}\text { MAPE } \\
(\%)\end{array}$} & \multirow[b]{2}{*}{$\begin{array}{c}\text { Reliability } \\
\text { (\%) }\end{array}$} & \multirow[b]{2}{*}{$\begin{array}{c}\% \\
\text { Early }\end{array}$} & \multirow[b]{2}{*}{$\begin{array}{c}\% \\
\text { Late }\end{array}$} \\
\hline & $\begin{array}{c}\text { Systematic } \\
\text { High/Low } \\
\text { Speed }\end{array}$ & $\begin{array}{c}\text { Stations w/ } \\
\text { Errors }\end{array}$ & \begin{tabular}{|c|} 
Lanes at \\
Stations w/ \\
Errors
\end{tabular} & & & & & \\
\hline \multirow{13}{*}{$\begin{array}{l}\text { w/o Data } \\
\text { Filtering }\end{array}$} & \multicolumn{3}{|c|}{ w/o Errors } & 1.15 & 10.12 & 74.50 & 3.33 & 22.17 \\
\hline & \multirow{6}{*}{$\begin{array}{l}\text { Systematic } \\
\text { High Speed }\end{array}$} & 1 Station & All Lanes & 1.16 & 10.17 & 74.38 & 2.99 & 22.63 \\
\hline & & 2 Stations & All Lanes & 1.16 & 10.21 & 74.84 & 2.53 & 22.63 \\
\hline & & 3 Stations & All Lanes & 1.25 & 11.05 & 74.38 & 1.49 & 24.12 \\
\hline & & 4 Stations & 1 Lane & 1.99 & 15.88 & 60.02 & 0.23 & 39.75 \\
\hline & & \begin{tabular}{|l|}
4 Stations \\
\end{tabular} & 2 Lanes & 2.37 & 19.00 & 54.62 & 0.23 & 45.15 \\
\hline & & 4 Stations & All Lanes & 2.54 & 20.62 & 53.88 & 0.23 & 45.89 \\
\hline & \multirow{6}{*}{$\begin{array}{l}\text { Systematic } \\
\text { Low Speed }\end{array}$} & 1 Station & All Lanes & 1.50 & 13.11 & 64.39 & 15.11 & 20.51 \\
\hline & & 2 Stations & All Lanes & 1.25 & 10.95 & 71.22 & 7.64 & 21.14 \\
\hline & & \begin{tabular}{|l|}
3 Stations \\
\end{tabular} & All Lanes & 1.10 & 10.47 & 75.07 & 8.73 & 16.20 \\
\hline & & 4 Stations & 1 Lane & 1.58 & 13.26 & 61.86 & 15.91 & 22.23 \\
\hline & & \begin{tabular}{|l|} 
4 Stations \\
\end{tabular} & 2 Lanes & 1.69 & 14.70 & 52.38 & 22.92 & 24.70 \\
\hline & & 4 Stations & All Lanes & 5.62 & 42.88 & 41.53 & 39.06 & 19.41 \\
\hline \multirow{13}{*}{$\begin{array}{l}\text { w/ Data } \\
\text { Filtering }\end{array}$} & \multicolumn{3}{|c|}{ w/o Errors } & 1.15 & 10.12 & 74.50 & 3.33 & 22.17 \\
\hline & \multirow{6}{*}{$\begin{array}{l}\text { Systematic } \\
\text { High Speed }\end{array}$} & 1 Station & All Lanes & 1.15 & 10.09 & 74.38 & 2.99 & 22.63 \\
\hline & & 2 Stations & All Lanes & 1.15 & 10.09 & 74.38 & 2.99 & 22.63 \\
\hline & & 3 Stations & All Lanes & 1.22 & 10.71 & 74.84 & 1.61 & 23.55 \\
\hline & & \begin{tabular}{|l|l}
4 Stations \\
\end{tabular} & 1 Lane & 1.28 & 11.17 & 70.76 & 5.28 & 23.95 \\
\hline & & 4 Stations & 2 Lanes & 1.53 & 13.05 & 67.43 & 2.35 & 30.21 \\
\hline & & 4 Stations & All Lanes & 2.50 & 20.13 & 53.88 & 0.23 & 45.89 \\
\hline & \multirow{6}{*}{$\begin{array}{l}\text { Systematic } \\
\text { Low Speed }\end{array}$} & 1 Station & All Lanes & 1.50 & 13.13 & 64.45 & 15.28 & 20.28 \\
\hline & & 2 Stations & All Lanes & 1.25 & 10.93 & 71.22 & 7.64 & 21.14 \\
\hline & & 3 Stations & All Lanes & 1.08 & 9.78 & 75.53 & 4.65 & 19.82 \\
\hline & & 4 Stations & 1 Lane & 1.22 & 10.78 & 70.42 & 9.02 & 20.56 \\
\hline & & 4 Stations & 2 Lanes & 1.50 & 13.05 & 64.96 & 12.23 & 22.80 \\
\hline & & 4 Stations & All Lanes & 3.46 & 25.72 & 55.43 & 15.16 & 29.41 \\
\hline
\end{tabular}

The accuracy of some types of point detectors decrease under low speed conditions. To test how such errors in low speed measurements affect the accuracy and reliability of travel time estimation methods, systematic errors are introduced to the error-free simulated detector data when speed is less than $20 \mathrm{mph}$, and the analysis results 
are presented below by using Hybrid Model 2 as an example. It is seen from Table 5-6 that when the measured low speeds are artificially increased or decreased by $20 \%$, the error of the estimated travel time increass slightly from $10.1 \%$ to about $12 \%$. However, with an introduced systematic error of $40 \%$ in the measured speeds, the estimated error in travel time increased by $4 \%$ to $5 \%$, compared to the case without errors in measured speeds. The reliability of travel time estimates decreases significantly when the point detector systematically reports lower speeds than the actual values during the congested conditions, as shown in Table 5-6. Note that the results presented in Table 5-6 are obtained with data filtering procedures.

Table 5-6 Impacts of Systematic Errors in Low Speed Measurements on Travel Time Estimation Performance for Simulated Incident Conditions

\begin{tabular}{|l|c|c|c|c|c|c|}
\hline \multirow{2}{*}{ Method } & Cases & $\begin{array}{c}\text { MAE } \\
\text { (Min.) }\end{array}$ & $\begin{array}{c}\text { MAPE } \\
(\mathbf{\%})\end{array}$ & $\begin{array}{c}\text { Reliability } \\
(\mathbf{\%})\end{array}$ & $\begin{array}{c}\text { \% } \\
\text { Early }\end{array}$ & $\begin{array}{c}\text { \% } \\
\text { Late }\end{array}$ \\
\hline \multirow{4}{*}{ Hybrid Model 2 } & w/o Errors & 1.15 & 10.12 & 74.50 & 3.33 & 22.17 \\
\cline { 2 - 7 } & 20\% Increase in Low Speed & 1.37 & 11.52 & 71.97 & 1.21 & 26.82 \\
\cline { 2 - 7 } & 40\% Increase in Low Speed & 1.66 & 13.57 & 68.18 & 0.98 & 30.84 \\
\cline { 2 - 7 } & 20\% Decrease in Low Speed & 1.46 & 12.33 & 61.69 & 16.94 & 21.37 \\
\cline { 2 - 7 } & 40\% Decrease in Low Speed & 1.88 & 15.19 & 57.15 & 16.83 & 26.02 \\
\hline
\end{tabular}

\subsubsection{Incidental and Structural Failure}

In addition to the two types of errors mentioned above, incidental and/or structural failures may also exist in detector data (Vant Lint 2004). The incidental or occasional failure occurs randomly. Various factors may contribute to its occurrence, such as temporary communication system failure resulting from power outrages. Figure 5-3 presents histograms of incidental and structural failures for detectors DS-1509E-Lane-1 and DS-1549E-Lane-1, as examples, and also for all the detectors along SR-826 in December, 2008. Note that the units of the x-axis in Figures 5-3(a) and 5-3(c) are 
minutes and those for Figures 5-3(b) and 5-3(d) are hours. Most of the missing data duration is less than 2 minutes.

To quantify the impacts of incidental and structural failures, worst case scenarios were created by introducing failures to the error-free simulated detector data. These failure scenarios were created based on what happens in real-world detector data during the study period in December, 2008. Since a detector may fail more than once, the longest duration of the missing data during the failure period was used.

Table 5-7 shows the impacts of incidental and structural failures on travel time estimation performance during both uncongested and incident conditions. The results in this table show that, even with the worst case of incidental and structural failures, after imputing the missing data, these failures do not have high impacts on the estimated travel time accuracy for the uncongested conditions. However, for the incident scenario, the existence of incidental and structural failures can result in a large increase in estimation errors and also a reduction in reliability. This can be explained by the fact that even though the data imputation procedure is applied to replace the missing data during incident conditions, due to fast changes in traffic conditions, the filled data may not be able to completely capture such changes, resulting in a less satisfactory performance. 


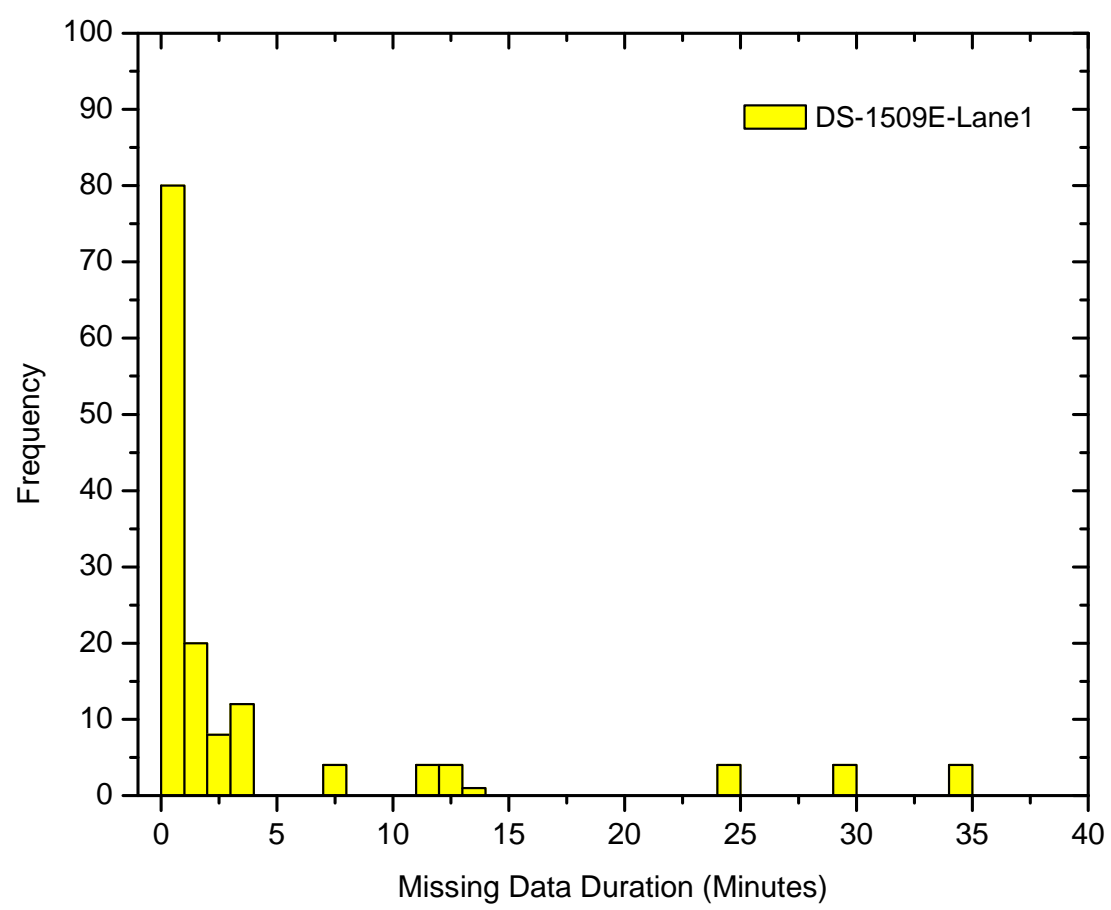

(a)

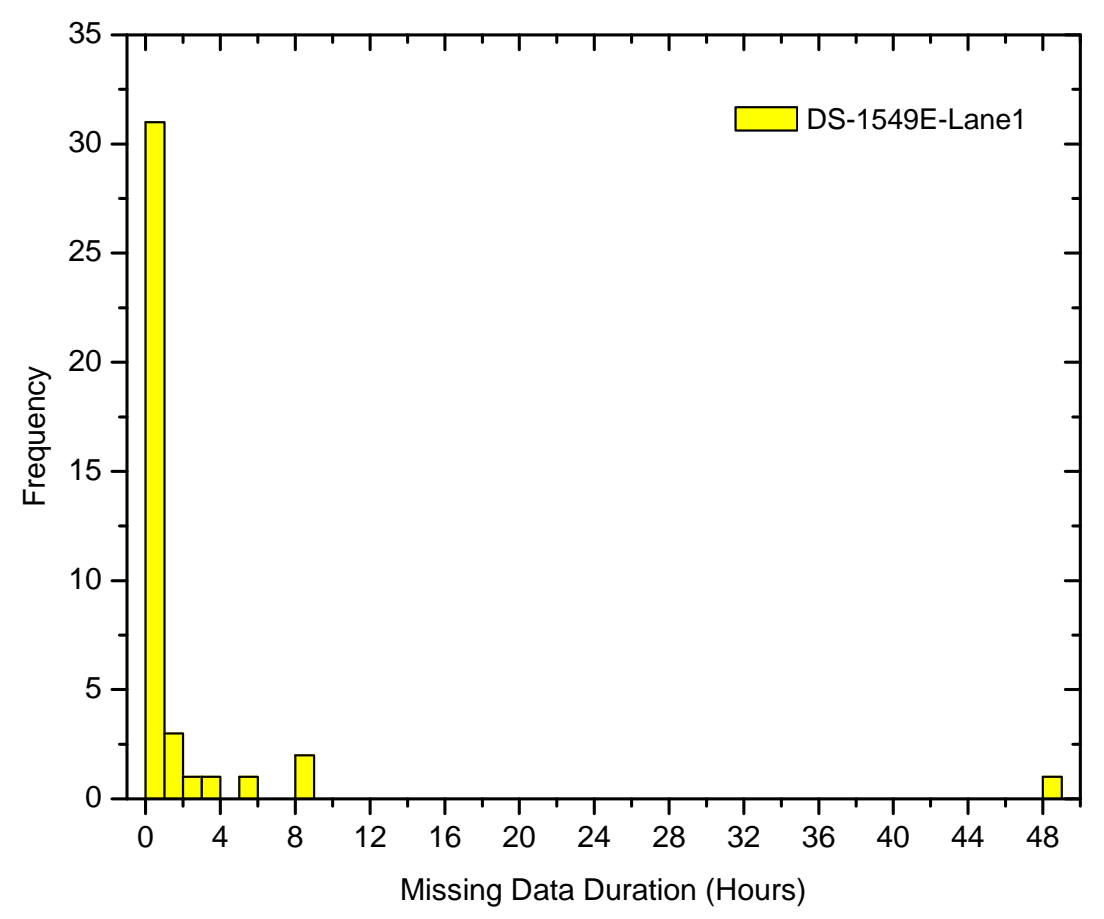

(b)

Figure 5-3 Examples of Incidental and Structural Failures (continued on next page) 


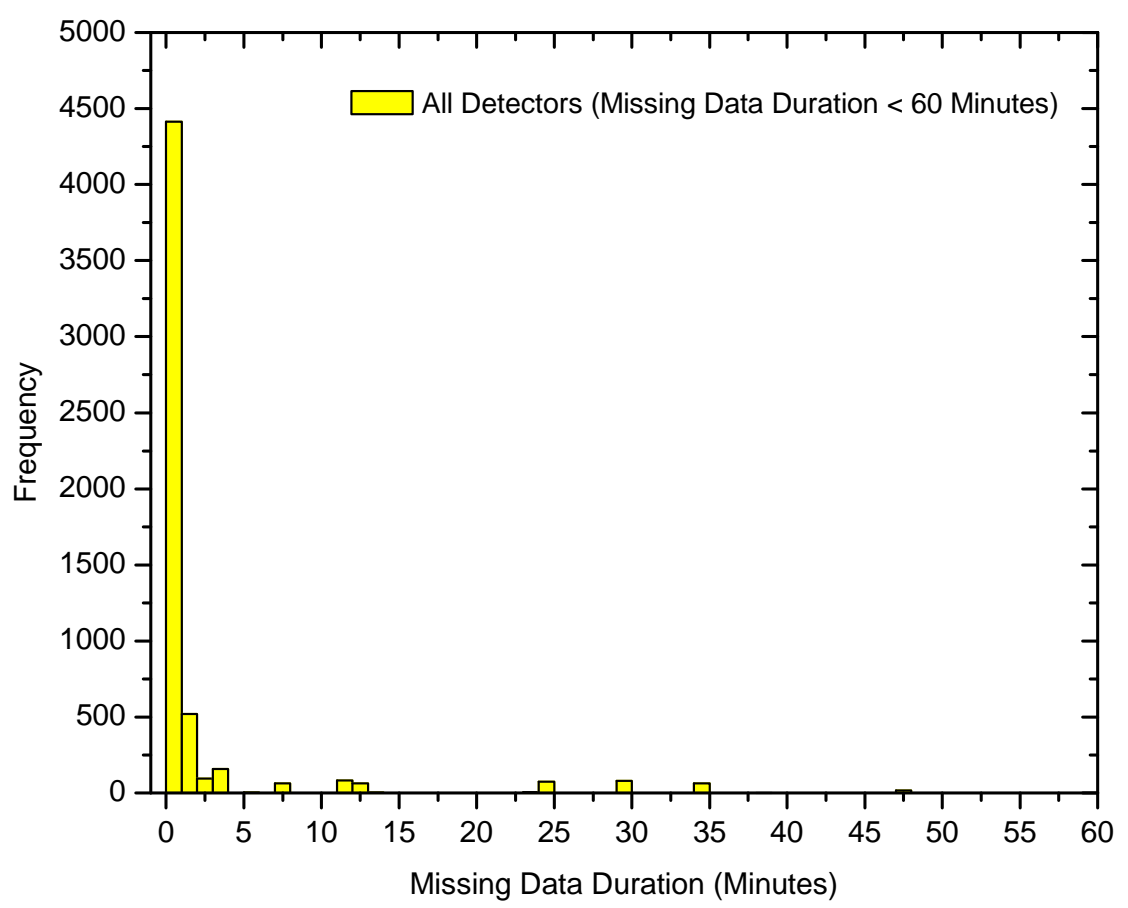

(c)

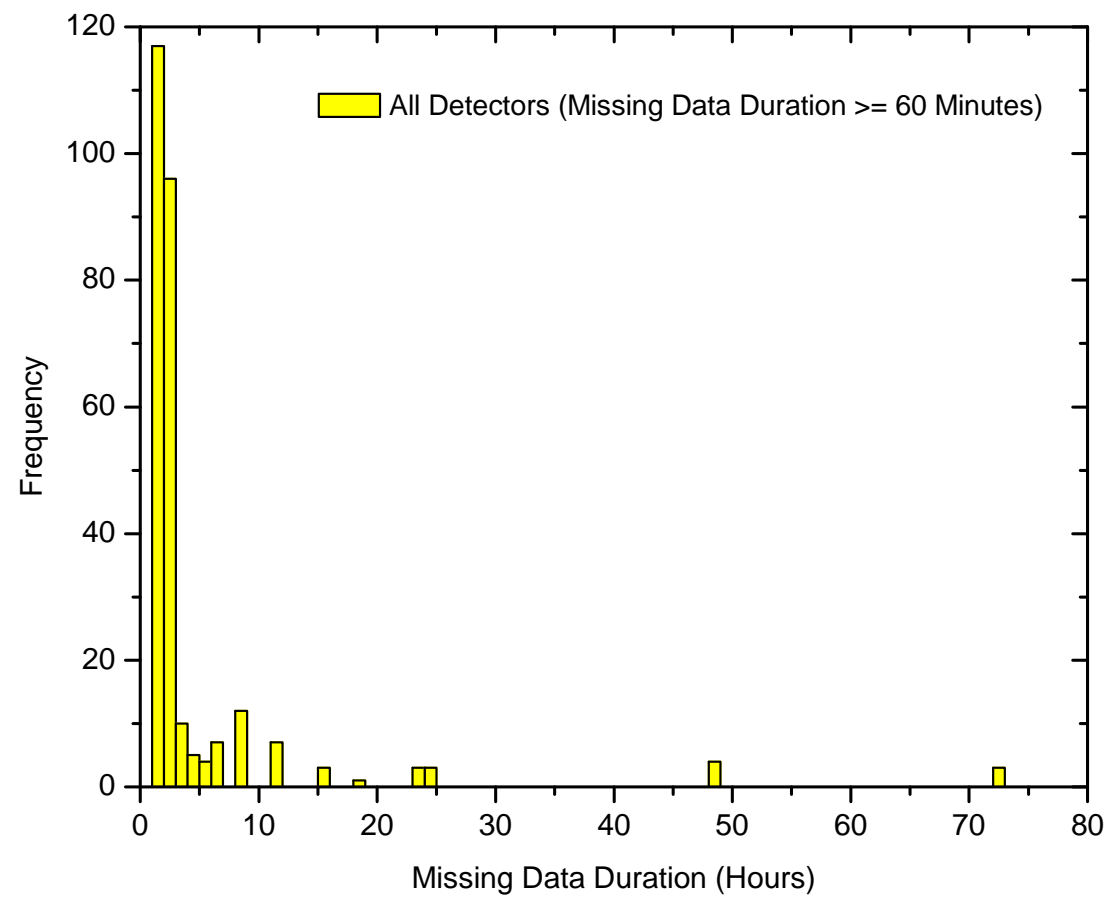

(d)

Figure 5-3 Examples of Incidental and Structural Failures 
Table 5-7 Impacts of Incidental and Structural Failures on Travel Time Estimation Performance

\begin{tabular}{|c|c|c|c|c|c|c|}
\hline Hybrid Model 2 & Cases & $\begin{array}{l}\text { MAE } \\
\text { (Min.) } \\
\end{array}$ & $\begin{array}{c}\text { MAPE } \\
(\%) \\
\end{array}$ & \begin{tabular}{|c|}
$\begin{array}{c}\text { Reliability } \\
(\%)\end{array}$ \\
\end{tabular} & \% Early & \% Late \\
\hline \multirow{2}{*}{$\begin{array}{l}\text { Simulated } \\
\text { Uncongested } \\
\text { Conditions }\end{array}$} & w/o Errors & 0.08 & 1.31 & 100 & 0 & 0 \\
\hline & $\begin{array}{l}\text { w/ Incidental and } \\
\text { Structural Errors }\end{array}$ & 0.10 & 1.54 & 100 & 0 & 0 \\
\hline \multirow{2}{*}{$\begin{array}{l}\text { Simulated } \\
\text { Incident } \\
\text { Conditions }\end{array}$} & w/o Errors & 1.15 & 10.12 & 74.50 & 3.33 & 22.17 \\
\hline & $\begin{array}{l}\text { w/ Incidental and } \\
\text { Structural Errors }\end{array}$ & 1.95 & 17.58 & 62.44 & 15.45 & 22.11 \\
\hline
\end{tabular}

\subsection{Detector Spacing}

Number of detectors as well as their locations may affect the travel time estimation performance. In order to study such impacts, some simulated detector data are intentionally removed to increase the average detector spacing. Table 5-8 presents the sensitivity analysis results for the impacts of detector spacing. As mentioned in the previous chapter, the average spacing between detectors deployed along the eastbound of SR-826 is about 0.3 miles. As shown in Table 5-8, for uncongested traffic conditions, an increase in detector spacing only slightly reduces the accuracy and reliability of travel time estimation, as the traffic is relatively stable in this case. However, the performance of travel time estimation is greatly affected with the increase in detector spacing for incident scenario. For example, as the detector spacing increases from about 0.3 miles to 0.6 miles, the MAPE increases from $10.1 \%$ to $19.7 \%$, and the reliability decreases from $74.5 \%$ to $52.8 \%$. This is because large detector spacing cannot capture the changes in traffic dynamics. With the further increase in detector spacing from 0.6 miles to about 1.2 miles, the performance of travel time estimation appears to be almost the same for investigated conditions. 
Table 5-8 Impacts of Detector Spacing on Travel Time Estimation Performance

\begin{tabular}{|l|c|c|c|c|c|c|}
\hline Hybrid Model 2 & $\begin{array}{c}\text { Approximate } \\
\text { Detector Spacing } \\
\text { (Miles) }\end{array}$ & $\begin{array}{c}\text { MAE } \\
\text { (Min.) }\end{array}$ & $\begin{array}{c}\text { MAPE } \\
\mathbf{( \% )}\end{array}$ & $\begin{array}{c}\text { Reliability } \\
\mathbf{( \% )}\end{array}$ & \% Early & \% Late \\
\hline $\begin{array}{l}\text { Simulated } \\
\text { Uncongested } \\
\text { Conditions }\end{array}$ & 0.3 & 0.08 & 1.31 & 100 & 0 & 0 \\
\cline { 2 - 7 } & 0.6 & 0.10 & 1.57 & 100 & 0 & 0 \\
\hline \multirow{2}{*}{$\begin{array}{l}\text { Simulated } \\
\text { Incident } \\
\text { Conditions }\end{array}$} & 1.2 & 0.09 & 1.40 & 99.75 & 0.25 & 0 \\
\cline { 2 - 7 } & 0.3 & 1.15 & 10.12 & 74.50 & 3.33 & 22.17 \\
\cline { 2 - 7 } & 0.6 & 2.56 & 19.74 & 52.84 & 1.15 & 46.01 \\
\hline
\end{tabular}

\subsection{Travel Time Posting Configuration}

Travel time posting strategies, such as travel time updating frequency, travel time link length, and the range of posted travel time are also expected to affect the accuracy and reliability of travel time estimates. This section includes the results of tests conducted in this study to investigate the impacts of these influential factors.

\subsubsection{Travel Time Updating Frequency}

Sensitivity analysis is conducted in this study to understand the impacts of the travel time updating frequency on the accuracy and reliability of travel time estimates. Updating frequencies ranging from one to five minutes are included in the analysis. Table 5-9 displays the travel time estimation results for the simulated uncongested conditions and incident conditions as well. The results in this table indicate that for the uncongested conditions, a longer travel time updating interval does not lead to worse estimation performance, since the traffic is relatively stable under uncongested conditions. But for the incident scenario, it is seen that the errors increase and that the reliability is reduced with an increase in the travel time updating interval. This indicates that more frequent 
updates in travel time estimates are preferred for incident conditions due to the varying traffic conditions during incidents.

Table 5-9 Travel Time Estimation Performances with Different Travel Time Updating Frequencies

\begin{tabular}{|l|c|c|c|c|c|c|}
\hline Hybrid Model 2 & $\begin{array}{c}\text { Updating } \\
\text { Frequency }\end{array}$ & $\begin{array}{c}\text { MAE } \\
\text { (Min.) }\end{array}$ & $\begin{array}{c}\text { MAPE } \\
\text { (\%) }\end{array}$ & $\begin{array}{c}\text { Reliability } \\
\mathbf{( \% )}\end{array}$ & \% Early & \% Late \\
\hline \multirow{4}{*}{$\begin{array}{l}\text { Simulated } \\
\text { Cncongested }\end{array}$} & 1-minute & 0.10 & 1.62 & 100 & 0 & 0 \\
\cline { 2 - 7 } & 2-minute & 0.08 & 1.31 & 100 & 0 & 0 \\
\cline { 2 - 7 } & 3-minute & 0.07 & 1.16 & 100 & 0 & 0 \\
\cline { 2 - 7 } & 4-minute & 0.07 & 1.15 & 100 & 0 & 0 \\
\cline { 2 - 7 } & 5-minute & 0.07 & 1.06 & 99.89 & 0.11 & 0 \\
\hline \multirow{4}{*}{$\begin{array}{l}\text { Simulated } \\
\text { Incident }\end{array}$} & 1-minute & 1.16 & 10.21 & 74.24 & 2.58 & 23.18 \\
\cline { 2 - 7 } & 2-minute & 1.15 & 10.12 & 74.50 & 3.33 & 22.17 \\
\cline { 2 - 7 } & 3-minute & 1.31 & 11.31 & 70.55 & 4.64 & 24.81 \\
\cline { 2 - 7 } & 4-minute & 1.35 & 11.85 & 55.38 & 17.69 & 26.93 \\
\cline { 2 - 7 } & 5-minute & 1.34 & 11.25 & 69.56 & 6.86 & 23.58 \\
\hline
\end{tabular}

\subsubsection{Travel Time Link Length}

To show the impacts of travel time link lengths, four different travel time links are defined as follows:

- DS-1523E - DS-1549E (Distance: 4.24 miles)

- DS-1521E - DS-1549E (Distance: 4.55 miles)

- DS-1517E - DS-1549E (Distance: 5.27 miles)

- DS-1509E - DS-1549E (Distance: 6.42 miles)

Table 5-10 presents the travel time estimation accuracy and reliability for these defined travel time links under the simulated uncongested and Incident 1 scenarios. The mean absolute errors are shown to increase with the increase in the link length. However, the mean absolute percentage errors do not monotonically change with such an increase in distance as this performance measure is also related to the actual travel time 
spatial distribution along the studied travel time link. The reliability does not necessarily decreases as the distance increases since the reliability is also determined by the range of posted travel time. Similar conclusions can be obtained based on the results in Table 5-10 for incident conditions. Any conclusions based on the reported results are limited to the range of the increase in length investigated in this study.

Table 5-10 Travel Time Estimation Performances with Different Travel Time Link Lengths

\begin{tabular}{|c|c|c|c|c|c|c|c|}
\hline $\begin{array}{l}\text { Hybrid } \\
\text { Model } 2 \\
\end{array}$ & Origin-Destination & $\begin{array}{c}\text { Distance } \\
\text { (Miles) }\end{array}$ & $\begin{array}{l}\text { MAE } \\
\text { (Min.) }\end{array}$ & $\begin{array}{c}\text { MAPE } \\
(\%)\end{array}$ & $\begin{array}{c}\text { Reliability } \\
(\%)\end{array}$ & $\begin{array}{c}\% \\
\text { Early } \\
\end{array}$ & $\begin{array}{c}\% \\
\text { Late } \\
\end{array}$ \\
\hline \multirow{4}{*}{$\begin{array}{l}\text { Simulated } \\
\text { Uncongested } \\
\text { Conditions }\end{array}$} & & \begin{tabular}{|l|}
4.24 \\
\end{tabular} & 0.05 & 1.16 & 100 & 0 & 0 \\
\hline & DS-1521E-DS-1549E & 4.55 & 0.05 & 1.15 & 99.69 & 0.08 & 0.23 \\
\hline & DS-1517E-DS-1549E & 5.27 & 0.06 & 1.09 & 100 & 0 & 0 \\
\hline & DS-1509E-DS-1549E & 6.42 & 0.08 & 1.31 & 100 & 0 & 0 \\
\hline \multirow{4}{*}{$\begin{array}{l}\text { Simulated } \\
\text { Incident } \\
\text { Conditions }\end{array}$} & DS-1523E-DS-1549E & 4.24 & 0.88 & 13.69 & 91.40 & 0 & 8.60 \\
\hline & DS-1521E-DS-1549E & 4.55 & 1.05 & 13.50 & 81.94 & 4.59 & 13.47 \\
\hline & DS-1517E-DS-1549E & 5.27 & 1.05 & 10.90 & 75.52 & 3.38 & 21.10 \\
\hline & DS-1509E-DS-1549E & 6.42 & 1.15 & 10.12 & 74.50 & 3.33 & 22.17 \\
\hline
\end{tabular}

\subsubsection{Posted Travel Time Range}

As mentioned above, FDOT District 6 divides the estimated travel time into four categories: less than 5 minutes, between 5 and 10 minutes, between 10 and 35 minutes, and greater than 35 minutes (FDOT District 6 2010). The corresponding ranges of travel time for these four categories are under 5 minutes, 3-minute range around the estimated travel time, 5-minute range around the estimated travel time, and over 35 minutes, respectively.

Sensitivity analysis is conducted to see how the reliability of travel time estimation changes by adjusting the posted travel time range. The corresponding estimation performance is listed in Table 5-11. This table shows that for uncongested 
conditions, the reliability of the posted travel time is close to $100 \%$ even when the posted travel time range is decreased to 2 minutes. However, if the travel time range is further reduced to 1 minute, the reliability of the estimated travel time is significantly impacted.

Table 5-11 Travel Time Estimation Reliability with Different Posted Travel Time Ranges

\begin{tabular}{|l|l|c|c|c|}
\hline Hybrid Model 2 & $\begin{array}{l}\text { Range of Posted } \\
\text { Travel Time }\end{array}$ & $\begin{array}{c}\text { Reliability } \\
\mathbf{( \% )}\end{array}$ & \% Early & \% Late \\
\hline \multirow{3}{*}{$\begin{array}{l}\text { Simulated Uncongested } \\
\text { Conditions }\end{array}$} & {$[\mathrm{TT}-2, \mathrm{TT}+2]$} & 100 & 0 & 0 \\
\cline { 2 - 5 } & {$[\mathrm{TT}-1, \mathrm{TT}+2]$} & 100 & 0 & 0 \\
\cline { 2 - 5 } & {$[\mathrm{TT}-1, \mathrm{TT}+1]$} & 99.29 & 0 & 0.71 \\
\cline { 2 - 5 } & {$[\mathrm{TT}-0.5, \mathrm{TT}+0.5]$} & 70.07 & 0.42 & 29.52 \\
\hline \multirow{4}{*}{$\begin{array}{l}\text { Simulated Incident } \\
\text { Conditions }\end{array}$} & {$[\mathrm{TT}-2, \mathrm{TT}+3]$} & 74.50 & 3.33 & 22.17 \\
\cline { 2 - 5 } & {$[\mathrm{TT}-2, \mathrm{TT}+4]$} & 75.88 & 3.33 & 20.79 \\
\cline { 2 - 5 } & {$[\mathrm{TT}-2, \mathrm{TT}+5]$} & 75.88 & 3.33 & 20.79 \\
\cline { 2 - 5 } & {$[\mathrm{TT}-1, \mathrm{TT}+4]$} & 69.67 & 9.54 & 20.79 \\
\cline { 2 - 5 } & {$[\mathrm{TT}-1, \mathrm{TT}+5]$} & 69.67 & 9.54 & 20.79 \\
\cline { 2 - 5 } & {$[\mathrm{TT}-1, \mathrm{TT}+6]$} & 70.25 & 9.54 & 20.22 \\
\hline
\end{tabular}

Note that the posted travel time range in Table 5-11 for incident conditions is mainly for travel time estimates that are greater than 10 minutes, due to the congestion caused by the incident. It can be seen from this table that if the upper value of travel time range is increased to higher than 3 minutes, the reliability of the estimated travel time does not improve much. This indicates that such an adjustment is not beneficial. Furthermore, decreasing the lower range to 1 minute reduces overall reliability.

\subsection{Summary}

The impacts of data preprocessing procedures, detector errors, detector spacing, and travel time posting strategies on travel time estimation accuracy and reliability were investigated through sensitivity analysis. Two data smoothing methods were compared: the simple moving average method and exponential moving average method. The 
results show that the exponential moving average method can produce better results during incident conditions as this method gives more weights to the latest data. The comparison of different data imputation methods indicated that the imputation method used in the SunGuide software appears to perform as good as other investigated methods.

Three types of detector errors, intrinsic errors, systematic errors, and incidental and structural failures, were examined by intentionally introducing errors to $100 \%$ accurate simulated detector data. The performances of travel time estimation based on these detector data with errors were compared to those estimated from $100 \%$ accurate detector data. The analysis results show that detector errors have negligible impacts on travel time estimates during the uncongested conditions within the investigated ranges of errors; however, the performance of travel time estimation can deteriorate significantly in incident scenarios. Similarly, the impacts of detector spacing were examined by removing certain detector data. The sensitivity analysis results indicate that congested conditions such as incidents require closer detector spacing.

Travel time posting configurations were considered in this study in terms of travel time updating frequency, travel time link length, and posted travel time range. It is found that for uncongested conditions, travel time can be estimated accurately and reliably even with a longer updating interval, longer travel time link length, and small range of posted travel time. However, for incident conditions, the travel time estimation requires a shorter updating frequency (such as 1-minute), shorter travel time link length, and a wider posting range to achieve a better performance. 


\section{CHAPTER 6}

\section{RESEARCH SUMMARY}

This study has developed two hybrid on-line travel time estimation models and two corresponding off-line methods to estimate freeway travel times based on point detector measurements. The accuracy and reliability of travel time estimation using these models were compared to the existing speed-based and traffic flow-based methods using simulation and real-world data. In addition, the performance of travel time estimation under different conditions, such as different congestion levels, incident conditions, detector errors, and various estimation method basic parameters, was also investigated. This chapter summarizes the original contributions of the proposed methodology and conclusions of the study, and discusses the direction of future work.

\subsection{Study Contribution}

Compared to existing travel time estimation methods, the main contributions of this study are the following:

- A clustering analysis-based traffic condition identification procedure was incorporated in travel time estimation. This provides an automatic way to identify congestion level and queue status, and in turn provides a threshold for switching between different travel time estimation methods, as described next.

- Based on the performance of individual estimation methods under certain conditions, two hybrid models were developed. Hybrid Model 1 combines the Mid-Point method with a traffic flow-based method. Hybrid Model 2 combines the Mid-Point method with the Minimum Speed method. Such 
combinations may achieve better performance since different methods perform differently under different traffic conditions, including no-congestion, recurrent conditions and incidents.

- In addition, instead of assuming one value for the average effective vehicle length used in the traffic flow-based estimation method, an effective way to compute this parameter based on detector data in the same peak period was proposed in this study.

- During incident conditions with fast changing queue lengths, shock wave analysis-based refinements were introduced to the developed models to account for the fast queue prorogation and recovery.

- Both on-line and off-line hybrid models were developed and compared in this study; this provides some insight into the conditions under which the accuracy and reliability of travel time estimates can potentially be improved.

- In addition to traditional accuracy measures, performance measures, in terms of percentage of vehicles arriving to the destination within a given travel time range, percentage of vehicles arriving early, and percentage of vehicles arriving late, were proposed in this study to quantify the reliability of travel time estimation. This is closely related to the practice of posting travel time as a range of travel time instead of an exact value, which is usually posted in real-world applications.

- Detector errors, including intrinsic errors, systematic errors, and incidental and structural failures, were introduced to the error-free simulated detector 
data to study how detector errors affect the travel time estimation accuracy and reliability.

\subsection{Conclusions}

In this study, travel time estimates obtained from existing speed-based methods, traffic flow-based method, and the developed models were tested by using both simulation and real-world travel time data as ground truth data. The results indicate that all of the tested methods perform at acceptable and comparable levels at low congestion levels. However, their performances vary with the increase in congestion levels. During low congestion levels, the Minimum Speed method and flow-based methods produce slightly less accurate results compared to other methods. But the difference is not significant. For moderately recurrent congested conditions and fast-changing conditions during incidents, the performances of these two methods are comparable to the performances of other methods. The comparison with other estimation methods shows that the developed hybrid models perform well in all cases. Further comparisons between the on-line and off-line travel time estimation results reveal that off-line methods perform significantly better only during fast-changing congested conditions such as during incidents. The difference in performance between the on-line and off-line methods increases with the increase in congestion levels.

The impacts of major influential factors, such as data preprocessing procedures, detector errors, detector spacing, and travel time posting strategies, on the performance of travel time estimation, were also investigated in this study. The results indicate that the spatial imputation method used in the SunGuide software to account for missing data 
appears to perform as good as other investigated methods. When estimating travel time during incident conditions, the use of the exponential moving average produces more accurate and reliable results compared to the simple moving average method used in SunGuide, since the exponential moving average method can give more weight to the latest data in the smoothing and can thus better account for the fast-changing dynamic conditions during incidents. When using the simple moving average method during incident conditions, shorter rolling time periods produce better results.

The results also show that the intrinsic errors caused by measurement noise, systematic errors (e.g., due to inadequate calibration or device inaccuracy), and data missing due to incidental and/or structural failure have significantly higher negative impacts on the performance of travel time estimation during congested conditions than for uncongested conditions. Similarly, larger detector spacing does not worsen the performance of travel time estimation during uncongested conditions, but has significant impacts on the estimation accuracy and reliability during incidents.

The results of the sensitivity analysis in this study indicates that for uncongested conditions, a longer travel time updating interval does not lead to poorer estimation performance. For incident scenarios, the errors increase and the reliability decreases with the increase in the travel time updating interval. The absolute errors also increase with the increase in the travel time link length under incident conditions. With regard to the range of travel time posted on ATIS devices, it appears that a posted travel time range of two-to-three minutes generally produces good results for uncongested conditions. However, if the travel time range is further reduced to one minute, the reliability of the estimated travel time is significantly impacted. For incident scenarios, a slightly larger 
travel time range may increase the reliability of travel time estimation, but the improvement is not significant.

\subsection{Future Work}

The hybrid models for freeway travel time estimation developed in this study are not perfect, and merit some further studies in the following areas:

- For real-time applications, a refinement needs to be made to the developed models at the later stages of lane blockage to capture the effect of front recovery shock wave during incident clearance. However, this requires the knowledge of approximate lane blockage duration. Future efforts may focus on incorporating existing incident duration models including lane blockage duration models in travel time estimation.

- Even though the developed hybrid models were tested using simulated incidents, it is not clear how these models perform when applying them to real-world incident cases. It is necessary to collect real-world travel time data, especially during various types of incidents, to further test these models.

- The off-line hybrid models developed in this study can be used in the training process of real-time short-term travel time prediction approaches such as Neural Network, whose performances may be compared to the developed on-line hybrid models in future studies.

- The reliability of travel time estimation was only quantified based on simulation. Due to lack of data, this study did not quantify the estimation reliability for real-world cases. In the future, additional real-world data, such as vehicle 
trajectory data, or travel time distribution data, should be collected and used in the evaluation of travel time estimation reliability.

- There is limited information regarding point detector accuracy under various conditions. Experiments should be conducted to test the measurement accuracy of different types of detectors, especially during low speed conditions. This information can be further used in the methodology developed by this study to investigate their impacts on travel time estimation performance. 


\section{REFERENCES}

AASHTO (1992). “AASHTO Guidelines for Traffic Data Programs.” American Association of State Highway and Transportation Officials, Washington, D.C. pp. 114.

Abdulhai, B., Porwal, H., and Recker, W. (1999). "Short Term Freeway Traffic Flow Prediction Using Genetically-Optimized Time-Delay-Based Neural Networks.” Presented at 78th Annual Meeting of Transportation Research Board, Washington, D.C.

Al-Deek, H., Kerr, P., Ramachandran, B., Chehab, A., Pooley, J., Emam, E., Chandra, R., Petty, K., and Swinson, I. (2004). "The Central Florida Data Warehouse (CFDW) Phase-2, The Central ITS Office Funding.” Final Report.

Ametha, J., Turner, S., and Darbha, S. (2001). "Formulation of a New Methodology to Identify Erroneous Paired Loop Detectors.” Proceedings of the 2001 IEEE Intelligent Transportation Systems Conference, Oakland, California, pp. 591-596.

Ban, X., Chu, L., and Benouar, H. (2007). "Bottleneck Identification and Calibration for Corridor Management Planning." Transportation Research Record: Journal of the Transportation Research Board, No. 1999, pp. 40-53.

Bajwa, S. I., Chung, E., and Kuwahara, M. (2005). "Performance Evaluation of an Adaptive Travel Time Prediction Model.” Proceedings of the 8th International IEEE Conference on Intelligent Transportation Systems, Vienna, Austria, pp. 1000-1005.

Ben-Akiva, M., Bierlaire, M., Burton, D., Koutsopoulos, H. N., and Mishalani, R. (2001). "Network State Estimation and Prediction for Real-Time Transportation Management Applications.” Networks and Spatial Economics, Vol. 1, No. 3/4.

Bertini, R. L., and Lovell, D. J. (2008). "Developing an Optimal Sensing Strategy for Accurate Freeway Travel Time Estimation and Traveler Information.” Proceedings of the 87th Annual Meeting of the Transportation Research Board, Washington D.C.

Bogers, E. A. I., Van Lint, J. W. C., and Van Zuylen, H. J. (2008). "Travel Time Reliability: Effective Measures from a Behavioral Point of View." Transportation Research Board 87th Annual Meeting (CD-ROM), National Academies Press, Washington D.C.

Byon, Y., Shalaby, A., Abdulhai, B., and El-Tantawy, S. (2009). "Traffic Data Fusion Using SCAAT Kalman Filters.” In Transportation Research Board 88th Annual Meeting. CD-ROM. National Academies Press, Washington D.C. 
Chan, T. N. (2003). "Real-Time Identifaction and Tracking of Recurrent Traffic Queues Based on Average Link Speed Using Loop Detector Data.” Proceedings of the 2003 Canadian Transportation Research Forum (CTRF) Annual Conference, Ottawa, ON.

Chen, C., Kwon, J., Rice, J. (2003). “A. Skabardonic, and P. Varaiya. Detecting Errors and Imputing Missing Data for Single-Loop Surveillance Systems.” Transportation Research Record: Journal of the Transportation Research Board, No. 1855, pp. 160-167.

Chen, C., Skabardonis, A., Varaiya, P. (2003). "Travel Time Reliability as a Measure of Service." Transportation Research Record: Journal of the Transportation Research Board, No. 1855, pp. 74-79.

Chen, L., and May, A. D. (1987). "Traffic Detector Errors and Diagnostics.” Transportation Research Record: Journal of the Transportation Research Board, No. 1132, pp. 82-93.

Chen, M., and Xia, J., and Liu, R. (2006). "Developing a Strategy for Imputing Missing Traffic Volume Data.” Journal of the Transportation Research Forum, Vol. 45, No. 3, pp. 57-75.

Chien, S. I., Liu, X., and Ozbay, K. (2003). "Predicting Travel Times for the South Jersey Real-Time Motorist Information System.” Transportation Research Board Annual Meeting (CD-ROM), National Academies Press, Washington D.C.

Coifman, B. (1998). "Vehicle Reidentification and Travel Time Measurement in Real-Time on Freeways Using the Existing Loop Detector Infrastructure.” Transportation Research Record: Journal of the Transportation Research Board, No. 1643, pp. 181-191.

Coifman, B., and Cassidy, M. (2002). "Vehicle Reidentification and Travel Time Measurement on Congested Freeways.” Transportation Research: Part A., Vol. 36, No. 10, pp. 899-917.

Coifman, B. and Ergueta, E. (2003). "Improved Vehicle Reidentification and Travel Time Measurement on Congested Freeways.” Journal of Transportation Engineering, ASCE, Vol. 129, No. 5, pp. 475-483.

Coifman, B. (2004). “An Assessment of Loop Detector and RTMS Performance.” California PATH Research Report, UCB-ITS-PRR-2004-30.

Conklin, J. H., and Scherer, W. T. (2003). "Data Imputation Strategies for Transportation Management Systems.” Technical Report, UVACTS-13-0-80.

Courage, K., Showers, R., Harriot, J., Schilling, W., and Godbey. K. (1998). "Improved Methods for Measuring Travel Time on Arterial Streets.” Final Report. FL/DOT-99700-3519-119. 
Dailey, D. J. (1993). "Travel-time Estimation Using Cross-correlation Techniques." Transportation Research, Part B, Vol. 27B, No. 2, pp. 97-107.

DE Moor, B., and Immers, L. H. (2006). "Modelling Traffic on Motorways: State-of-the-Art, Numerical Data Analysis, and Dynamic Traffic Assignment.” Katholieke University Leuven, Heverlee, Belgium.

Denning, N. E. (2007). "Implementation and Validation of a Cost-Effective Networked Radar-Based Highway Traffic Speed Measurement System.” Thesis. The Ohio State University.

Dharia, A., and Adeli, H. (2003). "Neural Network Model for Rapid Forecasting of Freeway Link Travel Time.” Engineering Application of Artificial Intelligence, Vol.16, pp.607-613.

Dhulipala, S. (2002). “A System for Travel Time Estimation on Urban Freeways.” MS Thesis, Virginia Polytechnic Institute and State University, Blacksburg, Virginia.

D’Angelo, M. P., Al-Deek, H. M., and Wang, M. C. (1999). “Travel Time Prediction for Freeway Corridors." Transportation Research Record: Journal of the Transportation Research Board, No. 1676, pp. 184-191.

Dubuisson, M-P, Jain, A. K., and Taylor, W. C. (1993). "Segmentation and Matching of Vehicles in Road Images." Transportation Research Record: Journal of the Transportation Research Board, No. 1412, pp. 57-63.

Edara, P., Guo, J., Smith, B. L., and McGhee, C. (2008) "Optimal Placement of Point Detectors on Virginia's Freeways: Case Studies of Northern Virginia and Richmond.” Final Contract Report, VTRC 08-CR3.

EIS. RTMS by EIS, A Simple Solution to Traffic Counting. http://ntl.bts.gov/lib/10000/10000/10041/EIS1.pdf, Accessed August 31, 2010.

Fernández-Moctezuma R. J., Bertini, R. L., Maier, D., and Tufte, K. A. (2009). “Toward Improved and Transparent Imputation Techniques for Online Traffic Data Streams and Archiving Applications." Proceedings of to the 88th Annual Meeting of the Transportation Research Board, Washington, D.C.

Florida Department of Transportation District 6, How Will Travel Time Messaging Tell Motorists about Travel Times, http://www.sunguide.org/sunguide/index.php?/faq/. Accessed July 26, 2010.

Gaudio, R. (2008). "Effect of Segment Length and Congestion Level on Reliability of Travel Time Estimation.” Project Report, Florida International University. 
Geng, Y., and Wu, X. (2008). "The Erroneous Data Imputation Models for Beijing's Urban Traffic Flow Data by Time Series and Correlation Analysis.” Transportation Research Board 87th Annual Meeting (CD-ROM), National Academies Press, Washington D.C.

Guin, A. (2006). "Travel Time Prediction Using a Seasonal Autoregressive Integrated Moving Average Time Series Model.” Proceedings of the IEEE ITSC 2006, 2006 IEEE Intelligent Transportation Systems Conference, Toronto, Canada, pp. 493-498.

Guo, H. and Jin, J. (2006). "Travel Time Estimation Using Correlation Analysis of Single Loop Detector Data.” In Transportation Research Record: Journal of the Transportation Research Board, No. 1968, pp. 10-19.

Hadi, M., Zhan, C., Alvarez, P. (2010). "Traffic Management Simulation Support.” Final Report, Prepared for Florida Department of Transportation by Lehman Center for Transportation Research, Tallahassee, FL.

Hall, F., Hurdle, V., and Banks, J. (1992). "Synthesis of Recent Work on the Nature of Speed-flow and Flow-occupancy (or Density) Relationships on Freeways." Transportation Research Record: Journal of the Transportation Research Board, No. 1365, pp. 12-18.

Hamad, K., and Faghri, A. (2005). "Hybrid Empirical Mode Decomposition-Neuro Model for Short-Term Travel Time Prediction on Freeways." Transportation Research Board 84th Annual Meeting (CD-ROM), National Academies Press, Washington D.C.

Huiisken, G., and Van Berkum, E. C. (2003). "A Comparative Analysis of Short-Range Travel Time Prediction Methods.” Transportation Research Board 82nd Annual Meeting (CD-ROM), National Academies Press, Washington D.C.

Innamaa, S. (2005). "Short-Term Prediction of Travel Time Using Neural Networks on an Interurban Highway.” Transportation, Vol. 32, pp. 649-669.

Ishak, S. and Al-Deek, H. (2002). "Performance Evaluation of Short-Term Time-Series Traffic Prediction Model." Journal of Transportation Engineering, ASCE, November/December, pp. 490-498.

Ishak, S., Kotha, P., and Alecsandru, C. (2003). "Optimization of Dynamic Neural Network Performance for Short-Term Traffic Prediction." Transportation Research Record: Journal of the Transportation Research Board, No. 1836, pp. 45-56.

Ishak, S., and Alecsandru, C. (2004). "Optimizing Traffic Prediction Performance of Neural Networks under Various Topological, Input, and Traffic Condition Settings." Journal of Transportation Engineering, ASCE, July/August, pp. 452-465. 
Jacobson, L. N., Nihan, N. L., and Bender, J. D. (1990). "Detecting Erroneous Loop Detector Data in a Freeway Traffic Management System.” Transportation Research Record: Journal of the Transportation Research Board, No. 1287, pp. 151-166.

Jain, M., and Coifman, B. (2005). "Improved Speed Estimates from Freeway Traffic Detectors.” Journal of Transportation Engineering, ASCE, July, pp. 483-495.

Jeng, S., Ritchie, S. G., and Tok, Y. C. (2007). "Freeway Corridor Performance Measurement Based on Vehicle Reidentification.” Transportation Research Board 86th Annual Meeting (CD-ROM), National Academies Press, Washington D.C.

Kaneko, Y., Ohe, I., Kawashima, H., and Hirano, T. (1995). "The Judgment of the Traffic Condition by Using the Cluster Analysis." IEEE Vehicle Navigation and Information Systems Conference Proceedings, pp. 218-224.

Kerner, B., and Rehborn, H. (1996). "Experimental Properties of Complexity in Traffic Flow.” Physical Review E., Vol. 53, No. 5, pp. 4275-4278.

Kisgyörgy, L., and Rilett, L. R. (2002). "Travel Time Prediction by Advanced Neural Network.” Periodica Polytechnica Civil Engineering, Vol. 46, No. 1, pp. 15-32.

Kondagari, S. (2006). ” A Probabilistic Approach for Modeling and Real-time Filtering of Freeway Detector Data.” Master Thesis, Louisiana State University, Baton Rouge, LA.

Kothuri, S. M., Tufte, K. A., Hagedorn, H., Bertini, R. L., and Deeter, D. (2007). "Survey of Best Practices in Real Time Travel Time Estimation and Prediction." Compendium of Technical Papers, Institute of Transportation Engineers, District 6 Annual Meeting, Portland, Oregon, July 15-18, 2007.

Kothuri, S. M., Tufte, K. A., Fayed, E., and Bertini, R. L. (2008). "Toward Understanding and Reducing Errors in Real-Time Estimation of Travel Times." Transportation Research Record: Journal of the Transportation Research Board, No. 2049, pp. 21-28.

Kuchipudi, C. M., and Chien, S. I. J. (2003). "Development of a Hybrid Model for Dynamic Travel Time Prediction.” Transportation Research Board 82nd Annual Meeting (CD-ROM), National Academies Press, Washington D.C.

Kury, Y. (2008). "Evaluation of Travel Time Estimates for Dynamic Message Sign (DMS) Display.” Master Project Report. Florida International University.

Kwon, J., Coifman, B., and Bickel, P. (2000). "Day-to-Day Travel Time Trends and Travel Time Prediction from Loop Detector Data." Transportation Research Record: Journal of the Transportation Research Board, No. 1717, pp. 120-129. 
Kwon, J., and Petty, K. (2005). "A Travel Time Prediction Algorithm Scalable to Freeway Networks with Many Nodes with Arbitrary Travel Routes.” Transportation Research Record: Journal of the Transportation Research Board, No. 1935, pp. 147-153.

Lam, S., and Toan, T. D. (2008). "Short-Term Travel Time Prediction Using Support Vector Regression.” Transportation Research Board 87th Annual Meeting (CD-ROM), National Academies Press, Washington D.C.

Li., R., Rose, G.., and Sarvi, M. (2006). "Evaluation of Speed-Based Travel Time Estimation Models.” Journal of Transportation Engineering, ASCE, Vol. 132, No. 7, pp. 540-547.

Li., R., Rose, G.., and Sarvi, M. (2007). "Modelling and Estimation of Travel Time Variability." Transportation Research Board 86th Annual Meeting (CD-ROM), National Academies Press, Washington D.C.

Li., S. (2002). "Nonlinear Combination of Travel-Time Prediction Model Based on Wavelet Network." Proceedings of the IEEE 5th International Conference on Intelligent Transportation Systems, Singapore.

Liu, Y., and Chang, G. (2006). "Estimation of Freeway Travel Time Based on Sparsely Distributed Detectors." Proceedings of Applications of Advanced Technology in Transportation, The Ninth International Conference, American Society of Civil Engineers, pp. 560-565.

Liu, X. Y., and Varaiya, P., Horowitz, R., and Palen, J. (2008). "Faulty Loop Data Analysis /Corection and Loop Fault Detection." Proceedings of 15th ITS World Congress, New York.

Liu, Y., Lin, P., Lai, X., Chang, G., and Marquess, A. (2006). "Developments and Applications of a Simulation-Based Online Travel Time Prediction System for Ocean City, Maryland." Presented at 85th Annual Meeting of Transportation Research Board, Washington, D.C.

Logendran R., and Wang, L. (2008). "Dynamic Travel Time Estimation Using Regression Trees.” Final Report. FHWA-OR-RD-09-09.

Lyman, K., and Bertini, R. L. (2009). "Using Travel Time Reliability Measures to Improve Regional Transportation Planning and Operations." Transportation Research Record: Journal of the Transportation Research Board, No. 2046, pp. 1.

May, D., Cayford, R., Leiman, L., and Merritt, G. (2005). "Berkeley Highway Laboratory Project: Final Report.” California PATH Research Report, UCB-ITS-PRR-2005-24. 
Middleton, D., and Parker, R. (2002). "Vehicle Detector Evaluation." Report, FHWA/TX-03/2119-1.

Miska, M. P., Muller, T. H. J., and Zuylen, H. J. (2005). “Online Travel Time Prediction with Real-time Microscopic Simulation.” Transportation Research Board 84th Annual Meeting (CD-ROM), National Academies Press, Washington D.C.

Meehan, B. (2005). “Travel Times on Dynamic Message Signs.” ITE Journal, September.

Nam D. H., and Drew, D. R. (1996). “Traffic Dynamics: Method for Estimating Freeway Travel Times in Real Time from Flow Measurement.” Journal of Transportation Engineering. ASCE, May/June, pp. 185-191.

Nam D. H., and Drew, D. R. (1999). “Automatic Measurement of Traffic Variables for Intelligent Transportation Systems Applications.” Transportation Research Part B, Vol. 33, pp. 437-457.

Nguyen, L. N., and Scherer, W. T. (2003). "Imputation Techniques to Account for Missing Data in Support of Intelligent Transportation Systems Applications.” Technical Report, UVACTS-13-0-78.

Ni D., Leonard II, J. D., Guin, A., and Feng, C. (2005). "Multiple Imputation Scheme for Overcoming the Missing Values and Variability Issues in ITS Data." Journal of Transportation Engineering, ASCE, December, pp. 931-938.

Nihan, N., Jacobson, L. N., Bender, J. D., and Davis, G. (1990). “Detector Data Validity.” Final Report, WA-RD 208.1.

Ndoye, M., Totten, V., Carter, B., Bullock, D. M., and Krogmeier, J. V. (2008). "Vehicle Detector Signature Processing and Vehicle Re-identification for Travel Time Estimation." Transportation Research Board 87th Annual Meeting (CD-ROM), National Academies Press, Washington D.C.

Oh, J., and Chung, Y. (2006). “Calculation of Travel Time Variability from Loop Detector Data." Transportation Research Record: Journal of the Transportation Research Board, No. 1945, pp. 12-23.

Oh, C., and Park, S. (2008). "Investigating the Effects of Travel Time Patterns on Predictability." Transportation Research Board 87th Annual Meeting (CD-ROM), National Academies Press, Washington D.C.

Park, D., and Rilett, L. R. (1998). "Forecasting Multiple-Period Freeway Link Travel Times Using Modular Neural Networks.” Transportation Research Record: Journal of the Transportation Research Board, No. 1617, pp. 163-170. 
Park, D., and Rilett, L. R. (1999). "Forecasting Freeway Link Travel Times with a Multilayer Feedforward Neural Network.” Computer-Aided Civil and Infrastructure Engineering, Vol. 14, pp. 357-367.

Park, D., Rilett, L. R., and Han, G. (1999). "Spectral Basis Neural Networks for Real-Time Travel Time Forecasting.” Journal of Transportation Engineering, ASCE, November/December, pp. 515-523.

Payne, H. J., Helfenbein, E. D., Knobel, H. C. (1976). "Development and Testing of Incident Detection Algorithms.” Technical Report, FHWA-RD-76-20, Federal Highway Administration, Washington D.C.

Petty, K., Bickel, P., Jiang, J., Ostland, M., Rice, J., Ritov, Y., and Schoenberg, F. (1998). "Accurate Estimation of Travel Times from Single-loop Detectors." Transportation Research, Part B: Methodological, Vol. 32, No. 1, pp. 1-17.

Quiroga, C., Hamad, K., and Park, E. S. (2005). “Incident Detection Optimization and Data Quality Control.” Technical Report, FHWA/TX-06/0-4745-3.

Rice, J., and Zwet, E. (2004). “A Simple and Effective Method for Predicting Travel Times on Freeways.” IEEE Transaction on Intelligent Transportation Systems, Vol. 5, No. 3, pp. 200-207.

Rilett, L. R., and Park, D. (1999). "Direct Forecasting of Freeway Corridor Travel Times Using Spectral Basis Neural Networks.” Transportation Research Record: Journal of the Transportation Research Board, No. 1752, pp. 140-147.

Ritchie, S. G., Park, S., Oh, C., Jeng, S., and Tok, A. (2005). “Anonymous Vehicle Tracking for Real-Time Freeway and Arterial Street Performance Measurement." California PATH Research Report, UCB-ITS-PRR-2005-9.

Roess, R. P., Prassas, E. S., and McShane, W. R. (2004). “Traffic Engineering”, Third Edition. Peason Prentice Hall, New Jersey.

Savran, A. (2007). "Multifeedback-Layer Neural Network.” IEEE Transaction on Neural Networks, Vol. 18, No. 2, pp.373-384.

Shen, L. (2008). ” Freeway Travel Time Prediction System Using Dynamic Neural Networks.” Ph.D. Dissertation, Florida International University, Miami, FL.

Sun, H., Liu, H. X., Xiao, H., He, R.R., and Ran, B. (2003). "Short-Term Traffic Forecasting Using the Local Linear Regression Model.” Transportation Research Record: Journal of the Transportation Research Board, No. 1836, pp. 143-150. 
Sun, Y., Liu, H., and Zhang, K. (2008). "Travel Time Prediction Based on Fuzzy Logic Data Fusion Method.” 15th World Congress on Intelligent Transport Systems, New York, NY.

Tao, Y., Yang, F., Qiu, Z., and Ran, B. (2006). "Travel Time Prediction in the Presence of Traffic Incidents Using Different Types of Neural Networks.” Transportation Research Board 85th Annual Meeting (CD-ROM), National Academies Press, Washington D.C.

Tu, H., Van Lint, H., and Van Zuylen, H. (2006). "Travel Time Variability versus Freeway Characteristics." Proceedings of the 2006 IEEE Intelligent Transportation Systems Conference, Toronto, Canada.

Turner, S. M., Eisele, W. L., Benz, R. J., and Holdener, D. J. (1998). "Travel Time Data Collection Handbook.” Report FHWA-PL-98-035.

Turner. S., Magiotta, R., and Lonax, T. (2004). "Monitoring Urban Freeways in 2003: Current Conditions and Trends from Archived Operations Data." Final Report. FHWA-HOP-05-018.

Turner, S. (2007). "Quality Control Procedures for Archived Operations Traffic Data Synthesis of Practice and Recommendations.” Technical Report.

University of Florida Transportation Research Center. (2005). "Central Data Warehouse Quality Control Considerations.” Draft Working Paper BD547-37-1.

Van Hinsbergen, C. P. IJ., and Van Lint, J. W. C. (2008). "Bayesian Combination of Travel Time Prediction Models." Transportation Research Record: Journal of the Transportation Research Board, No. 2064(-1), pp. 73-80.

Van Lint, J. W. C., Hoogendoorn, S. P., and Van Zuylen, H. J. (2002). "Freeway Travel Time Prediction with State-Space Neural Networks: Modeling State-Space Dynamics with Recurrent Neural Networks." Transportation Research Record: Journal of the Transportation Research Board, No. 1811, pp. 30-39.

Van Lint, J. W. C. (2004). "Reliable Freeway Travel Time Estimation.” Ph.D. Dissertation. Delft University of Technology, Netherlands.

Van Lint, J. W. C. (2004). "Quantifying Uncertainty in Real-Time Neural Network Based Freeway Travel Prediction: Practical Confidence and Prediction Intervals for ATIS.” Transportation Research Board 83rd Annual Meeting (CD-ROM), National Academies Press, Washington D.C.

Van Lint, J. W. C., Hoogendoorn, S. P., and Van Zuylen, H. J. (2005). “Accurate Freeway Travel Time Prediction with State-Space Neural Networks under Missing Data." Transportation Research Part C, Vol. 13, pp. 347-369. 
Van Lint, J. W. C. (2006). "Reliable Real-Time Framework for Short-Term Freeway Travel Time Prediction.” Journal of Transportation Engineering, ASCE, December, pp.921-932.

Vanajakshi, L. D. (2004). "Estimation and Prediction of Travel Time from Loop Detector Data for Intelligent Transportation Systems Applications.” Ph.D. Dissertation. Texas A\&M University, Texas.

Vanajakshi, L. D, Subramanian, S. C., and Sivanandan, R. (2008). “Short Term Prediction of Travel Time for Indian Traffic Conditions Using Buses as Probe Vehicles." Transportation Research Board 87th Annual Meeting (CD-ROM), National Academies Press, Washington D.C.

Vanajakshi, L. D, Williams, B. M., and Rilett, L. R. (2009). “Improved Flow-Based Travel Time Estimation Method from Point Detector Data for Freeways.” Journal of Transportation Engineering. ASCE, Vol. 135, No. 1, pp. 475-483.

Viti, F., Verbeke, W., and Tampère, C. M. J. (2008). "Sensor Locations for Reliable Travel Time Prediction and Dynamic Management of Traffic Networks." Transportation Research Record: Journal of the Transportation Research Board, No. 2049, pp. 103-110.

Waller, S. T., Chiu, Y., Ruizjuri, N., Unnikrishnan, A., and Bustillos, B. (2007). "Short Term Travel Time Prediction on Freeways in Conjunction with Detector Coverage Analysis.” Technical Report, FHWA/TX-08/0-5141-1.

Wang, J., Zou, N., and Chang, G. (2008). "Travel Time Prediction - Empirical Analysis of Missing Data Issues for Advanced Traveler Information System Application.” Transportation Research Record: Journal of the Transportation Research Board, No. 2049, pp. 81-91.

Wen, Y., Lee, T., and Cho, H. (2005). "Missing Data Treatment and Data Fusion Toward Travel Time Estimation for ATIS.” Journal of the Eastern Asia Society for Transportation Studies, Vol. 6, pp. 2546-2560.

Williams, B. M., and Hoel, L. A. (2003). "Modeling and Forecasting Vehicular Traffic Flow as a Seasonal ARIMA Process: Theoretical Basis and Empirical Results.” Journal of Transportation Engineering, November/December, pp. 664-672.

Wu, C., Ho, J., and Lee, D. T. (2004). “Travel-Time Prediction with Support Vector Regression.” IEEE Transactions on Intelligent Transportation Systems, Vol. 5, No. 4, pp. 276-281.

Wunderlich, K. E., Kaufman, D. E., and Smith, R. L. (2000). "Link Travel Time Prediction for Decentralized Route Guidance Architectures.” IEEE Transactions on Intelligent Transportation Systems, Vol. 1, No. 1, pp. 4-14. 
Wunnava, S., Yen, K., Babij, T., Zavaleta, R., Romero, R., and Archillar, C. (2007). “Travel Time Estimation Using Cell Phones (TTECP) for Highways and Roadways." Contract BD015-12.

Xia, J., and Chen, M. (2007). “Freeway Travel Time Forecasting Under Incident.” Final Report.

Yeon, J., and Ko, B. (2007). "Comparison of Travel Time Estimation Using Shockwave Analysis and Queuing Theory to Field Data along Freeways.” 2007 International Conference on Multimedia and Ubiquitous Engineering (MUE'07).

Yi, T. (2009). “Travel Time Estimation from Fixed Point Detector Data.” Ph.D. Dissertation, North Carolina State University, Raleigh, North Carolina.

You, J., and Kim, T. J. (2000). "Development and Evaluation of a Hybrid Travel Time Forecasting Model.” Transportation Research Part C, Vol. 8, pp. 231-256.

Zhang, L., and Levinson, D. (2004). "Some Properties of Flows at Freeway Bottleneck.” Transportation Research Board 83rd Annual Meeting (CD-ROM), National Academies Press, Washington D.C.

Zhang, W. (2006). "Freeway Travel Time Estimation Based on Spot Speed Measurements.” Ph. D. Dissertation. Virginia Polytechnic Institute and State University, Blacksburg, Virginia.

Zhang, X., J.A. Rice. Short-Term Travel Time Prediction. Transportation Research Part C, Vol. 11, 2003, pp.187-210.

Zheng, W., Lee, D., and Shi, Q. (2006). “Short-Term Freeway Traffic Flow Prediction: Bayesian Combined Neural Network Approach.” Journal of Transportation Engineering, ASCE, Vol. 132, No. 2, pp.114-121.

Zhong, M., Sharama, S., and Lingras, P. (2006). "Matching Patterns for Updating Missing Values of Traffic Counts.” Transportation Planning and Technology, Vol. 29, No. 


\section{APPENDIX A \\ SENSITIVITY ANALYSIS RESULTS FOR TRAVEL TIME ESTIMATION}

This appendix presents detailed analysis results for the impacts of major influential factors on the accuracy and reliability of travel time estimates.

\section{A.1 Impacts of Data Smoothing Methods}

Table A-1 Accuracy and Reliability of Travel Time Estimation Using Simple Moving Average

\begin{tabular}{|l|c|c|c|c|c|c|}
\hline \multirow{4}{*}{ Method } & $\begin{array}{c}\text { Rolling } \\
\text { Period }\end{array}$ & $\begin{array}{c}\text { MAE } \\
\mathbf{( M i n . )}\end{array}$ & $\begin{array}{c}\text { MAPE } \\
\mathbf{( \% )}\end{array}$ & $\begin{array}{c}\text { Reliability } \\
\mathbf{( \% )}\end{array}$ & \% Early & \% Late \\
\hline \multirow{4}{*}{\begin{tabular}{l} 
Moint-to-Point \\
\cline { 2 - 7 }
\end{tabular}} & 1-minute & 1.87 & 14.91 & 58.42 & 3.50 & 38.08 \\
\cline { 2 - 7 } & 2-minute & 1.96 & 15.51 & 58.53 & 2.53 & 38.94 \\
\cline { 2 - 7 } & 3-minute & 2.05 & 16.23 & 59.56 & 2.01 & 38.43 \\
\cline { 2 - 7 } & 4-minute & 2.11 & 16.63 & 59.10 & 2.47 & 38.43 \\
\cline { 2 - 7 } Mid-Point & 5-minute & 2.17 & 17.09 & 58.24 & 3.91 & 37.85 \\
\hline \multirow{5}{*}{ Hethod } & 1-minute & 1.69 & 13.70 & 62.03 & 4.19 & 33.77 \\
\cline { 2 - 7 } & 2-minute & 1.79 & 14.37 & 60.89 & 4.19 & 34.92 \\
\cline { 2 - 7 } & 3-minute & 1.87 & 15.05 & 60.25 & 3.68 & 36.07 \\
\cline { 2 - 7 } & 4-minute & 1.95 & 15.62 & 61.34 & 2.99 & 35.67 \\
\cline { 2 - 7 } & 5-minute & 2.04 & 16.31 & 57.50 & 6.43 & 36.07 \\
\hline & 1-minute & 1.49 & 13.54 & 66.57 & 14.36 & 19.07 \\
\cline { 2 - 7 } & 2-minute & 1.81 & 15.78 & 56.86 & 18.15 & 24.99 \\
\cline { 2 - 7 } & 3-minute & 1.78 & 15.66 & 57.21 & 17.81 & 24.99 \\
\cline { 2 - 7 } & 4-minute & 1.81 & 16.03 & 54.22 & 20.79 & 24.99 \\
\cline { 2 - 7 } & 5-minute & 1.91 & 17.08 & 52.79 & 22.23 & 24.99 \\
\hline \multirow{5}{*}{ Hybrid Model 2 2} & 1-minute & 1.44 & 12.72 & 63.70 & 17.17 & 19.13 \\
\cline { 2 - 7 } & 2-minute & 1.59 & 13.86 & 61.45 & 16.72 & 21.83 \\
\cline { 2 - 7 } & 3-minute & 1.73 & 15.00 & 58.93 & 16.72 & 24.35 \\
\cline { 2 - 7 } & 4-minute & 1.89 & 16.29 & 56.23 & 19.01 & 24.76 \\
\cline { 2 - 7 } & 5-minute & 2.04 & 17.72 & 48.65 & 24.81 & 26.54 \\
\hline
\end{tabular}


Table A-2 Accuracy and Reliability of Travel Time Estimation Using Exponential Moving Average

\begin{tabular}{|c|c|c|c|c|c|c|}
\hline Method & $\begin{array}{c}\text { Smoothing } \\
\text { Factor }\end{array}$ & $\begin{array}{l}\text { MAE } \\
\text { (Min.) }\end{array}$ & $\begin{array}{c}\text { MAPE } \\
(\%)\end{array}$ & $\begin{array}{c}\text { Reliability } \\
\text { (\%) }\end{array}$ & \% Early & \% Late \\
\hline \multirow{5}{*}{$\begin{array}{l}\text { Point-to-Point } \\
\text { Method }\end{array}$} & 0.2 & 2.22 & 17.30 & 57.38 & 1.09 & 41.53 \\
\hline & 0.4 & 2.07 & 16.28 & 57.32 & 1.78 & 40.90 \\
\hline & 0.6 & 2.03 & 16.06 & 58.47 & 2.24 & 39.29 \\
\hline & 0.8 & 2.04 & 16.09 & 58.24 & 2.24 & 39.52 \\
\hline & 1.0 & 2.04 & 16.17 & 55.66 & 3.45 & 40.90 \\
\hline \multirow{5}{*}{$\begin{array}{l}\text { Mid-Point } \\
\text { Method }\end{array}$} & 0.2 & 2.02 & 15.93 & 58.53 & 3.67 & 37.79 \\
\hline & 0.4 & 1.86 & 14.78 & 59.05 & 4.19 & 36.76 \\
\hline & 0.6 & 1.82 & 14.49 & 59.97 & 3.10 & 36.93 \\
\hline & 0.8 & 1.82 & 14.53 & 58.53 & 3.91 & 37.57 \\
\hline & 1.0 & 1.84 & 14.66 & 59.10 & 3.85 & 37.05 \\
\hline \multirow{5}{*}{ Hybrid Model 1} & 0.2 & 1.50 & 13.51 & 61.63 & 12.52 & 25.85 \\
\hline & 0.4 & 1.11 & 10.24 & 70.59 & 10.34 & 19.07 \\
\hline & 0.6 & 1.13 & 10.72 & 66.63 & 14.70 & 18.67 \\
\hline & 0.8 & 1.11 & 10.52 & 66.97 & 14.88 & 18.15 \\
\hline & 1.0 & 1.44 & 12.77 & 61.00 & 19.30 & 19.70 \\
\hline \multirow{5}{*}{ Hybrid Model 2} & 0.2 & 1.49 & 12.51 & 69.21 & 3.33 & 27.46 \\
\hline & 0.4 & 1.15 & 10.12 & 74.50 & 3.33 & 22.17 \\
\hline & 0.6 & 1.03 & 9.28 & 73.92 & 4.36 & 21.71 \\
\hline & 0.8 & 0.99 & 8.98 & 74.73 & 4.54 & 20.74 \\
\hline & 1.0 & 1.09 & 9.60 & 73.23 & 4.48 & 22.29 \\
\hline
\end{tabular}

\section{A.2 Impacts of Data Imputation Methods}

Table A-3 Results of Different Data Imputation Methods without Within-Station Imputation

\begin{tabular}{|l|l|l|c|c|c|c|c|}
\hline \multirow{2}{*}{ Method } & $\begin{array}{l}\text { Temporal } \\
\text { Imputation }\end{array}$ & $\begin{array}{l}\text { Between-Station } \\
\text { Imputation }\end{array}$ & $\begin{array}{l}\text { MAE } \\
\text { (Min.) }\end{array}$ & $\begin{array}{c}\text { MAPE } \\
\mathbf{( \% )}\end{array}$ & $\begin{array}{c}\text { Reliability } \\
\mathbf{( \% )}\end{array}$ & $\begin{array}{c}\text { \% } \\
\text { Early }\end{array}$ & $\begin{array}{c}\text { \% } \\
\text { Late }\end{array}$ \\
\hline $\begin{array}{l}\text { Point-to- } \\
\text { Point } \\
\text { Method }\end{array}$ & $\begin{array}{l}\text { W/o } \\
\text { Temporal } \\
\text { Imputation }\end{array}$ & Simple Average & 2.09 & 16.38 & 57.09 & 2.93 & 39.98 \\
\cline { 3 - 8 } & Linear Interpolation & 2.09 & 16.35 & 57.09 & 2.93 & 39.98 \\
\cline { 2 - 8 } & Factor Method & 2.09 & 16.38 & 57.15 & 3.10 & 39.75 \\
\hline
\end{tabular}




\begin{tabular}{|c|c|c|c|c|c|c|c|}
\hline Method & $\begin{array}{l}\text { Temporal } \\
\text { Imputation }\end{array}$ & $\begin{array}{l}\text { Between-Station } \\
\text { Imputation }\end{array}$ & $\begin{array}{l}\text { MAE } \\
\text { (Min.) }\end{array}$ & $\begin{array}{c}\text { MAPE } \\
(\%)\end{array}$ & $\begin{array}{c}\text { Reliability } \\
(\%)\end{array}$ & $\begin{array}{c}\% \\
\text { Early }\end{array}$ & $\begin{array}{c}\% \\
\text { Late }\end{array}$ \\
\hline \multirow{3}{*}{$\begin{array}{l}\text { Point-to- } \\
\text { Point } \\
\text { Method }\end{array}$} & \multirow{3}{*}{$\begin{array}{l}\text { Average of } \\
\text { Temporal } \\
\text { and Spatial } \\
\text { Imputations }\end{array}$} & Simple Average & 2.08 & 16.28 & 57.09 & 2.93 & 39.98 \\
\hline & & Linear Interpolation & 2.08 & 16.25 & 57.09 & 2.93 & 39.98 \\
\hline & & Factor Method & 2.08 & 16.30 & 57.09 & 2.93 & 39.98 \\
\hline \multirow{6}{*}{$\begin{array}{l}\text { Mid-Point } \\
\text { Method }\end{array}$} & \multirow{3}{*}{$\begin{array}{l}\text { w/o } \\
\text { Temporal } \\
\text { Imputation }\end{array}$} & Simple Average & 1.87 & 14.86 & 59.97 & 3.50 & 36.53 \\
\hline & & Linear Interpolation & 1.87 & 14.85 & 59.97 & 3.50 & 36.53 \\
\hline & & Factor Method & 1.88 & 14.95 & 59.74 & 3.50 & 36.76 \\
\hline & \multirow{3}{*}{\begin{tabular}{|l|} 
Average of \\
Temporal \\
and Spatial \\
Imputations
\end{tabular}} & Simple Average & 1.86 & 14.79 & 59.97 & 3.50 & 36.53 \\
\hline & & Linear Interpolation & 1.86 & 14.77 & 59.97 & 3.50 & 36.53 \\
\hline & & Factor Method & 1.87 & 14.81 & 59.97 & 3.50 & 36.53 \\
\hline \multirow{8}{*}{$\begin{array}{l}\text { Hybrid } \\
\text { Model } 1\end{array}$} & \multirow{4}{*}{$\begin{array}{l}\text { w/o } \\
\text { Temporal } \\
\text { Imputation }\end{array}$} & Simple Average & 1.09 & 10.23 & 68.70 & 10.91 & 20.39 \\
\hline & & \begin{tabular}{|l} 
Linear Interpolation \\
\end{tabular} & 1.08 & 10.16 & 68.70 & 10.91 & 20.39 \\
\hline & & $\begin{array}{l}\text { Linear Interpolation for } \\
\text { Speed and Occupancy, } \\
\text { and Factor for Volume } \\
\end{array}$ & 1.10 & 10.35 & 68.75 & 10.68 & 20.56 \\
\hline & & Factor Method & 1.15 & 10.79 & 66.28 & 12.92 & 20.79 \\
\hline & \multirow{4}{*}{$\begin{array}{l}\text { Average of } \\
\text { Temporal } \\
\text { and Spatial } \\
\text { Imputations }\end{array}$} & Simple Average & 1.08 & 10.15 & 68.70 & 10.91 & 20.39 \\
\hline & & Linear Interpolation & 1.08 & 10.09 & 68.70 & 10.91 & 20.39 \\
\hline & & $\begin{array}{l}\text { Linear Interpolation for } \\
\text { Speed and Occupancy, } \\
\text { and Factor for Volume } \\
\end{array}$ & 1.09 & 10.20 & 68.70 & 10.91 & 20.39 \\
\hline & & Factor Method & 1.11 & 10.46 & 68.70 & 10.91 & 20.39 \\
\hline \multirow{8}{*}{$\begin{array}{l}\text { Hybrid } \\
\text { Model } 2\end{array}$} & \multirow{4}{*}{$\begin{array}{l}\text { w/o } \\
\text { Temporal } \\
\text { Imputation }\end{array}$} & Simple Average & 1.23 & 10.72 & 72.20 & 4.77 & 23.03 \\
\hline & & \begin{tabular}{|l|} 
Linear Interpolation \\
\end{tabular} & 1.23 & 10.70 & 72.20 & 4.77 & 23.03 \\
\hline & & $\begin{array}{l}\text { Linear Interpolation for } \\
\text { Speed and Occupancy, } \\
\text { and Factor for Volume } \\
\end{array}$ & 1.23 & 10.70 & 72.20 & 4.77 & 23.03 \\
\hline & & Factor Method & 1.24 & 10.77 & 71.97 & 4.77 & 23.26 \\
\hline & \multirow{4}{*}{$\begin{array}{l}\text { Average of } \\
\text { Temporal } \\
\text { and Spatial } \\
\text { Imputations }\end{array}$} & Simple Average & 1.23 & 10.64 & 72.20 & 4.77 & 23.03 \\
\hline & & Linear Interpolation & 1.22 & 10.61 & 72.20 & 4.77 & 23.03 \\
\hline & & $\begin{array}{l}\text { Linear Interpolation for } \\
\text { Speed and Occupancy, } \\
\text { and Factor for Volume }\end{array}$ & 1.22 & 10.61 & 72.20 & 4.77 & 23.03 \\
\hline & & Factor Method & 1.23 & 10.64 & 72.20 & 4.77 & 23.03 \\
\hline
\end{tabular}


Table A-4 Results of Different Data Imputation Methods with Within-station Imputation

\begin{tabular}{|c|c|c|c|c|c|c|c|}
\hline Method & $\begin{array}{l}\text { Temporal } \\
\text { Imputation }\end{array}$ & $\begin{array}{l}\text { Between-Station } \\
\text { Imputation }\end{array}$ & $\begin{array}{l}\text { MAE } \\
\text { (Min.) }\end{array}$ & $\begin{array}{c}\text { MAPE } \\
(\%)\end{array}$ & $\begin{array}{c}\text { Reliability } \\
\text { (\%) }\end{array}$ & $\begin{array}{c}\% \\
\text { Early }\end{array}$ & $\begin{array}{c}\% \\
\text { Late }\end{array}$ \\
\hline \multirow{6}{*}{$\begin{array}{l}\text { Point-to- } \\
\text { Point } \\
\text { Method }\end{array}$} & \multirow{3}{*}{$\begin{array}{l}\text { w/o } \\
\text { Temporal } \\
\text { Imputation }\end{array}$} & Simple Average & 2.06 & 16.14 & 57.09 & 2.93 & 39.98 \\
\hline & & Linear Interpolation & 2.06 & 16.14 & 57.09 & 2.93 & 39.98 \\
\hline & & Factor Method & 2.06 & 16.14 & 57.09 & 2.93 & 39.98 \\
\hline & \multirow{3}{*}{\begin{tabular}{|l} 
Average of \\
Temporal \\
and Spatial \\
Imputations \\
\end{tabular}} & Simple Average & 2.06 & 16.14 & 57.09 & 2.93 & 39.98 \\
\hline & & Linear Interpolation & 2.06 & 16.14 & 57.09 & 2.93 & 39.98 \\
\hline & & Factor Method & 2.06 & 16.14 & 57.09 & 2.93 & 39.98 \\
\hline \multirow{6}{*}{$\begin{array}{l}\text { Mid-Point } \\
\text { Method }\end{array}$} & \multirow{3}{*}{$\begin{array}{l}\text { w/o } \\
\text { Temporal } \\
\text { Imputation }\end{array}$} & Simple Average & 1.84 & 14.66 & 59.97 & 3.50 & 36.53 \\
\hline & & Linear Interpolation & 1.84 & 14.66 & 59.97 & 3.50 & 36.53 \\
\hline & & Factor Method & 1.84 & 14.66 & 59.97 & 3.50 & 36.53 \\
\hline & \multirow{3}{*}{$\begin{array}{l}\text { Average of } \\
\text { Temporal } \\
\text { and Spatial } \\
\text { Imputations } \\
\end{array}$} & Simple Average & 1.84 & 14.66 & 59.97 & 3.50 & 36.53 \\
\hline & & Linear Interpolation & 1.84 & 14.66 & 59.97 & 3.50 & 36.53 \\
\hline & & Factor Method & 1.84 & 14.66 & 59.97 & 3.50 & 36.53 \\
\hline \multirow{8}{*}{$\begin{array}{l}\text { Hybrid } \\
\text { Model } 1\end{array}$} & \multirow{4}{*}{$\begin{array}{l}\text { w/o } \\
\text { Temporal } \\
\text { Imputation }\end{array}$} & Simple Average & 1.13 & 10.49 & 68.52 & 10.91 & 20.56 \\
\hline & & Linear Interpolation & 1.12 & 10.41 & 68.70 & 10.91 & 20.39 \\
\hline & & $\begin{array}{l}\text { Linear Interpolation for } \\
\text { Speed and Occupancy, } \\
\text { and Factor for Volume } \\
\end{array}$ & 1.14 & 10.61 & 68.52 & 10.91 & 20.56 \\
\hline & & Factor Method & 1.14 & 10.72 & 68.52 & 10.68 & 20.79 \\
\hline & \multirow{4}{*}{$\begin{array}{l}\text { Average of } \\
\text { Temporal } \\
\text { and Spatial } \\
\text { Imputations }\end{array}$} & Simple Average & 1.14 & 10.53 & 68.52 & 10.91 & 20.56 \\
\hline & & Linear Interpolation & 1.13 & 10.48 & 68.52 & 10.91 & 20.56 \\
\hline & & $\begin{array}{l}\text { Linear Interpolation for } \\
\text { Speed and Occupancy, } \\
\text { and Factor for Volume } \\
\end{array}$ & 1.14 & 10.59 & 68.52 & 10.91 & 20.56 \\
\hline & & Factor Method & 1.17 & 10.84 & 67.15 & 10.91 & 21.94 \\
\hline \multirow{4}{*}{$\begin{array}{l}\text { Hybrid } \\
\text { Model } 2\end{array}$} & \multirow{4}{*}{$\begin{array}{l}\text { w/o } \\
\text { Temporal } \\
\text { Imputation }\end{array}$} & Simple Average & 1.20 & 10.48 & 73.58 & 4.77 & 21.65 \\
\hline & & Linear Interpolation & 1.20 & 10.48 & 73.58 & 4.77 & 21.65 \\
\hline & & $\begin{array}{l}\text { Linear Interpolation for } \\
\text { Speed and Occupancy, } \\
\text { and Factor for Volume } \\
\end{array}$ & 1.20 & 10.48 & 73.58 & 4.77 & 21.65 \\
\hline & & Factor Method & 1.20 & 10.48 & 73.58 & 4.77 & 21.65 \\
\hline
\end{tabular}




\begin{tabular}{|c|c|c|c|c|c|c|c|}
\hline Method & $\begin{array}{l}\text { Temporal } \\
\text { Imputation }\end{array}$ & $\begin{array}{l}\text { Between-Station } \\
\text { Imputation }\end{array}$ & $\begin{array}{l}\text { MAE } \\
\text { (Min.) }\end{array}$ & $\begin{array}{c}\text { MAPE } \\
(\%)\end{array}$ & $\begin{array}{c}\text { Reliability } \\
\text { (\%) }\end{array}$ & $\begin{array}{c}\% \\
\text { Early }\end{array}$ & $\begin{array}{c}\% \\
\text { Late }\end{array}$ \\
\hline \multirow{4}{*}{$\begin{array}{l}\text { Hybrid } \\
\text { Model } 2\end{array}$} & \multirow{4}{*}{$\begin{array}{l}\text { Average of } \\
\text { Temporal } \\
\text { and Spatial } \\
\text { Imputations }\end{array}$} & Simple Average & 1.20 & 10.48 & 73.58 & 4.77 & 21.65 \\
\hline & & Linear Interpolation & 1.20 & 10.48 & 73.58 & 4.77 & 21.65 \\
\hline & & $\begin{array}{l}\text { Linear Interpolation for } \\
\text { Speed and Occupancy, } \\
\text { and Factor for Volume }\end{array}$ & 1.20 & 10.48 & 73.58 & 4.77 & 21.65 \\
\hline & & Factor Method & 1.20 & 10.48 & 73.58 & 4.77 & 21.65 \\
\hline
\end{tabular}

\section{A.3 Impacts of Intrinsic Errors}

Table A-5 Impacts of Intrinsic Errors on Travel Time Estimation Performance for Simulated Uncongested Conditions

\begin{tabular}{|c|c|c|c|c|c|c|c|}
\hline Method & \multicolumn{2}{|c|}{ Cases } & $\begin{array}{l}\text { MAE } \\
\text { (Min.) }\end{array}$ & $\begin{array}{c}\text { MAPE } \\
(\%)\end{array}$ & $\begin{array}{c}\text { Reliability } \\
\text { (\%) }\end{array}$ & $\begin{array}{c}\% \\
\text { Early }\end{array}$ & $\%$ Late \\
\hline \multirow{4}{*}{$\begin{array}{l}\text { Point-to-Point } \\
\text { Method }\end{array}$} & \multicolumn{2}{|c|}{ w/o Errors } & 0.121 & 1.92 & 100 & 0 & 0 \\
\hline & \multirow{3}{*}{$\begin{array}{c}\text { w/ } \\
\text { Intrinsic } \\
\text { Errors }\end{array}$} & Average & 0.123 & 1.95 & 100 & 0 & 0 \\
\hline & & Minimum & 0.121 & 1.92 & 100 & 0 & 0 \\
\hline & & Maximum & 0.125 & 1.98 & 100 & 0 & 0 \\
\hline \multirow{4}{*}{$\begin{array}{l}\text { Mid-Point } \\
\text { Method }\end{array}$} & \multicolumn{2}{|c|}{ w/o Errors } & 0.082 & 1.31 & 100 & 0 & 0 \\
\hline & \multirow{3}{*}{$\begin{array}{c}\text { w/ } \\
\text { Intrinsic } \\
\text { Errors }\end{array}$} & Average & 0.083 & 1.33 & 100 & 0 & 0 \\
\hline & & Minimum & 0.081 & 1.30 & 100 & 0 & 0 \\
\hline & & Maximum & 0.085 & 1.36 & 100 & 0 & 0 \\
\hline \multirow{4}{*}{ Hybrid Model 1} & \multicolumn{2}{|c|}{ w/o Errors } & 0.082 & 1.31 & 100 & 0 & 0 \\
\hline & \multirow{3}{*}{$\begin{array}{c}\text { w/ } \\
\text { Intrinsic } \\
\text { Errors }\end{array}$} & Average & 0.083 & 1.33 & 100 & 0 & 0 \\
\hline & & Minimum & 0.081 & 1.3 & 100 & 0 & 0 \\
\hline & & Maximum & 0.085 & 1.36 & 100 & 0 & 0 \\
\hline \multirow{4}{*}{ Hybrid Model 2} & \multicolumn{2}{|c|}{ w/o Errors } & 0.082 & 1.31 & 100 & 0 & 0 \\
\hline & \multirow{3}{*}{$\begin{array}{c}\text { w/ } \\
\text { Intrinsic } \\
\text { Errors }\end{array}$} & Average & 0.083 & 1.33 & 100 & 0 & 0 \\
\hline & & Minimum & 0.081 & 1.30 & 100 & 0 & 0 \\
\hline & & Maximum & 0.085 & 1.36 & 100 & 0 & 0 \\
\hline
\end{tabular}


Table A-6 Impacts of Intrinsic Errors on Travel Time Estimation Performance for Simulated Incident Case 1 between 7:30 A.M. and 8:30 A.M.

\begin{tabular}{|c|c|c|c|c|c|c|c|}
\hline Method & \multicolumn{2}{|c|}{ Cases } & $\begin{array}{l}\text { MAE } \\
\text { (Min.) }\end{array}$ & $\begin{array}{c}\text { MAPE } \\
(\%)\end{array}$ & $\begin{array}{c}\text { Reliability } \\
\text { (\%) }\end{array}$ & $\begin{array}{c}\% \\
\text { Early }\end{array}$ & $\%$ Late \\
\hline \multirow{4}{*}{$\begin{array}{l}\text { Point-to-Point } \\
\text { Method }\end{array}$} & \multicolumn{2}{|c|}{ w/o Errors } & 2.07 & 16.28 & 57.32 & 1.78 & 40.90 \\
\hline & \multirow{3}{*}{$\begin{array}{c}\mathrm{w} / \\
\text { Intrinsic } \\
\text { Errors }\end{array}$} & Average & 2.03 & 16.02 & 57.84 & 2.00 & 40.16 \\
\hline & & Minimum & 1.96 & 15.43 & 54.16 & 1.72 & 38.43 \\
\hline & & Maximum & 2.13 & 16.73 & 59.62 & 3.04 & 44.06 \\
\hline \multirow{4}{*}{$\begin{array}{l}\text { Mid-Point } \\
\text { Method }\end{array}$} & \multicolumn{2}{|c|}{ w/o Errors } & 1.86 & 14.78 & 59.05 & 4.19 & 36.76 \\
\hline & \multirow{3}{*}{\begin{tabular}{|c|}
$\mathrm{w} /$ \\
Intrinsic \\
Errors
\end{tabular}} & Average & 1.83 & 14.59 & 60.24 & 3.95 & 35.81 \\
\hline & & Minimum & 1.76 & 14.08 & 58.24 & 3.10 & 34.18 \\
\hline & & Maximum & 1.91 & 15.15 & 62.26 & 4.77 & 37.79 \\
\hline \multirow{4}{*}{ Hybrid Model 1} & \multicolumn{2}{|c|}{ w/o Errors } & 1.11 & 10.24 & 70.59 & 10.34 & 19.07 \\
\hline & \multirow{3}{*}{\begin{tabular}{|c|}
$\mathrm{w} /$ \\
Intrinsic \\
Errors
\end{tabular}} & Average & 1.62 & 13.59 & 63.18 & 10.24 & 26.59 \\
\hline & & Minimum & 1.27 & 11.00 & 59.51 & 3.91 & 21.94 \\
\hline & & Maximum & 2.00 & 16.03 & 69.33 & 14.36 & 30.90 \\
\hline \multirow{4}{*}{ Hybrid Model 2} & \multicolumn{2}{|c|}{ w/o Errors } & 1.15 & 10.12 & 74.50 & 3.33 & 22.17 \\
\hline & \multirow{3}{*}{$\begin{array}{c}\mathrm{w} / \\
\text { Intrinsic } \\
\text { Errors }\end{array}$} & Average & 1.58 & 12.87 & 64.59 & 4.24 & 31.17 \\
\hline & & Minimum & 1.23 & 10.66 & 61.17 & 2.59 & 22.34 \\
\hline & & Maximum & 1.75 & 13.97 & 71.17 & 6.49 & 34.75 \\
\hline
\end{tabular}

\section{A.4 Impacts of Systematic Errors}

Table A-7a Impacts of Systematic Errors on Travel Time Estimation Performance for Simulated Uncongested Conditions without Data Filtering

\begin{tabular}{|l|c|c|c|c|c|c|}
\hline \multirow{2}{*}{ Method } & Cases & $\begin{array}{c}\text { MAE } \\
\text { (Min.) }\end{array}$ & $\begin{array}{c}\text { MAPE } \\
\mathbf{( \% )}\end{array}$ & $\begin{array}{c}\text { Reliability } \\
\mathbf{( \% )}\end{array}$ & \% Early & \% Late \\
\hline $\begin{array}{l}\text { Point-to-Point } \\
\text { Method }\end{array}$ & w/o Errors & 0.12 & 1.92 & 100 & 0 & 0 \\
\cline { 2 - 7 } & Case 1 & 0.17 & 2.67 & 100 & 0 & 0 \\
\cline { 2 - 7 } & Case 2 & 0.22 & 3.51 & 100 & 0 & 0 \\
\cline { 2 - 7 } & Case 3 & 0.26 & 4.07 & 100 & 0 & 0 \\
\cline { 2 - 7 } & Case 4 & 0.23 & 3.65 & 100 & 0 & 0 \\
\cline { 2 - 7 } & Case 5 & 0.32 & 5.09 & 100 & 0 & 0 \\
\cline { 2 - 7 } & Case 6 & 0.40 & 6.29 & 100 & 0 & 0 \\
\cline { 2 - 7 } & Case 7 & 0.09 & 1.43 & 100 & 0 & 0 \\
\cline { 2 - 7 } & Case 8 & 0.08 & 1.29 & 100 & 0 & 0 \\
\cline { 2 - 7 } & Case 9 & 0.10 & 1.58 & 99.73 & 0.27 & 0 \\
\hline
\end{tabular}




\begin{tabular}{|c|c|c|c|c|c|c|}
\hline Method & Cases & $\begin{array}{l}\text { MAE } \\
\text { (Min.) }\end{array}$ & $\begin{array}{c}\text { MAPE } \\
(\%)\end{array}$ & $\begin{array}{c}\text { Reliability } \\
\text { (\%) }\end{array}$ & \% Early & $\%$ Late \\
\hline & Case 10 & 0.08 & 1.26 & 100 & 0 & 0 \\
\hline & Case 11 & 0.11 & 1.75 & 99.53 & 0.47 & 0 \\
\hline & Case 12 & 0.24 & 3.85 & 87.54 & 12.46 & 0 \\
\hline \multirow{13}{*}{$\begin{array}{l}\text { Mid-Point } \\
\text { Method }\end{array}$} & w/o Errors & 0.08 & 1.31 & 100 & 0 & 0 \\
\hline & Case 1 & 0.12 & 1.91 & 100 & 0 & 0 \\
\hline & Case 2 & 0.15 & 2.30 & 100 & 0 & 0 \\
\hline & Case 3 & 0.21 & 3.24 & 100 & 0 & 0 \\
\hline & Case 4 & 0.18 & 2.88 & 100 & 0 & 0 \\
\hline & Case 5 & 0.28 & 4.51 & 100 & 0 & 0 \\
\hline & Case 6 & 0.37 & 5.85 & 100 & 0 & 0 \\
\hline & Case 7 & 0.08 & 1.33 & 100 & 0 & 0 \\
\hline & Case 8 & 0.10 & 1.60 & 99.82 & 0.18 & 0 \\
\hline & Case 9 & 0.16 & 2.54 & 93.85 & 6.15 & 0 \\
\hline & Case 10 & 0.10 & 1.52 & 99.91 & 0.09 & 0 \\
\hline & Case 11 & 0.19 & 3.07 & 91.19 & 8.81 & 0 \\
\hline & Case 12 & 0.35 & 5.57 & 83.58 & 16.42 & 0 \\
\hline \multirow{13}{*}{ Hybrid Model 1} & w/o Errors & 0.08 & 1.31 & 100 & 0 & 0 \\
\hline & Case 1 & 0.12 & 1.91 & 100 & 0 & 0 \\
\hline & Case 2 & 0.15 & 2.30 & 100 & 0 & 0 \\
\hline & Case 3 & 0.21 & 3.24 & 100 & 0 & 0 \\
\hline & Case 4 & 0.18 & 2.88 & 100 & 0 & 0 \\
\hline & Case 5 & 0.28 & 4.51 & 100 & 0 & 0 \\
\hline & Case 6 & 0.37 & 5.85 & 100 & 0 & 0 \\
\hline & Case 7 & 0.08 & 1.27 & 100 & 0 & 0 \\
\hline & Case 8 & 0.09 & 1.45 & 99.95 & 0.05 & 0 \\
\hline & Case 9 & 0.15 & 2.47 & 94.89 & 5.11 & 0 \\
\hline & Case 10 & 0.10 & 1.52 & 99.89 & 0.11 & 0 \\
\hline & Case 11 & 0.19 & 3.04 & 91.19 & 8.81 & 0 \\
\hline & Case 12 & 0.33 & 5.30 & 83.58 & 16.42 & 0 \\
\hline \multirow[t]{8}{*}{ Hybrid Model 2} & w/o Errors & 0.08 & 1.31 & 100 & 0 & 0 \\
\hline & Case 1 & 0.12 & 1.91 & 100 & 0 & 0 \\
\hline & Case 2 & 0.15 & 2.30 & 100 & 0 & 0 \\
\hline & Case 3 & 0.21 & 3.24 & 100 & 0 & 0 \\
\hline & Case 4 & 0.18 & 2.88 & 100 & 0 & 0 \\
\hline & Case 5 & 0.28 & 4.51 & 100 & 0 & 0 \\
\hline & Case 6 & 0.37 & 5.85 & 100 & 0 & 0 \\
\hline & Case 7 & 0.21 & 3.40 & 86.59 & 13.41 & 0 \\
\hline
\end{tabular}




\begin{tabular}{|l|c|c|c|c|c|c|}
\hline Method & Cases & $\begin{array}{c}\text { MAE } \\
\text { (Min.) }\end{array}$ & $\begin{array}{c}\text { MAPE } \\
\mathbf{( \% )}\end{array}$ & $\begin{array}{c}\text { Reliability } \\
\mathbf{( \% )}\end{array}$ & \% Early & \% Late \\
\hline \multirow{6}{*}{ Case 8 } & 0.27 & 4.30 & 86.37 & 13.63 & 0 \\
\cline { 2 - 7 } & Case 9 & 0.38 & 5.96 & 87.06 & 12.95 & 0 \\
\cline { 2 - 7 } & Case 10 & 0.10 & 1.67 & 99.34 & 0.66 & 0 \\
\cline { 2 - 7 } & Case 11 & 0.32 & 5.13 & 90.77 & 9.23 & 0 \\
\cline { 2 - 7 } & Case 12 & 0.71 & 11.29 & 83.58 & 16.42 & 0 \\
\hline
\end{tabular}

Note: the definition for each case is explained in Table 5-4.

Table A-7b Impacts of Systematic Errors on Travel Time Estimation Performance for Simulated Uncongested Conditions with Data Filtering

\begin{tabular}{|c|c|c|c|c|c|c|}
\hline Method & Cases & $\begin{array}{l}\text { MAE } \\
\text { (Min.) }\end{array}$ & $\begin{array}{c}\text { MAPE } \\
(\%)\end{array}$ & $\begin{array}{c}\text { Reliability } \\
(\%)\end{array}$ & \% Early & \% Late \\
\hline \multirow{13}{*}{$\begin{array}{l}\text { Point-to-Point } \\
\text { Method }\end{array}$} & w/o Errors & 0.12 & 1.92 & 100 & 0 & 0 \\
\hline & Case 1 & 0.16 & 2.47 & 100 & 0 & 0 \\
\hline & Case 2 & 0.17 & 2.63 & 100 & 0 & 0 \\
\hline & Case 3 & 0.21 & 3.27 & 100 & 0 & 0 \\
\hline & Case 4 & 0.16 & 2.53 & 100 & 0 & 0 \\
\hline & Case 5 & 0.21 & 3.28 & 100 & 0 & 0 \\
\hline & Case 6 & 0.34 & 5.35 & 100 & 0 & 0 \\
\hline & Case 7 & 0.09 & 1.43 & 100 & 0 & 0 \\
\hline & Case 8 & 0.08 & 1.29 & 100 & 0 & 0 \\
\hline & Case 9 & 0.10 & 1.58 & 99.73 & 0.27 & 0 \\
\hline & Case 10 & 0.08 & 1.26 & 100 & 0 & 0 \\
\hline & Case 11 & 0.11 & 1.76 & 99.43 & 0.57 & 0 \\
\hline & Case 12 & 0.24 & 3.85 & 87.54 & 12.46 & 0 \\
\hline \multirow{10}{*}{$\begin{array}{l}\text { Mid-Point } \\
\text { Method }\end{array}$} & w/o Errors & 0.08 & 1.31 & 100 & 0 & 0 \\
\hline & Case 1 & 0.11 & 1.72 & 100 & 0 & 0 \\
\hline & Case 2 & 0.11 & 1.77 & 100 & 0 & 0 \\
\hline & Case 3 & 0.17 & 2.69 & 100 & 0 & 0 \\
\hline & Case 4 & 0.11 & 1.80 & 100 & 0 & 0 \\
\hline & Case 5 & 0.17 & 2.69 & 100 & 0 & 0 \\
\hline & Case 6 & 0.33 & 5.15 & 100 & 0 & 0 \\
\hline & Case 7 & 0.08 & 1.33 & 100 & 0 & 0 \\
\hline & Case 8 & 0.10 & 1.60 & 99.65 & 0.36 & 0 \\
\hline & Case 9 & 0.16 & 2.55 & 94.00 & 6.01 & 0 \\
\hline
\end{tabular}




\begin{tabular}{|c|c|c|c|c|c|c|}
\hline Method & Cases & $\begin{array}{l}\text { MAE } \\
\text { (Min.) }\end{array}$ & $\begin{array}{c}\text { MAPE } \\
(\%)\end{array}$ & $\begin{array}{c}\text { Reliability } \\
\text { (\%) }\end{array}$ & \% Early & $\%$ Late \\
\hline & Case 10 & 0.10 & 1.53 & 99.94 & 0.06 & 0 \\
\hline & Case 11 & 0.19 & 3.08 & 91.19 & 8.81 & 0 \\
\hline & Case 12 & 0.35 & 5.57 & 83.58 & 16.42 & 0 \\
\hline \multirow{13}{*}{ Hybrid Model 1} & w/o Errors & 0.08 & 1.31 & 100 & 0 & 0 \\
\hline & Case 1 & 0.11 & 1.72 & 100 & 0 & 0 \\
\hline & Case 2 & 0.11 & 1.77 & 100 & 0 & 0 \\
\hline & Case 3 & 0.17 & 2.69 & 100 & 0 & 0 \\
\hline & Case 4 & 0.11 & 1.80 & 100 & 0 & 0 \\
\hline & Case 5 & 0.17 & 2.69 & 100 & 0 & 0 \\
\hline & Case 6 & 0.33 & 5.15 & 100 & 0 & 0 \\
\hline & Case 7 & 0.08 & 1.27 & 100 & 0 & 0 \\
\hline & Case 8 & 0.08 & 1.32 & 100 & 0 & 0 \\
\hline & Case 9 & 0.13 & 2.11 & 98.66 & 1.34 & 0 \\
\hline & Case 10 & 0.10 & 1.53 & 99.89 & 0.11 & 0 \\
\hline & Case 11 & 0.19 & 3.04 & 91.19 & 8.81 & 0 \\
\hline & Case 12 & 0.31 & 4.91 & 83.58 & 16.42 & 0 \\
\hline \multirow{13}{*}{ Hybrid Model 2} & w/o Errors & 0.08 & 1.31 & 100 & 0 & 0 \\
\hline & Case 1 & 0.11 & 1.72 & 100 & 0 & 0 \\
\hline & Case 2 & 0.11 & 1.77 & 100 & 0 & 0 \\
\hline & Case 3 & 0.17 & 2.68 & 100 & 0 & 0 \\
\hline & Case 4 & 0.11 & 1.80 & 100 & 0 & 0 \\
\hline & Case 5 & 0.17 & 2.69 & 100 & 0 & 0 \\
\hline & Case 6 & 0.33 & 5.15 & 100 & 0 & 0 \\
\hline & Case 7 & 0.21 & 3.40 & 86.59 & 13.41 & 0 \\
\hline & Case 8 & 0.27 & 4.30 & 86.37 & 13.63 & 0 \\
\hline & Case 9 & 0.38 & 5.95 & 87.06 & 12.95 & 0 \\
\hline & Case 10 & 0.10 & 1.67 & 99.34 & 0.66 & 0 \\
\hline & Case 11 & 0.32 & 5.13 & 90.77 & 9.23 & 0 \\
\hline & Case 12 & 0.71 & 11.29 & 83.58 & 16.42 & 0 \\
\hline
\end{tabular}


Table A-8a Impacts of Systematic Errors on Travel Time Estimation Performance for Simulated Incident Conditions without Data Filtering

\begin{tabular}{|c|c|c|c|c|c|c|}
\hline Method & Cases & $\begin{array}{l}\text { MAE } \\
\text { (Min.) }\end{array}$ & $\begin{array}{c}\text { MAPE } \\
(\%)\end{array}$ & $\begin{array}{c}\text { Reliability } \\
\text { (\%) }\end{array}$ & $\%$ Early & \% Late \\
\hline \multirow{13}{*}{$\begin{array}{l}\text { Point-to-Point } \\
\text { Method }\end{array}$} & w/o Errors & 2.07 & 16.28 & 57.32 & 1.78 & 40.90 \\
\hline & Case 1 & 2.09 & 16.47 & 57.50 & 1.61 & 40.90 \\
\hline & Case 2 & 2.15 & 16.97 & 57.50 & 1.61 & 40.90 \\
\hline & Case 3 & 2.25 & 17.98 & 57.27 & 1.61 & 41.13 \\
\hline & Case 4 & 2.52 & 19.66 & 55.20 & 0.23 & 44.57 \\
\hline & Case 5 & 2.76 & 21.81 & 53.99 & 0 & 46.01 \\
\hline & Case 6 & 2.92 & 23.40 & 52.67 & 0 & 47.33 \\
\hline & Case 7 & 2.04 & 16.09 & 58.07 & 3.10 & 38.83 \\
\hline & Case 8 & 2.02 & 15.92 & 60.14 & 3.50 & 36.36 \\
\hline & Case 9 & 1.93 & 15.70 & 56.40 & 8.73 & 34.87 \\
\hline & Case 10 & 1.84 & 14.71 & 58.36 & 6.78 & 34.87 \\
\hline & Case 11 & 1.83 & 15.20 & 61.69 & 9.88 & 28.43 \\
\hline & Case 12 & 2.30 & 20.40 & 46.76 & 35.38 & 17.86 \\
\hline \multirow{13}{*}{$\begin{array}{l}\text { Mid-Point } \\
\text { Method }\end{array}$} & w/o Errors & 1.86 & 14.78 & 59.05 & 4.19 & 36.76 \\
\hline & Case 1 & 1.89 & 14.98 & 59.51 & 3.10 & 37.39 \\
\hline & Case 2 & 1.91 & 15.11 & 59.10 & 2.64 & 38.25 \\
\hline & Case 3 & 2.04 & 16.28 & 59.45 & 1.61 & 38.94 \\
\hline & Case 4 & 2.49 & 19.35 & 53.65 & 0.00 & 46.35 \\
\hline & Case 5 & 2.81 & 22.15 & 52.56 & 0.00 & 47.44 \\
\hline & Case 6 & 3.01 & 24.03 & 51.23 & 0.00 & 48.77 \\
\hline & Case 7 & 1.83 & 14.67 & 60.77 & 4.25 & 34.98 \\
\hline & Case 8 & 1.82 & 14.61 & 60.20 & 5.05 & 34.75 \\
\hline & Case 9 & 1.80 & 15.31 & 60.60 & 9.82 & 29.58 \\
\hline & Case 10 & 1.63 & 13.55 & 67.20 & 6.55 & 26.25 \\
\hline & Case 11 & 1.82 & 16.10 & 54.80 & 22.46 & 22.75 \\
\hline & Case 12 & 3.46 & 29.78 & 33.95 & 48.48 & 17.58 \\
\hline \multirow[t]{5}{*}{ Hybrid Model 1} & w/o Errors & 1.11 & 10.24 & 70.59 & 10.34 & 19.07 \\
\hline & Case 1 & 1.08 & 9.90 & 73.75 & 6.72 & 19.53 \\
\hline & Case 2 & 1.09 & 9.95 & 73.75 & 6.72 & 19.53 \\
\hline & Case 3 & 1.20 & 10.53 & 76.62 & 2.41 & 20.96 \\
\hline & Case 4 & 1.15 & 10.19 & 76.68 & 2.13 & 21.19 \\
\hline
\end{tabular}




\begin{tabular}{|c|c|c|c|c|c|c|}
\hline Method & Cases & $\begin{array}{l}\text { MAE } \\
\text { (Min.) }\end{array}$ & $\begin{array}{c}\text { MAPE } \\
(\%)\end{array}$ & $\begin{array}{c}\text { Reliability } \\
\text { (\%) }\end{array}$ & \% Early & \% Late \\
\hline & Case 5 & 1.21 & 10.73 & 76.79 & 1.78 & 21.42 \\
\hline & Case 6 & 1.36 & 12.09 & 76.28 & 0.92 & 22.80 \\
\hline & Case 7 & 1.45 & 12.62 & 69.84 & 13.50 & 16.66 \\
\hline & Case 8 & 1.46 & 13.01 & 59.51 & 23.84 & 16.66 \\
\hline & Case 9 & 1.01 & 10.03 & 69.73 & 15.57 & 14.70 \\
\hline & Case 10 & 1.20 & 11.23 & 66.17 & 18.09 & 15.74 \\
\hline & Case 11 & 1.46 & 13.01 & 59.16 & 24.35 & 16.48 \\
\hline & Case 12 & 1.84 & 16.97 & 54.68 & 30.67 & 14.65 \\
\hline \multirow{13}{*}{ Hybrid Model 2} & w/o Errors & 1.15 & 10.12 & 74.50 & 3.33 & 22.17 \\
\hline & Case 1 & 1.16 & 10.17 & 74.38 & 2.99 & 22.63 \\
\hline & Case 2 & 1.16 & 10.21 & 74.84 & 2.53 & 22.63 \\
\hline & Case 3 & 1.25 & 11.05 & 74.38 & 1.49 & 24.12 \\
\hline & Case 4 & 1.99 & 15.88 & 60.02 & 0.23 & 39.75 \\
\hline & Case 5 & 2.37 & 19.00 & 54.62 & 0.23 & 45.15 \\
\hline & Case 6 & 2.54 & 20.62 & 53.88 & 0.23 & 45.89 \\
\hline & Case 7 & 1.50 & 13.11 & 64.39 & 15.11 & 20.51 \\
\hline & Case 8 & 1.25 & 10.95 & 71.22 & 7.64 & 21.14 \\
\hline & Case 9 & 1.10 & 10.47 & 75.07 & 8.73 & 16.20 \\
\hline & Case 10 & 1.58 & 13.26 & 61.86 & 15.91 & 22.23 \\
\hline & Case 11 & 1.69 & 14.70 & 52.38 & 22.92 & 24.70 \\
\hline & Case 12 & 5.62 & 42.88 & 41.53 & 39.06 & 19.41 \\
\hline
\end{tabular}

Table A-8b Impacts of Systematic Errors on Travel Time Estimation Performance for Simulated Incident Conditions with Data Filtering

\begin{tabular}{|l|c|c|c|c|c|c|}
\hline \multirow{2}{*}{ Method } & Cases & $\begin{array}{c}\text { MAE } \\
\text { (Min.) }\end{array}$ & $\begin{array}{c}\text { MAPE } \\
\mathbf{( \% )}\end{array}$ & $\begin{array}{c}\text { Reliability } \\
\mathbf{( \% )}\end{array}$ & \% Early & \% Late \\
\hline $\begin{array}{l}\text { Point-to-Point } \\
\text { Method }\end{array}$ & w/o Errors & 2.07 & 16.28 & 57.32 & 1.78 & 40.90 \\
\cline { 2 - 7 } & Case 1 & 2.08 & 16.33 & 57.38 & 1.72 & 40.90 \\
\cline { 2 - 7 } & Case 2 & 2.08 & 16.35 & 57.38 & 1.72 & 40.90 \\
\cline { 2 - 7 } & Case 3 & 2.18 & 17.18 & 57.27 & 1.61 & 41.13 \\
\cline { 2 - 7 } & Case 4 & 2.08 & 16.47 & 58.36 & 2.13 & 39.52 \\
\cline { 2 - 7 } & Case 5 & 2.30 & 18.21 & 58.64 & 0.23 & 41.13 \\
\cline { 2 - 7 } & Case 6 & 2.79 & 22.03 & 52.84 & 0.00 & 47.16 \\
\hline
\end{tabular}




\begin{tabular}{|c|c|c|c|c|c|c|}
\hline Method & Cases & $\begin{array}{c}\text { MAE } \\
\text { (Min.) }\end{array}$ & $\begin{array}{c}\text { MAPE } \\
(\%)\end{array}$ & $\begin{array}{c}\text { Reliability } \\
\text { (\%) }\end{array}$ & \% Early & \% Late \\
\hline & Case 7 & 2.04 & 16.08 & 58.76 & 3.10 & 38.14 \\
\hline & Case 8 & 2.01 & 15.91 & 60.14 & 3.50 & 36.36 \\
\hline & Case 9 & 2.03 & 15.97 & 60.94 & 2.87 & 36.19 \\
\hline & Case 10 & 1.99 & 15.65 & 57.90 & 4.37 & 37.74 \\
\hline & Case 11 & 1.97 & 15.71 & 57.84 & 6.38 & 35.78 \\
\hline & Case 12 & 2.11 & 17.76 & 56.98 & 13.79 & 29.24 \\
\hline \multirow{13}{*}{$\begin{array}{l}\text { Mid-Point } \\
\text { Method }\end{array}$} & w/o Errors & 1.86 & 14.78 & 59.05 & 4.19 & 36.76 \\
\hline & Case 1 & 1.87 & 14.79 & 58.99 & 3.62 & 37.39 \\
\hline & Case 2 & 1.87 & 14.80 & 58.99 & 3.62 & 37.39 \\
\hline & Case 3 & 2.00 & 15.83 & 59.33 & 1.72 & 38.94 \\
\hline & Case 4 & 1.89 & 15.20 & 60.37 & 2.70 & 36.93 \\
\hline & Case 5 & 2.18 & 17.33 & 58.07 & 0.23 & 41.70 \\
\hline & Case 6 & 2.90 & 22.95 & 52.67 & 0.00 & 47.33 \\
\hline & Case 7 & 1.84 & 14.68 & 60.83 & 4.42 & 34.75 \\
\hline & Case 8 & 1.82 & 14.62 & 60.20 & 5.05 & 34.75 \\
\hline & Case 9 & 1.84 & 14.84 & 59.74 & 5.51 & 34.75 \\
\hline & Case 10 & 1.81 & 14.59 & 61.34 & 5.05 & 33.60 \\
\hline & Case 11 & 1.89 & 15.81 & 61.34 & 8.39 & 30.27 \\
\hline & Case 12 & 2.77 & 22.59 & 48.02 & 21.42 & 30.56 \\
\hline \multirow{13}{*}{ Hybrid Model 1} & w/o Errors & 1.11 & 10.24 & 70.59 & 10.34 & 19.07 \\
\hline & Case 1 & 1.14 & 10.47 & 71.11 & 9.36 & 19.53 \\
\hline & Case 2 & 1.14 & 10.49 & 71.11 & 9.36 & 19.53 \\
\hline & Case 3 & 1.09 & 9.80 & 75.65 & 4.02 & 20.33 \\
\hline & Case 4 & 1.66 & 13.64 & 69.39 & 3.04 & 27.57 \\
\hline & Case 5 & 1.73 & 14.22 & 66.69 & 2.70 & 30.61 \\
\hline & Case 6 & 1.64 & 13.84 & 67.09 & 2.64 & 30.27 \\
\hline & Case 7 & 1.44 & 12.57 & 69.84 & 13.50 & 16.66 \\
\hline & Case 8 & 1.46 & 12.93 & 60.77 & 22.57 & 16.66 \\
\hline & Case 9 & 1.05 & 10.35 & 66.97 & 19.30 & 13.73 \\
\hline & Case 10 & 1.61 & 13.69 & 66.40 & 8.90 & 24.70 \\
\hline & Case 11 & 1.52 & 12.94 & 68.87 & 5.74 & 25.39 \\
\hline & Case 12 & 1.46 & 13.05 & 64.62 & 13.79 & 21.60 \\
\hline
\end{tabular}




\begin{tabular}{|l|c|c|c|c|c|c|}
\hline Method & Cases & $\begin{array}{c}\text { MAE } \\
\mathbf{( M i n} .)\end{array}$ & $\begin{array}{c}\text { MAPE } \\
\mathbf{( \% )}\end{array}$ & $\begin{array}{c}\text { Reliability } \\
\mathbf{( \% )}\end{array}$ & \% Early & \% Late \\
\hline \multirow{5}{*}{ Ho Errors } & 1.15 & 10.12 & 74.50 & 3.33 & 22.17 \\
\cline { 2 - 7 } & Case 1 & 1.15 & 10.09 & 74.38 & 2.99 & 22.63 \\
\cline { 2 - 7 } & Case 2 & 1.15 & 10.09 & 74.38 & 2.99 & 22.63 \\
\cline { 2 - 7 } & Case 3 & 1.22 & 10.71 & 74.84 & 1.61 & 23.55 \\
\cline { 2 - 7 } & Case 4 & 1.28 & 11.17 & 70.76 & 5.28 & 23.95 \\
\cline { 2 - 7 } & Case 5 & 1.53 & 13.05 & 67.43 & 2.35 & 30.21 \\
\cline { 2 - 7 } & Case 6 & 2.50 & 20.13 & 53.88 & 0.23 & 45.89 \\
\cline { 2 - 7 } & Case 7 & 1.50 & 13.13 & 64.45 & 15.28 & 20.28 \\
\cline { 2 - 7 } & Case 8 & 1.25 & 10.93 & 71.22 & 7.64 & 21.14 \\
\cline { 2 - 7 } & Case 9 & 1.08 & 9.78 & 75.53 & 4.65 & 19.82 \\
\cline { 2 - 7 } & Case 10 & 1.22 & 10.78 & 70.42 & 9.02 & 20.56 \\
\cline { 2 - 7 } & Case 11 & 1.50 & 13.05 & 64.96 & 12.23 & 22.80 \\
\cline { 2 - 7 } & Case 12 & 3.46 & 25.72 & 55.43 & 15.16 & 29.41 \\
\hline
\end{tabular}

Table A-9 Impacts of Systematic Errors in Low Speed Measurements on Travel Time Estimation Performance for Simulated Incident Conditions

\begin{tabular}{|c|c|c|c|c|c|c|}
\hline Method & Cases & $\begin{array}{c}\text { MAE } \\
\text { (Min.) }\end{array}$ & $\begin{array}{c}\text { MAPE } \\
(\%)\end{array}$ & $\begin{array}{c}\text { Reliability } \\
(\%)\end{array}$ & $\begin{array}{c}\% \\
\text { Early }\end{array}$ & $\begin{array}{c}\% \\
\text { Late }\end{array}$ \\
\hline \multirow{5}{*}{$\begin{array}{l}\text { Point-to-Point } \\
\text { Method }\end{array}$} & w/o Errors & 2.07 & 16.28 & 57.32 & 1.78 & 40.90 \\
\hline & 20\% Increase in Low Speed & 2.24 & 17.46 & 55.08 & 0.34 & 44.57 \\
\hline & 40\% Increase in Low Speed & 2.42 & 18.86 & 54.62 & 0.11 & 45.26 \\
\hline & 20\% Decrease in Low Speed & 1.92 & 15.38 & 57.73 & 6.09 & 36.19 \\
\hline & $40 \%$ Decrease in Low Speed & 1.76 & 14.50 & 63.47 & 7.58 & 28.95 \\
\hline \multirow{5}{*}{$\begin{array}{l}\text { Mid-Point } \\
\text { Method }\end{array}$} & w/o Errors & 1.86 & 14.78 & 59.05 & 4.19 & 36.76 \\
\hline & $20 \%$ Increase in Low Speed & 2.08 & 16.31 & 60.65 & 1.21 & 38.14 \\
\hline & 40\% Increase in Low Speed & 2.28 & 17.76 & 54.22 & 0.98 & 44.80 \\
\hline & 20\% Decrease in Low Speed & 1.68 & 13.75 & 62.67 & 7.06 & 30.27 \\
\hline & 40\% Decrease in Low Speed & 1.59 & 13.56 & 66.28 & 9.82 & 23.89 \\
\hline \multirow[t]{5}{*}{ Hybrid Model 1} & \begin{tabular}{|c|} 
w/o Errors \\
\end{tabular} & 1.11 & 10.24 & 70.59 & 10.34 & 19.07 \\
\hline & $20 \%$ Increase in Low Speed & 1.18 & 10.68 & 67.89 & 11.83 & 20.28 \\
\hline & 40\% Increase in Low Speed & 1.23 & 10.87 & 68.06 & 10.68 & 21.25 \\
\hline & 20\% Decrease in Low Speed & 1.12 & 10.31 & 68.35 & 12.58 & 19.07 \\
\hline & $40 \%$ Decrease in Low Speed & 1.25 & 11.24 & 67.78 & 10.97 & 21.25 \\
\hline
\end{tabular}




\begin{tabular}{|c|c|c|c|c|c|c|}
\hline Method & Cases & $\begin{array}{c}\text { MAE } \\
\text { (Min.) }\end{array}$ & $\begin{array}{c}\text { MAPE } \\
\mathbf{( \% )}\end{array}$ & $\begin{array}{c}\text { Reliability } \\
\mathbf{( \% )}\end{array}$ & $\begin{array}{c}\text { \% } \\
\text { Early }\end{array}$ & $\begin{array}{c}\text { \% } \\
\text { Late }\end{array}$ \\
\hline \multirow{5}{*}{ 5\% Increase in Volume } & 1.10 & 9.91 & 72.95 & 7.98 & 19.07 \\
\cline { 2 - 7 } & 10\% Increase in Volume & 1.17 & 10.91 & 64.33 & 16.54 & 19.13 \\
\cline { 2 - 7 } & 5\% Decrease in Volume & 1.17 & 11.10 & 67.55 & 14.30 & 18.15 \\
\cline { 2 - 7 } & 10\% Decrease in Volume & 1.08 & 10.06 & 71.74 & 9.59 & 18.67 \\
\hline \multirow{5}{*}{ Hybrid Model Errors } & 1.15 & 10.12 & 74.50 & 3.33 & 22.17 \\
\cline { 2 - 7 } & $20 \%$ Increase in Low Speed & 1.37 & 11.52 & 71.97 & 1.21 & 26.82 \\
\cline { 2 - 7 } & 40\% Increase in Low Speed & 1.66 & 13.57 & 68.18 & 0.98 & 30.84 \\
\cline { 2 - 7 } & 20\% Decrease in Low Speed & 1.46 & 12.33 & 61.69 & 16.94 & 21.37 \\
\cline { 2 - 7 } & 40\% Decrease in Low Speed & 1.88 & 15.19 & 57.15 & 16.83 & 26.02 \\
\cline { 2 - 7 } & 5\% Increase in Volume & 1.15 & 10.15 & 74.50 & 3.33 & 22.17 \\
\cline { 2 - 7 } & 10\% Increase in Volume & 1.16 & 10.18 & 74.55 & 3.50 & 21.94 \\
\cline { 2 - 7 } & 5\% Decrease in Volume & 1.15 & 10.12 & 74.50 & 3.33 & 22.17 \\
\cline { 2 - 7 } & 10\% Decrease in Volume & 1.15 & 10.12 & 74.50 & 3.33 & 22.17 \\
\hline
\end{tabular}

\section{A.5 Impacts of Incidental and Structural Failures}

Table A-10 Impacts of Incidental and Structural Failures on Travel Time Estimation Performance for Simulated Uncongested Conditions

\begin{tabular}{|l|c|c|c|c|c|c|}
\hline \multirow{2}{*}{ Method } & Cases & $\begin{array}{c}\text { MAE } \\
\mathbf{( M i n . )}\end{array}$ & $\begin{array}{c}\text { MAPE } \\
\mathbf{( \% )}\end{array}$ & $\begin{array}{c}\text { Reliability } \\
\mathbf{( \% )}\end{array}$ & \% Early & \% Late \\
\hline \multirow{2}{*}{$\begin{array}{l}\text { Point to Point } \\
\text { Method }\end{array}$} & w/o Errors & 0.12 & 1.92 & 100 & 0 & 0 \\
\cline { 2 - 7 } & $\begin{array}{c}\text { w/ Incidental and } \\
\text { Structural Errors }\end{array}$ & 0.14 & 2.18 & 100 & 0 & 0 \\
\hline \multirow{2}{*}{$\begin{array}{l}\text { Mid-Point } \\
\text { Method }\end{array}$} & $\begin{array}{c}\text { w/ Incidental and } \\
\text { Structural Errors }\end{array}$ & 0.10 & 1.53 & 100 & 0 & 0 \\
\hline \multirow{2}{*}{ Hybrid Model 1 } & w/o Errors & 0.08 & 1.31 & 100 & 0 & 0 \\
\cline { 2 - 7 } & $\begin{array}{c}\text { w/ Incidental and } \\
\text { Structural Errors }\end{array}$ & 0.10 & 1.53 & 100 & 0 & 0 \\
\hline \multirow{2}{*}{ Hybrid Model 2 } & w/o Errors & 0.08 & 1.31 & 100 & 0 & 0 \\
\cline { 2 - 7 } & $\begin{array}{c}\text { w/ Incidental and } \\
\text { Structural Errors }\end{array}$ & 0.10 & 1.54 & 100 & 0 & 0 \\
\hline
\end{tabular}


Table A-11 Impacts of Incidental and Structural Failures on Travel Time Estimation Performance for Simulated Incident Conditions

\begin{tabular}{|l|c|c|c|c|c|c|}
\hline \multirow{2}{*}{ Method } & Cases & $\begin{array}{c}\text { MAE } \\
\text { (Min.) }\end{array}$ & $\begin{array}{c}\text { MAPE } \\
\mathbf{( \% )}\end{array}$ & $\begin{array}{c}\text { Reliability } \\
\mathbf{( \% )}\end{array}$ & \% Early & \% Late \\
\hline $\begin{array}{l}\text { Point to Point } \\
\text { Method }\end{array}$ & w/o Errors & 2.07 & 16.28 & 57.32 & 1.78 & 40.90 \\
\cline { 2 - 7 } & $\begin{array}{l}\text { w/ Incidental and } \\
\text { Structural Errors }\end{array}$ & 2.04 & 16.21 & 55.66 & 6.61 & 37.74 \\
\hline \multirow{2}{*}{$\begin{array}{l}\text { Mid-Point } \\
\text { Method }\end{array}$} & w/o Errors & 1.86 & 14.78 & 59.05 & 4.19 & 36.76 \\
\cline { 2 - 7 } & $\begin{array}{l}\text { w/ Incidental and } \\
\text { Structural Errors }\end{array}$ & 2.06 & 17.00 & 58.07 & 10.57 & 31.36 \\
\hline \multirow{2}{*}{ Hybrid Model 1 } & w/o Errors & 1.11 & 10.24 & 70.59 & 10.34 & 19.07 \\
\cline { 2 - 7 } & $\begin{array}{l}\text { w/ Incidental and } \\
\text { Structural Errors }\end{array}$ & 1.80 & 16.36 & 58.13 & 25.16 & 16.72 \\
\hline \multirow{2}{*}{ Hybrid Model 2 } & w/o Errors & 1.15 & 10.12 & 74.50 & 3.33 & 22.17 \\
\cline { 2 - 7 } & $\begin{array}{l}\text { w/ Incidental and } \\
\text { Structural Errors }\end{array}$ & 1.95 & 17.58 & 62.44 & 15.45 & 22.11 \\
\hline
\end{tabular}

\section{A.6 Impacts of Detector Spacing}

Table A-12 Impacts of Detector Spacing on Travel Time Estimation Performance for Simulated Uncongested Conditions

\begin{tabular}{|l|c|c|c|c|c|c|}
\hline \multirow{2}{*}{ Method } & $\begin{array}{c}\text { Approximate } \\
\text { Detector Spacing } \\
\text { (Miles) }\end{array}$ & $\begin{array}{c}\text { MAE } \\
\text { (Min.) }\end{array}$ & $\begin{array}{c}\text { MAPE } \\
\mathbf{( \% )}\end{array}$ & $\begin{array}{c}\text { Reliability } \\
\mathbf{( \% )}\end{array}$ & \% Early & \% Late \\
\hline \multirow{2}{*}{$\begin{array}{l}\text { Point-to-Point } \\
\text { Method }\end{array}$} & 0.3 & 0.12 & 1.92 & 100 & 0 & 0 \\
\cline { 2 - 7 } & 0.6 & 0.14 & 2.29 & 100 & 0 & 0 \\
\hline \multirow{3}{*}{$\begin{array}{l}\text { Mid-Point } \\
\text { Method }\end{array}$} & 1.2 & 0.08 & 1.29 & 99.94 & 0.06 & 0 \\
\cline { 2 - 7 } & 0.3 & 0.08 & 1.31 & 100 & 0 & 0 \\
\cline { 2 - 7 } & 0.6 & 0.10 & 1.57 & 100 & 0 & 0 \\
\hline \multirow{3}{*}{ Hybrid Model 1 } & 1.2 & 0.09 & 1.40 & 99.75 & 0.25 & 0 \\
\cline { 2 - 7 } & 0.3 & 0.08 & 1.31 & 100 & 0 & 0 \\
\cline { 2 - 7 } & 0.6 & 0.10 & 1.57 & 100 & 0 & 0 \\
\cline { 2 - 7 } & 1.2 & 0.09 & 1.40 & 99.75 & 0.25 & 0 \\
\cline { 2 - 7 } & 0.3 & 0.08 & 1.31 & 100 & 0 & 0 \\
\cline { 2 - 7 } & 0.6 & 0.10 & 1.57 & 100 & 0 & 0 \\
\hline
\end{tabular}


Table A-13 Impacts of Detector Spacing on Travel Time Estimation Performance for Simulated Incident Conditions

\begin{tabular}{|l|c|c|c|c|c|c|}
\hline \multirow{2}{*}{ Method } & $\begin{array}{c}\text { Approximate } \\
\text { Detector Spacing } \\
\text { (Miles) }\end{array}$ & $\begin{array}{c}\text { MAE } \\
\text { (Min.) }\end{array}$ & $\begin{array}{c}\text { MAPE } \\
\mathbf{( \% )}\end{array}$ & $\begin{array}{c}\text { Reliability } \\
\mathbf{( \% )}\end{array}$ & \% Early & \% Late \\
\hline \multirow{3}{*}{$\begin{array}{l}\text { Point-to-Point } \\
\text { Method }\end{array}$} & 0.3 & 2.07 & 16.28 & 57.32 & 1.78 & 40.90 \\
\cline { 2 - 7 } & 0.6 & 2.86 & 21.96 & 53.99 & 0.46 & 45.55 \\
\cline { 2 - 7 } Mid-Point & 1.2 & 2.86 & 22.08 & 52.15 & 0.86 & 46.98 \\
\hline \multirow{3}{*}{ Method } & 0.3 & 1.86 & 14.78 & 59.05 & 4.19 & 36.76 \\
\hline \multirow{3}{*}{ Hybrid Model 1 } & 0.6 & 2.85 & 21.85 & 51.52 & 0.92 & 47.56 \\
\cline { 2 - 7 } & 1.2 & 2.77 & 21.30 & 51.81 & 1.26 & 46.93 \\
\cline { 2 - 7 } & 0.3 & 1.11 & 10.24 & 70.59 & 10.34 & 19.07 \\
\hline \multirow{3}{*}{ Hybrid Model 2 } & 0.6 & 1.89 & 15.13 & 63.18 & 1.67 & 35.15 \\
\cline { 2 - 7 } & 1.2 & 1.93 & 15.38 & 56.81 & 4.02 & 39.17 \\
\cline { 2 - 7 } & 0.3 & 1.15 & 10.12 & 74.50 & 3.33 & 22.17 \\
\hline
\end{tabular}

\section{A.7 Impacts of Travel Time Updating Frequency}

Table A-14 Travel Time Estimation Performances with Different Travel Time Updating Frequencies for Simulated Uncongested Conditions

\begin{tabular}{|l|c|c|c|c|c|c|}
\hline \multirow{4}{*}{ Method } & $\begin{array}{c}\text { Updating } \\
\text { Frequency }\end{array}$ & $\begin{array}{c}\text { MAE } \\
\text { (Min.) }\end{array}$ & $\begin{array}{c}\text { MAPE } \\
\mathbf{( \% )}\end{array}$ & $\begin{array}{c}\text { Reliability } \\
\mathbf{( \% )}\end{array}$ & \% Early & \% Late \\
\hline \multirow{4}{*}{\begin{tabular}{l} 
Moint-to-Point \\
\cline { 2 - 7 }
\end{tabular}} & 1-minute & 0.14 & 2.20 & 100 & 0 & 0 \\
\cline { 2 - 7 } & 2-minute & 0.12 & 1.92 & 100 & 0 & 0 \\
\cline { 2 - 7 } & 3-minute & 0.12 & 1.91 & 100 & 0 & 0 \\
\cline { 2 - 7 } & 4-minute & 0.12 & 1.90 & 100 & 0 & 0 \\
\cline { 2 - 7 } & 5-minute & 0.12 & 1.86 & 100 & 0 & 0 \\
\hline \multirow{4}{*}{ Mid-Point } & 1-minute & 0.10 & 1.62 & 100 & 0 & 0 \\
\cline { 2 - 7 } & 2-minute & 0.08 & 1.31 & 100 & 0 & 0 \\
\cline { 2 - 7 } & 3-minute & 0.07 & 1.16 & 100 & 0 & 0 \\
\cline { 2 - 7 } & 4-minute & 0.07 & 1.15 & 100 & 0 & 0 \\
\cline { 2 - 7 } & 5-minute & 0.06 & 1.03 & 100 & 0 & 0 \\
\hline Hybrid Model 1 & 1-minute & 0.10 & 1.62 & 100 & 0 & 0 \\
\cline { 2 - 7 } & 2-minute & 0.08 & 1.31 & 100 & 0 & 0 \\
\hline
\end{tabular}




\begin{tabular}{|l|c|c|c|c|c|c|}
\hline Method & $\begin{array}{c}\text { Updating } \\
\text { Frequency }\end{array}$ & $\begin{array}{c}\text { MAE } \\
\text { (Min.) }\end{array}$ & $\begin{array}{c}\text { MAPE } \\
\mathbf{( \% )}\end{array}$ & $\begin{array}{c}\text { Reliability } \\
\mathbf{( \% )}\end{array}$ & \% Early & \% Late \\
\hline \multirow{5}{*}{ Hybrid Model 2 } & 3-minute & 0.07 & 1.16 & 100 & 0 & 0 \\
\cline { 2 - 7 } & 4-minute & 0.07 & 1.15 & 100 & 0 & 0 \\
\cline { 2 - 7 } & 5-minute & 0.07 & 1.03 & 100 & 0 & 0 \\
\cline { 2 - 7 } & 1-minute & 0.10 & 1.62 & 100 & 0 & 0 \\
\cline { 2 - 7 } & 2-minute & 0.08 & 1.31 & 100 & 0 & 0 \\
\cline { 2 - 7 } & 4-minute & 0.07 & 1.16 & 100 & 0 & 0 \\
\cline { 2 - 7 } & 5-minute & 0.07 & 1.15 & 100 & 0 & 0 \\
\hline
\end{tabular}

Table A-15 Travel Time Estimation Performances with Different Travel Time Updating Frequencies for Simulated Incident Conditions

\begin{tabular}{|c|c|c|c|c|c|c|}
\hline Method & $\begin{array}{l}\text { Updating } \\
\text { Frequency }\end{array}$ & $\begin{array}{l}\text { MAE } \\
\text { (Min.) }\end{array}$ & $\begin{array}{c}\text { MAPE } \\
(\%)\end{array}$ & $\begin{array}{c}\text { Reliability } \\
(\%)\end{array}$ & \% Early & \% Late \\
\hline \multirow{5}{*}{$\begin{array}{l}\text { Point-to-Point } \\
\text { Method }\end{array}$} & 1-minute & 1.99 & 15.63 & 59.27 & 0.80 & 39.93 \\
\hline & 2-minute & 2.07 & 16.28 & 57.32 & 1.78 & 40.90 \\
\hline & 3-minute & 2.04 & 16.05 & 55.83 & 3.83 & 40.35 \\
\hline & 4-minute & 2.29 & 18.19 & 48.17 & 8.67 & 43.17 \\
\hline & 5-minute & 2.25 & 17.58 & 52.85 & 6.75 & 40.41 \\
\hline \multirow{5}{*}{$\begin{array}{l}\text { Mid-Point } \\
\text { Method }\end{array}$} & 1-minute & 1.79 & 14.23 & 60.99 & 1.89 & 37.12 \\
\hline & 2-minute & 1.86 & 14.78 & 59.05 & 4.19 & 36.76 \\
\hline & 3-minute & 1.86 & 14.82 & 57.62 & 6.15 & 36.23 \\
\hline & 4-minute & 2.08 & 16.81 & 47.70 & 14.37 & 37.93 \\
\hline & 5-minute & 2.12 & 16.77 & 54.37 & 7.92 & 37.71 \\
\hline \multirow{5}{*}{ Hybrid Model 1} & 1-minute & 1.10 & 9.85 & 73.38 & 9.07 & 17.56 \\
\hline & 2-minute & 1.11 & 10.24 & 70.59 & 10.34 & 19.07 \\
\hline & 3-minute & 1.15 & 10.54 & 69.04 & 12.46 & 18.49 \\
\hline & 4-minute & 1.54 & 13.77 & 46.77 & 25.31 & 27.92 \\
\hline & 5-minute & 1.27 & 11.29 & 66.33 & 8.80 & 24.87 \\
\hline \multirow{5}{*}{ Hybrid Model 2} & 1-minute & 1.16 & 10.21 & 74.24 & 2.58 & 23.18 \\
\hline & 2-minute & 1.15 & 10.12 & 74.50 & 3.33 & 22.17 \\
\hline & 3-minute & 1.31 & 11.31 & 70.55 & 4.64 & 24.81 \\
\hline & 4-minute & 1.35 & 11.85 & 55.38 & 17.69 & 26.93 \\
\hline & 5-minute & 1.34 & 11.25 & 69.56 & 6.86 & 23.58 \\
\hline
\end{tabular}




\section{A.8 Impacts of Travel Time Link Length}

Table A-16 Travel Time Estimation Performances with Different Travel Time Link Lengths for Simulated Uncongested Conditions

\begin{tabular}{|c|c|c|c|c|c|c|c|}
\hline Method & Origin-Destination & $\begin{array}{c}\text { Distance } \\
\text { (Miles) }\end{array}$ & $\begin{array}{l}\text { MAE } \\
\text { (Min.) }\end{array}$ & $\begin{array}{c}\text { MAPE } \\
(\%)\end{array}$ & $\begin{array}{c}\text { Reliability } \\
\text { (\%) }\end{array}$ & $\begin{array}{c}\% \\
\text { Early }\end{array}$ & $\begin{array}{c}\% \\
\text { Late }\end{array}$ \\
\hline \multirow{4}{*}{$\begin{array}{l}\text { Point-to- } \\
\text { Point } \\
\text { Method }\end{array}$} & DS-1523E-DS-1549E & 4.24 & 0.05 & 1.17 & 100 & 0 & 0 \\
\hline & DS-1521E-DS-1549E & 4.55 & 0.05 & 1.11 & 99.64 & 0.08 & 0.28 \\
\hline & DS-1517E-DS-1549E & 5.27 & 0.07 & 1.27 & 100 & 0 & 0 \\
\hline & DS-1509E-DS-1549E & 6.42 & 0.12 & 1.92 & 100 & 0 & 0 \\
\hline \multirow{4}{*}{$\begin{array}{l}\text { Mid-Point } \\
\text { Method }\end{array}$} & DS-1523E-DS-1549E & 4.24 & 0.05 & 1.16 & 100 & 0 & 0 \\
\hline & DS-1521E-DS-1549E & 4.55 & 0.05 & 1.15 & 99.69 & 0.08 & 0.23 \\
\hline & DS-1517E-DS-1549E & 5.27 & 0.06 & 1.09 & 100 & 0 & 0 \\
\hline & DS-1509E-DS-1549E & 6.42 & 0.08 & 1.31 & 100 & 0 & 0 \\
\hline \multirow{4}{*}{$\begin{array}{l}\text { Hybrid } \\
\text { Model } 1\end{array}$} & DS-1523E-DS-1549E & 4.24 & 0.05 & 1.16 & 100 & 0 & 0 \\
\hline & DS-1521E-DS-1549E & 4.55 & 0.05 & 1.15 & 99.69 & 0.08 & 0.23 \\
\hline & DS-1517E-DS-1549E & 5.27 & 0.06 & 1.09 & 100 & 0 & 0 \\
\hline & DS-1509E-DS-1549E & 6.42 & 0.08 & 1.31 & 100 & 0 & 0 \\
\hline \multirow{4}{*}{$\begin{array}{l}\text { Hybrid } \\
\text { Model } 2\end{array}$} & DS-1523E-DS-1549E & 4.24 & 0.05 & 1.16 & 100 & 0 & 0 \\
\hline & DS-1521E-DS-1549E & 4.55 & 0.05 & 1.15 & 99.69 & 0.08 & 0.23 \\
\hline & DS-1517E-DS-1549E & 5.27 & 0.06 & 1.09 & 100 & 0 & 0 \\
\hline & DS-1509E-DS-1549E & 6.42 & 0.08 & 1.31 & 100 & 0 & 0 \\
\hline
\end{tabular}

Table A-17 Travel Time Estimation Performances with Different Travel Time Link Lengths for Simulated Incident Conditions

\begin{tabular}{|l|l|c|c|c|c|c|c|}
\hline Method & Origin-Destination & $\begin{array}{c}\text { Distance } \\
\text { (Miles) }\end{array}$ & $\begin{array}{c}\text { MAE } \\
\text { (Min.) }\end{array}$ & $\begin{array}{c}\text { MAPE } \\
\text { (\%) }\end{array}$ & $\begin{array}{c}\text { Reliability } \\
\mathbf{( \% )}\end{array}$ & $\begin{array}{c}\text { \% } \\
\text { Early }\end{array}$ & $\begin{array}{c}\text { \% } \\
\text { Late }\end{array}$ \\
\hline \multirow{3}{*}{$\begin{array}{l}\text { Point-to- } \\
\text { Point } \\
\text { Method }\end{array}$} & DS-1523E-DS-1549E & 4.24 & 0.92 & 14.00 & 86.78 & 0 & 13.22 \\
\cline { 2 - 9 } & DS-1521E-DS-1549E & 4.55 & 1.07 & 13.27 & 77.93 & 4.59 & 17.48 \\
\cline { 2 - 8 } & DS-1517E-DS-1549E & 5.27 & 1.86 & 16.59 & 57.41 & 2.49 & 40.10 \\
\cline { 2 - 9 } & DS-1509E-DS-1549E & 6.42 & 2.07 & 16.28 & 57.32 & 1.78 & 40.90 \\
\hline \multirow{3}{*}{\begin{tabular}{l} 
Mid-Point \\
\cline { 2 - 9 }
\end{tabular}} & DS-1523E-DS-1549E & 4.24 & 1.13 & 17.15 & 73.36 & 0 & 26.64 \\
\cline { 2 - 8 } & DS-1521E-DS-1549E & 4.55 & 1.44 & 17.28 & 75.94 & 0.09 & 23.97 \\
\cline { 2 - 8 } & DS-1517E-DS-1549E & 5.27 & 1.74 & 15.85 & 58.30 & 1.38 & 40.32 \\
\cline { 2 - 8 } & DS-1509E-DS-1549E & 6.42 & 1.86 & 14.78 & 59.05 & 4.19 & 36.76 \\
\hline & DS-1523E-DS-1549E & 4.24 & 0.63 & 10.10 & 91.98 & 0.35 & 7.67 \\
\hline
\end{tabular}




\begin{tabular}{|l|l|c|c|c|c|c|c|}
\hline \multirow{2}{*}{ Method } & Origin-Destination & $\begin{array}{c}\text { Distance } \\
\text { (Miles) }\end{array}$ & $\begin{array}{c}\text { MAE } \\
\text { (Min.) }\end{array}$ & $\begin{array}{c}\text { MAPE } \\
\mathbf{( \% )}\end{array}$ & $\begin{array}{c}\text { Reliability } \\
\mathbf{( \% )}\end{array}$ & $\begin{array}{c}\text { \% } \\
\text { Early }\end{array}$ & $\begin{array}{c}\% \\
\text { Late }\end{array}$ \\
\hline \multirow{3}{*}{$\begin{array}{l}\text { Hybrid } \\
\text { Model 1 }\end{array}$} & DS-1521E-DS-1549E & 4.55 & 0.85 & 11.09 & 87.02 & 0.13 & 12.85 \\
\cline { 2 - 9 } & DS-1517E-DS-1549E & 5.27 & 0.97 & 9.97 & 75.48 & 6.10 & 18.43 \\
\cline { 2 - 9 } & DS-1509E-DS-1549E & 6.42 & 1.11 & 10.24 & 70.59 & 10.34 & 19.07 \\
\hline \multirow{3}{*}{$\begin{array}{l}\text { Hybrid } \\
\text { Model 2 }\end{array}$} & DS-1523E-DS-1549E & 4.24 & 0.88 & 13.69 & 91.40 & 0 & 8.60 \\
\cline { 2 - 9 } & DS-1521E-DS-1549E & 4.55 & 1.05 & 13.50 & 81.94 & 4.59 & 13.47 \\
\cline { 2 - 9 } & DS-1517E-DS-1549E & 5.27 & 1.05 & 10.90 & 75.52 & 3.38 & 21.10 \\
\cline { 2 - 9 } & DS-1509E-DS-1549E & 6.42 & 1.15 & 10.12 & 74.50 & 3.33 & 22.17 \\
\hline
\end{tabular}

\section{A.9 Impacts of Travel Time Posting Range}

Table A-18 Travel Time Estimation Reliability with Different Posted Travel Time Ranges for Simulated Uncongested Conditions

\begin{tabular}{|c|c|c|c|c|}
\hline Method & $\begin{array}{l}\text { Range of Posted } \\
\text { Travel Time }\end{array}$ & $\begin{array}{c}\text { Reliability } \\
\text { (\%) }\end{array}$ & \% Early & \% Late \\
\hline \multirow{4}{*}{ Point-to-Point Method } & {$[\mathrm{TT}-2, \mathrm{TT}+2]$} & 100 & 0 & 0 \\
\hline & {$[\mathrm{TT}-1, \mathrm{TT}+2]$} & 100 & 0 & 0 \\
\hline & {$[\mathrm{TT}-1, \mathrm{TT}+1]$} & 99.29 & 0 & 0.71 \\
\hline & [TT-0.5, TT+0.5] & 70.07 & 0.42 & 29.52 \\
\hline \multirow{4}{*}{ Mid-Point Method } & {$[\mathrm{TT}-2, \mathrm{TT}+2]$} & 100 & 0 & 0 \\
\hline & {$[\mathrm{TT}-1, \mathrm{TT}+2]$} & 100 & 0 & 0 \\
\hline & {$[\mathrm{TT}-1, \mathrm{TT}+1]$} & 99.29 & 0 & 0.71 \\
\hline & [TT-0.5, TT+0.5] & 70.07 & 0.42 & 29.52 \\
\hline \multirow{4}{*}{ Hybrid Model 1} & [TT-2, TT+2] & 100 & 0 & 0 \\
\hline & {$[\mathrm{TT}-1, \mathrm{TT}+2]$} & 100 & 0 & 0 \\
\hline & {$[\mathrm{TT}-1, \mathrm{TT}+1]$} & 99.29 & 0 & 0.71 \\
\hline & [TT-0.5, TT+0.5] & 70.07 & 0.42 & 29.52 \\
\hline \multirow{4}{*}{ Hybrid Model 2} & [TT-2, TT+2] & 100 & 0 & 0 \\
\hline & [TT-1, TT+2] & 100 & 0 & 0 \\
\hline & [TT-1, TT+1] & 99.29 & 0 & 0.71 \\
\hline & [TT-0.5, TT+0.5] & 70.07 & 0.42 & 29.52 \\
\hline
\end{tabular}


Table A-19 Travel Time Estimation Reliability with Different Posted Travel Time Ranges for Simulated Incident Conditions

\begin{tabular}{|c|c|c|c|c|}
\hline Method & $\begin{array}{l}\text { Range of Posted } \\
\text { Travel Time }\end{array}$ & $\begin{array}{c}\text { Reliability } \\
\text { (\%) }\end{array}$ & \% Early & \% Late \\
\hline \multirow{6}{*}{ Point-to-Point Method } & [TT-2, TT+3] & 57.32 & 1.78 & 40.90 \\
\hline & [TT-2, TT+4] & 58.93 & 1.78 & 39.29 \\
\hline & [TT-2, TT+5] & 59.97 & 1.78 & 38.25 \\
\hline & [TT-1, TT+4] & 56.58 & 4.14 & 39.29 \\
\hline & [TT-1, TT+5] & 57.61 & 4.14 & 38.25 \\
\hline & [TT-1, TT+6] & 58.42 & 4.14 & 37.45 \\
\hline \multirow{6}{*}{ Mid-Point Method } & [TT-2, TT+3] & 59.05 & 4.19 & 36.76 \\
\hline & {$[\mathrm{TT}-2, \mathrm{TT}+4]$} & 64.85 & 4.19 & 30.96 \\
\hline & [TT-2, TT+5] & 68.18 & 4.19 & 27.63 \\
\hline & [TT-1, TT+4] & 62.44 & 6.61 & 30.96 \\
\hline & [TT-1, TT+5] & 65.77 & 6.61 & 27.63 \\
\hline & [TT-1, TT+6] & 67.57 & 6.61 & 25.85 \\
\hline \multirow{6}{*}{ Hybrid Model 1} & {$[\mathrm{TT}-2, \mathrm{TT}+3]$} & 70.59 & 10.34 & 19.07 \\
\hline & [TT-2, TT+4] & 73.12 & 10.34 & 16.54 \\
\hline & [TT-2, TT+5] & 75.13 & 10.34 & 14.53 \\
\hline & [TT-1, TT+4] & 65.02 & 18.44 & 16.54 \\
\hline & [TT-1, TT+5] & 67.03 & 18.44 & 14.53 \\
\hline & [TT-1, TT+6] & 68.12 & 18.44 & 13.44 \\
\hline \multirow{6}{*}{ Hybrid Model 2} & [TT-2, TT+3] & 74.50 & 3.33 & 22.17 \\
\hline & [TT-2, TT+4] & 75.88 & 3.33 & 20.79 \\
\hline & [TT-2, TT+5] & 75.88 & 3.33 & 20.79 \\
\hline & [TT-1, TT+4] & 69.67 & 9.54 & 20.79 \\
\hline & [TT-1, TT+5] & 69.67 & 9.54 & 20.79 \\
\hline & {$[\mathrm{TT}-1, \mathrm{TT}+6]$} & 70.25 & 9.54 & 20.22 \\
\hline
\end{tabular}


VITA

YAN XIAO

EDUCATION

1993 - $1997 \quad$ B.S., Thermal Engineering

Chongqing University, Chongqing, China

1997 - $2000 \quad$ M.S., Thermal Engineering

Chongqing University, Chongqing, China

2003 - 2006 M.S., Mechanical Engineering

University of Connecticut, Storrs, Connecticut

2006 - 2011 Doctoral Candidate in Civil Engineering

Florida International University, Miami, Florida

EMPLOYMENT

$2000-2003 \quad$ Teacher

Fujian Normal University, Fuzhou, China

2003-2004 Graduate Teaching Assistant

Department of Mechanical Engineering

University of Connecticut, Storrs, Connecticut

2004-2006 Graduate Research Assistant

Department of Mechanical Engineering

University of Connecticut, Storrs, Connecticut

2006- 2010 Graduate Research Assistant

Lehman Center for Transportation Research

Florida International University, Miami, Florida

\section{AFFILIATIONS}

- $\quad$ Student member, Women in Transportation, 2011 - Present

- $\quad$ Student member, Chi Epsilon Honor Society, 2008 - Present

- $\quad$ Student member, Institute of Transportation Engineers, 2006 - Present 


\section{PUBLICATIONS AND PRESENTATIONS}

- Zhan, C., Alvarez, P., Hadi, M., and Xiao, Y. (2011). "Software-in-the-Loop Simulation in Support of Evaluating Traffic Management Center Software." Presented at the 90th Annual Meeting of the Transportation Research Board, Washington, DC.

- $\quad$ Xiao, Y., Hadi, M., Ozen, H., and Mysore, V. (2010). "An ITS Evaluation Tool in the FSUTMS Regional Demand Modeling Environment.” Transportation Research Record: Journal of the Transportation Research Board, No. 2176, pp. 76-83.

- $\quad$ Hadi, M., Xiao, Y., Zhan, C. and Gan, A. (2010). "Assess the Benefits of Incident Management Systems: Combining Freeway Facility Procedures from the Highway Capacity Manual and Data Archives of Intelligent Transportation Systems.” Transportation Research Record: Journal of the Transportation Research Board, No. 2173, pp. 115-122.

- $\quad$ Qiang, H., Hadi, M., Xiao, Y., and Zhan, C. (2010). "Estimating Incident Detection Time Based on Incident Management and Traffic Detector Data.” Presented at the 89th Annual Meeting of the Transportation Research Board, Washington, DC.

- $\quad$ Hadi, M., Shen, L., Li, J., and Xiao, Y. (2010). "Simulation Tool to Assess Impacts of Parameters of Service Patrol Operations." Transportation Research Record: Journal of the Transportation Research Board, No. 2178, pp. 111-118.

- Xiao, Y., and Hadi, M. (2008). "Integrated Intelligent Transportation System Evaluation and Demand Forecasting Environment.” Presented at the 87th Annual Meeting of the Transportation Research Board, Washington, DC.

- $\quad$ Hadi, M., Shen, L., Zhan, C., Xiao, Y., Corbin, S., and Chen, D. (2008). "Operation Data for Evaluating Benefits and Costs of Advanced Traffic Management Components." Transportation Research Record: Journal of the Transportation Research Board, No. 2086, pp. 48-55.

\section{HONORS AND AWARDS}

- $\quad$ Dissertation Year Fellowship, Florida International University, 2010-2011.

- District 10 ITE Best Student Chapter Award, while serving as the Chapter Vice President, 2010

- $\quad$ AASHTO Franccis B. Francois Award, 2009

- $\quad$ Florida ITE Bill McGrath Scholarship Award, 2009

- $\quad$ Best Student Paper Award, District 10 ITE, 2009

- Winner of Citilabs' 2008 Summer of Cube Student Research Competition, 2008

- $\quad$ ITS Florida Anne S. Brewer Scholarship Award, 2007

- International ITE Best Student Chapter Award, while serving as the Chapter's Treasurer, 2007 